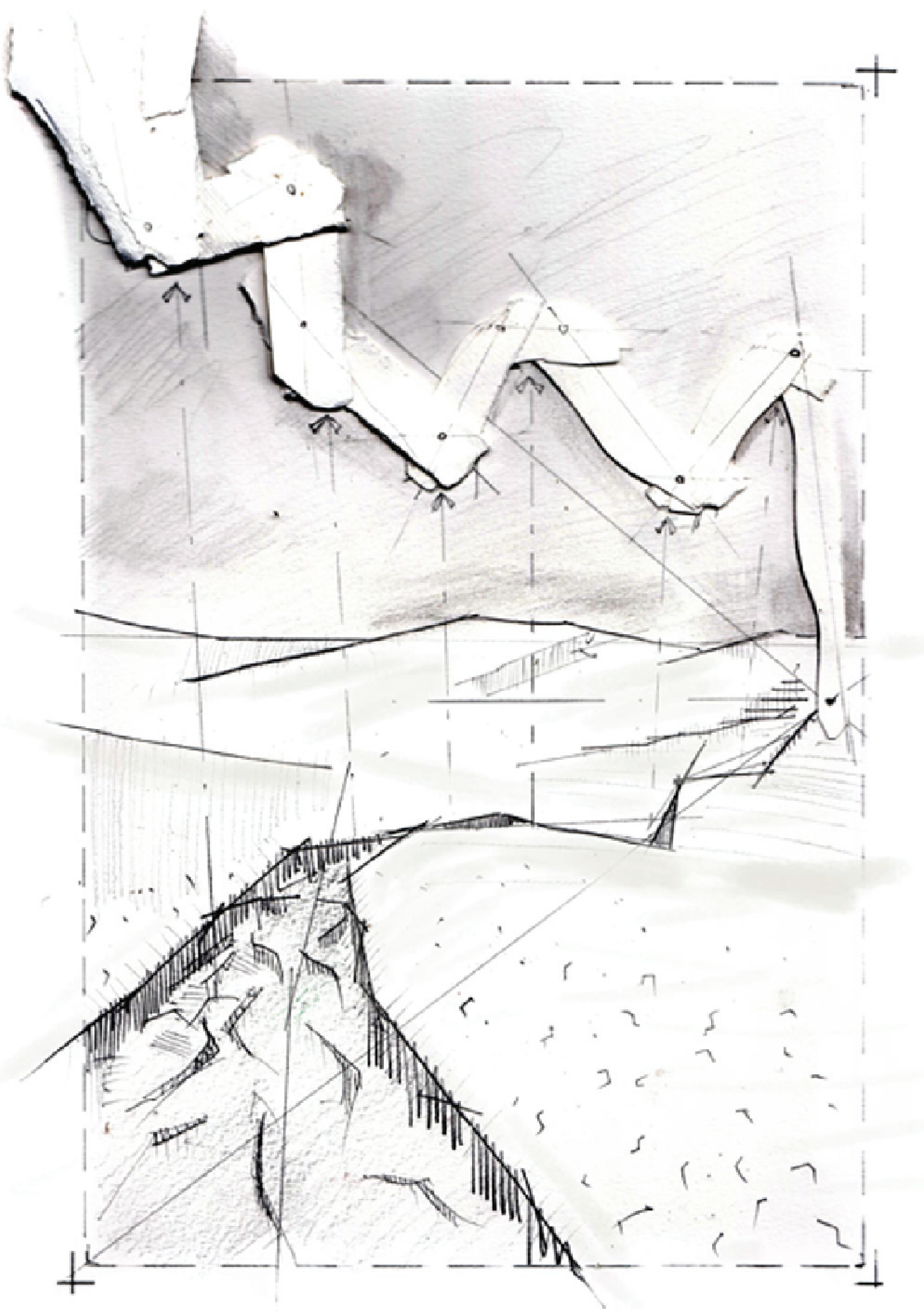

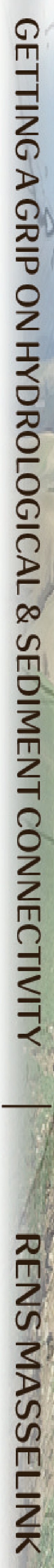

GETTING A GRIP ON HYDROLOGICAL \& SEDIMENT CONNECTIVITY

\section{RENS MASSELINK}

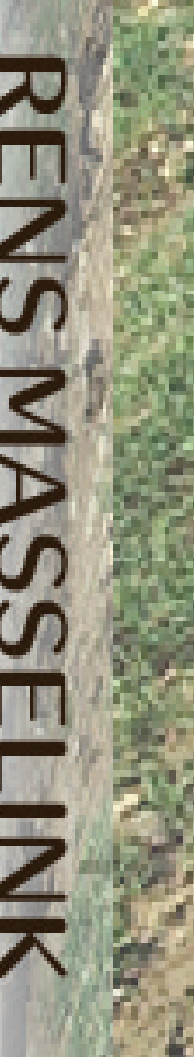




\section{Getting A Grip on Hydrological and Sediment Connectivity}

Rens J.H. Masselink 


\section{Thesis committee}

\section{Promotors}

Prof. Dr C.J. Ritsema

Professor Soil Physics and Land Management

Wageningen University \& Research

Prof. Dr S.E.A.T.M. van der Zee

Personal chair, Soil Physics and Land Management Group

Wageningen University \& Research

\section{Co-promotors}

Dr S.D. Keesstra

Associate Professor, Soil Physics and Land Management Group

Wageningen University \& Research

Dr A.J.A.M. Temme

Assistant Professor, Soil Geography and Landscape Group

Wageningen University \& Research

Assistant professor, Geography department, University of Kansas, USA

\section{Other members}

Prof. Dr A.K. Bregt, Wageningen University \& Research

Prof. J.S. Rowan, University of Dundee, United Kingdom

Dr L. Wittenberg, University of Haifa, Israel

Dr D. Vericat, University of Lleida, Spain

This research was conducted under the auspices of the Graduate School for SocioEconomic and Natural Sciences of the Environment (SENSE) 


\title{
Getting A Grip on Hydrological and Sediment Connectivity
}

\author{
Rens J.H. Masselink
}

Thesis

submitted in fulfilment of the requirements for the degree of doctor at Wageningen University by the authority of the Rector Magnificus,

Prof. Dr A.P.J. Mol

in the presence of the

Thesis Committee appointed by the Academic Board

to be defended in public

on Thursday 7 September 2017

at 11 a.m. in the Aula. 
Rens J.H. Masselink

Getting a grip on hydrological and sediment connectivity 170 pages

PhD thesis, Wageningen University, Wageningen, NL (2017)

With references, with summary in English, Dutch and Spanish

ISBN 978-94-6343-634-2

DOI $10.18174 / 419764$ 
"Everything is connected to everything else, but near things are stronger connected than distant things"

Adapted First Law of Geography by Waldo Tobler (1970) 



\section{Table of Contents}

Chapter 1. General Introduction

Chapter 2. A network theory approach for a better understanding of overland flow connectivity

Chapter 3. Assessing hillslope-channel connectivity in an agricultural catchment using rareearth oxide tracers and random forest models

Chapter 4. Modelling discharge and sediment yield at catchment scale using connectivity components.

Chapter 5. Scale matters: contrasting hillslope and catchment scale calibration results for hydrology and sediment fluxes using the LAPSUS-D model.

Chapter 6. Synthesis.

Appendix A

Appendix B

Appendix C.

Bibliography 123

Summary. 139

Samenvatting. 143

Resumen.

Dankwoord / Agradecimientos / Acknowledgements

Publications. 153

About the author. 155 



\section{Chapter 1}

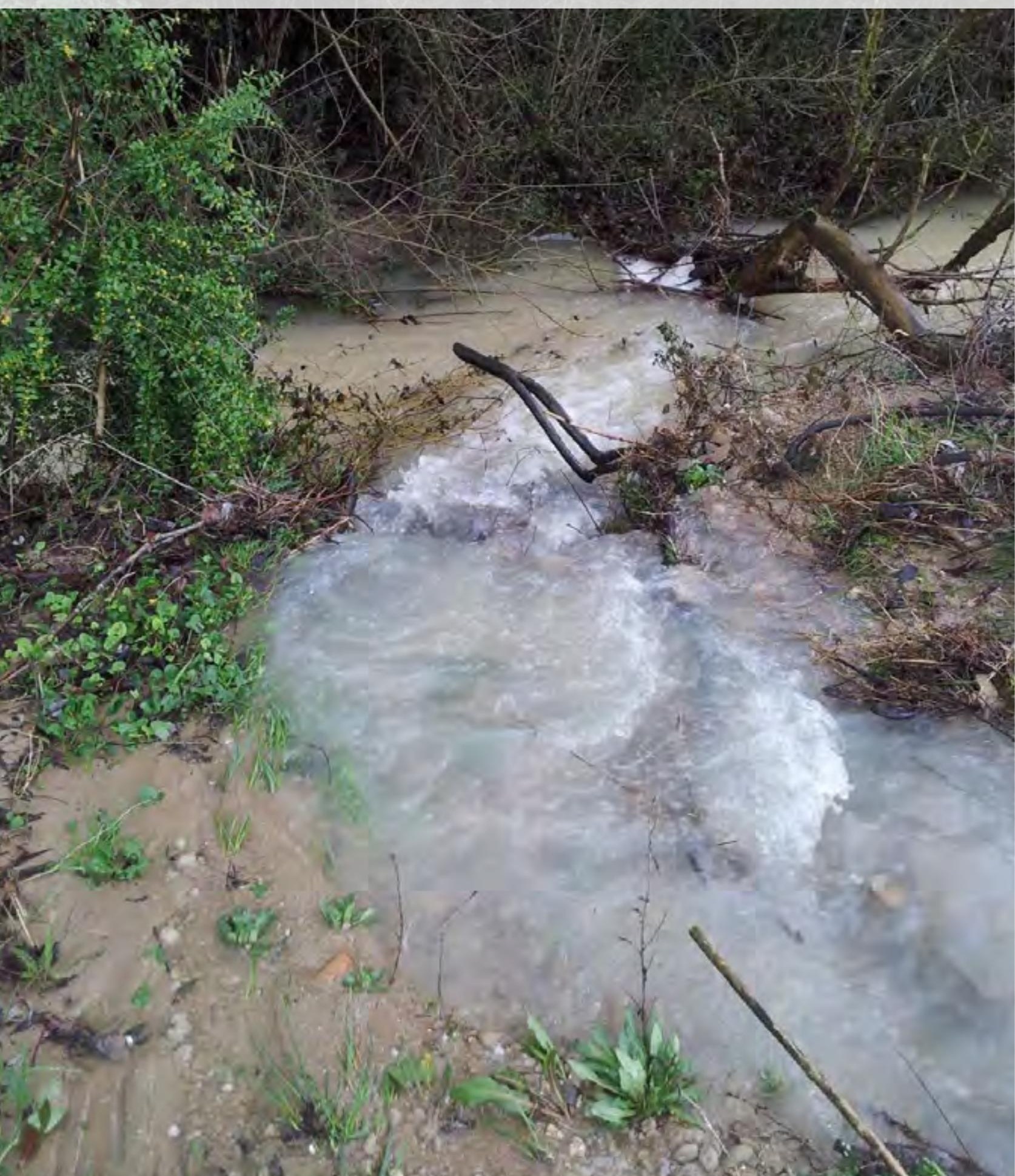





\section{Chapter 1. General Introduction}

\subsection{Negative Effects of Soil Erosion}

Land degradation is a large problem worldwide, especially in agricultural areas. Between 1-6 billion ha of land worldwide is affected by land degradation (Gibbs and Salmon, 2015). With an increasing world population, more food production is needed and, therefore, more land is converted into agricultural areas (Schmitz et al., 2014). This conversion of land to agricultural areas, in turn, leads to more land degradation. Some common forms of land degradation are desertification, salinization and soil erosion by water.

Soil erosion by water has negative on-site and off-site effects. On-site effects occur at the location where erosion takes place. These negative effects include the loss of nutrients and fertile topsoil which result in productivity loss. Off-site effects occur because of downstream sediment and/or nutrients transport. Off-site effects of soil erosion by water include sedimentation and eutrophication of waterways and reservoirs (Morgan, 2009; Mekonnen et al., 2015a).

The negative effects of soil erosion have been acknowledged for a long time. Since the beginning of the $20^{\text {th }}$ century, researchers have tried to quantify soil displaced due to water, and to measure and model the efficiency of management strategies.

\subsection{Measuring and Modelling Soil Erosion}

The traditional approach in assessing soil erosion problems mainly involves measuring plot scale runoff and erosion. Processes that influence soil erosion have been measured and described physically. These measurements were generally carried out in laboratory or small plot scale settings. Infiltration rates are measured using rainfall simulators that simulate rainfall on a small plot $\left(\sim 0.5-2 \mathrm{~m}^{2}\right)$ or with measurements of saturated hydraulic conductivity, often done in the lab (Iserloh et al., 2013; Keesstra et al., 2014a; Cerdà et al., 2016). It has been generally recognised that these characteristics of the soil and vegetation are highly heterogeneous and it is, therefore, difficult to obtain representative measurements for large areas. An example of heterogeneity in soils and vegetation is reinfiltration of overland flow at lower positions on the hillslope due to macropores or other preferential flow paths. For vegetation, different vegetation patterns might cause different behaviour in terms of runoff and sediments (Keesstra et al., 2009b; Moreno-de las Heras et al., 2012; Poeppl et al., 2012; Okin et al., 2015).

Other small scale processes like splash erosion and sheet erosion have been studied in detail to extract physical relations between factors such as drop size, kinetic energy, soil texture and splash erosion (Ellison, 1944; Park et al., 1982). However, all these relations that are extracted in the lab or at the plot scale are not necessarily valid anymore when used at catchment scale (Boix-Fayos et al., 2006; Cerdan et al., 2010). These processes might instead, show highly non-linear responses, making it difficult to extrapolate them to larger areas (i.e. from plot scale to catchment scale). Furthermore, it was demonstrated that at different scales, different processes become the dominant 
determinant for the amount of sediment transport (de Vente and Poesen, 2005; de Vente et al., 2007).

Physically-based erosion models also use make use of processes and variables measured at small-scales to predict erosion and deposition within entire catchments. These models are subsequently used for prediction of effects of management practices (de Roo et al., 1996; López-Vicente et al., 2013a). Many of these models rely heavily on (subjective) calibration of the parameters measured at small scale to produce good modelling results at larger scale (Kalantari et al., 2015). This calibration procedure often leads to unrepresentative values for model parameters, which could lead to wrong process representation. Furthermore, the calibration is often done with data at the outlet only, meaning there is no control over whether processes within the catchment are adequately modelled. Moreover, calibrating a model with multiple parameters can lead to the problem of equifinality. Equifinality for modelling means that many model structures or parameter sets within a given model framework will predict a required output (Beven, 1996; Brazier et al., 2000). This means that multiple possibilities exist to obtain a correctly modelled value as an output, but that not all (or even none) of these possibilities are actually correct.

Problems with upscaling, misrepresentation of processes valid at plot and lab scale, and equifinality are issues that make it difficult to properly predict sediment sources, pathways and sinks within catchments. In order to deal with these non-linear processes at large spatial scales and related lack of proper representation of water and sediment sources, pathways and sinks, a new concept was developed.

\subsection{The Connectivity Concept}

Recently, the connectivity concept has emerged within geomorphology and hydrology in order to cope with non-linear nature of processes of water and sediment transport. There has been a shift from thinking about sediment transfer between different compartments to a continuum-based approach trying to understand pathways, routes and scales of movement of sediment (Bracken et al., 2015). Connectivity is well integrated within several other disciplines for many years, for example, ecology (Ward, 1989; Poole, 2002), social network science (Hummon and Dereian, 1989) and neuroscience (Leuchter et al., 1992). Many of the concepts and techniques used in these disciplines can aid in advancing the understanding of hydrological and sediment connectivity.

The importance of connectivity for runoff and sediment transport was already mentioned in 2000 (Bull et al., 2000). But even before that, researchers acknowledged the importance of the strength of connections between the different landscape compartments, e.g. hillslopes and channels. All these previous studies used varying concepts and definitions. A few of the terms and definitions closely related to connectivity are hillslope-channel coupling, variable contributing areas, or many of the similar terms of hydrologically similar response areas/units (Beven and Kirkby, 1979; Michaelides and Wainwright, 2002; Ambroise, 2004; Buchanan et al., 2012). One commonly used concept is the sediment delivery ratio: the amount of sediments leaving 
the catchment to the gross amount of sediments eroded within the catchment (Glymph, 1954; Maner, 1958; Roehl, 1962). The sediment delivery ratio is influenced by a wide range of factors as topography, vegetation and soils. However, no meaningful relationship between any of these factors and the sediment delivery ratio can be found that works for all environments. The sediment delivery ratio is, furthermore, very dependent on catchment size, which makes it unusable for comparing catchments of varying sizes. Additionally, the sediment delivery ratio is not informative for the location of sources of sediments that reach the outlet of the catchment (Walling, 1983).

\subsection{1 (Too) many Concepts of Connectivity}

Over the past years, multiple conceptual frameworks have been developed in parallel in many different research groups around the world, who all used different terminology and ideas about connectivity (Table 1.1). To deal with this, an EU funded COST action ("ConnectEur: Connecting European Connectivity Research") was set up to bring scientists from all over Europe together to discuss the topic of connectivity and to advance and streamline the ideas and concepts. To date, however, no unified theory and measuring and modelling approaches have been published as a result of these efforts. However, most definitions are close to the following definition of hydrological connectivity by (Pringle, 2003): 'Hydrologic connectivity is the water-mediated transport of matter, energy and organisms within or between elements of the hydrologic cycle' (Table 1.1).

The majority of the numerous studies on hydrological and sediment connectivity over the past decade have focussed on conceptual frameworks, indices and modelling. The conceptual frameworks and models describe the components of catchment connectivity (Bracken and Croke, 2007; Turnbull et al., 2008; Lexartza-Artza and Wainwright, 2009; Fryirs, 2012; Bracken et al., 2013, 2015). Indices and metrics used to describe connectivity were based on topography using Digital Elevation Models (DEMs) (Cavalli et al., 2013), soil moisture (Ali and Roy, 2010) or a combination of DEM based and field based studies (Borselli et al., 2008).

There are several possibilities to describe connectivity: as a nominal variable (Fryirs, 2012), as a scalar (breakthrough volume; (Hairsine et al., 2002; Croke and Hairsine, 2006) or as complex vectors (Magnitude-frequency distribution; (Bracken et al., 2015). The problem, however, with these types of descriptions for connectivity is that they need to be measured/assessed at a single scale level and are then not always suitable to be applied over a whole catchment.

In the fields of ecology and neuroscience, connectivity is divided into structural and functional connectivity (Stubbs, 1978; Selman and Doar, 1992). Many of the early conceptual frameworks on connectivity also employed this separation (Bracken and Croke, 2007; Turnbull et al., 2008; Wainwright et al., 2011). Structural connectivity is the form and architecture of the landscape, while all the processes acting upon that structure are captured within functional connectivity (later referred to as processbased connectivity by (Bracken et al., 2013). These definitions are analogous to geomorphology in general, where a distinction is made between landforms and the 
processes that form and act upon these landforms. Within connectivity, however, the distinction between structural and functional connectivity is dependent on the spatial and temporal extent of the study (ie. when does structural connectivity become functional?). Continuous feedbacks between the processes that act upon the structure and the structure affecting the processes, and the co-evolution of vegetation, soils and topography further impede making a clear distinction between the two. For the past decade, researchers have struggled to measure and model functional and structural connectivity, while making use of one of the many conceptual frameworks (Table 1.1).

\subsubsection{Measuring and Modelling Connectivity}

Although the number of studies on measuring connectivity has been slowly increasing, there are still relatively few studies with the objective of measuring connectivity. Instead, connectivity is used to explain, for example, variability in the measured data (Cammeraat, 2002). Some examples of studies in which the main objective was to infer connectivity from measurements are James and Roulet (2007), who measured antecedent moisture conditions to find a highly non-linear response for different rainfall events, and Ali and Roy (2010), who used a network of soil moisture sensors to assess 2D and 3D connectivity patterns in soil moisture and assessed how these patterns influence runoff.

Currently, the consensus among the hydrological and geomorphological research community is that connectivity as such, cannot be measured directly, but has to be inferred from measurements of other variables. Structural connectivity can be inferred from measurements of topography and landscape structure, while functional connectivity needs to be inferred from measurements of fluxes and sediment tracing (Brazier et al., In prep.).

The techniques to measure landscape structure, water and sediment fluxes and sediment tracing have improved considerably over the past couple of years. Using Unmanned Aerial Vehicles (UAVs) and Structure-from-Motion photogrammetry it is now possible to obtain DEMs of large areas with centimetre resolution at high accuracy (Westoby et al., 2012). The amount of techniques available for sediment tracing have increased substantially as well over the past decades (Guzmán et al., 2013). These techniques, however, have not been used to their full potential for connectivity studies, while especially the combination of techniques is potentially powerful.

Similarly, modelling studies on connectivity, have been mainly conceptual, empirical or exploratory and most did not use real world data for validation (Meerkerk et al., 2009; Gumiere et al., 2011; Baartman et al., 2013; Kirkby, 2014). On the one hand, connectivity has been explicitly applied as an input variable to models: as a connectivity function for describing sub-grid surface roughness (Antoine et al., 2009; Peñuela et al., 2012), or by using a connectivity index as model input (Cavalli et al., 2013). On the other hand, connectivity on hillslopes and catchments has been an output of models using changing (artificial) topography (Baartman et al., 2013) or differences in vegetation (Coulthard and Van De Wiel, 2016). The lack of modelling studies on connectivity using 
real-world data shows the need for measured data from a variety of sources from which connectivity can be inferred.

Table 1.1 Definitions of connectivity, grouped by the support level at which they are defined, adapted from (Ali and Roy, 2009).

Definition

Reference

Landscape Compartments

How variable source areas and channels are connected to each other, and can be Kirkby et al., 2002 considered at a variety of scales

Flows of matter and energy (water, nutrients, sediments, heat, etc.) between Tetzlaff et al., 2007 different landscape components

The physical coupling between discrete units of the landscape, notably, upland and Stieglitz et al., 2003 riparian zones, and its implication for runoff generation and chemical transport (Stieglitz et al., 2003)

Spatially connected features which concentrate flow and reduce travel times.

The condition by which disparate regions on a hillslope are linked via lateral subsurface water flow.

Knudby and Carrera, 2005

Hornberger et al., 1994; Creed and Band, 1998

Connection, via the subsurface flow system, between the riparian (near stream) Vidon and Hill, 2004; zone and the upland zone (also known as the hillslope) occurs when the water table at the upland-riparian zone interface is above the confining layer.

The internal linkages between runoff and sediment generation in upper parts of catchments and the receiving waters [...] two types of connectivity: direct connectivity via new channels or gullies, and diffuse connectivity as surface runoff reaches the stream network via overland flow pathways.

An ecological context to refer to water-mediated transfer of matter, energy and/or organisms within or between elements of the hydrologic cycle.

Channel

The physical linkage of sediment through the channel system, which is the transfer of sediment from one zone or location to another and the potential for a specific particle to move through the system. (Hooke, 2003)

System as a whole

Hydrologically relevant spatial patterns of properties (e.g. high permeability) or state variables (e.g. soil moisture) that facilitate flow and transport in a hydrologic system (e.g. an aquifer or watershed)

The extent to which water and matter that move across the catchment can be stored within or exported out of the catchment

All the former and subsequent positions and times, associated with the movement of water or sediment passing through a point in the landscape

Hooke, 2003

Western et al., 2001

Lane et al., 2004

Bracken and Croke, 2007 
One common tool for the assessment of connectivity within other disciplines are networks (graphs) of the system. For neuroscience, the nodes or vertices within these graphs can represent individual neurons, or more commonly, groups of neurons called "cytoarchitectonic areas", because of the large number of neurons (Zilles et al., 2002). The connections between these (groups of) neurons form the edges within the network. Within social network science the nodes are (groups of) people and the edges are the connections between these people (Figure 1.1). These disciplines relatively easily distinguish between structural and functional connectivity of the system, and use networks to study complex interactions that are otherwise difficult to discern (van Wijk et al., 2010; Kininmonth et al., 2015).

Networks have been used in the past in geomorphological studies, mainly those looking at river systems (Strahler, 1957; Shreve, 1974; Marra et al., 2014). Within the hydrological and sediment connectivity community, however, the use of networks has been done only in a single modelling study (Heckmann and Schwanghart, 2013).

The difficulty of separating structure from function for hydrology and geomorphology makes it harder to distinguish meaningful nodes and edges for hydrological and sediment connectivity. Furthermore, whereas fluxes within ecological systems and social network sciences can go in any direction, water and sediment tend to follow the slope of a system due to gravity. This means that networks will be directed, which might result in a lower utility for the use of networks in hydrology and sediment transport dynamics.

\subsection{Objectives and research questions}

A unified theory on what constitutes connectivity and how connectivity should be measured or inferred remains one of the biggest challenges within catchment science. In addition, it is unclear whether connectivity should be an output or an input of a model, either explicitly or implicitly. The main objective of this thesis was, therefore, to assess and quantify hydrological and sediment connectivity in a meaningful way, which can advance our understanding of hydrological and sediment transport processes and catchment system dynamics.
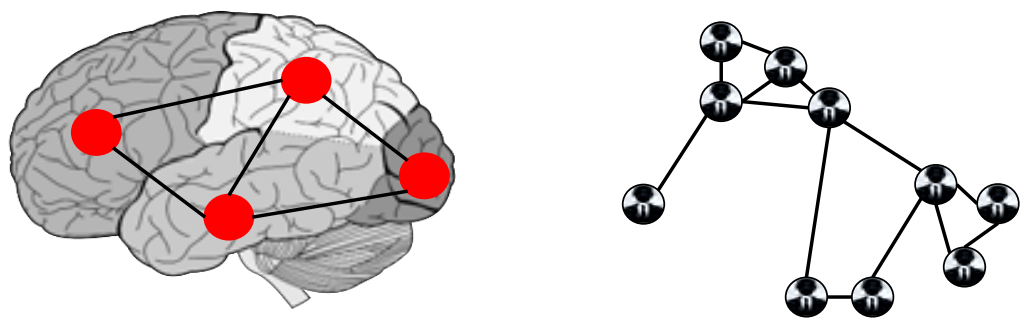

Figure 1.1 Simplified examples of networks for neuroscience (left) and social network science (right). 
With this better understanding of the functioning of the system we will be better able to apply effective management strategies at appropriate locations for the reduction of sediments and nutrients leaving agricultural fields and catchments. Several research questions were set to reach this objective:

1. Can networks aid in obtaining a better understanding of hydrological connectivity at the hillslope scale?

2. How are event characteristics linked to hillslope-channel connectivity in terms of water and sediments?

3. How are water and sediment discharge of a catchment influenced by changes in connectivity within the catchment, both spatially and temporally?

4. How do measured data at multiple temporal and spatial scales improve our understanding of hydrological and sediment connectivity and can these data help to improve hydrological and sediment transport models?

\subsection{Outline of the thesis}

The main objective and the research questions are addressed in chapters 2-6 (Figure 1.2). In chapter 2 , measured high-temporal resolution overland-flow data and highspatial resolution elevation data are analysed and linked together by using networks. From these data analyses, the differences in overland flow generation and persistence on a single hillslope was assessed and related to antecedent conditions and event characteristics. Furthermore, the results obtained at the hillslope scale were compared to data obtained at the catchment scale. This chapter helps to answer the first, and part of the second research question. Chapter 3 creates a link between measuring sediment transport data, the assessment of hillslope-channel connectivity and measured sediment discharge data at the catchment scale. Furthermore, factors that influence the differences and thresholds in connectivity are assessed using a machine learning ensemble method called Random Forest. The results from this chapter answer the second research question.

In the chapter 4 , I look at catchment scale water and sediment discharge and try to assess how temporal changes in connectivity play a role in the amount of water and sediments reaching the outlet of the channel, which links to research question 3 . In chapter 5 I use a spatially-distributed, reduced-complexity sediment transport model, calibrated at different spatial scales to determine how models can be improved to better inform managers on spatial patterns of erosion and deposition and connectivity. This relates to the answering of research questions 3 and 4. Chapter 6 is the synthesis of this thesis, which describes the main findings of the individual chapters and their implications. Furthermore, a general outlook on the way forward regarding connectivity research is given. 


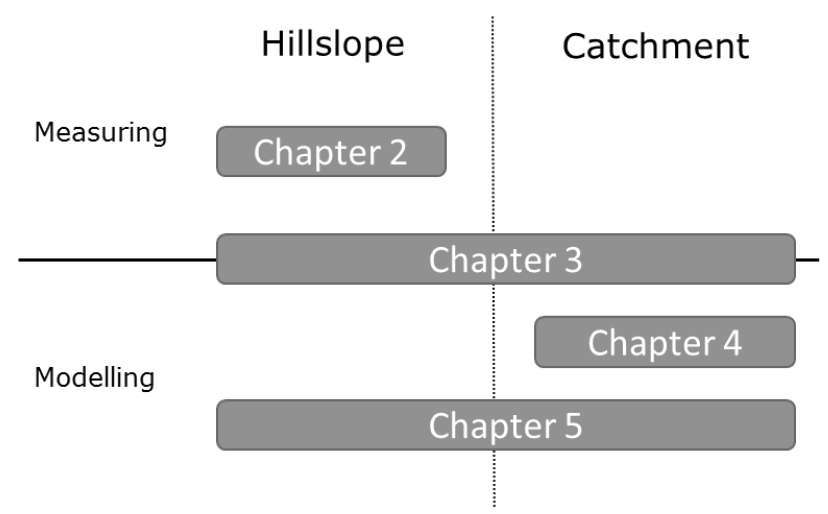

Figure 1.2 The outline, spatial scales and method of study for each of the chapters in this thesis.

\subsection{Study Area}

The study was carried out in three experimental catchments in the autonomous region of Navarre, Northern Spain (Figure 1.3). The reason why these three catchments were chosen was because of data availability and noticeable differences between the three catchments which made it possible to assess different environments. The Latxaga catchment was the studied in all four chapters, while the 'La Tejeria' catchment and the 'Oskotz' catchment were only studied in chapter 4 . The catchments were set up as research catchments by the government of Navarre to assess erosion in the province within agricultural and semi-natural catchments (Casalí et al., 2010). The catchments are part of a larger network of experimental catchments within the autonomous region of Navarre.

The Latxaga $\left(2.07 \mathrm{~km}^{2}\right)$ and La Tejeria $\left(1.69 \mathrm{~km}^{2}\right)$ catchments are agricultural catchments, that are entirely composed of agricultural fields, apart from some shrub areas which are located where agricultural practices are impossible due to too steep slopes or bedrock close to the surface. The majority of crops are winter wheat (Triticum aestivum), barley (Hordeum vulgare) and occasionally legumes (Vicia faba L. and Pisum sativum L.) or sunflower (Helianthus annuus L.). The Oskotz forest $\left(5.05 \mathrm{~km}^{2}\right)$ catchment is larger than the two agricultural catchments, and was chosen in order to model the connectivity in catchments with different size and land use. The land cover in Oskotz is mainly forest (89\%), however, some agricultural fields are present (11\%). Climate in the two agricultural catchments is similar with $750-850 \mathrm{~mm}$ of precipitation per year (Gobierno de Navarra, 2001), most of which falls in winter, although some convective storms occur during summer. The precipitation pattern is similar in Oskotz, but due to its location closer to the coast and the Pyrenees, precipitation averages to $1242 \mathrm{~mm}$ per year (Gobierno de Navarra, 2001). 
Latxaga and La Tejeria are mainly underlain by marls and within La Tejeria some sandstone is also present. The geology in Oskotz is characterised by an alternation of marls and sandy limestone (Gobierno de Navarra, 1997; Casalí et al., 2010). Soil depths in Latxaga average to $1.0 \mathrm{~m}$ and in La Tejeria to $0.8 \mathrm{~m}$. Organic matter content in both agricultural catchments is around 1-2.5\% (Casalí et al., 2008). Soils in Oskotz are deeper than 1 meter, except for those on eroded hillslopes, which are about 0.5-1 m deep. Organic matter content in Oskotz ranges between 3-6\% (Casalí et al., 2010).
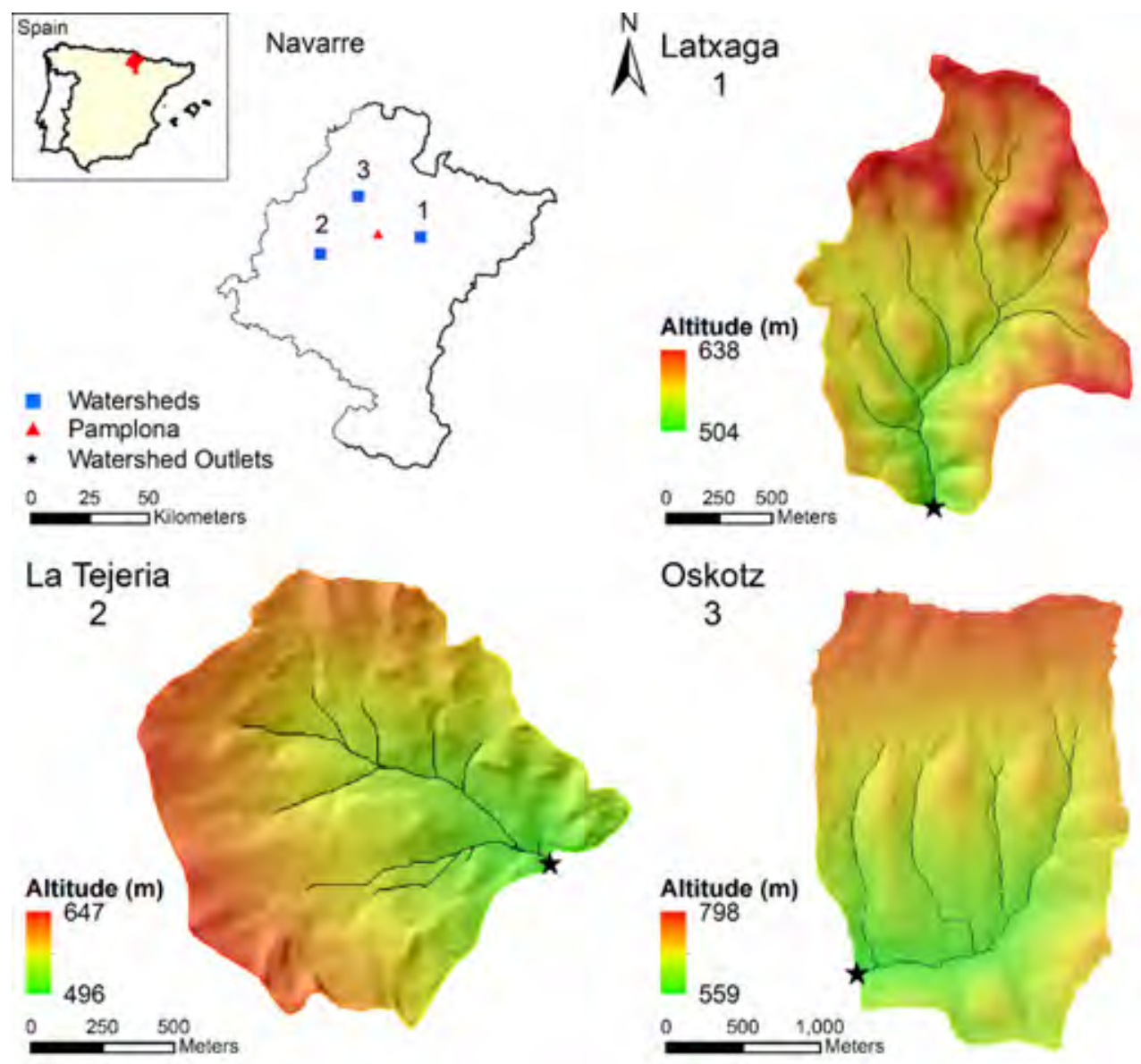

Figure 1.3 The three study areas in Navarre, Spain. 


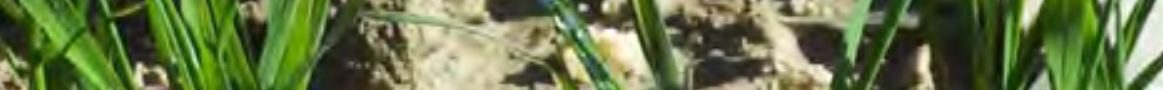

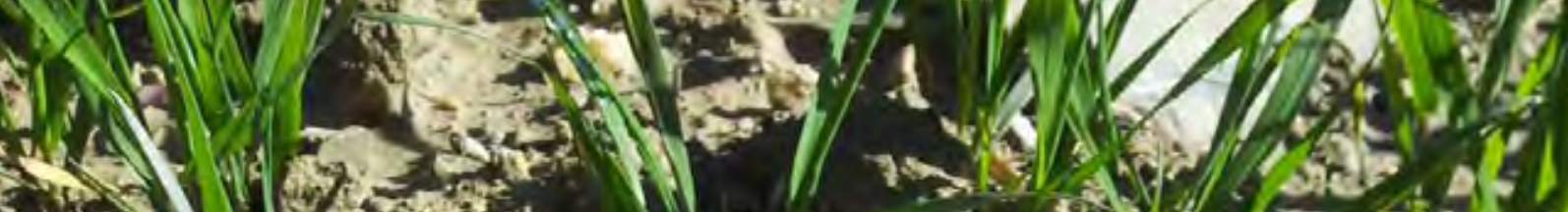

\section{Chapter 2}

$2 \times 3 \sqrt{15}$

(1)

C.1.

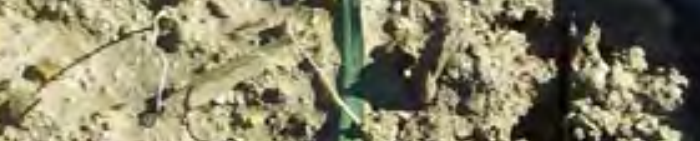

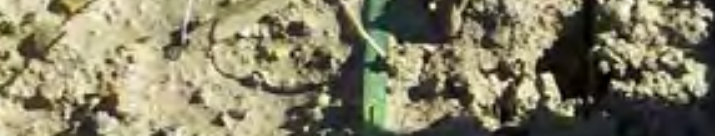
$4 x^{2} x^{2}+4$

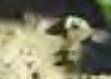

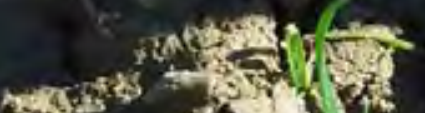
$x^{2}+y^{2} x^{2}$ WNO

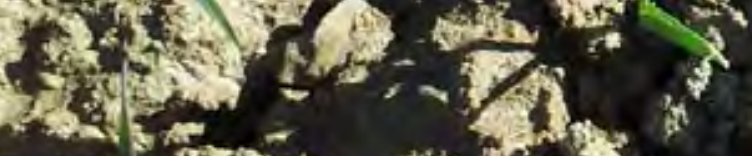

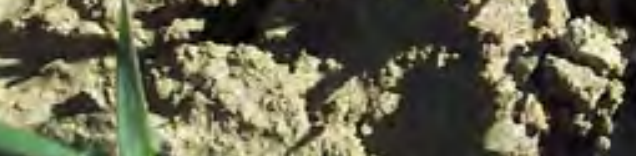
102,5 is

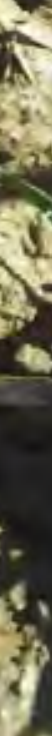

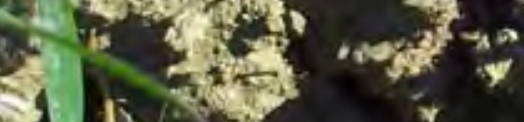
14

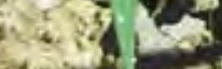

a.

(1)

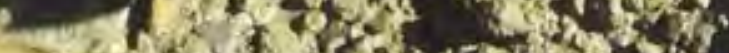
ats

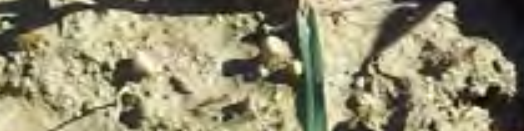
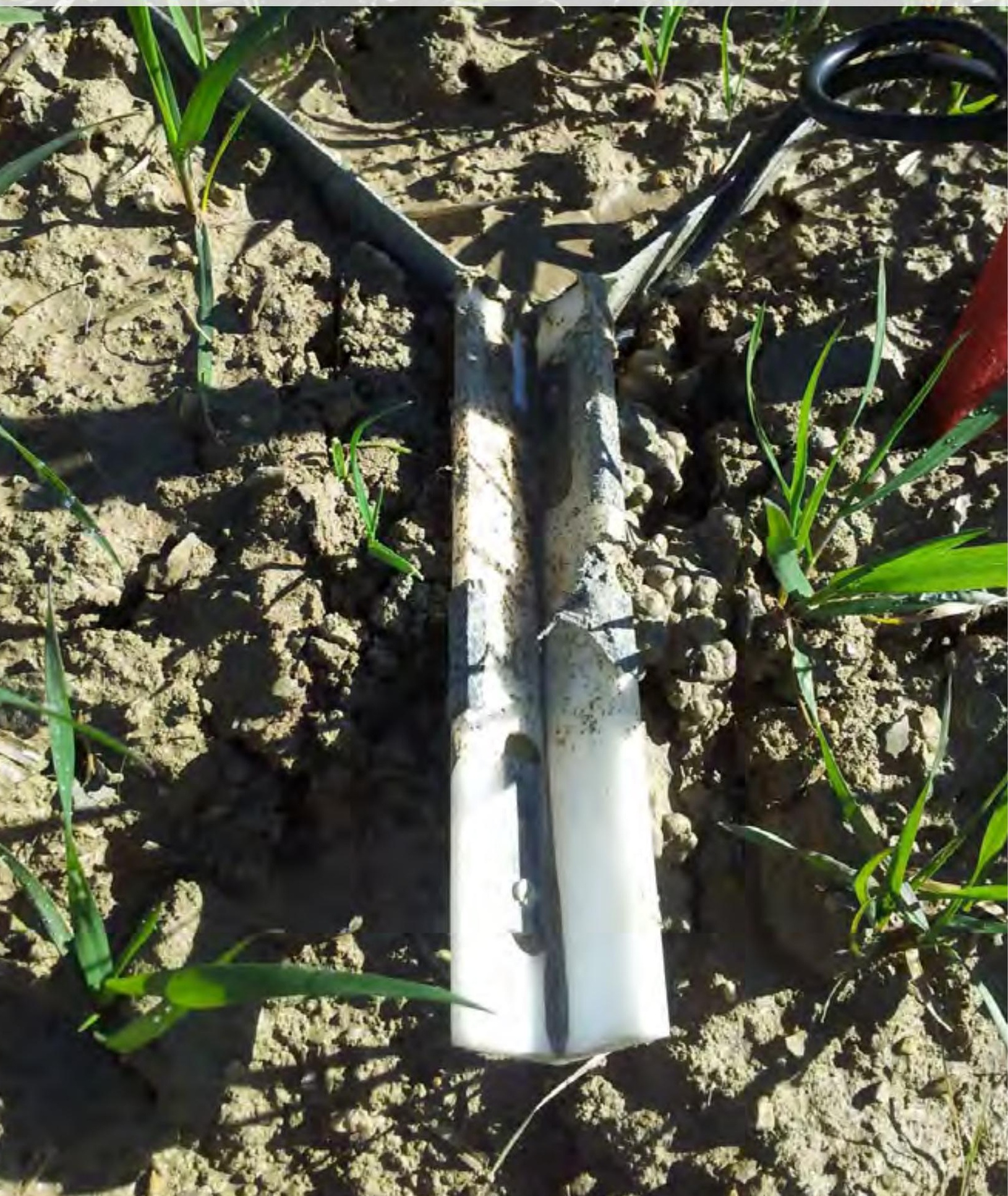

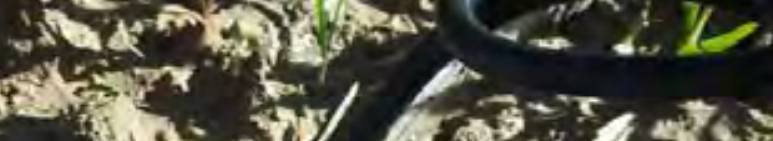

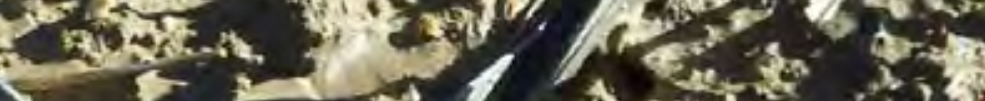

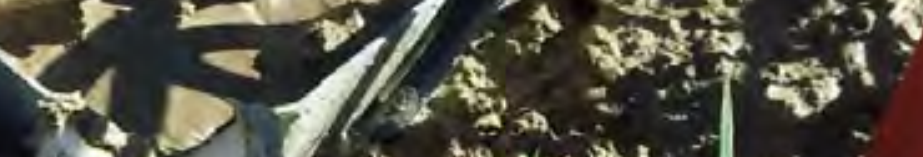

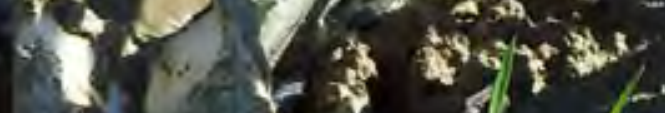
$y=x^{2}$ af (2),

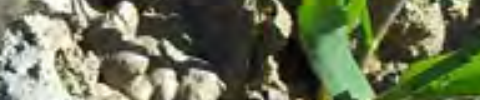
$g_{2}+x_{2}$

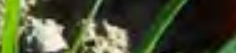

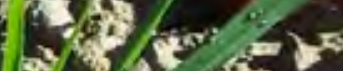

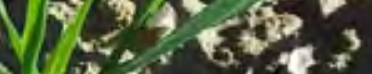

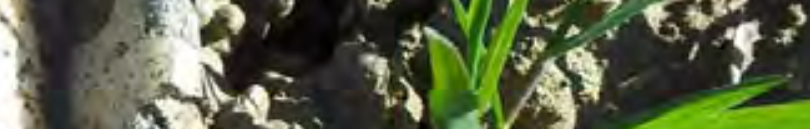

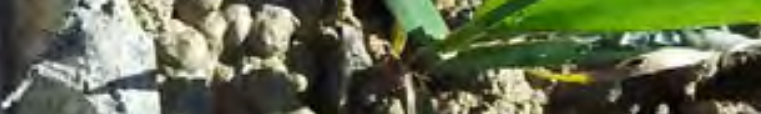

1.

is

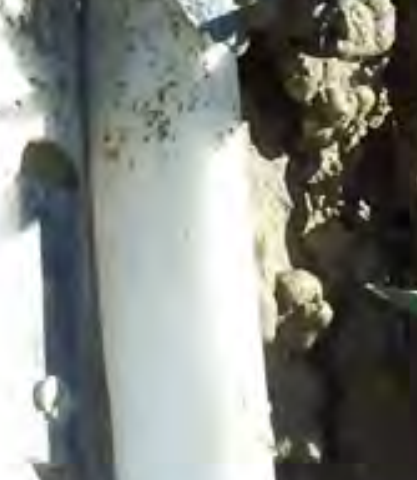

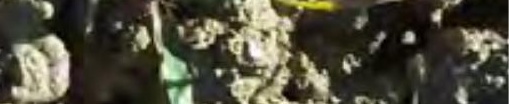

Sosition He. 2. $\mathrm{C}^{2}, \mathrm{~s}$

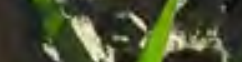

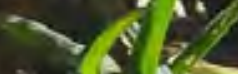
gas
$4 \mathrm{Me}_{\mathrm{S}} \mathrm{As}$

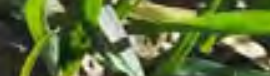

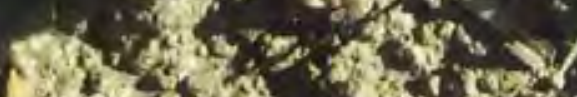

सी:

3 cindos

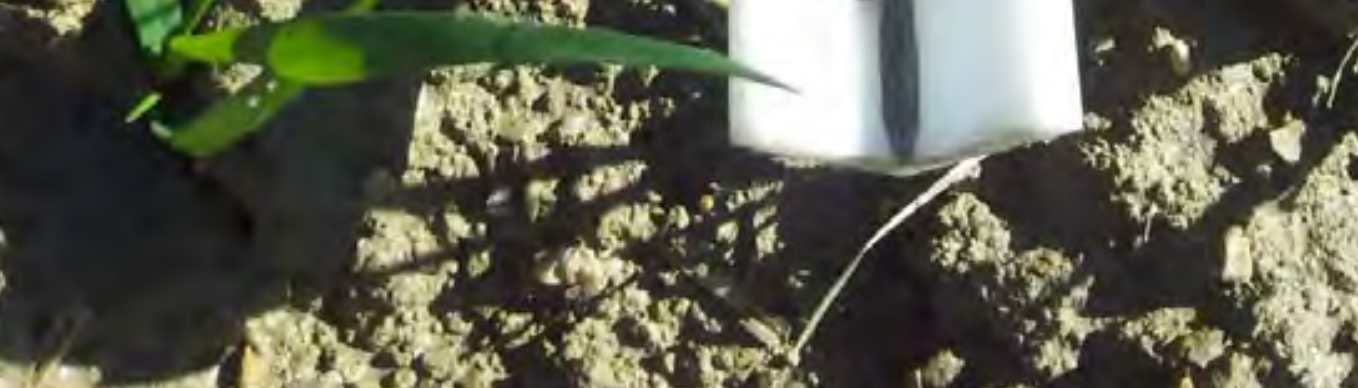

4.

\section{, 1 कर}




\begin{abstract}
Hydrological connectivity describes the physical coupling (linkages) of different elements within a landscape regarding (sub-) surface flows. A firm understanding of hydrological connectivity is important for catchment management applications, for e.g. habitat and species protection, and for flood resistance and resilience improvement(Bracken et al., 2013)(Bracken et al., 2013). Thinking about (geomorphological) systems as networks can lead to new insights, which has also been recognised within the scientific community, seeing the recent increase in the use of network (graph) theory within the geosciences. Network theory supports the analysis and understanding of complex systems by providing data structures for modelling objects and their linkages, and a versatile toolbox to quantitatively appraise network structure and properties. The objective of this study was to characterise and quantify overland flow connectivity dynamics on hillslopes in a humid sub-Mediterranean environment by using a combination of high-resolution digital-terrain models, overland flow sensors and a network approach. Results showed that there are significant differences between overland flow connectivity on agricultural areas and semi-natural shrubs areas. Significant positive correlations between connectivity and precipitation characteristics were found. Significant negative correlations between connectivity and soil moisture were found, most likely due to soil water repellency and/or soil surface crusting. The combination of structural networks and dynamic networks for determining potential connectivity and actual connectivity proved a powerful tool for analysing overland flow connectivity.
\end{abstract}

Published as: Masselink RJH, Heckmann T, Temme AJAM, Anders NS, Gooren HPA, Keesstra SD. 2017. A network theory approach for a better understanding of overland flow connectivity. Hydrological Processes 31 (1): 207-220. 


\section{Chapter 2. A network theory approach for a better understanding of overland flow connectivity}

\subsection{Introduction}

Hydrological connectivity describes the physical coupling (linkages) of different elements within a landscape regarding (sub-) surface flows (Pringle, 2003; Stieglitz, 2003; Croke et al., 2005). A firm understanding of hydrological connectivity is important for catchment management applications, for e.g. habitat and species protection, and for flood resistance and resilience improvement (Bracken et al., 2013).

Overland flow occurrence, quantities and connectivity are influenced by many factors, which have been studied in detail separately: e.g. topography (Cerdà and García-Fayos, 1997; Huang et al., 2002), plot size (Bryan and Poesen, 1989; Parsons et al., 2006), soil moisture (Ali and Roy, 2010), surface roughness (Darboux et al., 2002; Antoine et al., 2009; Trevisani and Cavalli, 2016) and vegetation (Bautista et al., 2007; Keesstra et al., 2012; Poeppl et al., 2012). Overland flow velocities and quantities have been measured in great detail in the field and the lab (Bren and Turner, 1979; Abrahams et al., 1986; Loch, 2000), and overland flow connectivity has been modelled (Heathwaite et al., 2005; Mueller et al., 2007; Lane et al., 2009). Overland flow connectivity measurements, however, have only been done in few studies (Cammeraat, 2004). Electrical resistance sensors can be used for the purpose of measuring overland flow connectivity.

Several studies have shown the suitability of electrical resistance sensors for determining the dynamics of stormflow generation (Srinivasan et al., 2002), for measuring the onset of ephemeral streamflow in wetlands (Goulsbra et al., 2009) and for assessing longitudinal connectivity in rivers (Jaeger and Olden, 2012). Variations in timing of overland flow generation have been studied by (Moody and Martin, 2015). Overland flow persistence and overland flow connectivity on hillslopes, however, have not yet been studied using these types of sensors. The sensors can provide valuable insights in the appearance of overland flow and its pathways. Electrical resistance sensors provide data at high spatial and temporal resolutions, which implies the analysis of large amounts of data. The analysis of these data can be facilitated by using a network approach.

Thinking about (geomorphological) systems as networks can often lead to new insights, which has been recognised within the scientific community as well, seeing the recent increase in the use of network (graph) theory within the geosciences (Heckmann et al., 2015; Phillips et al., 2015). Network theory supports the analysis and understanding of complex systems by providing data structures for modelling objects and their linkages, and a versatile toolbox to quantitatively appraise network structure and properties. Networks allow to "keep the whole in mind, whilst studying the parts and vice versa" (Jordán and Scheuring, 2004). As such, they form an intuitive toolbox to study connectivity, a system property that emerges from the interaction of the system's components (Heckmann et al., 2015). Network/graph theory has been used to study e.g. channel networks (Howard et al., 1970; Shreve, 1974; Werner, 1994; Marra et al., 2014), sediment connectivity (Heckmann and Schwanghart, 2013), sediment routing 
(Czuba and Foufoula-Georgiou, 2014), and runoff dynamics and connectivity in large catchments (Phillips et al., 2011). Few studies, however, focus on quantifying overland flow patterns on hillslopes.

The objective of this study was to characterise and quantify overland flow connectivity dynamics on hillslopes in a humid sub-Mediterranean environment by using a network approach. The sub-objectives were to:

- Create static and dynamic networks from local sensor measurements of surface runoff and high-resolution Digital Elevation Models.

- Apply graph-theoretic metrics to assess the hydrological connectivity on these networks, how the connectivity develops and changes over time and to select the most suitable metric for analysing overland flow connectivity.

- Quantify the differences between overland flow connectivity on hillslopes with agricultural use and those with (semi-) natural vegetation.

- Assess which factors influence overland flow connectivity on a hillslope and their importance.

- Distinguish the relation between overland flow connectivity at hillslope scale and discharge at catchment scale.

\subsection{Methods}

\subsubsection{Study Areas and data}

\section{Overland flow sensors}

Electrical resistance sensors were developed for the purpose of detecting the occurrence of overland flow at the point scale with high temporal resolution. The circuit design (Figure A1) is similar to the sensors developed by (Srinivasan et al., 2001). The physical design of the sensors was newly created to ensure optimal collection of overland flow through the sensor. The physical design consists of a $15 \mathrm{~cm}$ long v-shaped (15 x $15 \mathrm{~mm}$ ) Teflon piece, in which two slots of $15 \times 5 \mathrm{~mm}$ were milled on opposite sides and fitted with a brass alloy that withstands corrosion (Figure A2). The sensors were connected to a setup with a Campbell CR1000 datalogger in combination with a Campbell AM16/32B multiplexer. Up to 50 overland flow sensors and 4 soil moisture sensors were connected with this configuration. Electrical resistance through the two connectors was measured and logged every 10 seconds. Depending on the amount and the electrical conductivity of the water present between the connectors, the resistance approaches zero. Lab tests showed that the sensors were able to detect small amounts (flow height $<1 \mathrm{~mm}$ ) of rainwater with low electrical conductivity. A resistance was built into each of the sensors to check whether the cable from the datalogger to the sensor was not cut during agricultural practices, such as applying fertilizer/herbicides. The sensors were mounted on a v-shaped collector (Figure A2) of $25 \mathrm{~cm}$ wide to ensure that overland flow reached the sensor, even in low-flow situations. A thin "roof" of galvanised sheet metal was placed over the sensor to prevent direct precipitation from triggering it. Data from all overland flow sensors were checked for irregularities. Data from faulty sensors were removed from further analysis. The irregularities check also indicated that sensors on occasion suffered from false triggering, presumably because 
of dew formation. False positives resulting from this triggering were removed by setting a threshold of conductivity. This threshold was determined by taking the minimum value for each sensor during a 1-week dry period.

\section{Study area and overland flow data collection}

The study area "Latxaga" $\left(2.07 \mathrm{~km}^{2}\right)$ is situated in Northern Spain within the autonomous community of Navarre (Figure 2.1). Soils are finely textured, containing, on average, $10 \%$ sand, $60 \%$ silt and $30 \%$ clay. Mean annual precipitation is $835 \mathrm{~mm}$, the majority of which falls in winter. Land use in the catchment is mainly agriculture with dominantly cereal crops. Winter wheat (Triticum aestivum) and barley (Hordeum vulgare) are the most abundant crops. Some shrub areas exist on hillslopes, at locations where agricultural practice is impossible due to steep slopes or bedrock close to the surface (Casalí et al., 2008; Giménez et al., 2012b). Two hillslopes were chosen for overland flow data collection (Figure 2.1).

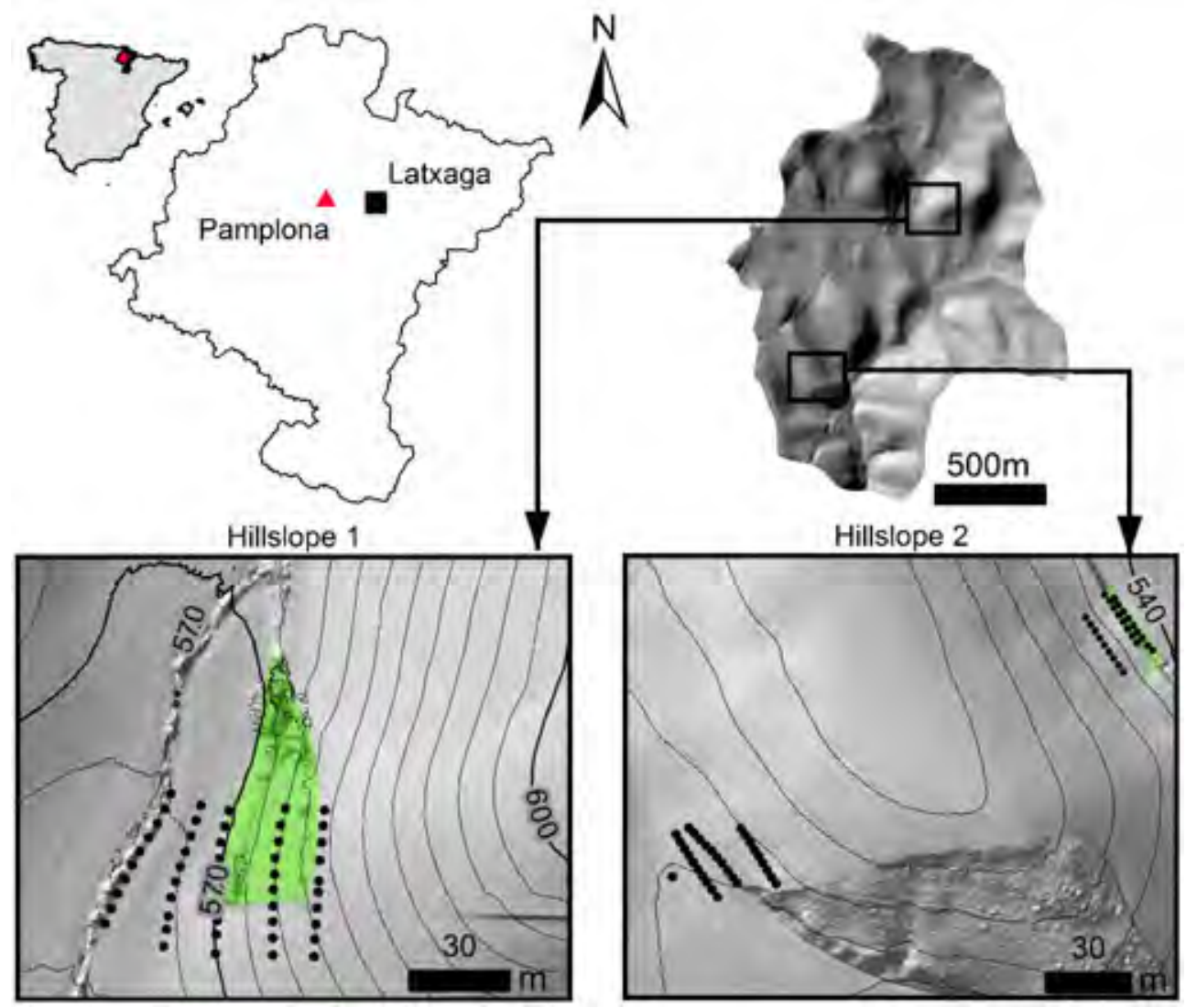

Figure 2.1 Two experimental hillslopes in the Latxaga catchment, Navarre. Spain. Dots on the lower figures represent the locations of the overland flow sensors. The shaded green areas represent the semi-natural shrub areas. 
Overland flow sensors were placed on hillslope 1 for two winters (2012-2013 and 2014-2015) and on hillslope 2 for the winter of 2013-2014. The reason for using two hillslopes was the rotation of crops in the winter of 2013-2014, which left hillslope 1 bare, with very high surface roughness caused by tillage operations. This high surface roughness causes additional ponding and allows more water to infiltrate and will, therefore, lead to less or no overland flow. Hillslope 2 was used instead for that winter. Hillslope 1 is divided into two sections: completely agriculture and agriculture with a shrubs strip. A total of 50 overland flow sensors was placed on hillslope 1 in February 2013 and in October 2014. Due to battery failure in 2014-2015 only the top 30 sensors provided data. At hillslope 2, two different sides were used to mimic the situation found at hillslope 1; a side with only agriculture (west) with 30 overland flow sensors and a side with both agriculture and a shrubs "strip" (east), also with 30 overland flow sensors (Figure 2.1). Hillslope 1 has an average slope of $25 \%$, and a length of $200 \mathrm{~m}$. Hillslope 2-W (agriculture) has an average slope of $23 \%$ and a length of $80 \mathrm{~m}$. Hillslope 2-E (shrubs) has an average slope of $27 \%$ and a length of $95 \mathrm{~m}$. Sensors were placed in a series of parallel rows to allow assessment of overland flow occurrence at equal altitudes over the slope. Four soil moisture sensors (Decagon EC-5) were also placed on each of the hillslopes to assess the influence of soil moisture for overland flow generation. Because of difficulties calibrating the sensors, and measuring errors in general (van den Elsen et al., 2014), the average of the four sensors was used as a single measure for soil moisture.

\section{Precipitation Events}

Precipitation data were available with a 10 minute interval, collected by a tippingbucket rain gauge, situated below hillslope 1 (Navarra, 2001). Precipitation events were automatically extracted by setting two rules: a minimum amount to start an event within an associated time period, and a minimum duration of a dry period to stop the event. The minimum amount for an event to start $(1 \mathrm{~mm}$ within $30 \mathrm{~min})$ and the duration of the dry period to stop the event $(6 \mathrm{~h})$ were chosen to ensure separation of individual small events.

\section{Digital Terrain Models}

In order to produce up-to-date, high-resolution Digital Elevation Models (DEMs) of the hillslopes, aerial photographs were taken using an Unmanned Aerial System (UAS) in February-March 2013, 2014 and 2015. The UAS used was a MAVinci Sirius fixed-wing aircraft, with on board a Panasonic Lumix GX1 16-megapixel camera. The ground sample distance was about $2 \mathrm{~cm}$ with a flight altitude of approximately $100 \mathrm{~m}$. The aerial photos were processed using Structure-from-Motion (SfM) stereophotogrammetry (Westoby et al., 2012) using the software Agisoft Photoscan Professional 1.1. The SfM photogrammetric processing produced 3D point clouds with an average point spacing of $0.06 \mathrm{~m}\left(272.59\right.$ points $\left./ \mathrm{m}^{2}\right)$ for $2013,0.11 \mathrm{~m}(84.9$ points $\left./ \mathrm{m}^{2}\right)$ for 2014 and $0.03 \mathrm{~m}\left(930\right.$ points $\left./ \mathrm{m}^{2}\right)$ for 2015 . Reasons for differences in the point spacing for the different years include different lighting conditions (low lighting meaning less points) and flight altitude (higher altitude meaning less points). The point cloud comprises both ground points and vegetation points. 
To ensure high accuracy of surface flow direction and contributing area estimates, we filtered out vegetation points to create a Digital Terrain Model (DTM). An iterative surface lowering procedure based on the ground point extraction from laser scanning data (Kraus and Pfeifer, 1998) was adjusted for use with photogrammetric point clouds(Anders et al., In prep). The procedure entails iteratively interpolating a surface from the point cloud and removing points above the interpolated surface (representing vegetation), after which a new interpolated surface is constructed. The procedure stops when the surface remains stable and all remaining points are considered ground points. The remaining points are then used to construct the final DTM.

The output cell size of the DTM was set to $25 \mathrm{~cm}$, which was considered detailed enough to register relatively small changes in e.g. surface roughness, and at the same time large enough to smooth out potential noise. Furthermore, choosing a cell size of $25 \mathrm{~cm}$ ensures the point spacing/cell size ratio is sufficiently low (Lucieer et al., 2011; Javernick et al., 2014), and constrains computation time of the DTM production and network analyses.

\subsubsection{Structural Network}

Overland flow sensor locations in combination with the high-resolution DTM served as a basis to construct the flow networks on the hillslopes. A Multiple-Flow-Direction (MFD) matrix M as proposed by (Schwanghart and Kuhn, 2010), was calculated from the DTM using the MFD algorithm (Freeman, 1991). The MFD algorithm distributes flow $(f)$ from each raster cell $i$ to a set of neighbours $(j$, limited to cells with a lower elevation), with a weight proportional to the slope $s$ to each of these neighbours raised to a power of $\rho$ :

$f_{i}=\frac{\max \left(0, s_{i}^{\rho}\right)}{\sum_{j=1}^{\delta} \max \left(0, s_{j}^{\rho}\right)}$

According to Freeman (1991), the default setting of $\rho=1.1$ gives the best results. The algorithm is appropriate for high-resolution DTMs of smooth topography, as it allows for divergent flow, albeit that some adjustments to the algorithm need to be made for optimal results (Endreny and Wood, 2003). The $\mathbf{M}$ matrix comprises $n$ rows and $n$ columns, where $n$ is the number of cells in the DTM. Elements in $\mathbf{M}$ contain the proportion of discharge $\mathrm{M}_{\mathrm{ij}}$ transferred from one cell $i$ to a maximum of eight downward neighbours with the index $j$. Then, using the procedure used by (Schwanghart and Heckmann, 2012), a probability matrix $\mathbf{P}$ of $n$ rows and $m$ columns was created, where $n$ is the total number of cells in the DTM and $m$ is the number of cells with sensors present $s=1,2, \ldots, m$. This matrix is constructed using the following matrix operation:

$\boldsymbol{P}=(\boldsymbol{I}-\boldsymbol{M})^{-1} \boldsymbol{S}$

where $\mathbf{I}$ is the identity matrix and $\mathbf{S}$ is a matrix with $\mathrm{n}$ rows and $\mathrm{m}$ columns that contains only zeroes and ones. Each column in $\mathbf{S}$ refers to a sensor and contains 1 non-zero entry at the location of the sensor. Each element in $\mathbf{P}$ now represents the probability $p(i, s)$ that element $i$ drains into $s$. This approach is equivalent to interpreting the area fractions computed by the MFD algorithm as probabilities, $p(i, s)$, and subsequently 
multiplying these probabilities along flow paths to calculate the probability that runoff is delivered from a cell to a sensor. The probability that a cell drains into a sensor is interpreted as an area fraction of this cell that delivers runoff to the sensor. The number of matrix fields with non-zero probabilities in a column of $\mathbf{P}$ (corresponding to a sensor $s$ ) represents the size of the total contributing area of that sensor, i.e. all cells from which (theoretically) the sensor can be reached. The column sum in $\mathbf{P}$ represents the effective contributing area, i.e. the probability of flow for each cell reaching the sensor interpreted as an area. These matrix operations are costly in terms of memory and computational power when using conventional matrices. By using sparse matrices, however, both the amount of memory and computational needs are greatly reduced, making the operations possible even for large DEMs ( $>50$ million cells).

The particular MFD algorithm used is known to introduce excessive dispersion because it distributes flow towards all lower neighbors, which causes the creation of pathways with extremely low probabilities that in real life would not exist at all (Tarboton, 1997). Following pathways with low probabilities (as opposed to the steepest descent rule for which the MFD algorithm generates the highest weights) allows for extreme divergence (Endreny and Wood, 2003) and leads to extremely low probabilities connecting, for example, a cell to a sensor. To reduce this excessive flow divergence, a threshold was applied to $\mathbf{P}$, below which the probability was set to zero. A range of thresholds for all years and all sensors was tested (Figure 2.2); while the number of non-zero matrix fields (i.e. the total contributing area) decreases exponentially with increasing thresholds, the effective contributing area (i.e. the sum of the probabilities within the contributing area) starts decreasing at a probability of $10^{-3}$. This probability value of $10^{-3}$ was chosen as a threshold, because then the divergent behaviour of the MFD algorithm is reduced, while maintaining all effectively contributing cells within the contributing area. Cells with a probability for reaching any sensor lower than the threshold probability were considered to be outside of the area of interest and, therefore, omitted from further analyses.

Our idea of a network model G(N,E) of overland flow involves: nodes $N$ representing parts of the hillslope (i.e. groups of pixels), and edges $E$ that represent surface runoff pathways between these areas (Figure 2.3). Nodes comprise the contributing areas (CA) of the sensors The CAs are delineated by assigning each raster cell to the sensor that its outflow is most likely to reach; cells with more than one none-zero entry in the corresponding row of the $\mathrm{P}$ matrix (when the cell is contributing to more than one sensor) belong to the CA of the sensor that has the highest probability of being reached.

The flow directions on the DTM define which downslope sensors can be reached from the $\mathrm{CA}$ of a particular sensor; these potential linkages represent the edges $\mathrm{E}$ of the network G, and the topology of these flow paths thus indicates structural connectivity. The sum of probabilities (in the P matrix) of a CA reaching a downslope sensor is used as a weight for the respective edge in G. This sum of probabilities can be considered as the area that drains into the downslope sensor in a fully saturated, connected, steadystate situation. The process is illustrated in Figure 2.3. 
In order to include all sensors in the analyses, an artificial "outlet node" was created. This outlet node represents a stream or gully where all overland flow from the hillslope flows to. Nodes that have no downslope connecting sensor were connected to this outlet node. The weight for edges leading to the outlet node was calculated as explained before.

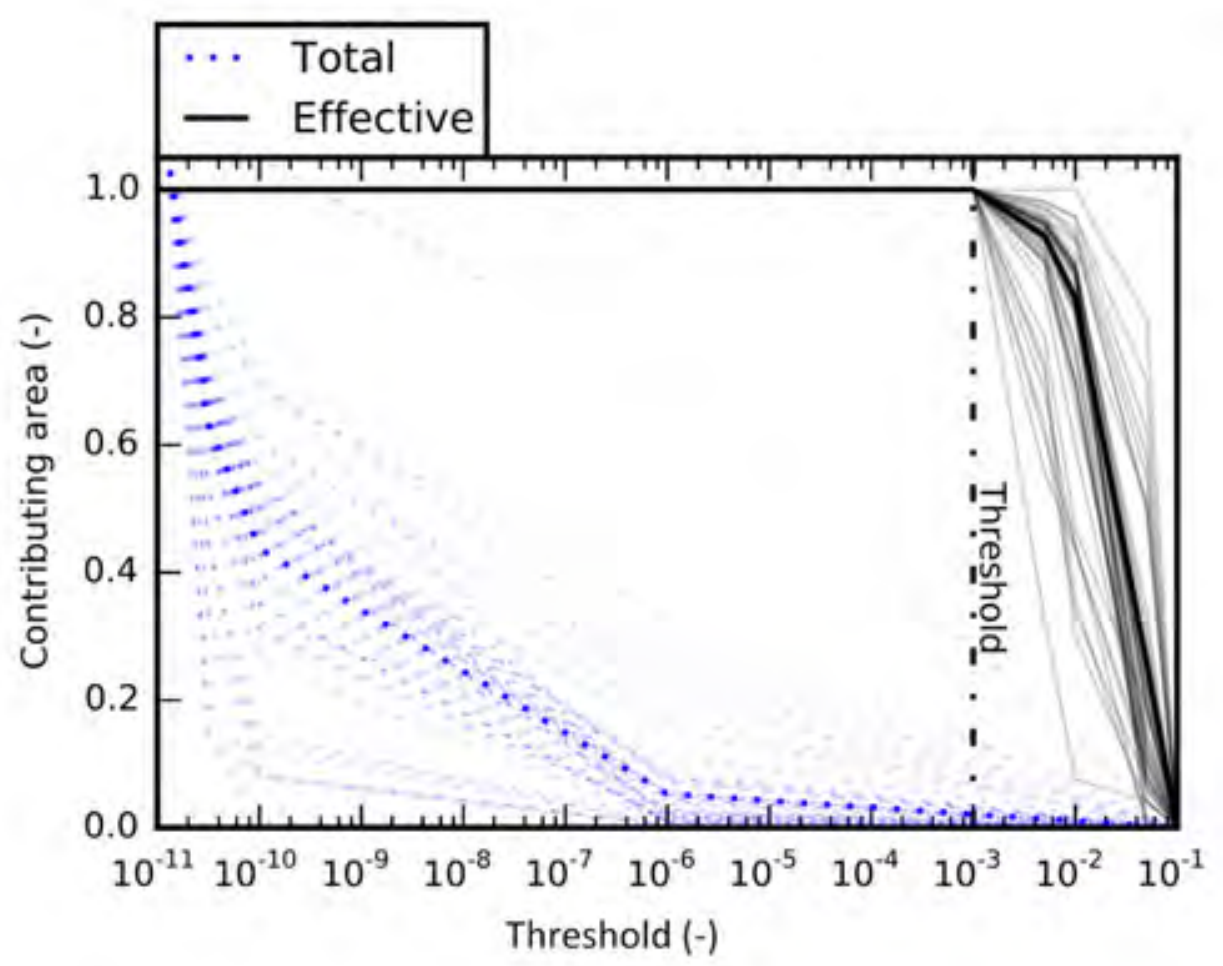

Figure 2.2 Relative total and effective contributing area for 48 sensors (thin lines) for 2013 and their means (thick lines). Effective contributing area is the sum of all probabilities of a contributing area. Threshold for omission of non-effectively contributing cells is depicted at $10^{-3}$.

\subsubsection{Network Analysis}

A network was created and analysed for every time step for the overland flow sensor data $(\Delta \mathrm{t}=10 \mathrm{~s})$. The structural network with edges removed served as a template. Whenever a sensor detected overland flow, all outgoing edges of the corresponding node of the structural network were added to the functional network for that time step.

Many network properties can be determined, with some differences between weighted and unweighted networks, and between directional and non-directional networks (Phillips et al., 2015). We first hypothesized how "high hydrological connectivity" would be reflected in network properties: in an unconnected system, no paths exist and all nodes are separate components. When edges are added to the network, the average path length increases, and by connecting nodes, the number of components decreases. 


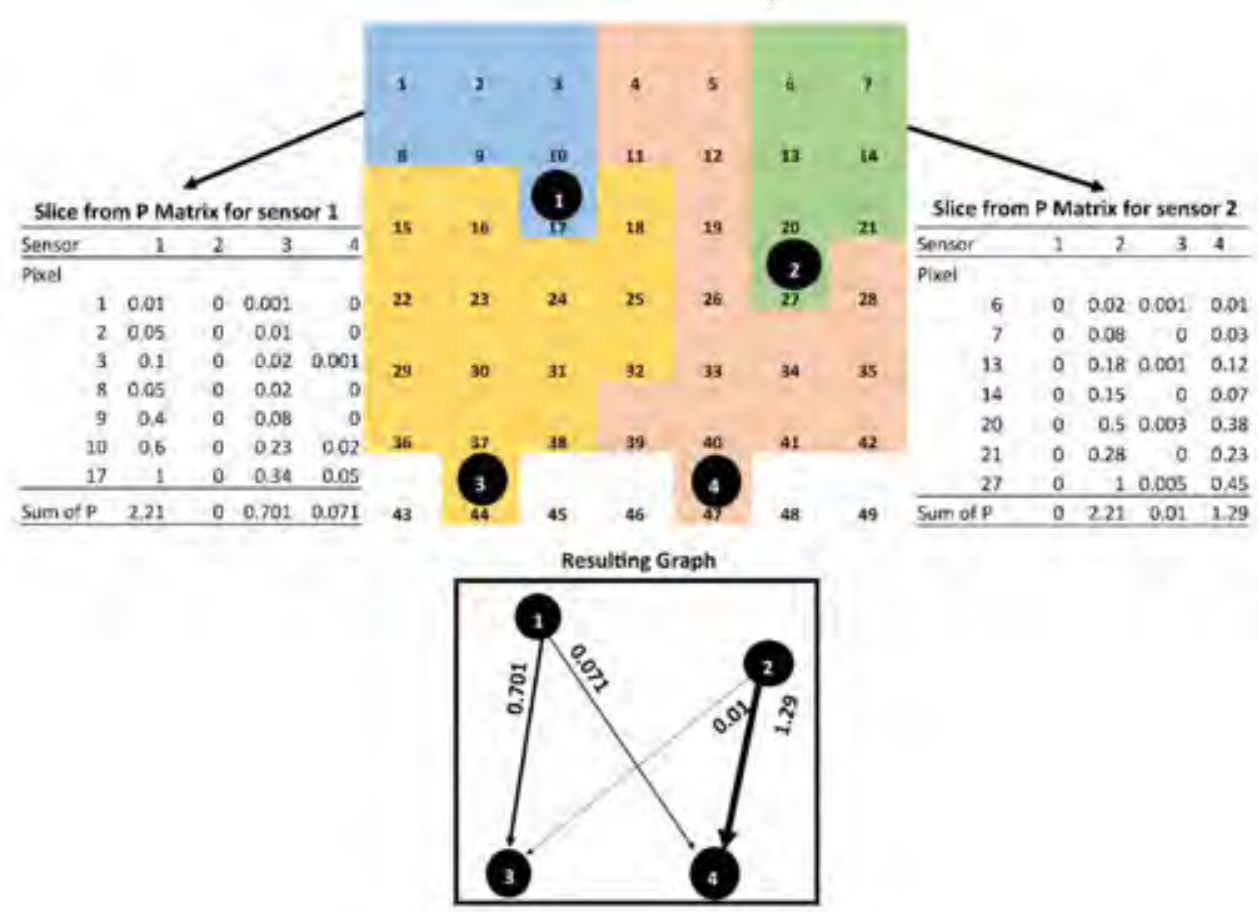

Figure 2.3 Schematic representation of the construction of the structural network from the contributing areas (CAs) for four sensors. Pixels are assigned to a sensor based on the highest probability. Connections only exist when a sensor is downslope of another sensor and their CAs overlap. The weight of an edge is the sum of probabilities for all cells in the CA to reach the downslope sensor.

Besides analysing the number of the average path length and components, other network properties, like network density (ratio between number of edges and nodes) were analysed. We verified our hypothesis that these network properties would be highly collinear (Table 2.1) and, therefore we decided to continue the analyses with a single network property: weighted network size, which is the sum of all edge weights of the network. The ratio between the sum of the edge weights per time step and the sum of edge weights of the completely connected system, i.e. the structural network was then used to analyse the system per time step. We defined this ratio as the "flux ratio". When the ratio equals zero, there are no connections. When the ratio equals 1 , it represents a fully connected hillslope in the sense that all topographically possible linkages are active. Note that this is not the same as full connectivity in graph theory, which denotes connections between all pairs of nodes. The combination of structure and function into a single, quantifiable measure for connectivity makes the flux ratio an informative and suitable network property.

To quantify differences between the agricultural areas and the areas that include shrubs, subnetworks were created for each area. For hillslope 1 this meant using the sensors within the shrubs area and the sensors that are upslope and downslope of the 
shrubs area as a subnetwork for the shrubs area. The remaining sensors were used as a subnetwork for the agricultural area.

For hillslope 2, a division already exists between the two sides of the hillslope and it was therefore not necessary to further subdivide these networks. Areas containing shrubs hereafter will be referred to as "shrubs areas" and the completely agricultural areas as "agricultural areas".

Table 2.1 Correlation Matrix of network properties for 2013. All values are highly collinear.

\begin{tabular}{lcccccc}
\hline & Average BC & Average PL $^{\mathrm{b}}$ & Components & Density & Edges $^{\mathrm{d}}$ & WNS $^{\mathrm{c}}$ \\
\hline Average $\mathrm{BC}^{\mathrm{a}}$ & 1 & & & & & \\
Average PL & 0.99 & 1 & & & & \\
Components & -0.99 & -0.98 & 1 & & & \\
Density & 1 & 0.99 & -0.99 & 1 & & \\
Edges $^{\mathrm{d}}$ & 1 & 0.99 & -0.99 & 1 & 1 & \\
WNS $^{\mathrm{c}}$ & 1 & 0.99 & -0.98 & 0.99 & 0.99 & 1 \\
\hline
\end{tabular}

a Average Betweenness Centrality

b Average Path length

c Weighted Network Size

d Number of Edges

For each precipitation event, the maximum flux ratio, mean flux ratio, time-to-peak (TTP) and a lag coefficient were calculated for both agricultural areas and shrubs areas. The TTP is defined as the time (in minutes) it takes from the start of the event to reach the maximum flux ratio for that event. The lag coefficient is defined as the ratio between the flux ratio at the end of the event ( 6 hours after the last rainfall) and the maximum flux ratio. The lag coefficient can be used to assess the duration of overland flow presence after an event has ended. A high lag coefficient means that overland flow persists after an event, whereas a low coefficient indicates that overland flow stops quickly after an event.

For each of the event properties a paired, one-tailed $t$ test was performed to test whether the means of maximum flux ratio, mean flux ratio and lag coefficient for the shrubs areas are equal to or higher than those of the agricultural areas $(\mathrm{p} \leq 0.05)$. Similarly, for TTP, whether mean TTP was equal or shorter for the shrubs area than for the agricultural area. To determine the effect size of the differences in the means of the samples, Cohen's d was calculated (Cohen, 1992).

The correlation between each of the event properties and influencing parameters was assessed using the Spearman's rank correlation coefficient. The influencing parameters that were used are the total precipitation ( $\mathrm{P}_{\text {Total }}$ ), maximum 10-min precipitation intensity ( $\mathrm{P}_{\operatorname{Max}}$ ), mean event precipitation ( $\mathrm{P}_{\text {Mean }}$ ) and antecedent soil moisture (volumetric water content: VWC). The correlation between the event properties on the hillslope and the catchment discharge (for details on discharge data collection see Casalí et al., 2008; Giménez et al., 2012) was also assessed by calculation of the Spearman's rank correlation coefficient. A Spearman's rank correlation was chosen 
instead of a Pearson correlation because of the non-normal distribution of the precipitation data and the high likelihood of non-linear correlations between the event parameters and the flux ratio parameters.

\subsection{Results}

A total of 89 events were extracted from the precipitation data of the three years, with total precipitation per event between 1.19 and $95.82 \mathrm{~mm}$ (Table 2.2). All 89 events were used in further analyses of the overland flow data.

The structural networks of the three years are depicted in Figure 2.4. The thickness of each edge indicates the edge weight, which can be interpreted as a flux $\left(\mathrm{m}^{2}\right)$ from a contributing area to a downslope sensor. The outlet nodes receive the highest fluxes in all cases, which is due to their position in the streams below the hillslopes.

Flux ratios for the agricultural areas are typically higher than flux ratios for the shrubs areas (shown for two examples in Figure 2.5). Flux ratios for the agricultural areas rise slightly earlier than flux ratios for the shrubs areas but variability during events is often similar between the agricultural and shrub areas. Lag coefficients in the two examples are similar; for both events the flux ratios have almost gone to zero 6 hours after the last precipitation.

Table 2.2 Five number summary for precipitation and soil moisture characteristics for all precipitation events used $(\mathrm{n}=89)$.

\begin{tabular}{lccccc}
\hline & Min & Q1 & Median & Q3 & Max \\
\hline Duration & 420 & 690 & 930 & 1500 & 5850 \\
Ptot $^{\mathrm{a}}$ & 1.19 & 3.96 & 7.88 & 17.05 & 95.82 \\
Pmax $^{\mathrm{b}}$ & 0 & 0.28 & 0.55 & 1.11 & 5.36 \\
VWC $^{\mathrm{c}}$ & 0.13 & 0.25 & 0.31 & 0.33 & 0.39 \\
\hline
\end{tabular}

a Total event preciptation

b Maximum 10-minute precipitation

c Antecedent volumetric water content $\left(\mathrm{m}^{3} / \mathrm{m}^{3}\right)$, measured at the start of the event.

Maximum flux ratio and mean flux ratio are lower for the shrubs areas than for the agricultural areas ( $<0.001$ for both cases see Table 2.3). The means for time-to-peak (TTP) and lag coefficients are not significantly different for the agricultural areas and shrub areas ( $p=0.28$ for TTP and $p=0.49$ for lag coefficients). Effect sizes in the form of Cohen's $d$ show that the differences between the means for the agricultural areas and the shrub areas for the maximum flux ratio $(\mathrm{d}=0.37)$ and mean flux ratio $(\mathrm{d}=0.57)$ range from medium to large.

Each of the precipitation parameters in table 2.4 shows significant correlations with the maximum flux ratio and mean flux ratio. Soil moisture, furthermore, shows a significant correlation with the mean flux ratio for both agricultural and shrubs areas. All precipitation parameters are positively correlated to maximum and mean flux ratio, whereas antecedent volumetric water content (VWC) in the soil is negatively 
correlated, especially for the shrubs areas. TTP for the agricultural areas is positively correlated to the total precipitation amount and the antecedent VWC. TTP for the shrubs area is correlated to the total precipitation amount and the mean precipitation amount. Lag coefficients for the shrubs areas are only significantly (negatively) correlated to the antecedent VWC. Lag coefficients for the agricultural areas are not significantly correlated with any of the parameters.

The maximum flux ratio of the agricultural areas is positively correlated to the maximum discharge and the Qmax/Qmean ratio (which indicates event peakedness), whereas the maximum flux ratio for shrubs is only correlated with the Qmax/Qmean ratio (Table 2.5). Mean flux ratio is only positively correlated to the $Q m a x / Q m e a n$ ratio for the agricultural areas. Furthermore, TTP is positively correlated with the maximum and mean discharge at the outlet and with the Qmax/Qmean ratio for both agricultural areas and shrub areas. The lag coefficient for the shrubs areas is negatively correlated to the maximum and mean catchment discharge. Remaining combinations of event characteristics and catchment discharge are very weakly correlated or uncorrelated.

Table 2.3 Means for all events ( $n=89)$ for the maximum flux ratios, mean flux ratios, Time-To-Peak (TTP) and lag coefficients for agricultural areas and shrubs areas. Cohen's d and associated p values for paired, twosample, one-tailed t-tests between the agricultural areas and shrubs areas are given in last row.

\begin{tabular}{lcccc}
\hline & \multicolumn{2}{c}{ Flux Ratio $(-)$} & \multirow{2}{*}{ TTPa $($ min) } & Lag Coeff. $(-)$ \\
\cline { 2 - 3 } & Max & Mean & & \\
\hline Agriculture & 0.39 & 0.58 & 499.21 & 0.45 \\
Shrubs & 0.33 & 0.47 & 519.66 & 0.45 \\
Cohen's d & $0.37(\mathrm{p}<0.001)$ & $0.57(\mathrm{p}<0.001)$ & $-0.06(\mathrm{p}=.28)$ & $0.00(\mathrm{p}=.49)$ \\
\hline
\end{tabular}



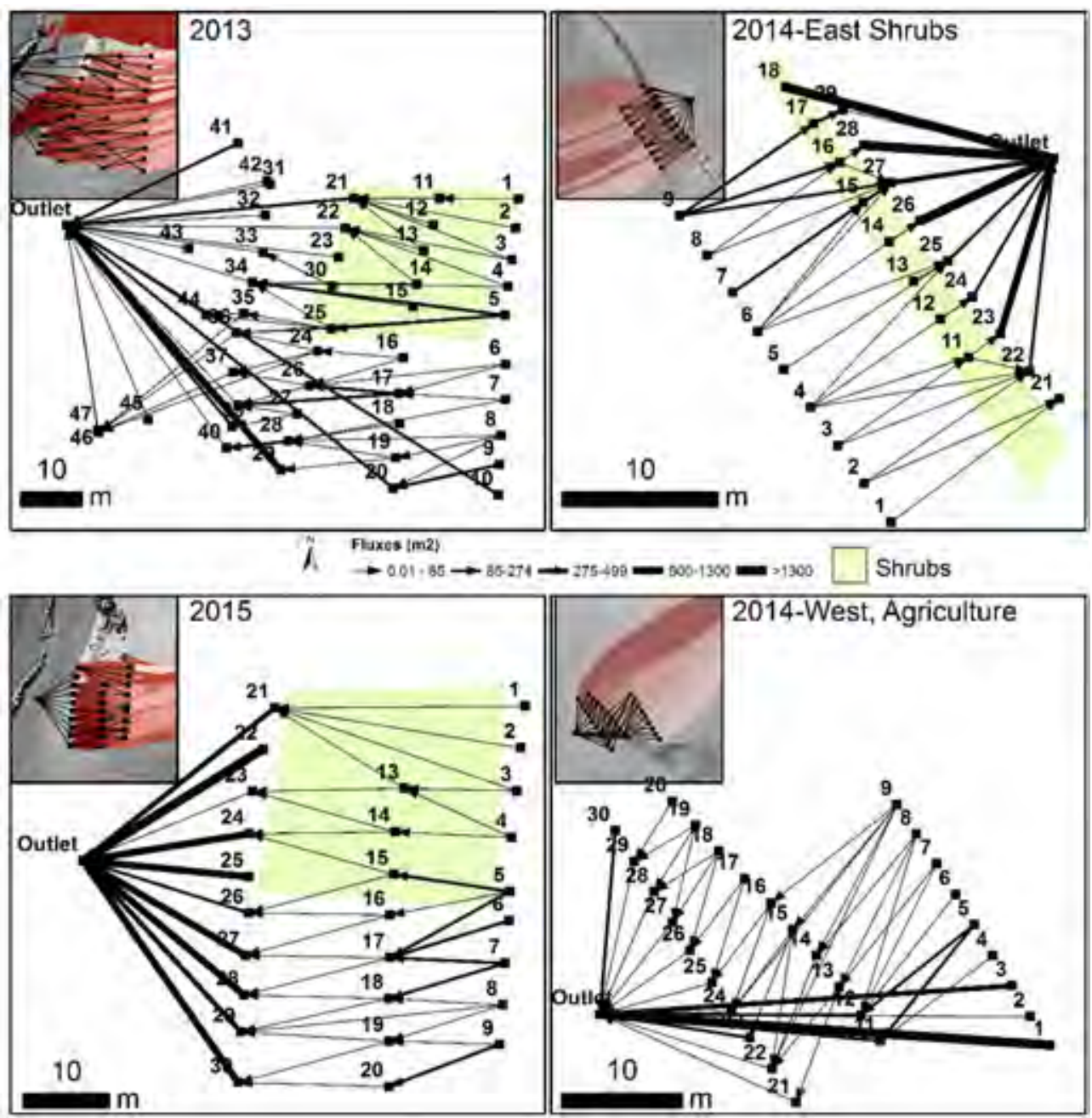

Figure 2.4 Structural networks for all three years, with 2014 being subdivided in an east (with shrubs) and a west (only agriculture) part. The insets show the networks on the hillslope with their corresponding contributing areas. 

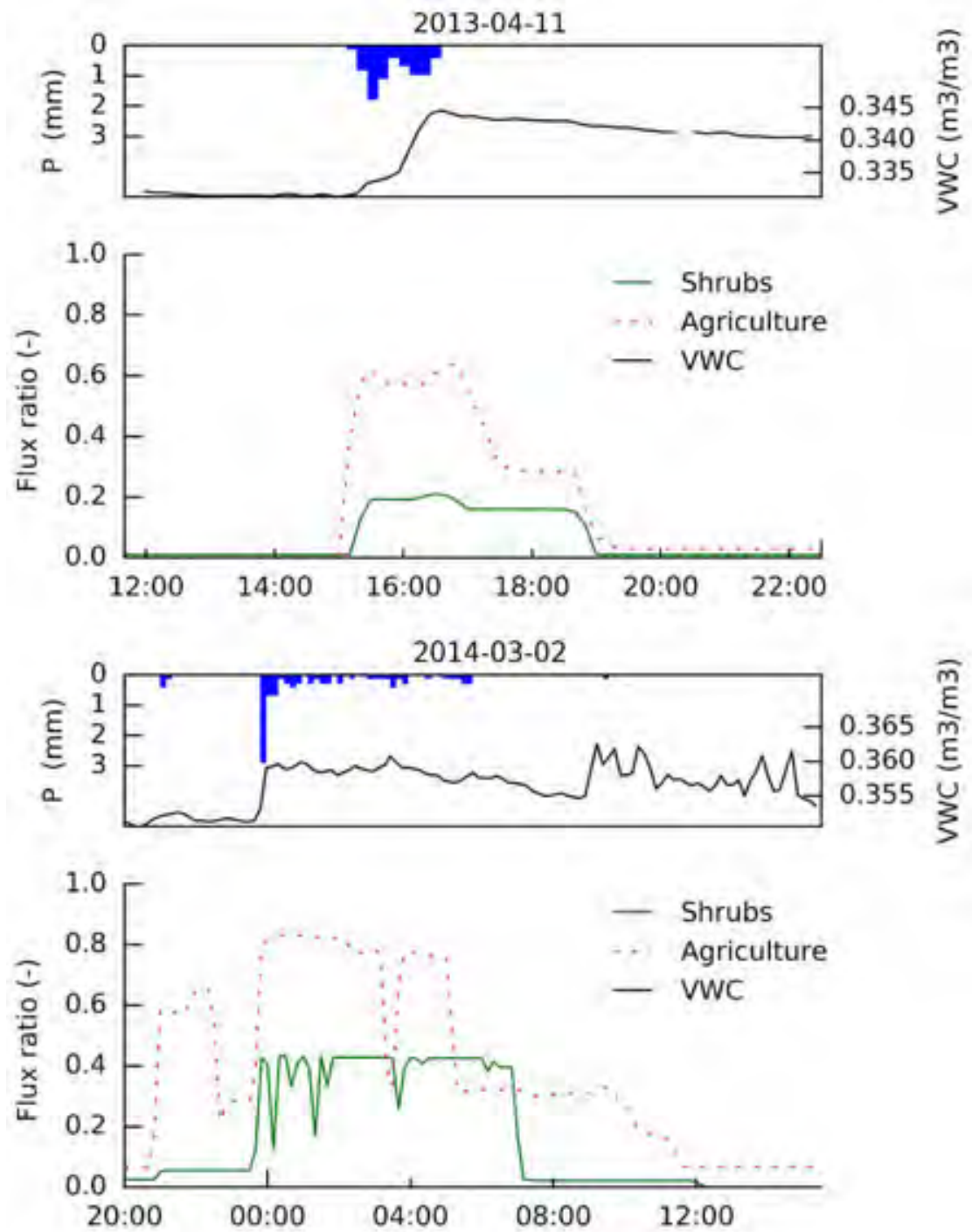

Figure 2.5 Two examples of precipitation mean antecedent soil moisture on the hillslope (volumetric water content, VWC) and flux ratios of the agricultural areas and shrubs areas for two precipitation events. 


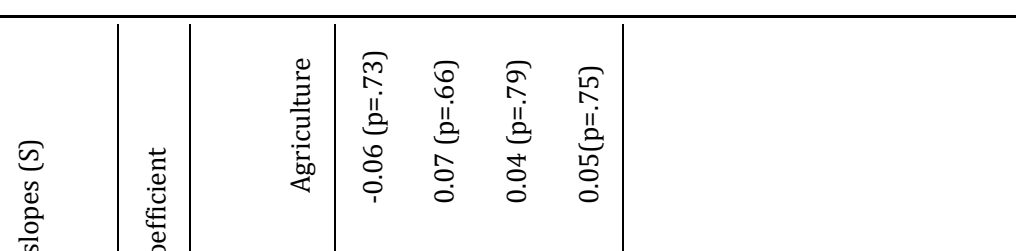

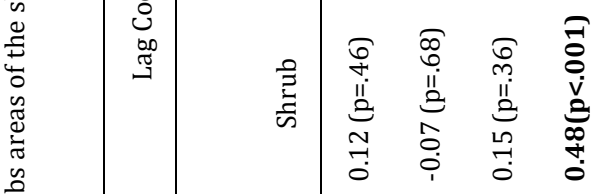

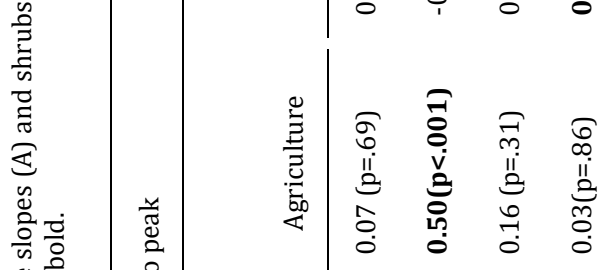

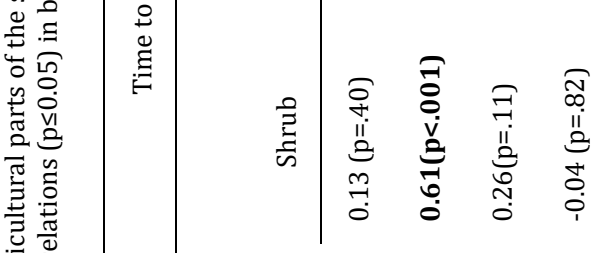

ธ్ర

क्ष

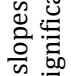

包

¿

ธี

:

ฮ

ธิ

कै

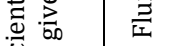

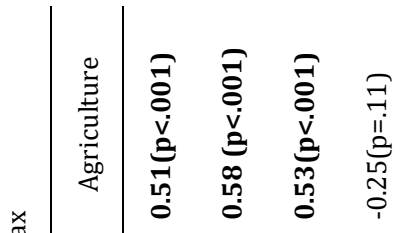

$\sum^{\pi}$

串

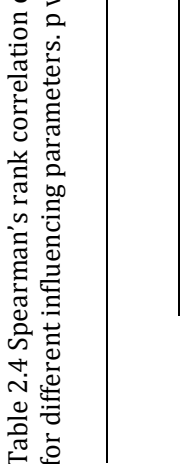

$\sum_{\Sigma}^{\approx}$

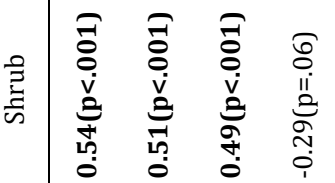

- 0 व

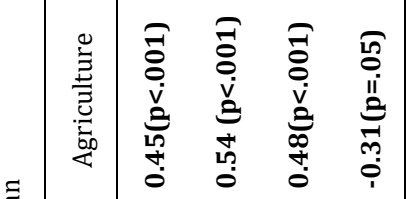

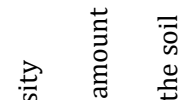

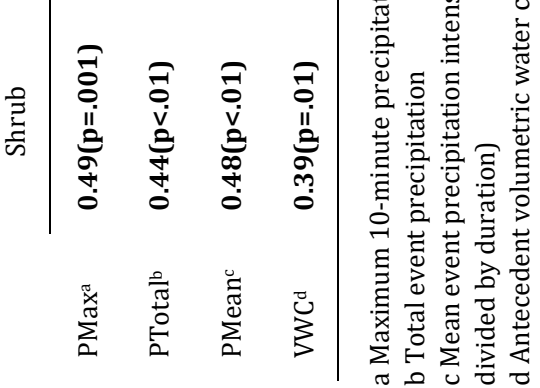


Table 2.5 Spearman's rank correlation coefficients between maximum and mean discharge at the outlet of the catchment for each event, the ratio between the maximum and mean discharge, and the network properties for each event for the agricultural (A) areas and shrubs (S) areas. p values are given between parentheses, significant correlations $(\mathrm{p} \leq 0.05)$ are in bold.

\begin{tabular}{|c|c|c|c|c|}
\hline & & Qmax & Qmean & Qmax / Qmean \\
\hline \multirow{2}{*}{ Max Flux Ratio } & A & $0.37(p=0.02)$ & $0.26(\mathrm{p}=0.10)$ & $0.35(p<0.001)$ \\
\hline & S & $0.28(\mathrm{p}=0.08)$ & $0.17(\mathrm{p}=0.29)$ & $0.24(p=0.03)$ \\
\hline \multirow{2}{*}{ Mean Flux Ratio } & A & $0.28(\mathrm{p}=0.08)$ & $0.18(\mathrm{p}=0.25)$ & $0.27(p=0.01)$ \\
\hline & S & $0.15(p=0.35)$ & $0.03(p=0.84)$ & $0.19(\mathrm{p}=0.08)$ \\
\hline \multirow{2}{*}{ Time to peak } & A & $0.34(p=0.02)$ & $0.31(p=0.05)$ & $0.33(\mathrm{p}=0.002)$ \\
\hline & S & $0.38(\mathrm{p}<0.01)$ & $0.38(p=0.01)$ & $0.38(\mathrm{p}<0.001)$ \\
\hline \multirow{2}{*}{ Lag Coefficient } & A & $-0.07(\mathrm{p}=0.67)$ & $-0.03(p=0.84)$ & $-0.09(\mathrm{p}=0.38)$ \\
\hline & $\mathrm{S}$ & $-0.37(p=0.02)$ & $-0.38(p=0.01)$ & $-0.15(\mathrm{p}=0.18)$ \\
\hline
\end{tabular}

\subsection{Discussion}

\subsubsection{Networks for the analysis of complex systems}

In this study, we have shown that connectivity of overland flow in a system can be quantified using a combination of a structural network and functional networks through time. The combination of a twofold network approach, i.e. a structural network and a functional network per time step provides valuable information on the functioning of the system at different locations on the hillslope. The approach of grouping pixels together into useful objects for the extraction of information belongs to a general trend in GIS, terrain analysis and remote sensing (i.e. Object-Based image analysis; (Anders et al., 2013; Wickama et al., 2015). This approach consists of creating functional objects that represent the space as closely as possible in relation to the processes involved, thus avoiding a single cell-based discretization with topographic attributes (Gascuel-Odoux et al., 2011).

The network approach allows us to analyse (i) single nodes, (ii) edges, (iii) edge sequences and (iv) properties of the whole network at the same time. This is in line with the statement by (Jordán and Scheuring, 2004), who mention that networks allow to "keep the whole in mind, whilst studying the parts and vice versa". In this study, we mainly looked at the properties of the whole network, while more information about connectivity might be extracted when looking at the individual components within the network.

The structural network can be analysed for the potential connectivity of topographydriven flow pathways in a system, while the functional networks depict the actual connectivity at a certain time step as a function of landscape parameters and external forcing. Other useful information can be extracted from the obtained data from the overland flow sensors: e.g. whether overland flow originates at the bottom of the slope 
or at the top (Moody and Martin, 2015), the response of the areas to consecutive rainfall events, the influence of slope angle (Abrahams et al., 1988), hillslope fragmentation (Ziegler et al., 2004) and the effects of vegetation growth (Wainwright et al., 2000) on overland flow connectivity. All these analyses were, however, beyond the scope of the current study. For analysing the effect of vegetation growth, furthermore, more data are needed to account for the variability of antecedent conditions and event magnitude.

\subsubsection{Factors influencing overland flow connectivity on a hillslope}

The flux ratio gives insight about the maximum connectivity on hillslopes under certain conditions, which could prove valuable for e.g. model input or calibration, estimating peak discharges and, hence, sediment yields. Results show that flux ratio maxima and means are, on average, substantially higher for agricultural areas than for shrubs areas (Table 2.3). There is very little ground cover in agricultural areas, causing less resistance to overland flow, causing higher velocities than in shrubs areas and, therefore, less time for infiltration into the soil (Esteves et al., 2000). The effect size of the $t$-test for maximum flux ratio $(\mathrm{d}=0.37)$ is smaller than the effect size of the $t$-test for the mean flux ratio $(d=0.57)$. We propose that this difference is because maximum connectivity often occurs at moments with high precipitation intensities and might be mainly a result of infiltration-excess overland flow. During events with very high precipitation intensities, the flux ratio maxima of the agricultural areas and shrub areas will both approach or equal 1, making the maximum flux ratios more similar than the mean flux ratios of an event.

Lag coefficients and time-to-peak (TTP) for both areas are similar, which shows that the areas react similarly to precipitation in regards to reaching peak connectivity and once precipitation has stopped, overland flow continues to approximately the same degree in both agricultural areas and shrubs areas. For some precipitation events, however, there is a difference in the lag between the agricultural and shrub areas, although this might not always be reflected in the lag coefficient, because this happens in the $6 \mathrm{~h}$ period just after the event (Figure 2.5 below). The differences in lag coefficients during events can be attributed to antecedent wetness, which shows a moderate, negative correlation with lag coefficients for shrubs, but not with agricultural areas (Table 2.4).

Analyses of influencing parameters on overland flow connectivity on the hillslopes (Table 2.4) show flux ratio is positively correlated to precipitation parameters. Maximum and mean flux ratios show a negative relation with antecedent soil moisture (Table 2.4): drier soils result in more overland flow. This contrasts with previous studies that showed that little to no surface runoff occurs for low soil moisture conditions (even for high precipitation intensities) by using a catchment-scale discharge model (Masselink et al., 2016) and using a rainfall simulator (Giménez et al., 2012b). The rainfall simulator experiment in the latter study was carried out in August on an extremely dry soil on a $1 \mathrm{~m}^{2}$ plot, while the overland flow sensors were installed during winter/spring on an entire hillslope. The overland flow sensors record overland flow at small spatial scales, and, therefore, are likely to detect overland flow sooner than 
experiments done at a plot or catchment scale, because at larger scales overland flow has more possibilities for reinfiltration due to variations in e.g. roughness and soil moisture. The negative relation between the connectivity on the hillslope and soil moisture could be caused by soil water repellency or crust formation. Crust formation occurs in the study areas, albeit only for the agricultural areas; the shrub areas have no (or very limited) crusting. This makes it unlikely that crusting causes the negative correlation between antecedent soil moisture and connectivity because this relationship is present for both agricultural and shrub areas. Data on soil water repellency was not available and, therefore, this could not be verified. Similarly, antecedent soil moisture and lag coefficient are negatively correlated. Antecedent soil moisture and the time-to-peak (TTP) are positively correlated for the agricultural areas, but not for the shrubs areas (Table 2.4). This indicates that higher antecedent soil moisture causes a relative delay in the peak of an event, because the connectivity is lower.

Other factors that might influence overland flow connectivity, but that were not included in the study include e.g. rock cover and surface roughness, although these could play a large role in the onset of overland flow and its flow paths (Bunte and Poesen, 1993; Cerdá, 2001; Darboux et al., 2002; Peñuela et al., 2015). Rock cover in the study area is relatively low and will, therefore, probably not play a big role in the overland flow connectivity development in the study area.

The presence of a significant relation between the maximum flux ratio and the catchment scale discharge for the agricultural areas and the absence of such a significant relation for the shrubs areas (Table 2.5) can be explained by the land use ratios in the catchment. The agricultural areas cover $\sim 90 \%$ of the catchment, while only $5 \%$ is covered with shrubs. The positive correlation between Qmax/Qmean ratio (event peakedness) and maximum flux ratio for both agricultural areas and shrub areas indicate that high hillslope connectivity indicates higher relative discharges peaks. The positive correlation between the TTP and the discharge is most likely due to moderate to strong correlations between TTP and total precipitation, which is also one of the main determinants in the maximum and total amount of discharge.

\subsubsection{Notes on methods and data}

Spatial resolution and temporal resolution of the overland flow data presented here are high compared to previous studies (Cerdà and García-Fayos, 1997; Cammeraat, 2004). However, overland flow is still measured at the point scale, which means that no information about overland flow between the sensors is available. This lack of information can be minimised by installing more sensors, although this implies more alterations to e.g. surface roughness. For a better understanding of the overland flow on the hillslope in general, an overland flow model could be applied to the hillslopes and validated with data from overland flow sensors (Coulthard et al., 2012). By assuming that an entire contributing area is active when a sensor is active, we overestimate the presence of overland flow on the hillslope, even though this is partly reflected in the edge weights. In this study, however, we mainly looked at differences 
between overland flow in semi-natural shrub areas and agricultural areas, which are equally affected by this overestimation of overland flow occurrence.

The overland flow sensors were installed in the field and checked only 2-3 times during each winter. Some sensors accumulated some sediment, which might have caused higher lag coefficients because wet sediment conducts electricity after water flow has stopped. This sediment accumulation, however, was present in only 3-5 sensors and, therefore, the effect on total lag coefficients of the entire hillslope has been minimal.

The removal of vegetation from the point cloud to obtain a Digital Terrain model (DTM) proved to be a challenging task, particularly in areas with dense shrubs. In these denseshrub areas the ground surface is insufficiently visible on the aerial photographs, resulting in very few ground points. Isolated shrubs could be filtered out, but extended patches of shrubs were incorrectly registered as ground surface. The vegetation that was not removed from the DSM caused a different delineation of contributing areas for the sensors and may, therefore, have had an influence on the final results. While laser altimetry would provide more ground points inside these shrub patches and produce more accurate DTMs, available laser scans for this area have too coarse resolution $(0.5$ point $/ \mathrm{m}^{2}$ ) and are not available for the years when the sensors were installed. Despite some left-over vegetation patches, we believe that the relatively high spatio-temporal resolution of UAS DTMs matches the scale of the sensor measurements better than existing laser scanning data. With UAS DTMs it is possible to delineate small scale features that have a large influence on overland flow processes, e.g. tractor track and rills (Basher and Ross, 2001; Kaspar et al., 2001a), while with the existing airborne laser-scanning DTMs this would not be possible.

The landscape changes continuously, and with it, the connectivity changes (LópezVicente et al., 2016; Peñuela et al., 2016).The DTMs only provide a snapshot of the connectivity at a certain time step within the measuring campaigns, which means that the structural connectivity of the system before and after the DTM data collection was different. Therefore, for future studies ideally, a new DTM should be created after every precipitation event to take the changes of structural connectivity into account.

Structural connectivity was determined by topography only, while actual structural connectivity is also governed by e.g. infiltration, roughness and vegetation (Wainwright et al., 2011). The measured signal from each of the sensors is, therefore, influenced by all these factors. We assumed that these differences in infiltration and roughness are mainly dominated by land use and are, therefore, also reflected in the results showing the difference between the shrubs areas and agricultural areas.

\subsubsection{Recommendations for future studies on connectivity using networks}

Networks have been used for decades to describe traffic flow (Riedel and Brunner, 1994), landscape connectivity in ecology (Bunn et al., 2000) and ecosystem management structures (Kininmonth et al., 2015). Networks are being used more frequently over the past years to study problems in earth sciences, providing insights into the functioning of complex systems (Heckmann et al., 2015; Phillips et al., 2015). 
All these studies demonstrate the utility networks can provide in getting a better understanding of complex systems and processes. We believe, therefore, that networks can and should be used more often in hydrological studies, especially those looking at hydrological and sediment connectivity. We can learn from other fields, e.g. by applying methods that have been successfully applied to ecological studies (Urban and Keitt, 2001). Some suggestions for future studies include the use of graph motifs as "building blocks" to describe catchments, river reaches or hillslopes, similar to the study of wetland management structures done by (Kininmonth et al., 2015). Other possibilities include combining an automated unsupervised classification method (Anders et al., 2011; Jasiewicz and Stepinski, 2013) with networks for modelling of overland flow and sediment transport, or creating "landscape graphs" for the detection of different geomorphological features within a landscape (Cantwell and Forman, 1993).

As noted already by Heckmann et al. (2015), using dynamic networks has great potential for determining and understanding sediment delivery within river reaches by representing them as a network with nodes representing sources, junctions and the outlet, which are connected by edges representing river segments (Czuba and FoufoulaGeorgiou, 2014). This type of approaches can also be applied to an entire catchment to improve our understanding of processes involved in sediment delivery from the hillslopes to the rivers and to improve our prediction models. Modelling approaches could, furthermore, benefit from using networks, as other examples from ecology have shown. Networks have been used for e.g.: modelling the dispersal of coral larvae between reefs using an Eulerian advection-diffusion approach (Treml et al., 2008), minimising data requirements for population modelling (Minor and Urban, 2007) and efficiently modelling connectivity on very large networks with millions of nodes and connections by using in electrical circuit theory (McRae et al., 2008).

\subsection{Conclusion}

In this study, we used a combination of overland flow data at high spatial and temporal resolution, high-resolution digital terrain models and networks to quantify overland flow connectivity and to determine factors influencing overland flow connectivity dynamics. The results showed that there are significant differences between maximum and mean overland flow connectivity on agricultural areas and semi-natural shrubs areas. Positive correlations between connectivity and precipitation characteristics were found, while negative correlations between connectivity and soil moisture were found, probably due to soil water repellency. The combination of structural networks and dynamic networks for determining potential connectivity and actual connectivity proved a powerful tool for analysing overland flow connectivity. We advocate the use of networks for studying complex hydrological and/or geomorphological systems/processes. 


\section{Acknowledgements}

This project was funded by the Dutch Organisation for Scientific Research (NWO) grant number 822.01.004 and by a Short-Term Scientific Mission of COST action ES1306: Connecting European Connectivity Research. We thank Hennie Gertsen for his help with the figure of the electrical scheme of the sensors. Finally, we would like to thank Manuel Seeger from the University of Trier for assisting us in finding appropriate sites for placing the sensors and Javier Casalí, Rafael Giménez, Alejandro Martínez de Aguirre and Alvaro Ollo from the Public University of Navarre, Spain, for giving logistical support and helping with the fieldwork 


\section{Chapter 3}
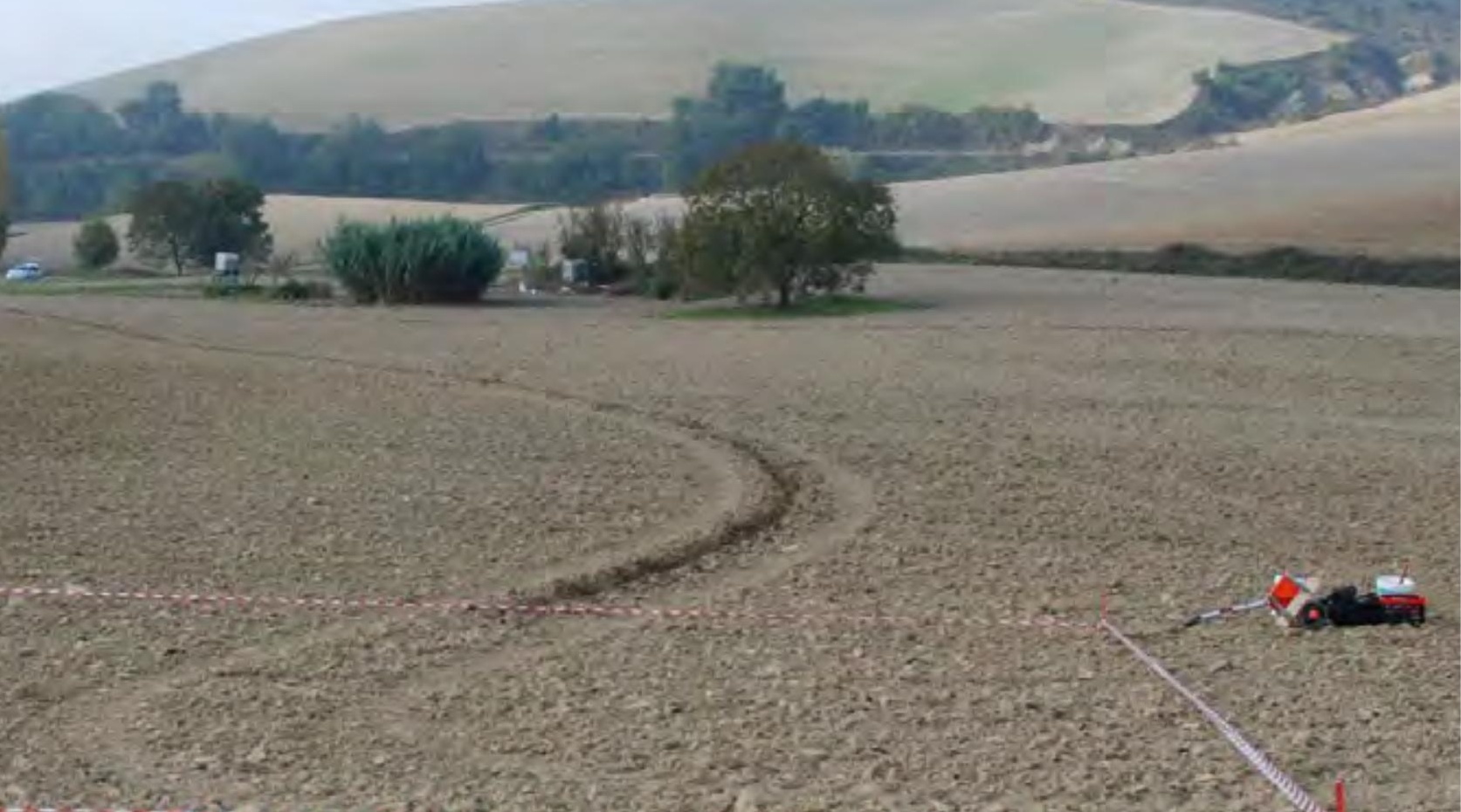

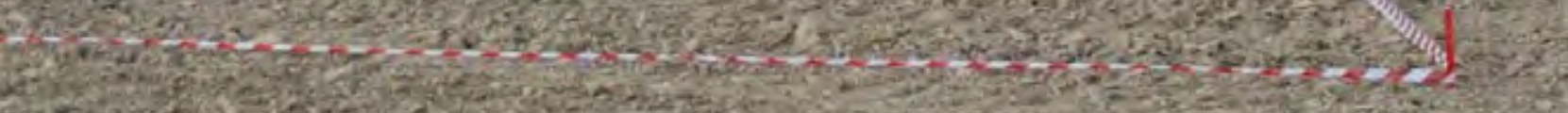

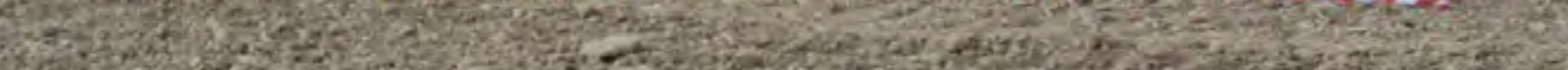
1.5,

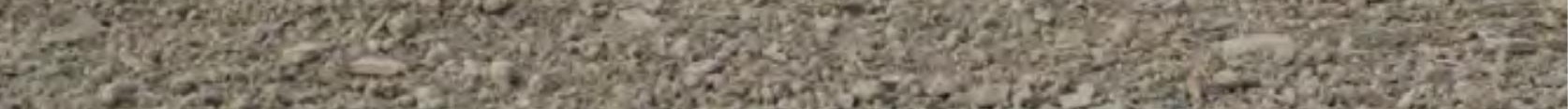

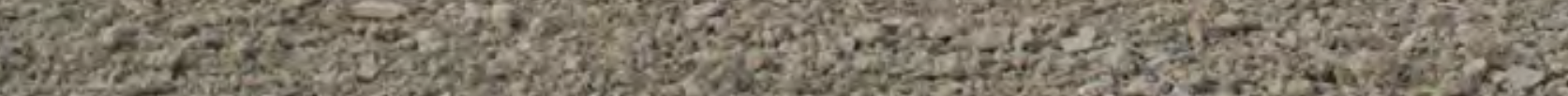

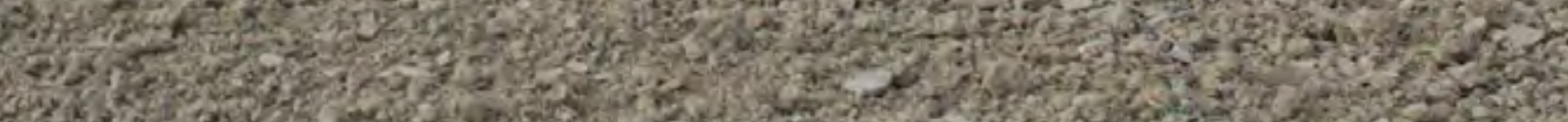

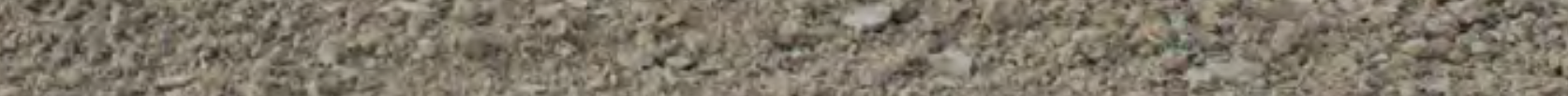
2.0.

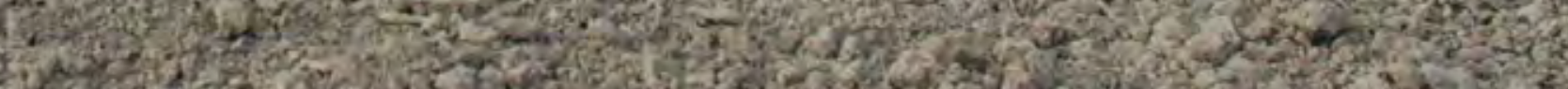

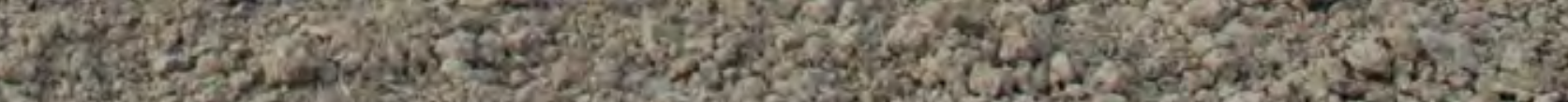

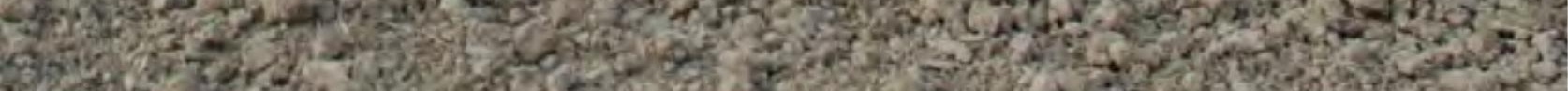

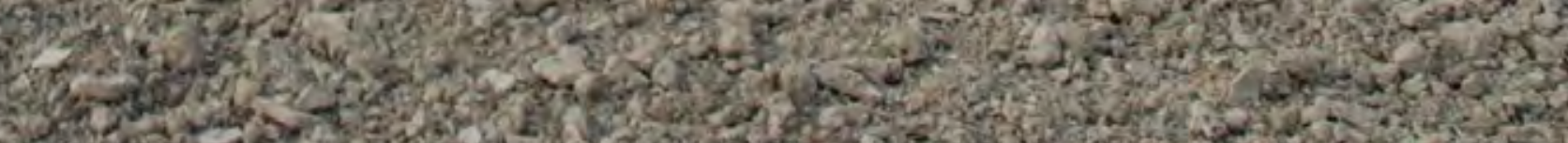

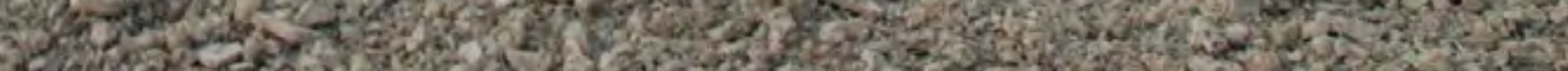

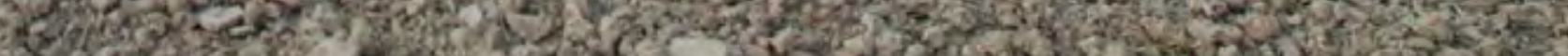

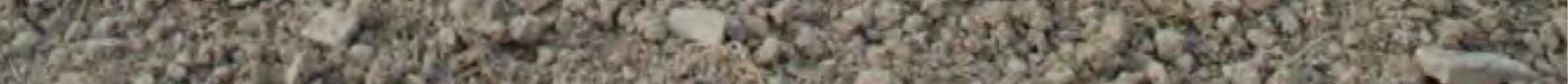

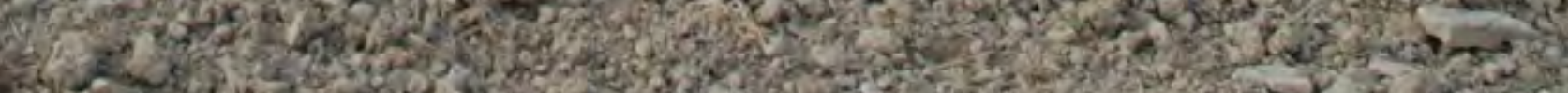

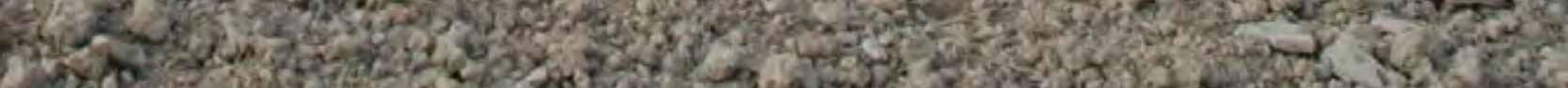




\begin{abstract}
Soil erosion from agricultural areas is a large problem, because of off-site effects like the rapid filling of reservoirs. To mitigate the problem of sediments from agricultural areas reaching the channel, reservoirs and other surface waters, it is important to understand hillslope-channel connectivity and catchment connectivity.

To determine the functioning of hillslope-channel connectivity and the continuation of transport of these sediments in the channel, it is necessary to obtain data on sediment transport from the hillslopes to the channels. Simultaneously, the factors that influence sediment export out of the catchment need to be studied.
\end{abstract}

For measuring hillslope-channel sediment connectivity, Rare-Earth Oxide (REO) tracers were applied to a hillslope in an agricultural catchment in Navarre, Spain, preceding the winter of 2014-2015. The results showed that during the winter no sediment transport from the hillslope to the channel was detected.

To test the implication of the REO results at the catchment scale, two contrasting conceptual models for sediment connectivity were assessed using a Random Forest (RF) machine learning method. The RF method was applied using a 15 year period of measured sediment output at the catchment scale. One model proposes that small events provide sediment for large events, while the other proposes that only large events cause sediment detachment and small events subsequently remove these sediments from near and in the channel. For sediment yield prediction of small events, variables related to large preceding events were the most important. The model for large events underperformed and, therefore, we could not draw any immediate conclusions whether small events influence the amount of sediment exported during large events.

Both REO tracers and RF method showed that low intensity events do not contribute any sediments from the hillslopes to the channel in the Latxaga catchment. Sediment dynamics are dominated by sediment mobilisation during large (high intensity) events. Sediments are for a large part exported during those events, but the system shows a memory of the occurrence of these large events, suggesting that large amounts of sediments are deposited in and near the channel after these events. These sediments are gradually removed by small events. To better understand the delivery of sediments to the channel and how large and small events influence each other more field data on hillslope-channel connectivity and within-channel sediment dynamics is necessary.

Published as: Masselink R.J.H, Temme AJAM, Giménez R, Casalí J, Keesstra SD. 2017. Assessing hillslopechannel connectivity in an agricultural catchment using rare-earth oxide tracers and random forests models. Cuadernos de Investigación Geográfica 43 (1): 17-39. 


\section{Chapter 3. Assessing hillslope-channel connectivity in an agricultural catchment using rare-earth oxide tracers and random forest models}

\subsection{Introduction}

Soil erosion in agricultural areas is a large problem worldwide, because of a loss of productivity (Cerdà et al., 2009; García-Orenes et al., 2009), but also because of off-site effects like the rapid filling of reservoirs (Poesen and Hooke, 1997; Ben Slimane et al., 2016; Mekonnen et al., 2016). To mitigate the problem of sediments from agricultural areas reaching the channel and, in a later stage, reaching reservoirs and other surface waters, it is important to understand the connectivity between hillslopes, channels and the outlet of the catchment.

Hillslope-channel connectivity depends on hillslope topography, soil types and structure, (riparian) vegetation and management practices (Harvey, 2001; Kirkby et al., 2002). Hillslopes can be directly connected to the channel, with steep slopes, no floodplain and no riparian vegetation or be unconnected through floodplains and dense riparian vegetation. The structure of the hillslope-channel connection (structural connectivity) and the processes that act on that structure (functional connectivity) determine the existence and size of the hillslope-channel connections (Brunsden, 1993; Bracken and Croke, 2007).

Several concepts regarding sediment connectivity have been developed over the past years, some of which focus on hillslope-channel connectivity. One of the most recent concepts of connectivity suggests that small events "liberate" sediments which then concentrate on the lower parts of the hillslopes and channel, gradually increasing sediment connectivity (Bracken et al., 2015). Most of these sediments do not reach the outlet of the catchment during those small events. During a large event, when the previously deposited sediments have caused higher sediment connectivity, these sediments are remobilised. These sediments are subsequently exported from the catchment, causing high sediment discharge at the outlet. This to some extent contrasts with the study of (Cammeraat, 2002), who showed that during small events only small pockets within a catchment are active and have only a very minor (or no) connection to the channels. Large events activate the entire catchment, making sediment transport from the hillslope to the channel and out of the catchment possible. At the end of such an event, large amount of sediments might deposit in the channel, which is then gradually removed by small events. Supporting this model, a study of (Thompson et al., 2016) showed that smaller events are more effective in transporting sediments through a channel, because during large events, large amounts of sediments are deposited on the banks and floodplains. As a consequence, the amount of sediment exported out of the catchment during an event is an indirect result of hillslope-channel connectivity and the continuation of sediment transport within the channel. To determine the functioning of hillslope-channel connectivity, and the continuation of sediment transport within the channel, it is necessary to obtain data on sediment transport from 
the hillslopes to the channels. Furthermore, it is necessary to simultaneously look at factors that influence sediment export out of the catchment.

Sediment tracers can be used to determine which areas on a hillslope contribute sediments to the channel. Tracers have been increasingly used in studies looking at the redistribution of sediments on hillslopes (Guzmán et al., 2013). Rare-Earth oxides (REOs) are types of tracers that are actively applied to the soil. REOs occur naturally in soils in small concentrations, but are applied to the soil at 10-100 times the background concentrations by lawn spreaders or by spraying (Polyakov et al., 2004; Kimoto et al., 2006; Deasy and Quinton, 2010).

Principal component analyses or cluster analyses are often done to analyse factors that influence the hydrological behaviour and the sediment export of a catchment (GarcíaRuiz et al., 2005; Zabaleta et al., 2007; Giménez et al., 2012b). Many of these methods can assess which factors are important for e.g. sediment export at the outlet of a catchment, but they do not always take the interaction between variables into account. Furthermore, they are often not able to take categorical and continuous variables into account side-by-side. Techniques that do take these interactions into account are machine learning techniques. Machine learning techniques are powerful tools that can be used for regression analysis, and moreover, can be used to assess the importance of (categorical) variables and the interaction between variables. One such machine learning algorithm is Random Forest (RF), which has already been successfully applied to improve the mapping of soil characteristics (Hengl et al., 2015) and to model suspended sediment concentrations (Francke et al., 2008). Determining key variables and their interaction for hillslope-channel connectivity has not been done yet using RF.

Hence, the objective of this study was to assess catchment sediment dynamics regarding hillslope-channel connectivity and within-channel sediment transport for a Mediterranean agricultural catchment. We assessed factors influencing hillslopechannel connectivity and resulting catchment sediment yield for varying event magnitudes. The connectivity behaviour of the catchment was compared to connectivity behaviour as described by several conceptual models. To assess hillslopechannel connectivity, transport of sediments from the hillslope to the channel was measured using sediment tracing and influencing factors for catchment connectivity were assessed using the Random Forest regression method.

\subsection{Methods}

\subsubsection{Study Area and data}

The 'Latxaga' catchment $\left(2.07 \mathrm{~km}^{2}\right)$ is located in Northern Spain in the autonomous region of Navarre (Figure 3.1). The climate is humid sub-Mediterranean, with mean annual precipitation of $835 \mathrm{~mm}$, the majority of which falls from October to April (Gobierno de Navarra, 2001). Soils are a silty clay loam, with large, stable aggregates and land use is predominantly agriculture, of which winter wheat is the most abundant crop (Giménez et al., 2012b; Chahor et al., 2014). Slopes in the catchment can be steep, up to $30 \%$, but towards the main channel the slope angles decrease to approximately 5-7\%. Daily hydrological, meteorological and sediment data is available for the period 
2002-2015, for details on collection and devices please see: Casalí et al., $(2008,2010)$, Giménez et al. (2012b), Chahor et al. (2014). Furthermore, a high-resolution $(10 \mathrm{~cm})$ digital terrain model of February 2015 was available for the interpretation of flow paths on the hillslope (Masselink et al., 2017).

\subsubsection{Rare-Earth Oxides tracer application, sampling and interpolation}

Rare-Earth Oxide (REO) tracers were used to assess whether or not sediment from the studied hillslope (Figure 3.1) was transported to the channel during the winter 20142015. The hillslope was selected because of its topography and vegetation arrangement. The hillslope is representative for the area, because like almost all hillslopes in the catchment, it contains a steep area with both agriculture and a seminatural shrub area. It is connected to the channel via a relatively flat area and a densely vegetated riparian zone.

REO tracers strongly adhere to soil particles, without changing the behaviour of these particles and their aggregates (Zhang et al., 2001). Four REO types were used: Erbiumoxide $\left(\mathrm{Er}_{2} \mathrm{O}_{3}\right)$, Yttriumoxide $\left(\mathrm{Y}_{2} \mathrm{O}_{3}\right)$, Praseodymiumoxide $\left(\mathrm{Pr}_{2} \mathrm{O}_{3}\right)$ and Samariumoxide $\left(\mathrm{Sm}_{2} \mathrm{O}_{3}\right)$, later on referred to as $\mathrm{Er}, \mathrm{Y}, \mathrm{Pr}$ and $\mathrm{Sm}$, respectively. Laboratory tests showed that these REO tracers penetrated to a maximum of $1 \mathrm{~cm}$ after a sequence of 3 simulated rain events, which confirmed the limited vertical mobility of the REO tracers within the soils of the study area and confirmed the utility of these tracers for assessing hillslope-channel connections.

To determine background concentrations of the four REOs, 20 samples were taken at different locations on the hillslope before the start of the experiment. These background concentrations were used to calculate the necessary amount of tracer that needed to be applied to reach at least a concentration 10 times that of the background concentration (Polyakov et al., 2004).

REO tracers were mixed with soil from the hillslope, which was dried and ground before mixing. The REO tracers were applied to the hillslope sections on October 302014 , after tillage and seeding of the winter wheat. The REO tracers were applied using a standard lawn spreader that was calibrated to disperse $500 \mathrm{~g}$ of mixture on a stretch of 10 meters on flat, even terrain (Table 3.1). In contrast with earlier studies using REOs spread with a lawn spreader (Polyakov et al., 2004; Kimoto et al., 2006), applied tracers were not mixed into the ground by disking or tillage because: (i) we wanted to keep the oxides within the applied areas, (ii) we did not want to make any changes to normal farming practices, and (iii) the main focus of this study was on the assessment of hillslopechannel connectivity, and, therefore not on calculating exact amounts of displacement of sediments. This choice is supported by Deasy and Quinton (2010), who found that the incorporation of the REO tracers through disking caused high sediment yields for the first events after disking.

Compound samples existing out of 5 randomly taken samples within the application areas were used to determine the final mean application concentrations. The 
concentrations were assumed to be uniformly spread within each of the application areas.
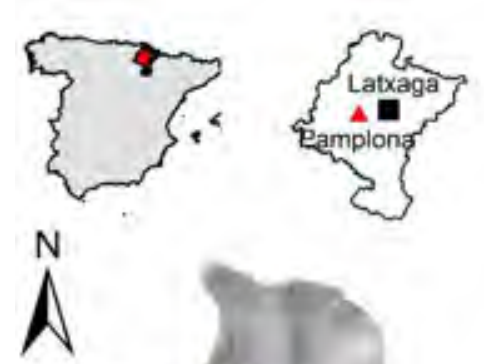

$\AA^{N}$

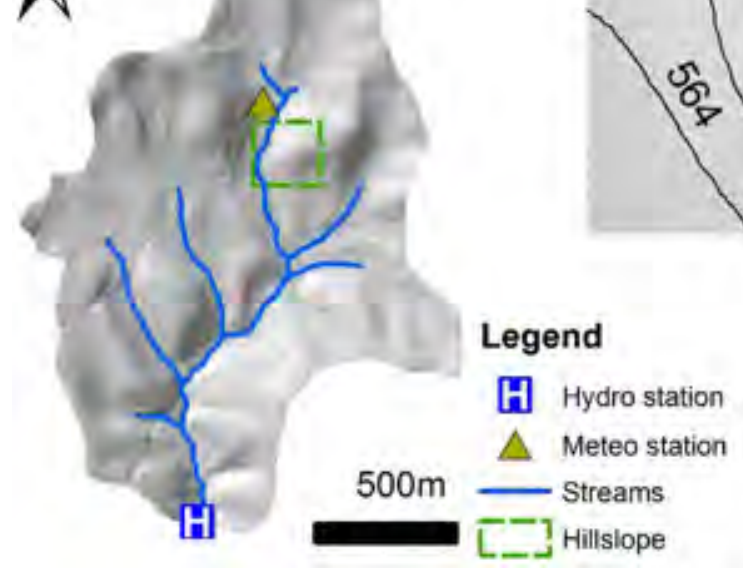

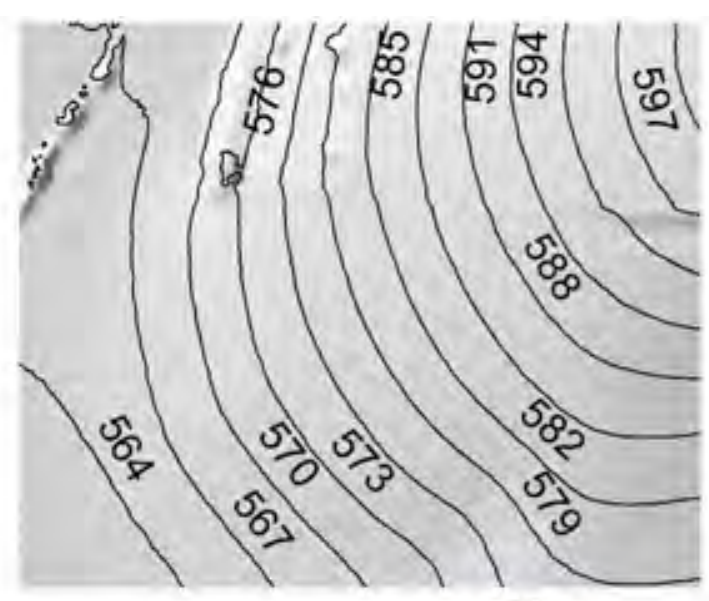

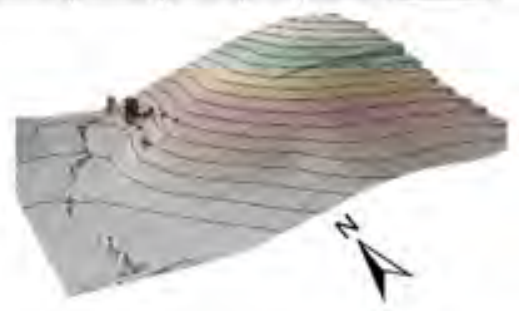

Figure 3.1 The study area of Latxaga, located in Navarre, Spain. The hillslope on which the tracer study was done is depicted on the right in $2 \mathrm{~d}$ (top) and $2.5 \mathrm{~d}$ (bottom). Contour lines depict the altitude of the hillslope.

At the beginning of summer (June 30, 2015), samples were taken for the assessment of sediment movement. This was done just before harvest of the winter wheat to ensure minimum disturbance by machinery. 102 compound samples, consisting of 5 samples taken within a $1 \mathrm{~m}^{2}$ area were taken in a stratified random sampling approach. A set of tractor tracks parallel to the slope was additionally sampled to follow the erosion and sedimentation patterns within the tracks. The tractor tracks were caused by normal farming practices (fertiliser and pesticide application). Furthermore, 8 compound samples were taken of sediments in the channel bed, ranging from next to the hillslope to the outlet of the catchment. These channel samples were taken from both the areas in the thalweg, as well as close to the banks to well represent the area in which the samples were taken. Grain size distributions of the channel sediments were assumed to be similar to the soils within the fields, although they might have been enriched by clay and/or silt.

Samples were dried, sieved to $2 \mathrm{~mm}$ and colloid ground. Subsamples of $500 \mathrm{mg}$ were destructed using an Aqua Regia method. This entails subjecting the sample to $6 \mathrm{~mL} \mathrm{HCL}$ and $2 \mathrm{~mL}$ HNO3 and leaving this standing overnight. Afterwards the samples were heated to $103{ }^{\circ} \mathrm{C}$ for 2 hours. This completely dissolves the oxides. REO concentrations were measured using a high-resolution ICP-MS (Thermo Scientific Element 2). 
Table 3.1 Mean background concentrations, concentrations after application and the amount of times the background concentration was multiplied according to measured concentrations.

\begin{tabular}{lccc}
\hline & $\begin{array}{c}\text { Mean Background Concentration } \\
(\mathrm{mg} \mathrm{kg}-1)\end{array}$ & $\begin{array}{c}\text { Concentration after application } \\
(\mathrm{mg} \mathrm{kg}-1)\end{array}$ & $\begin{array}{c}\text { Times background } \\
\text { concentration }\end{array}$ \\
\hline $\mathrm{Er}$ & 0.96 & 40.9 & 42.78 \\
$\mathrm{Y}$ & 9.41 & 124 & 13.18 \\
$\mathrm{Pr}$ & 2.39 & 370 & 154.52 \\
$\mathrm{Sm}$ & 2.61 & 183 & 70.10 \\
\hline
\end{tabular}

Measured sample concentrations were compared with the normal background concentration range by using a $99 \%$ confidence interval of the measured background values. Samples where REO concentrations were above background concentrations for the confidence interval were interpolated using an adaptation of the standard inverse distance weighting interpolation method. The standard method, unrealistically, does not take into account that REOs can only move downslope. To solve this, the interpolated values were constrained by using flow paths of the high-resolution DTM.

\subsubsection{Random Forests for determining hillslope-channel coupling during large and small events}

\section{Random Forests}

Random Forests (RF) is an ensemble machine learning method for classification or regression problems (Breiman, 2001). RF can deal with large datasets of observations, and also with a large number of predictor variables. $\mathrm{RF}$ is not restricted to normally distributed data, does not assume linear relations, and can incorporate categorical variables. $\mathrm{RF}$ combines an ensemble of models (classification or regression trees) into one prediction model. In contrast to single classification trees, RF does not suffer from overfitting because of the Strong Law of Large Numbers (Feller, 1968). Overfitting refers to the problem that a model works (almost) perfectly on a training set but performs poorly on a test set.

RF has many advantages over other machine learning algorithms: it is not fully a blackbox algorithm, it can calculate the model error internally so there is no need for a training and a validation set and, finally, it determines for each variable the importance for the classification or regression of the target variable. A disadvantage of the method is that for large datasets in combination with a large forest, the operations can become slow and that the model does not perform well for predictions that are outside of the range of training samples.

$\mathrm{RF}$ uses random subsets of measured variables and predictor variables (bootstrapping) to create many decision or regression trees. The final prediction value in the case of regression is the mean predicted value of all trees within the forest (Figure 3.2). 


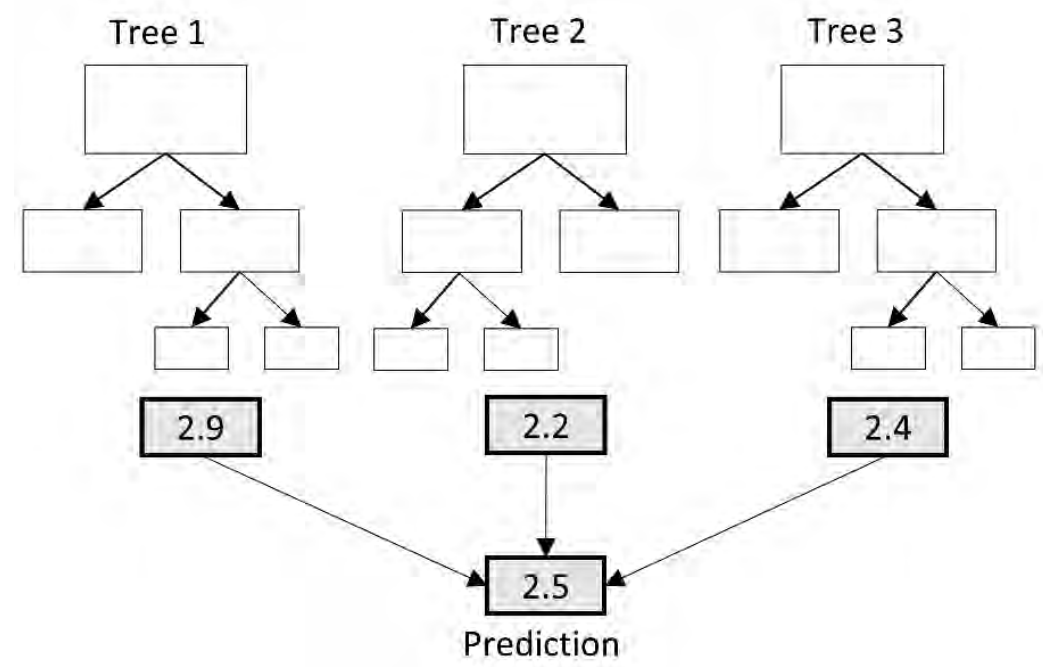

Figure 3.2 Example of a random forest, existing out of three regression trees. The prediction variables are led through all the trees and the final prediction value is the mean of all individual tree outcomes.

\section{Application of Random Forests for determining hillslope-channel connectivity}

The objective of the application of the Random Forest (RF) method was to assess important factors for sediment export at the catchment scale and to determine whether these factors differ between small events and large events. We hypothesized that hillslope-channel connectivity would most likely occur in large events, that, on average, occur a few times per year. Therefore, we chose a threshold for events with an event probability of $5 \%$ or lower, to represent an average of $\sim 18$ days per year with hillslopechannel connections. To determine which events (i.e. days) are within those $5 \%$ percent and at which threshold this occurs, a cumulative distribution function was created, using all daily sediment export data with the ECDF function in the statsmodel.sm library for Python 2.7.

The RF model (RF in Python 2.7 using sklearn.ensemble.RandomForestRegressor) was subsequently run for three datasets: the entire dataset, the dataset for large events ( $<5 \%$ probability) and the dataset for small events ( $25 \%$ probability). All datasets were split into two subsets: a random subset of $70 \%$ for training and $30 \%$ for validation

\section{Input variables and prediction variable}

The total amount of sediment discharge from the catchment $\left(\mathrm{kg} \mathrm{day}^{-1}\right)$ was used as the variable to predict on the basis of daily discharge, meteorological data and derivatives from those data (Table 3.2). Other factors that might play a direct or indirect role for the amount of sediments discharged from the catchment were also taken into account. These factors are day of the year and season, as well as a vegetation index (Normalised Vegetation Index; NDVI), extracted and interpolated at daily basis (Masselink et al., 2016). 
Some of these input variables are collinear up to a certain extent (e.g. cumulative rainfall for 1 and 2 days) but the use of sufficient trees in the random forest ensures that this collinearity does not affect model results (Breiman, 2001). The required number of trees for the forest was determined using the training set from the entire dataset where sediment discharge was larger than $0 \mathrm{~kg}(\mathrm{n}=2451$ days) and the base input variables (Table 3.2). The $\mathrm{R}^{2}$ of variation for the test set was determined for random forests ranging from 1 to 3000 trees using:

$R^{2}=\mathbf{1}-\frac{\sum_{i}\left(y_{i}-f_{i}\right)^{2}}{\sum_{i}\left(y_{i}-\bar{y}\right)^{2}}$

where $\boldsymbol{y}_{\boldsymbol{i}}$ is the measured value at i, $\boldsymbol{f}_{\boldsymbol{i}}$ the predicted value at $\mathrm{i}$, and $\overline{\boldsymbol{y}}$ the mean of the measured values. The threshold at which the model stabilised was chosen as the number of trees necessary in the forest.

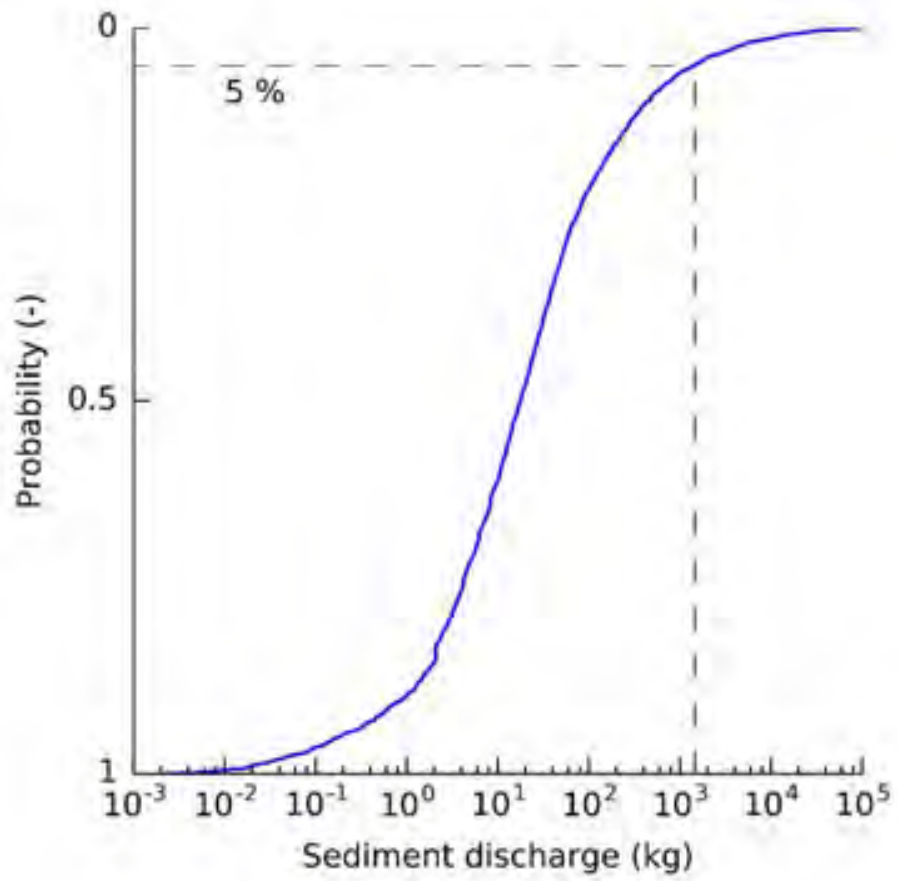

Figure 3.3 Cumulative probability of sediment discharge for a random day, based on $\sim 15$ years of daily sediment discharge measurements. The dotted line indicates the threshold at $5 \%$ and the associated sediment discharge.

\section{Determining Important variables for small and large events}

To determine differences between the behaviour of the catchment during large events and small events, the datasets for both type of models were modelled twice, once using the basic variables and once with additional variables that possibly influence the behaviour within the catchment regarding sediment transport (Table 3.2), either for small or for large events. 
Table 3.2 Basic input variables and additional variables for the datasets containing large and small events for the RF models

\begin{tabular}{|c|c|c|}
\hline Name & Dataset & Description \\
\hline $\mathrm{P}$ & All & Daily precipitation (mm) \\
\hline $\mathrm{P}-1$ & All & Precipitation of day $\mathrm{t}-1(\mathrm{~mm})$ \\
\hline P-2 & All & Precipitation sum of two antecedent days (mm) \\
\hline P-5 & All & Precipitation sum of five antecedent days (mm) \\
\hline P10 & All & Max 10 min Precipitation during the day (mm) \\
\hline P30 & All & Max 30 min Precipitation during the day (mm) \\
\hline P60 & All & Max 60 min Precipitation during the day (mm) \\
\hline P120 & All & Max 120 min Precipitation during the day (mm) \\
\hline day number & All & Day of the year \\
\hline season & All & Season: Winter, Spring, Summer, Autumn \\
\hline NDVI & All & $\begin{array}{l}\text { Daily interpolated Vegetation Index data (period 1984-2014; } \\
\text { see Masselink et al., (2016) }\end{array}$ \\
\hline \multicolumn{3}{|c|}{ Additional Variables } \\
\hline D_Event & Large, Small & Days since last large event \\
\hline Pcum & Large & Sum of precipitation since last event \\
\hline Scum & Large & Sum of sediment discharge since last event \\
\hline Event_S & Small & Size of the last large event $(\mathrm{kg})$ \\
\hline
\end{tabular}

To test whether small events affect sediment export of large events, large events affect sediment export of small events or that the influence is mutual, the influence of several input variables on model performance and variable importance was tested.

In the connectivity concept of (Bracken et al., 2015), small events gradually increase sediment connectivity through depositing sediments near or in the channel, which are then removed during large events. The ratio between the total amount of precipitation and the total amount of sediment discharge could be indicative for the amount of sediment that has accumulated near or in the channel in between large events; many small events sum up to large amounts of precipitation but low sediment export, while few large events might sum up to less precipitation but more sediment export. Furthermore, the amount of time that has passed since the last large event could play a large role in the amount of sediment accumulation in or near the channel. Therefore, the variables ' $\mathrm{P}_{\text {cum', ' ' }} \mathrm{S}_{\mathrm{cum}}$ ' and 'D_Event' were added, which correspond to the sum of precipitation since the last large event, the sum of sediment export since the last event and the time since the last large event.

In the alternative case, where large amounts of sediments are deposited at the end of a large event, the number of days after the event and the magnitude of the large event 
could influence the sediment export of subsequent small events. Therefore, to test if large events influence subsequent small events the input variables ' $D$ _Event' and 'Event_S' were introduced into the RF model, which are the days passed since the last large event and the magnitude of that event.

The number of large events is small compared to the number of small events (i.e. 5\% of number of small events). This might lead to unbalanced results for the model performance, depending on which random subset is taken for training and validation. To be able to compare the results for both conceptual models, the models were trained and validated for 100 different random subsets.

Model performance was tested by calculating the $\mathrm{R}^{2}$ of variation (eq. 3.1) and by calculating the root mean square error (RMSE in $\mathrm{kg} \mathrm{day}^{-1}$ ):

$R M S E=\sqrt{\frac{\sum_{t=1}^{n}\left(\hat{y}_{t}-y_{t}\right)^{2}}{n}}$

where $\hat{\boldsymbol{y}}$ are the predicted values, $\mathrm{y}$ the measured values and $\mathrm{n}$ the number of samples. Model performance ( $\mathrm{R}^{2}$ and RMSE) was calculated for all 100 runs for the RF models and the sample medians between the models with the basic input variables and the additional variables were compared using a non-parametric Mann-Whitney $\mathrm{U}$ test ( $\alpha$ $=0.05$ ).

Furthermore, the variable importance of all input variables was assessed, using the calculated variable importance from the RandomForestRegressor function in the sklearn.ensemble package in Python 2.7. A level of 5\% importance was assumed as a threshold for valuable contribution for the model.

\subsection{Results}

\subsubsection{Hillslope-Channel coupling assessment for 2014-2015 winter using Rare- Earth Oxides}

The interpolated Rare-Earth Oxide (REO) observations show that there has been little sediment transport during winter (Figure 3.4). None of the sediments that had been tagged with tracers reached the channel. The only location where REOs had moved significantly out of the application area was within the tractor tracks, albeit not much farther downslope (28 $\mathrm{m}$ from the sampling area). The samples taken in the channel (Figure 3.4) showed no trace of any of the REOs that were applied on the hillslope.

These results show that during the 2014-2015 winter the number of erosive rainfall events has not been enough to mobilise sediments and to transport these sediments to the foot of the hillslope and into the channel. This is probably related to the maximum precipitation intensities of the winter of 2014-2015, which are lower than those in three previous winters in which more hillslope-channel connectivity was observed (Table 3.3).

The RF model on the entire dataset using only the basic variables starts to stabilise at a number of trees of around 1000 (Figure 3.5). In order to ensure a stable model when 
including more variables, all consecutive model runs were done with 1500 trees in the forest.

The results of the RF model of the total dataset (Figure 3.6) show that the model underperforms $\left(R^{2}=0.01\right)$, which is mainly due to the presence of two outliers. When these outliers are removed the model results explain $45 \%$ of the variation $\left(R^{2}=0.45\right)$. $\mathrm{RF}$ is known to underperform in the case the test data is not within the range of the training data. The variable importance of the total dataset shows that mainly antecedent precipitation, precipitation on the day itself and precipitation intensity control the model, followed by the day of the year (Julian day). The vegetation index (NDVI) and the season are relatively unimportant variables in the model.
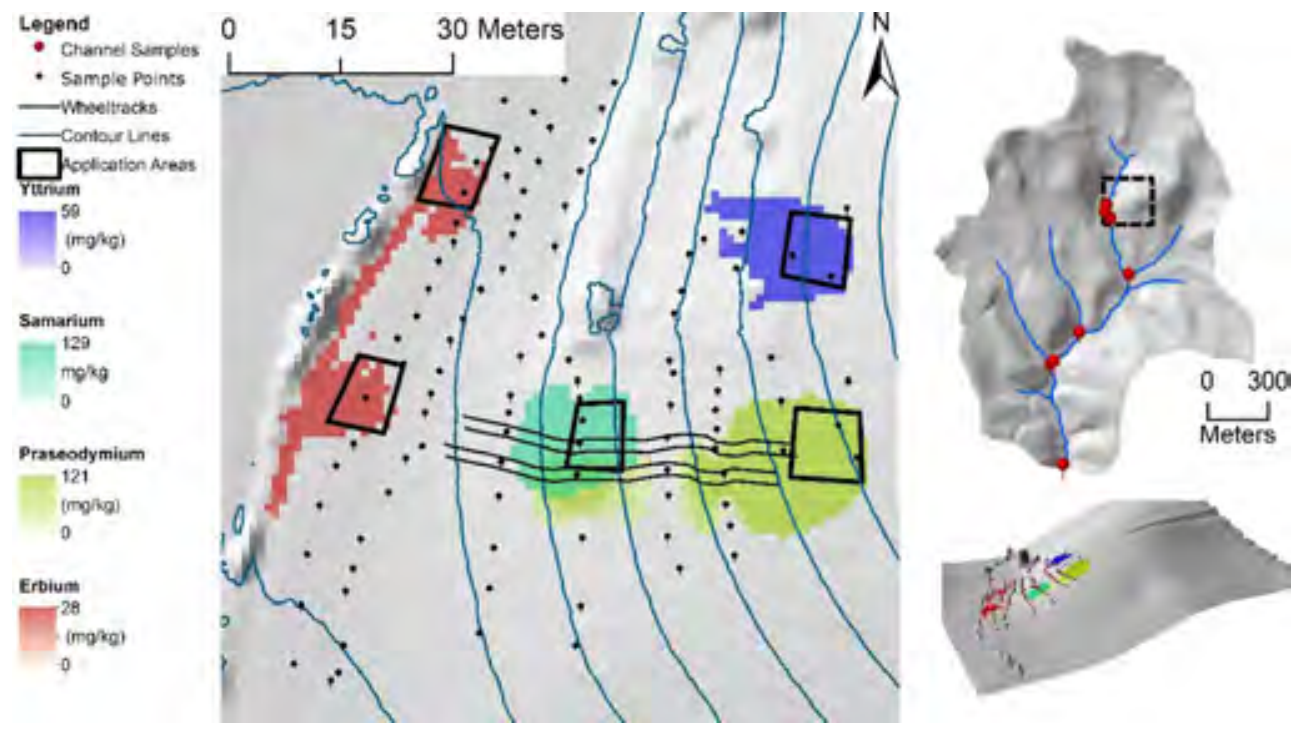

Figure 3.4. Hillslope showing application areas of Rare-Earth Oxides, sample locations and interpolated concentrations. Depicted concentrations are measured concentrations minus the background concentrations. The figure shows contour lines and the location of the tractor wheel tracks. Right side of the figure shows a $2.5 \mathrm{~d}$ representation of the hillslope, showing the concentrations of REOs. The top right figure shows the locations of the hillslope within the catchment and the locations of the samples taken in the channel.

The RF model results with the basic input variables for the large events (Figure 3.6) show that the highest $R^{2}$ still only explains $1 \%$ of the variation $\left(R^{2}=0.01\right)$ and the RMSE is $22568 \mathrm{~kg}$. When including the additional variables, the maximum $\mathrm{R}^{2}$ increases to 0.15 and the RMSE decreases to $20806 \mathrm{~kg}$. The variable importance plots show that for the basic model the most important variables are (antecedent) precipitation and precipitation intensity, followed by the Julian day and the vegetation Index. When the three extra variables ( $\mathrm{P}_{\text {cum, }}, \mathrm{S}_{\text {cum, }} \mathrm{D}_{-}$event) are included, these become the most important variables for modelling sediment discharge. 
Table 3.3. The maximum cumulative amount of precipitation ( $\mathrm{mm}$ ) for 4 consecutive winters (October-June) for the Latxaga catchment for 10 minutes $\left(\mathrm{P}_{10}\right), 30$ minutes $\left(\mathrm{P}_{30}\right), 60$ minutes $\left(\mathrm{P}_{60}\right), 120$ minutes $\left(\mathrm{P}_{120}\right)$, daily, weekly, monthly and total. Total yearly sediment discharge $\left(\mathrm{S}_{\text {total }}\right)$ in kilograms and the number of days with sediment data $(\mathrm{N})$ are also depicted for each year. $\mathrm{P}$ values in between brackets show result of paired t-test to test difference of the means for the variables.

\begin{tabular}{|c|c|c|c|c|c|c|c|c|c|c|}
\hline & P10 & P30 & P60 & P120 & Daily & Weekly & Monthly & Total & Stotal & $\mathrm{N}$ \\
\hline '11-'12 & $\begin{array}{c}6.79 \\
(0.66)\end{array}$ & $\begin{array}{c}9.99 \\
(0.63)\end{array}$ & $\begin{array}{l}17.99 \\
(0.63)\end{array}$ & $\begin{array}{l}19.33 \\
(0.60)\end{array}$ & $\begin{array}{l}43.33 \\
(0.29)\end{array}$ & $\begin{array}{c}71 \\
(0.71)\end{array}$ & $\begin{array}{c}227 \\
(0.80)\end{array}$ & 602 & 18,862 & 214 \\
\hline '12-'13 & $\begin{array}{c}9.12 \\
(0.02)\end{array}$ & $\begin{array}{c}17.4 \\
(<0.001)\end{array}$ & $\begin{array}{c}22.93 \\
(<0.001)\end{array}$ & $\begin{array}{c}27.07 \\
(<0.001)\end{array}$ & $\begin{array}{c}93.09 \\
(0.007)\end{array}$ & $\begin{array}{c}196 \\
(0.03)\end{array}$ & $\begin{array}{c}415 \\
(0.12)\end{array}$ & 1375 & 358,596 & 237 \\
\hline '13-'14 & $\begin{array}{l}12.02 \\
(0.20)\end{array}$ & $\begin{array}{l}19.34 \\
(0.16)\end{array}$ & $\begin{array}{c}22.1 \\
(0.13)\end{array}$ & $\begin{array}{c}22.1 \\
(0.19)\end{array}$ & $\begin{array}{l}40.61 \\
(0.09)\end{array}$ & $\begin{array}{c}118 \\
(0.10)\end{array}$ & $\begin{array}{c}183 \\
(0.19)\end{array}$ & 1043 & 310,728 & 235 \\
\hline '14-'15 & 5.36 & 10.12 & 13.09 & 22.13 & 54.77 & 115 & 193 & 675 & 198,561 & 239 \\
\hline
\end{tabular}

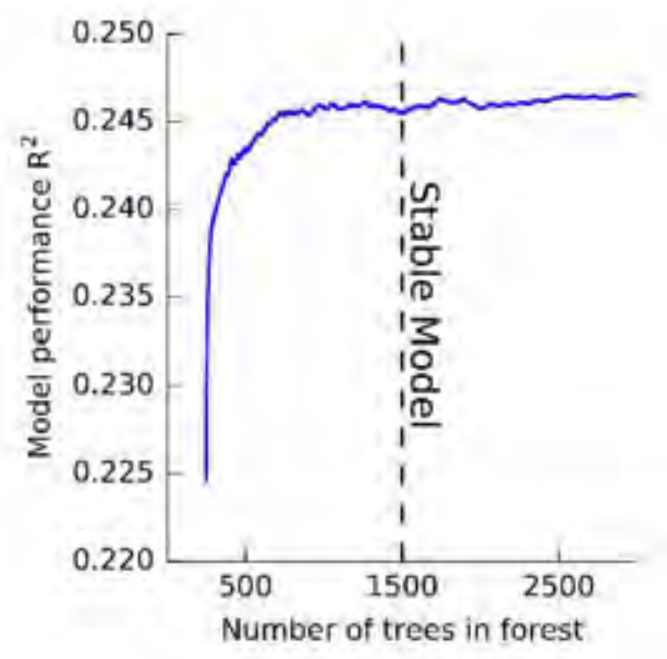

Figure 3.5. Model performance for the number of trees in a forest for the base variables on the entire dataset $(n=2451)$.

The RF model results with the basic input variables for the small events (Figure 3.6) shows a higher $\mathrm{R}^{2}$ value $\left(\mathrm{R}^{2}=0.42\right)$ than the model for the large events. The RMSE value $(138 \mathrm{~kg})$ is also lower than for the large events, but these values cannot be compared, because of the difference of magnitude of events. When including the additional variables, the maximum $\mathrm{R}^{2}$ of the model increases to 0.56 and the RMSE decreases to $109 \mathrm{~kg}$. The variable importance of the models shows that the most important variables for the basic input variables are 5-day antecedent precipitation, the Julian day and the vegetation index (NDVI). The precipitation on the day itself is of much less importance. 
When the three extra variables are included, the days since the last event becomes the most important parameter for the modelling of sediment discharge. The Spearman correlation coefficient between the days since the last event and the sediment output is $-0.55\left(\mathrm{p}<10^{-181}\right)$, showing a negative correlation between the number of days since the event and the sediment output.

The results of the Mann-Whitney $\mathrm{U}$ test show that the differences for the medians for the $\mathrm{R}^{2}$ and the RMSE for the model with base variables and the model with extra variables for the large events are not significantly different ( $\mathrm{p}=0.65$ and $\mathrm{p}=0.76$ resp.). For the small events the medians for the model with base variables and extra variables, the $\mathrm{R}^{2}$ and RMSE are significantly different ( $\mathrm{p}<1 \mathrm{e}-15$ for both). This means there is no significant improvement from adding the additional variables for the large events, whereas there is a significant improvement when adding the additional variables for the small events.

\subsection{Discussion}

\subsubsection{Factors controlling Hillslope-Channel connectivity}

Using the Rare-Earth Oxide (REO) tracers we were able to demonstrate that hillslopechannel connectivity was low for the winter of 2014-2015, with low precipitationintensity events (Figure 3.4). This means that these events contributed little to no sediment to the channel. The only place where some more sediment transport took place was in the tractor tracks parallel to the slope. Even though the movement of sediments was still limited $(<30 \mathrm{~m})$, this shows the importance of linear features like tractor tracks, rills and drainage ditches for hillslope-channel connectivity (Basher and Ross, 2001; Heathwaite et al., 2005; Collins and Davison, 2009).

Even on days that have large total precipitation (i.e. $54.77 \mathrm{~mm}$ ), precipitation intensity seems to be the controlling factor for sediment mobilisation and, therefore, for hillslope-channel connectivity. The p-values of the independent t-tests show that there is a large difference for precipitation intensities, especially between the winter of 20122013 and the winter in which the REOs were applied (2014-2015; Table 3.3). Precipitation intensity was also found to be the major determinant for hillslope-channel coupling in a modelling study done by (Michaelides and Wainwright, 2002). The REO findings on the studied slope, together with the fact that the slope is representative for other hillslopes within the catchment, indicate that the majority of the sediments leaving the catchment in the winter of 2014-2015 must have come from sources within the channel or the channel banks.

The results of the Random Forest (RF) method for the entire dataset and the large events partly agree with the findings of the REO tracers, in the sense that precipitation intensities are considered to be important variables for estimating sediment discharge. However, antecedent precipitation is considered to be more important than precipitation intensity (Figure 3.6). It is likely that the combination of large amounts of antecedent precipitation and high precipitation intensities lead to a large amount of sediment detachment and enough overland flow to transport these sediments, as also 
Total Dataset $(n=2451)$

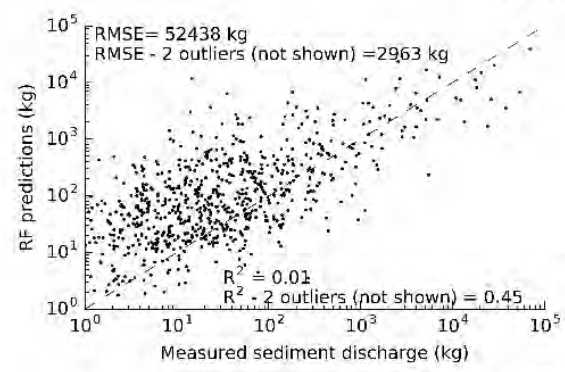

$\begin{array}{lllllllll}0.00 & 0.02 & 0.04 & 0.06 & 0.08 & 0.10 & 0.12 & 0.14 & 0.16\end{array}$
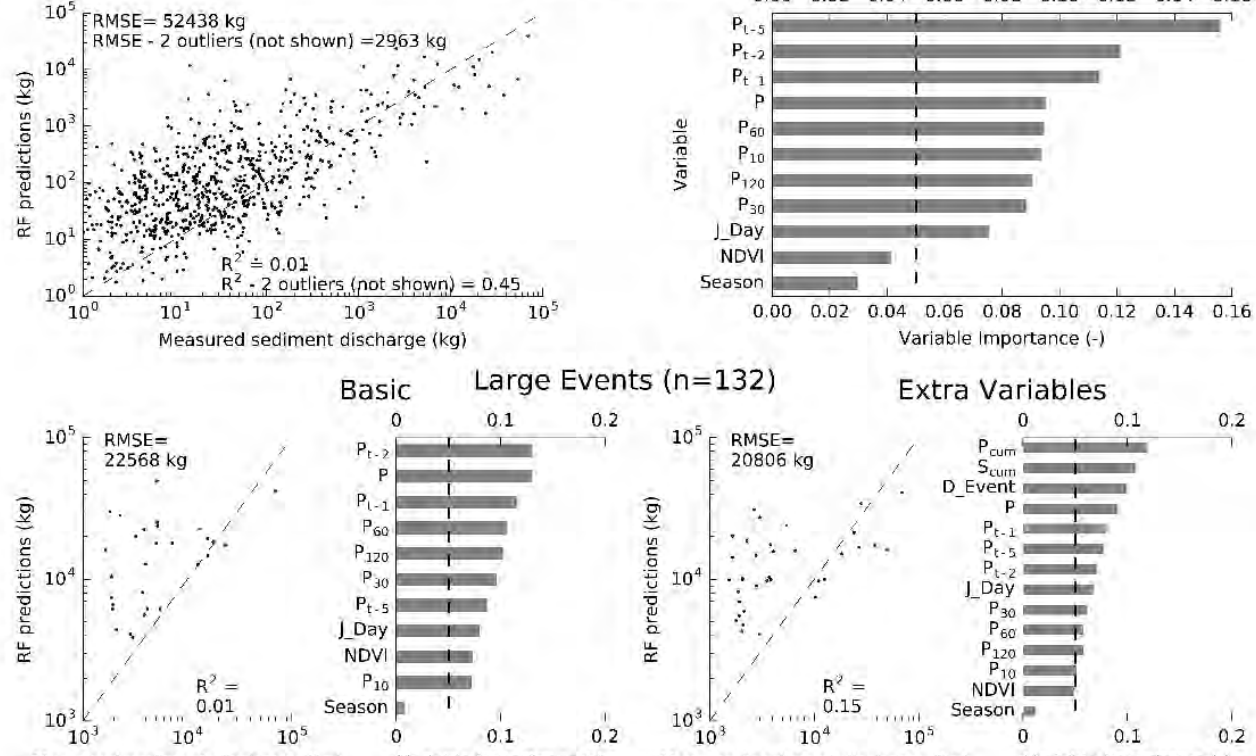

Measured sediment discharge (kg) Variable Importance (-) Measured sediment discharge (kg) Variable Importance (-)
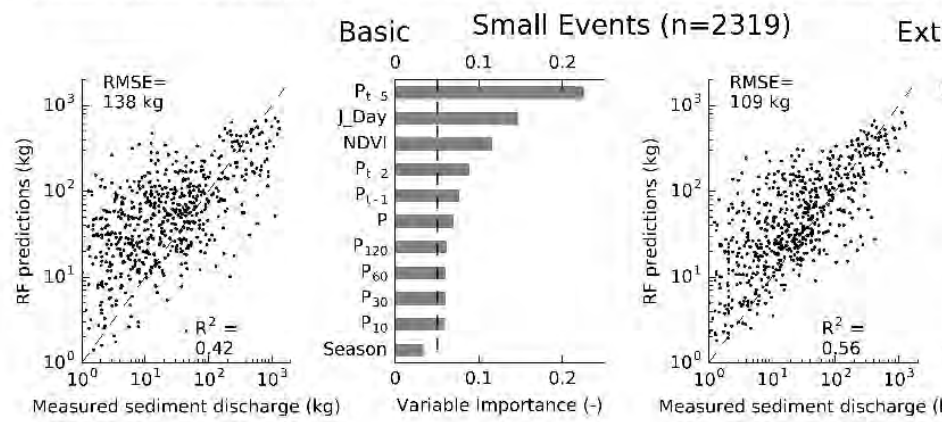

Extra Variables

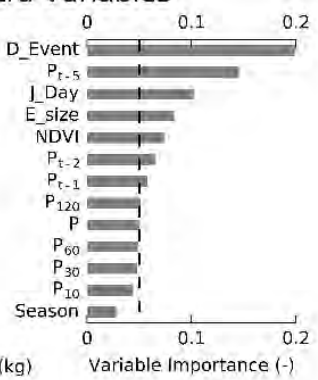

Figure 3.6. Results of the random forest models for the entire dataset (top), the large events (middle) and the small events (bottom). The scatter plots show the measured and predicted sediment discharge quantities ( $\mathrm{kg}$ ) of the best performing model out of 100 model runs and the bar plots show the mean variable importance of all 100 model runs. The left side of the figure for the large and small events show the model runs with the base variables and the right side with the added extra variables. The variable importance bar plots show the $5 \%$ limit at which variables were considered to contribute significantly to the model results.

shown in other studies in Spain (Cantón et al., 2001; Gómez-Plaza et al., 2001; Baartman et al., 2012b; Giménez et al., 2012b). (Giménez et al., 2012b) furthermore, argued that most of the sediments at the outlet of the Latxaga catchment seemed to have come from areas close to the drainage network, which agrees with our findings. Similarly, (Casalí et al., 2008, 2010), suggested that, within the Latxaga catchment, summer storms provoke detachment and sediment movement due to highly erosive events. These sediments, however, do not make it to the outlet of the catchment, due to a lack of overland flow for sediment transport. 
The RF model for the entire dataset and the RF model with the basic variables for large events showed that the factors determining sediment discharge were antecedent precipitation, total daily precipitation and precipitation intensity. Model results for these large events, however, were unsatisfactory (median $\mathrm{R}^{2}<0$ ) and, therefore, little can be said for the actual importance of any of these variables. The unsatisfactory results for the large events most likely originate from i) the use of fewer events for training and validation than for the small events and ii) the low correlation between any of the input variables and the sediment discharge. This means that some of the important variables that determine the amount of sediment discharge are missing from the RF model.

The RF model with the basic variables for small events showed that the factors determining sediment discharge were antecedent precipitation, vegetation cover and the Julian day (day of the year). This shows that seasonality plays a large role in the amount of sediments discharged out of the catchment during small events. In summer, more vegetation is present along and within the channel, retaining more sediments, while in winter this sediment retention is reduced. The variable 'season' was deemed less important, possibly because of its coarse temporal resolution (i.e. only 4 seasons), while the Julian day variable includes more variability over the year (i.e. 365 days). In another study looking at modelling sediment concentrations in the Spanish Pyrenees using a Random Forest model, the Julian day was also one of the most important factors in some catchments (Francke et al., 2008).

\subsubsection{Conceptual models for hillslope-channel connectivity}

The extra variables in the RF model did not significantly improve model results for the large events. Therefore, it is not possible with our dataset to assess a possible influence of small events on the sediment discharge of large events through the RF modelling procedure. However, there could be contribution of sediments from small events to the areas near the channel, but the signal of this sediment accumulation is relatively minimal compared to the large amounts of sediments mobilised during large events.

Model efficiency for the RF models for small events significantly increases when including the additional variables ( $\mathrm{p}<1 \mathrm{e}-15)$, with a negative Spearman correlation of $0.55\left(\mathrm{p}<10^{-181}\right)$. This shows that the amount of time that has passed since a large event and the size of that event have a large influence on sediment export of days that follow with little or no rainfall. These modelling results, in combination with the sediment tracing experiments indicate that large events not only export large amounts of sediment out of the catchment, but also provide the sediments for small subsequent events that export the sediment out of the catchment. This indicates a similar functioning of a catchment as shown by (Cammeraat, 2002). Furthermore, this shows that at least for Mediterranean settings like the Latxaga catchment, the conceptual model of (Bracken et al., 2015) does not adequately describe the catchment sediment dynamics (Figure 3.7).

The actual sediment dynamics in the Latxaga catchment and many other Mediterranean catchments are more complex than either two conceptual models. In reality, a 
combination of the two is more likely, depending on what antecedent conditions are present in terms of e.g. vegetation and soil moisture. The results show that there is feedback of large events on small events and perhaps also vice versa. However, this hypothesis needs to be further validated using field data.

Field data that need to be gathered to test the hypothesis are sediment tracing data either after every event, and/or tracking sediments over multiple seasons and years (Kimoto et al., 2006). In addition, sediment volumes within the channel need to be quantified to obtain a closing sediment budget.

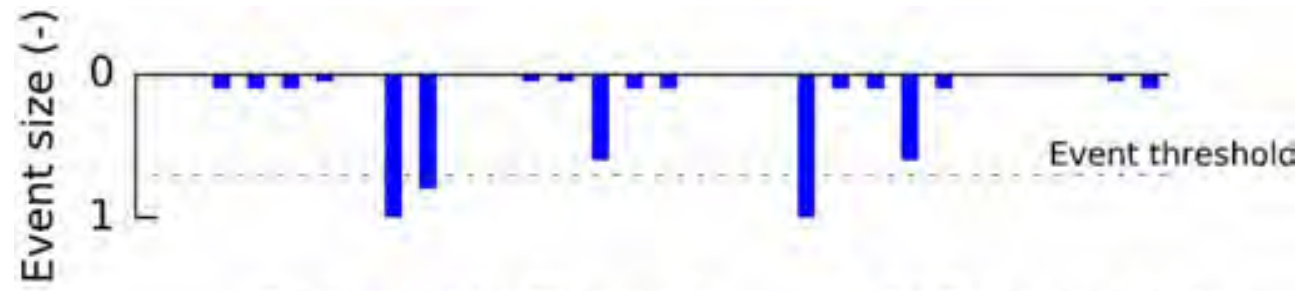

\section{- Sediment in Channel H-C connectivity}

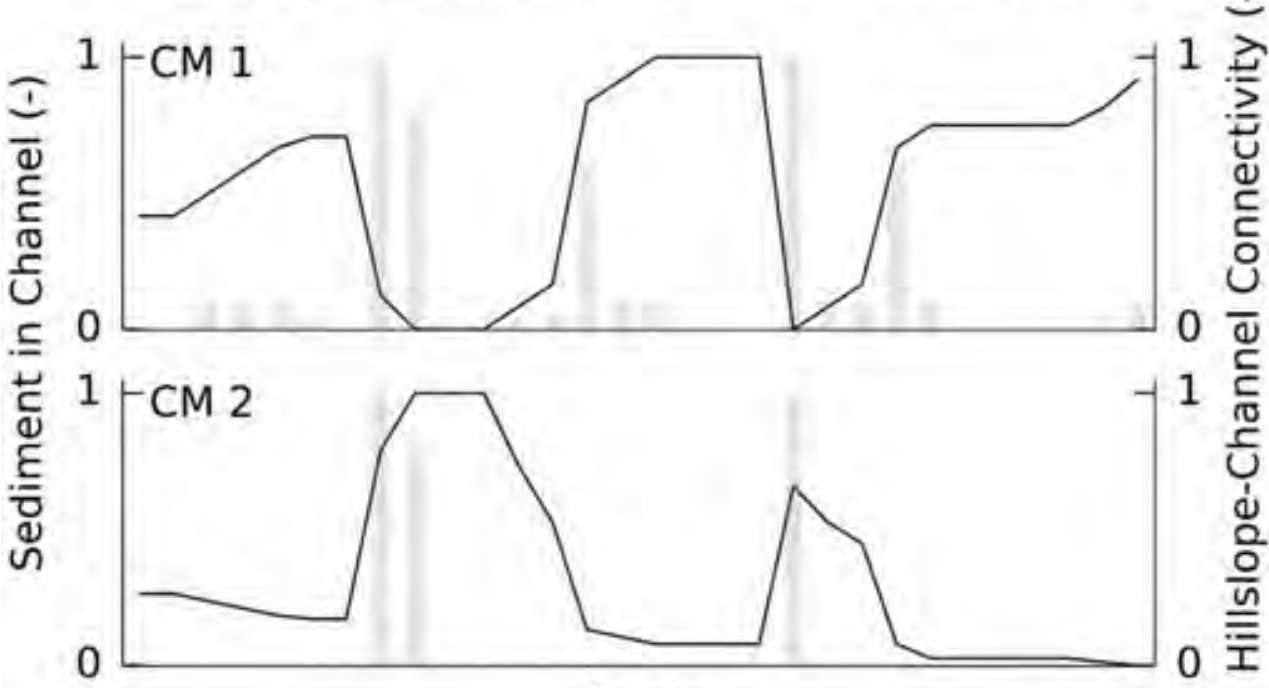

Time

Figure 3.7. Illustration of catchment response for two different Conceptual Models (CM). The top plot shows a sequence of events, with an arbitrary "event threshold" of connectivity depicted. CM1 shows the conceptual model as proposed by Bracken et al. (2015), with sediments from the hillslopes gradually accumulating in and near the channel. These sediments are flushed out of those areas during large events, with which the system "resets". CM2 shows the model as measured by Cammeraat (2002) and shown in this study. Sediments in and near the channel are gradually removed and are replenished during large, fully connecting events.

\subsection{Conclusion}

In this study, we looked at hillslope-channel connectivity, factors influencing sediment connectivity and sediment export out of the agricultural Latxaga catchment in Navarre, Spain. For measuring hillslope-channel sediment transport, Rare-Earth Oxide (REO) 
tracers were applied to a hillslope preceding the winter of 2014-2015. The results showed that during the winter there have been no sediments transported from the hillslope into the channel, which was most likely due to low precipitation intensities. The sediment connectivity of the catchment was assessed using a Random Forest (RF) machine learning method, which was applied to the entire dataset ( $N=2451$ days) and two subsets of the whole dataset: small events ( $\mathrm{N}=2319$ days) days and large events ( $\mathrm{N}=132$ days). The model for small events showed that there is a significant increase in model performance when variables related to preceding large events are included $(p<1 e-15)$. Furthermore, the variables related to these large preceding events are the most important variables in the model for prediction of sediment export. The model for large events underperformed and we can, therefore, not draw any immediate conclusions from the model results regarding variable importance. Because we cannot make any conclusions regarding variable importance, we are not certain that small events influence the amount of sediment exported during large events. The large variability in sediment export for large events and the relatively small contribution during large events of sediments earlier mobilised during small events are the most likely cause of the underperformance of the RF models. The sediment dynamics in Latxaga are dominated by sediment mobilisation during large events. These sediments are for a large part exported during those large events. Large amounts of sediments in and near the channel are deposited at the end of the large events, which are gradually removed by subsequent small events. To determine how exactly large and small events influence each other, we need to gather more sediment tracer data on hillslope-channel connectivity and within-channel sediment dynamics. 


\section{Acknowledgements}

This study was funded by the Dutch organisation for scientific research (NWO), grant number 822.01.004, the Spanish Ministry of Science and Innovation, project CGL201564284-C2-1-R (MINECO/FEDER) and COST action ES1306. We would like to thank Jokin del Valle de Lersundi of the Department of Rural Development and Environment of the Government of Navarre (Spain) for help in obtaining the sediment data. Furthermore, we are grateful for the help in the field from Paul Ollobarren del Barrio. Lastly we would like to thank Francesc Gallart and an anonymous reviewer for their useful comments on the manuscript. 


\begin{abstract}
Knowledge about connectivity and what affects it, through space and time is needed for taking appropriate action at the right place and/or time by stakeholders. Various conceptual frameworks for hydrological and sediment connectivity have been developed in recent years. For most of these frameworks, the objective was to conceptualise connectivity, not necessarily to infer it from measurements. Studies focussing on measurements of connectivity have so far not been done often. Because of lack of data on connectivity, few real-world data have been used in recent connectivity modelling studies. The aim of this study was to demonstrate that existing data can be used to assess governing factors of connectivity, and how these change over time. Data from three catchments in Navarre, Northern Spain, were used to assess factors that influence hydrologic and sediment connectivity. These factors were used as components in a linear model for discharge and suspended-sediment yield. Three components of connectivity were distinguished: topographical, biological and soil. Changes in the topographical component for the studied periods were considered relatively small, and, therefore, kept constant. Changes in the biological component were determined using the Normalised Difference Vegetation Index. Changes in the soil component were assessed using an Antecedent Precipitation Index. Nash-Sutcliffe model efficiency coefficients were between 0.49 through 0.62 for the discharge models and between 0.23 through 0.3 for the sediment-yield models. We recommend applying the model at smaller spatial scales than catchment scale to minimize the lumping of spatial variability in the components.
\end{abstract}

Published as: Masselink RJH, Keesstra SD, Temme AJAM, Seeger M, Giménez R, Casalí J. 2016. Modelling Discharge and Sediment Yield at Catchment Scale Using Connectivity Components. Land Degradation \& Development 27 (4): 933-945. 


\section{Chapter 4. Modelling discharge and sediment yield at catchment scale using connectivity components}

\subsection{Introduction}

Connectivity is defined as the physical coupling (linkages) of different elements within a landscape regarding (sub)surface flows and associated substances, e.g. sediments and nutrients (Pringle, 2003; Stieglitz et al., 2003; Croke et al., 2005). The concept of connectivity addresses the spatial and temporal variability in runoff, sediment transport and associated substances such as pollutants and how these move through the catchment. Hydrological connectivity describes variability of flow of water on a hillslope or within a catchment; for surface water, soil water or ground water. Sediment connectivity explains which sediment sources contribute and where (semi-) permanent sinks and pathways of sediment are (Bracken and Croke, 2007). Sediment connectivity is, therefore, an important determinant for the total quantity of sediment reaching a catchment outlet. Sediment and associated substances such as herbicides and nutrients that leave the catchment can have negative downstream effects, e.g. eutrophication or siltation of waterways. Knowledge about connectivity and what affects it through space and time is, therefore, invaluable for taking appropriate action at the right place, and/or time by e.g. land managers and farmers (Roehl, 1962; Khanbilvardi and Rogowski, 1984; Croke et al., 2005; Bochet, 2015).

The number of publications in hydrology and geomorphology that include the term connectivity has risen over the past decade (Parsons et al., 2015). Also, many publications in the past have already included connectivity in some way, but might not have used the term connectivity (Dunne and Black, 1970; Cerdà and Doerr, 2010). Among the numerous studies on hydrological and sediment connectivity, the majority have focussed on conceptual frameworks, indices or modelling. The conceptual frameworks and models describe various perspectives on of connectivity (Bracken and Croke, 2007; Turnbull et al., 2008; Lexartza-Artza and Wainwright, 2009; Fryirs, 2012; Bracken et al., 2013, 2015; Heckmann and Schwanghart, 2013). Indices and metrics used to describe connectivity are based on topography using Digital Elevation Models (Cavalli et al., 2013), soil moisture (Ali and Roy, 2010) or a combination of DEM based and field based studies (Borselli et al., 2008). Modelling studies on connectivity have been mainly conceptual, empirical or exploratory and most did not use real world data for validation (e.g. (Meerkerk et al., 2009; Gumiere et al., 2011; Baartman et al., 2013; Kirkby, 2014). An exception to this is the study done by Vigiak et al. (2012), who successfully used an index of connectivity (Borselli et al., 2008) for modelling hillslope sediment delivery ratio. The reason that only few modelling studies have used real world data might be that relevant data are not available for many of the objectives of these studies. The lack of modelling studies validated with real world data present a clear research need.

Among the few studies that do use real world data, most assume that variation in measured hydrological or sediment outflow is a result of varying connectivity under different conditions, but do not infer or quantify connectivity (Cammeraat, 2002). Only 
few studies attempted to infer connectivity from direct measurements, e.g. Ali \& Roy (2010), who used a network of soil moisture sensors to assess 2D and 3D connectivity patterns in soil moisture and how they influence runoff. (James and Roulet, 2007) measured antecedent moisture conditions and found a highly non-linear response of runoff for various rainfall events. There is, however, not necessarily always the need to gather data in new ways specifically aimed at measuring connectivity, because connectivity might be inferred from data which are already available. Many large datasets with data on hydrology, meteorology and sediment transport already exist and we believe that these can be of great use for inferring connectivity on a range of temporal and spatial scales.

The aims of this study were to assess to what extent existing 'traditionally gathered' hydrological and sediment data can be used to assess catchment scale connectivity. We modelled catchment scale discharge and suspended-sediment yield by incorporating a representation of hydrological and sediment connectivity in linear hydrological and sediment-yield models. The models were based on precipitation input and three subdivided components of connectivity: a topographical, soil and biological component. The models were developed with daily discharge and sediment data (13 years) from three catchments with varying sizes and land use in Northern Spain.

\subsection{Methods}

\subsubsection{Study areas and data}

Data from three study areas in Navarre, Northern Spain were used for this study (Figure 4.1). The catchments were set up as research catchments by the government of Navarre to assess erosion in the province within agricultural and semi-natural catchments (Casalí et al., 2010). The catchments are part of a larger network of experimental catchments within the autonomous region of Navarre. The reason these three catchments were chosen was because of data availability and noticeable differences between the three catchments which made it possible to assess different environments.

The Latxaga $\left(2.07 \mathrm{~km}^{2}\right)$ and La Tejeria $\left(1.69 \mathrm{~km}^{2}\right)$ catchments are agricultural catchments, that completely consist of agricultural fields, apart from some shrub areas which are located where agricultural practices are impossible due to too steep slopes or bedrock close to the surface. The majority of crops are winter wheat (Triticum aestivum), barley (Hordeum vulgare) and occasionally legumes (Vicia faba L. and Pisum sativum L.) or sunflower (Helianthus annuus L.)(Table 4.1). The Oskotz forest $\left(5.05 \mathrm{~km}^{2}\right)$ catchment is larger than the two agricultural catchments, and was chosen in order to model the connectivity in catchments with different size and land use. The land cover in Oskotz is mainly forest (89\%), however, some agricultural fields are present (11\%). Climate in the two agricultural catchments is similar with 750-850 mm of precipitation per year (Gobierno de Navarra, 2001), most of which falls in winter, although some convective storms occur during summer. The precipitation pattern is similar in Oskotz, but due to its location closer to the coast and the Pyrenees, precipitation averages to $1242 \mathrm{~mm}$ per year (Gobierno de Navarra, 2001). 

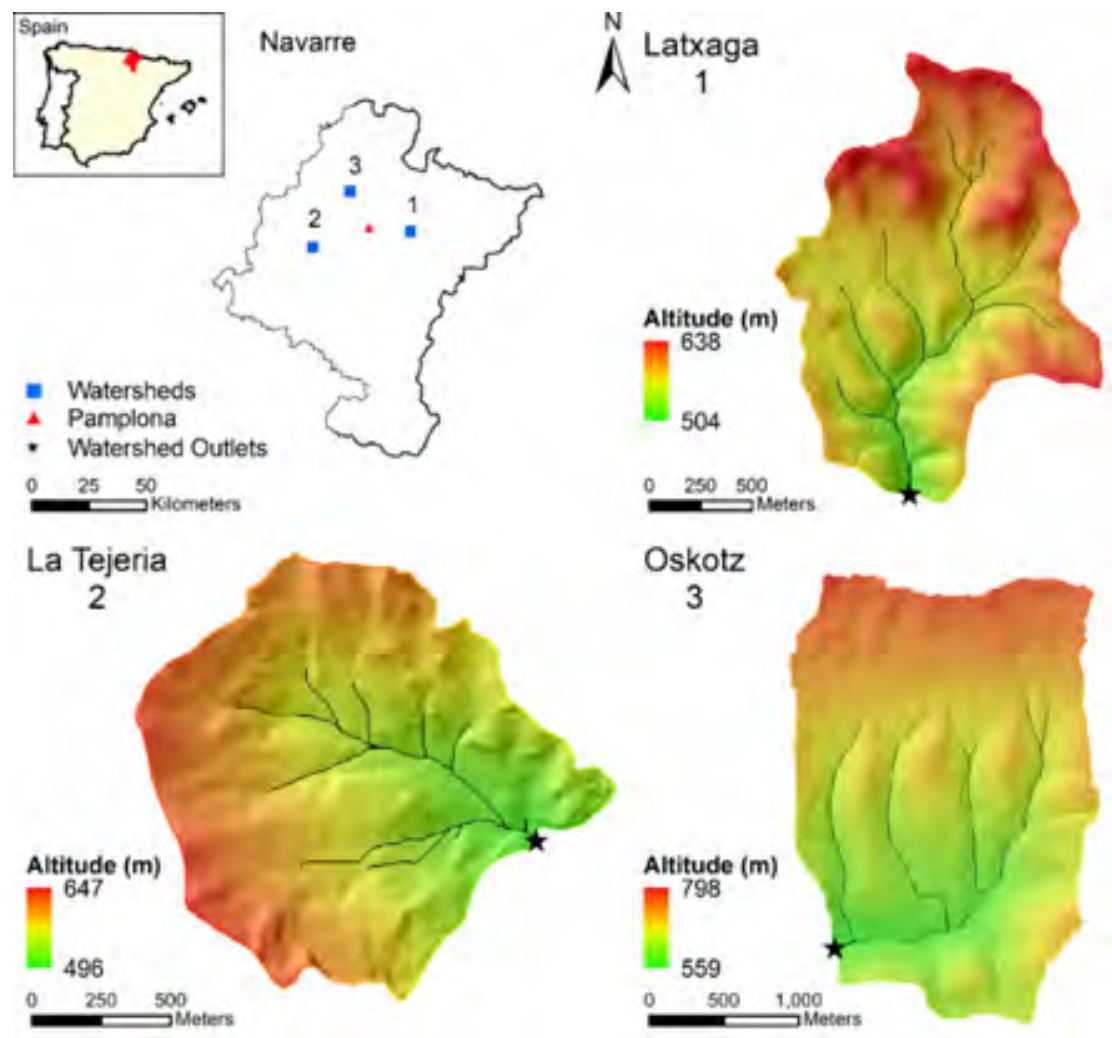

Figure 4.1 Three study areas, located in Navarre, Spain. The principal river networks are indicated with the black lines, and black stars indicate watershed outlets.

Latxaga and La Tejeria are mainly underlain by marls and within La Tejeria some sandstone is also present. The geology in Oskotz is characterised by an alternation of marls and sandy limestone (Gobierno de Navarra, 1997; Casalí et al., 2010). Soil depths in Latxaga average to $1.0 \mathrm{~m}$ and in La Tejeria average to $0.8 \mathrm{~m}$. Organic matter content in both agricultural catchments is around 1-2.5\% (Casalí et al., 2008). Soil depths in Oskotz are more than 1 meter, except for those on eroded hillslopes, which are about 0.5-1 m deep. Organic matter content in Oskotz ranges from 3-6\% (Casalí et al., 2010).

Meteorological and hydrological data for all catchments have been available since 2002 (Table B1). In addition to the catchment specific hydrological and meteorological data there is freely available satellite imagery from the Landsat programme available since 1984 (US Geological Survey), which was used to determine changes in connectivity through changes in vegetation. 
Table 4.1 Characteristics of the study areas (Casalí et al., 2010; Giménez et al., 2012b)

\begin{tabular}{lccc}
\hline & Latxaga & La Tejeria & Oskotz \\
\hline Size (km2) & 2.07 & 1.69 & 5.05 \\
Length of main channel (km) & 2.09 & 1.2 & 2.59 \\
Average slope (\%) & 19.3 & 14.4 & 19.8 \\
Average channel slope (\%) & 4.7 & 8.3 & 5.1 \\
Main land cover & Cereals & Cereals & Forest (89\%)/pasture (11\%) \\
Average precipitation (mm/year) & 876 & 757 & 1,242 \\
\hline
\end{tabular}

\subsubsection{Catchment scale connectivity}

To facilitate the use of existing data, connectivity as a whole in this study was subdivided into three components that might individually connect easily to existing measurements: topographical, biological and soil. These components were chosen because they appear to cover most variables that affect connectivity and can be measured quantitatively. For the purpose of explaining these components in the remainder of this paper, we first define catchment-scale connectivity as the ability of a catchment to transfer runoff and/or detached sediments to the outlet. This is then defined as a function of the three components

$\mathrm{C}=f\left(C_{T}, C_{S}, C_{B},\right)$

where $\mathrm{C}$ is the total catchment connectivity and $\mathrm{C}_{\mathrm{T}}$ the topographical connectivity component, $\mathrm{C}_{\mathrm{S}}$ the soil connectivity component and $\mathrm{C}_{\mathrm{B}}$ the biological connectivity component.

The three individual components of connectivity will be discussed separately and we will then continue how these components were used with the data available for the three catchments in Northern Spain. Linear regression models were created for predicting discharge and suspended sediment yield on a daily basis at the outlet of the catchments.

\section{Topographical connectivity component}

The topographical connectivity component consists of the entire structure of a catchment and the spatial configuration of landforms within the catchment. Next to e.g. configuration of landforms also characteristics like catchment size and shape are important for the topographical connectivity component (Baartman et al., 2013). Topography is one of the main controlling factors in the displacement of sediment and the arrangement of the topography, therefore, plays an important role in the relative amount of sediment reaching the outlet of the catchment (Yuan et al., 2015). The importance of topography is reflected in e.g. the Universal Soil Loss Equation and associated approaches, that all include slope gradient and slope length as one of the determinants (Wischmeier and Smith, 1978). Also all landscape evolution models track erosion, transport and deposition of sediment as a function of topography, with 
different degrees of complexity (Tucker and Hancock, 2010; Temme et al., 2013; Keesstra et al., 2014b).

Compared to soil or vegetation, changes in topography are typically relatively slow. An exception could be change due to extreme (precipitation) events or human influences on the system, such as the building or removing of agricultural terraces (Foster et al., 2012). Changes in topography and landforms can either increase or decrease connectivity within a catchment. Steep or long slopes that are directly coupled to the river system, rills and gullies can all increase connectivity. Decoupling of slopes from the river system by floodplains or river terraces decreases connectivity.

Topographical connectivity can be inferred from digital terrain models (DTMs), aerial photography or (traditional) geomorphological mapping. Currently, techniques exist to create DTMs with resolutions of up to a few centimetres or even millimetres (Westoby et al., 2012; Kaiser et al., 2015; Nadal-Romero et al., 2015). With such DTMs the structure of the landscape can be captured from small scale features like soil roughness up to large scale landforms like floodplains and alluvial fans. With these high-resolution data the topographical component of connectivity can be assessed by the use of e.g. indices, for which much work already has been done (e.g. Borselli et al., 2008; Cavalli et al., 2013).

\section{Soil connectivity component}

The soil connectivity component encompasses all characteristics of the soil that affect hydrological processes and, therefore, sediment transport processes (in absence of gravitational processes; cf. Bracken et al., 2015). These characteristics include e.g. soil texture, structure, moisture, roughness, water repellency and the altering of the soil by burrowing fauna such as earthworms, moles and gophers. The soil connectivity component influences the connectivity at both small and large spatial and temporal scales.

Important properties of the soil connectivity component are grain size distribution and organic matter content, because these influence properties like cohesion, soil water repellency and crusting. Macropores and their connectivity, either formed naturally in the soil or created by burrowing fauna can have an important influence on the amount of water that is transported through the subsurface and, therefore, on the connectivity of water and sediments at the surface (Beven and Germann, 1982; Spence, 2010). Many studies have shown the importance of antecedent soil moisture conditions for the generation of overland flow (Western et al., 2001; Seeger et al., 2004; Butzen et al., 2011). The soil often needs to be close to saturation in humid environments before generating overland flow because infiltration rates are almost always higher than precipitation intensity (Hewlett and Hibbert, 1967; Dunne and Black, 1970). In semiarid regions, precipitation intensity is often higher than infiltration capacity, increasing connectivity during an event through connected flow paths (Cerdà et al., 2016). This rule masks large variation, however; even in catchments with similar soil characteristics the thresholds of overland flow can differ (Godsey et al., 2004; Scherrer et al., 2007). Low soil moisture content in semi-arid regions can lead to soil water 
repellency (Stoof et al., 2011) and soil crusting/sealing (Le Bissonnais et al., 1995), reducing infiltration and in turn, increasing connectivity.

\section{Biological connectivity component}

The biological connectivity component mainly involves effects of vegetation on runoff and sediment transport. Vegetation usually acts as a buffer for sediments, and especially its spatial distribution can be an important factor in the connectivity of a system (Cerdà, 1997; Bautista et al., 2007; Mayor et al., 2008; Marchamalo et al., 2015; Ola et al., 2015). Biological connectivity is typically more dynamic over shorter timescales than the topographical component. Vegetation not only changes over a timescale of several years for instance as forests mature, but considerable changes occur even within a year when e.g. agricultural crops are sown, grow and harvested.

Vegetation affects connectivity in several ways: as cover crops that reduce erosion (Kaspar et al., 2001b; Mekonnen et al., 2015b; Keesstra et al., 2016; Prosdocimi et al., 2016a, 2016b), as buffering riparian vegetation (Clary and Leininger, 2000; Hook, 2003; Verstraeten et al., 2006; Keesstra et al., 2012) and from effects of deforestation, afforestation and forest fires on sediment production and transport (Vanacker et al., 2005; Keesstra, 2007; Sadeghi et al., 2008; Keesstra et al., 2009a, 2009c; Romero-Diaz et al., 2010; Estrany et al., 2015).

\subsubsection{Applying Connectivity components to the study areas}

To assess how well the three components can combine into a model for connectivity, measures for the three components of catchment scale connectivity are needed, as well as a proxy for determining the total catchment connectivity. As noted earlier, the catchment scale connectivity determines which portion of the precipitation and detached sediment reaches the outlet of the catchment and when it reaches the outlet of the catchment. Discharge and the measured sediment yield can, therefore, act as a proxy for determining the catchment scale connectivity in two different linear regression models: one for hydrological connectivity and one for sediment connectivity. The linear regression models were created using the connectivity components to predict discharge and suspended-sediment yield on a daily basis. The effect of the individual components on discharge and sediment yield was then assessed, as well as connectivity changes through time.

\section{Topographical connectivity}

Although the (micro-) topography of the catchments changes constantly throughout the year and the study period, we assumed that these relative small-scale changes over the entire catchment are relatively small compared to the larger topographical structure. The topographical component was, therefore, assumed to be constant over time in all three catchments. Within the models this meant that the topographic component was kept constant, and, therefore, not a parameter in the model. A constant topographical component automatically means that the model results between catchments cannot be compared, because of uncertainty where differences between catchments originate. 


\section{Soil connectivity}

The clayey soils in the studied catchments are susceptible to surface crusting, but due to the abundance of large macro pores from biological activity and cracking, no overland flow occurs until the soil is saturated (Giménez et al., 2012b). This dominance for saturation overland flow was reflected in the soil connectivity component by using the antecedent soil moisture conditions as input for the models. High antecedent wetness indicates low storage capacity, which leads to overland flow earlier and for a longer time for the same precipitation event than it will for low antecedent wetness conditions. Antecedent wetness can be measured with soil moisture sensors, but because no long records of soil moisture data were available for the study areas, a surrogate was needed. The Antecedent Precipitation Index (Kohler and Linsley, 1951) was used as a surrogate in this study

$A P I=\sum_{t=o}^{-i} P_{t} k^{-t}$

where API is Antecedent Precipitation Index ( $\mathrm{mm}), \mathrm{P}$ the precipitation on day $\mathrm{t}(\mathrm{mm}), \mathrm{k}$ a calibration factor, and $t$ is the number of days before $t=0$ that are still included in the index. The model for antecedent precipitation was calibrated using a regression analysis of available soil moisture data from Latxaga on API. For La Tejeria and Oskotz no soil moisture data were available. The best model parameters $\mathrm{k}$ and $\mathrm{t}$ were assessed using an approach where all possible combinations of $\mathrm{k}$ and $\mathrm{t}$ were tested and the combination with the highest $\mathrm{R}^{2}$ was taken and visually inspected for plausibility. The number of days $\mathrm{t}$ incorporated into the model was 17 and the decay factor $\mathrm{k}$ was optimised to 0.98 (Figure 4.2). The values for $\mathrm{t}$ and $\mathrm{k}$ were also used in the calculation of the antecedent wetness in the other catchments, because of lack of soil moisture data for those catchments.

\section{Biological connectivity}

The agricultural catchments Latxaga and La Tejeria comprise agricultural fields, shrubland and some built-up areas. Agricultural fields compose $\sim 90 \%$ of the catchments, of which the large majority is used for cereals. The agricultural activities (sowing, harvesting) and crop growth have a large influence on the biological component of connectivity. The growth of natural vegetation in Oskotz plays an important role in the interception of precipitation and, therefore, on the detachment of sediment. Growth of crops and grasses in the few agricultural fields of Oskotz causes the connectivity in those fields to change similar to the connectivity in Latxaga and La Tejeria.

Vegetation changes throughout the year and strongly influences the reflectance of the surface. The Normalised Difference Vegetation Index (NDVI; Lillesand et al., 2014) was selected in this study as an indicator for crop maturity and vegetation growth, which can be calculated from satellite imagery as follows

$$
N D V I=\frac{N I R-R E D}{N I R+R E D}
$$


where NDVI is the Normalised Difference Vegetation index (-), NIR the top of atmosphere reflectance in the near infrared wavelength bands (-) and RED the top of atmosphere reflectance in the red wavelength band $(-)$.

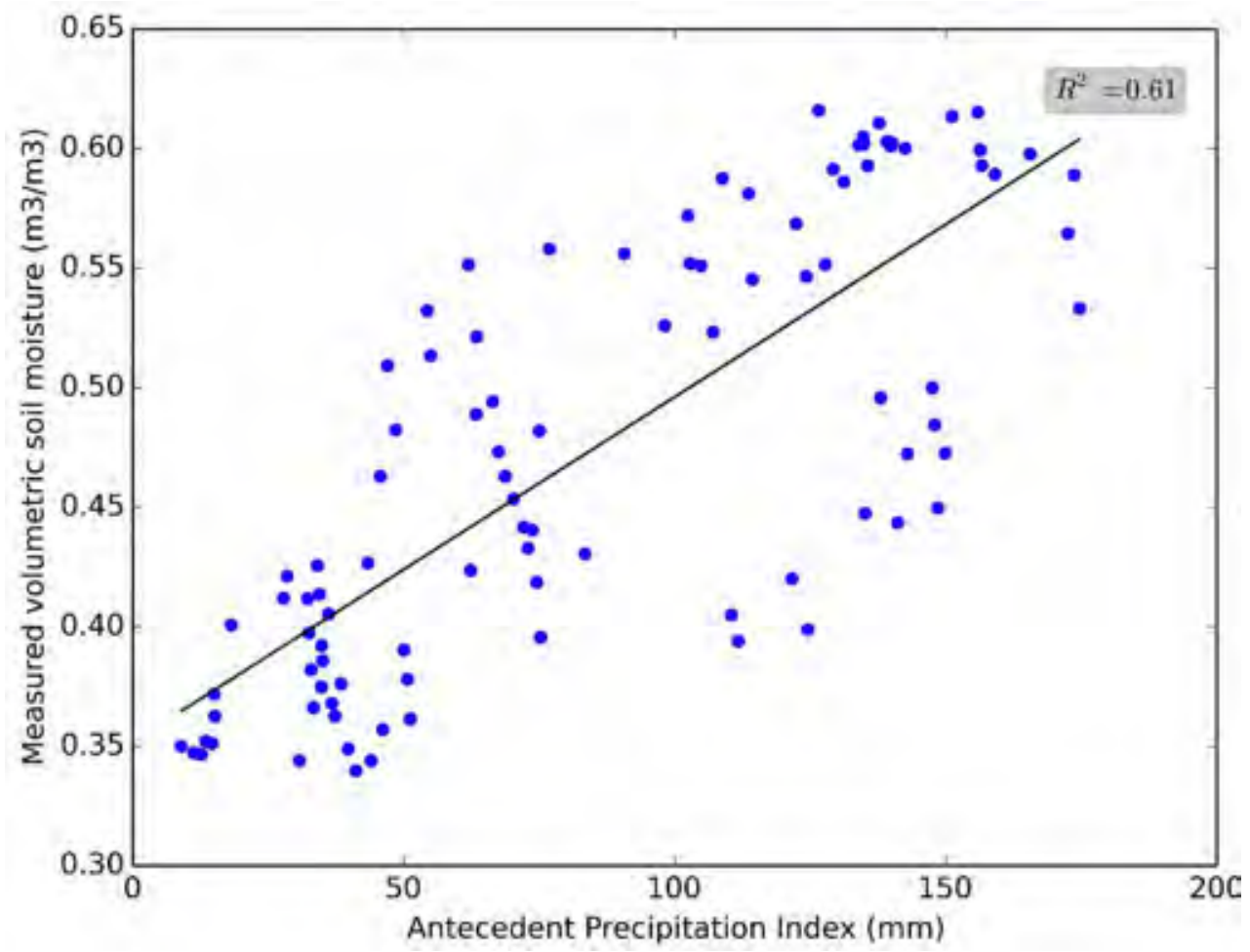

Figure 4.2 Measured soil moisture and calculated Antecedent Precipitation.

A total of 541 scenes of Landsat Thematic Mapper 4-5 (TM4-5) and Enhanced Thematic Mapper plus (ETM+) from 1984 to 2014, with a resolution of 30m were available (Table B1). Scenes from ETM+ were also used after 2003, when the Scan Line Corrector broke down (United States Geological Survey, 2015). This means that only part of the catchments is available for analysis; however, the remaining data for the catchments was considered to be representative for the catchments as a whole, because the missing data were distributed equally over the catchments.

The catchments were clipped from the larger scenes and for each scene the cloud cover within the catchment was determined. To calculate cloud cover, top of atmosphere reflectance for each scene was calculated from the original radiance data using the atmospheric data given with each scene, using the 'Top of Atmosphere Reflectance' tool in SAGA GIS (2.1.3). Cloud cover for all scenes within the catchments was assessed using the Automated Cloud Cover Assessment algorithm in SAGA GIS (Irish, 2000). Scenes where cloud cover within the catchments exceeded $20 \%$ were discarded. Monthly 
average NDVI values were calculated from the remaining scenes. The number of usable scenes (Table 4.2) in La Tejeria is higher than in the other two catchments due to its situation in two overlapping Landsat paths, doubling the amount of available scenes without cloud cover.

Average NDVI values for each catchment (Figure 4.3) show large variations with time in the two agricultural catchments, while the Oskotz forested catchment has a more stable NDVI throughout the year. In Latxaga and La Tejeria, winter wheat is the most abundant crop. Winter wheat starts to grow when it is sown in October and then stays dormant during the winter months, to start growing again around the end of winter. The plants stay green until June, when they ripen and are harvested around the beginning of July. These stages are visible in the NDVI values of the two agricultural catchments. For Oskotz, the leaf growth of the forest starts later in the year than the growth of the winter wheat in Latxaga and La Tejeria, meaning that the NDVI increase starts later, while staying high all throughout the summer.

Table 4.2 Number of scenes with less than $20 \%$ cloud cover per catchment.

\begin{tabular}{lccc}
\hline Catchment & TM5 & TM7 & Total \\
\hline Latxaga & 28 & 62 & 90 \\
La Tejeria & 60 & 143 & 203 \\
Oskotz & 23 & 57 & 80 \\
\hline
\end{tabular}

To use the NDVI values as a measure for the biological connectivity throughout the year, daily values were interpolated from the calculated monthly values. High NDVI values indicate dense vegetation cover and, therefore, low connectivity. The reciprocal of the NDVI values was, therefore, taken as a measure for biological connectivity.
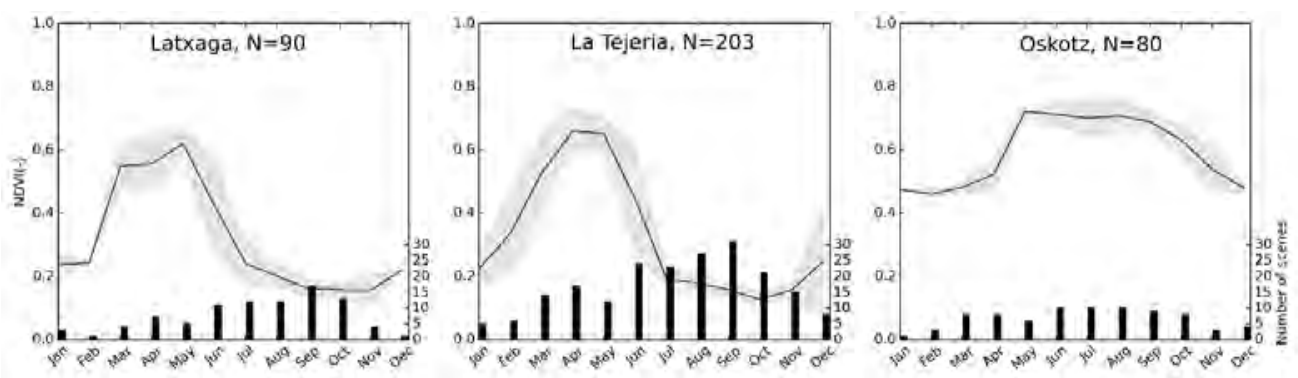

Figure 4.3 Mean NDVI for all three catchments for the period 1984-2014. Number of scenes used per month is indicated by bars. Grey envelope indicates the standard deviation from the mean for each month.

\subsubsection{Combining the components to calculate catchment connectivity}

We assume the highly simplified view that for a given precipitation event $\mathrm{i}$, discharge $\mathrm{Q}$ at the outlet of a given (small) catchment is dependent on the connectivity within the catchment while suspended-sediment yield SSY is dependent on both the amount of sediment detached and the connectivity within the catchment. Sediment detachment in 
turn is dependent on soil characteristics, soil cover and precipitation. We assumed that for a single catchment the soil characteristics remained constant during the study period and that soil cover is already represented in the biological component of connectivity. We state, therefore, that the suspended-sediment yield at the outlet for a given event $\mathrm{i}$, or day d, depends on the precipitation and on the connectivity within the catchment on that day. The exact relationship between the connectivity components, precipitation and discharge and sediment yield is unknown and differs between catchments and might differ between events. To be able to assess the possibilities for our datasets, however, we opted for a linear system, where all components were normalised and, therefore, weigh equally in the determination of catchment connectivity.

$\mathrm{C}_{\mathrm{S}}$ and $\mathrm{C}_{\mathrm{B}}$ were normalised in time using a unity based normalisation. This was done to be able to better assess the importance of the different components. The components were normalised as follows

$X^{\prime}=\frac{X_{i}-X_{\min }}{X_{\max }-X_{\min }}$

Where $\mathrm{X}^{\prime}$ is the data point i normalized between 0 and $1, X_{i}$ each data point I, $X_{\text {Min }}$ the minimum value among all the data points within a single catchment and $\mathrm{X}_{\mathrm{Max}}$ the maximum value among all the data points within a single catchment. After the unitybased normalisation all model parameters were log-transformed before using linear regression because of non-normal distributions in the data. After regression, the results were back transformed.

A hydrological model and a sediment-delivery model were created on daily timescale using the calculated connectivity components. Both models were created by applying a multiple ordinary least squares regression on datasets of the separate catchments. The linear regression model is as follows

$S S Y / Q=\beta_{1} C_{S}+\beta_{2} C_{B}+\beta_{3} P+c$

where SSY/Q is the suspended-sediment yield $\left(\mathrm{kg} \mathrm{ha}^{-1}\right.$ day $\left.^{-1}\right)$ or discharge $\left(\mathrm{mm} \mathrm{day}^{-1}\right)$, $\beta$ are the regression coefficients that determine the slope of the three individual model parameters: connectivity components $\mathrm{C}_{\mathrm{s}}$ and $\mathrm{C}_{\mathrm{B}}$, and the precipitation $\mathrm{P} ; \mathrm{c}$ is a constant.

Two random years were omitted from the model calibration and used for validation; only days with precipitation ( $\mathrm{P}>0$ ) were used. Model efficiency for both the calibration and validation periods were assessed using the Nash-Sutcliffe efficiency coefficient (Nash and Sutcliffe, 1970) a method for estimating model efficiency commonly used in hydrology, but applicable to sediment-yield models as well (Arnold et al., 1995). The coefficient is calculated as follows:

$E_{f}=1-\frac{\sum_{t=1}^{T}\left(Y_{O}^{t}-Y_{M}^{t}\right)^{2}}{\sum_{t=1}^{T}\left(Y_{O}^{t}-\bar{Y}_{O}\right)^{2}}$ 
where $E_{f}$ is the Nash-Sutcliffe model efficiency (-), $Y_{O}^{t}$ the observed discharge/SSY at time step $\mathrm{t}, Y_{M}^{t}$ the modelled discharge/SSY at time step $\mathrm{t}$ and $\overline{Y_{O}}$ the mean measured discharge/SSY.

The value $E_{f}=1$ indicates perfectly modelled values, $E_{f}=0$ indicates the model prediction values are as good as the mean of the observed values and $E_{f}<0$ occurs when the mean of the observed values is a better predictor than the model.

Separate model components $\left(\mathrm{C}_{\mathrm{S}}, \mathrm{C}_{\mathrm{B}}\right.$ and $\mathrm{P}$ ) were incrementally added to the model to assess the sensitivity of adding/subtracting a single factor. In total 7 combinations for each catchment and for both the discharge models and the sediment-yield models were tested, resulting in a total of 42 different models.

Besides the validation of the two random years that were excluded, model parameters were estimated and the models were then validated by using a leave-one-out crossvalidation method, by consecutively taking one year out of the whole dataset and validating the models for the year that was taken out. This was done for all years $(\mathrm{n}=$ 13) for all catchments. For both the parameter estimation period and the validation year, the Nash-Sutcliffe model efficiencies were calculated.

\subsection{Results}

Measured relative discharge patterns throughout the year for all three catchments are similar due to equal meteorological forcing in all three catchments (Figure 4.4). Figure 4.4 shows measured discharges and specific sediment yield (SSY) as monthly averages per day for visualisation purposes. Suspended-sediment yield per surface area in La Tejeria is, however, up to $1400 \%$ greater than the sediment yield in Latxaga and Oskotz. The highest sediment yields for all catchments occur in late winter/early spring.
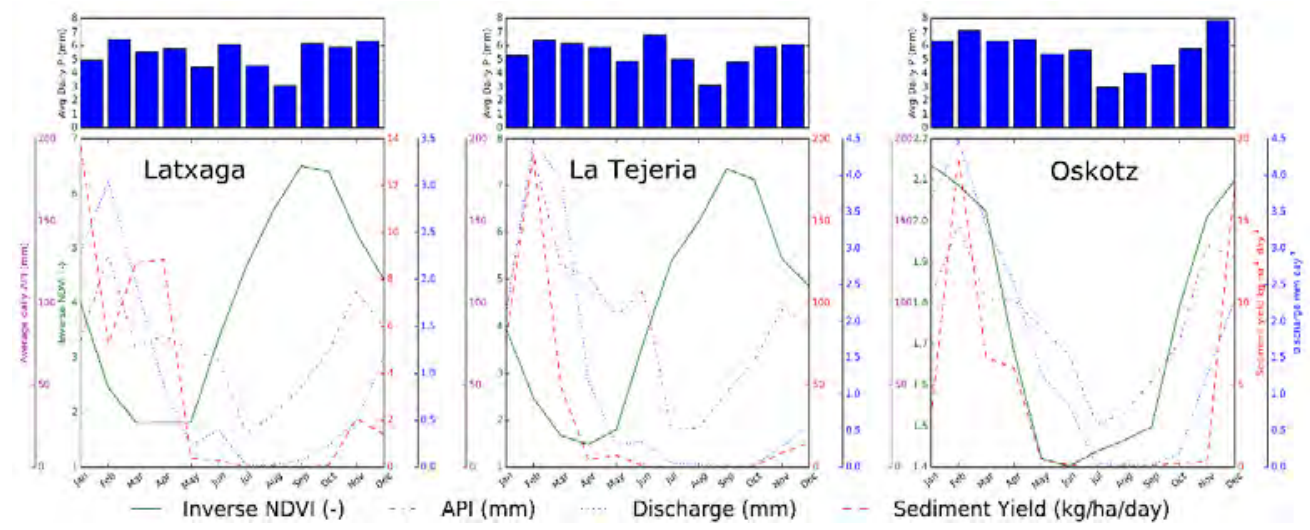

Figure 4.4 Average daily values per month, of precipitation, inverse NDVI, antecedent precipitation index (API), discharge and sediment yield for the years where the model parameters were estimated.

The inverse NDVI (biological component) for Latxaga and La Tejeria has little resemblance to either discharge or sediment yield, indicating that it is not a dominant factor controlling the discharge or sediment output (Figure 4.4). The inverse NDVI for 
Oskotz, however, shows a higher resemblance, suggesting a relation between the biological connectivity component and discharge and sediment yield. The antecedent precipitation index (soil component) and the average daily precipitation both show reasonable resemblance to both discharge and sediment yield for all catchments.

The model results for the individual model components (Table 4.3) show that for the discharge model for the Latxaga $(E=0.28)$ and La Tejeria $(E=0.3)$, agricultural catchments the soil connectivity component has the highest model efficiency, while for the Oskotz forest catchment this is the case for precipitation ( $\mathrm{E}=0.32)$. The sedimentyield model results show a different image; the sediment yield is more closely related to the precipitation than to the soil connectivity component for Latxaga $(\mathrm{E}=0.2)$ and La Tejeria (E=0.14). For Oskotz the differences between the two are small. The combinations of the components and precipitation show that model efficiency increases for every component that gets added; for all cases, the highest model efficiency occurs when all components are included (Table 4.3). Adding the biological component to the model $\left(\mathrm{PC}_{\mathrm{s}} \mathrm{vs} . \mathrm{PC}_{\mathrm{B}} \mathrm{C}_{\mathrm{s}}\right)$, has little influence on model efficiency for the discharge models, and even less for the sediment-yield models.

The discharge models with all components included show high similarity with the measured values (Figure 4.5). For months with high measured discharge, modelled values are lower than the measured values. For months with low measured discharge, modelled values are higher than the measured values. For sediment yield, the differences between measured and modelled values change per catchment; for Latxaga the modelled sediment yield peak in February is higher than measured values, for La Tejeria the modelled sediment peak is lower than measured values and for Oskotz the modelled sediment yield peak is similar to measured values (Figure 4.5). Model equations show that in Latxaga and La Tejeria there is a negative correlation between the biological connectivity and the discharge, which can also be visually observed in the graphs of the individual connectivity components (Figure 4.4).

Table 4.3: Nash-Sutcliffe efficiency coefficients for the various linear models for discharge and sediment yield.

\begin{tabular}{lcccccc}
\hline \multirow{2}{*}{ Components } & \multicolumn{3}{c}{ Discharge } & \multicolumn{3}{c}{ Sediment Yield } \\
\cline { 2 - 7 } & Latxaga & La Tejeria & Oskotz & Latxaga & La Tejeria & Oskotz \\
\hline P & 0.16 & 0.12 & 0.32 & 0.2 & 0.14 & 0.08 \\
CB & -0.12 & -0.11 & 0.06 & -0.02 & -0.02 & 0.01 \\
CS & 0.28 & 0.3 & 0.27 & 0.06 & 0.06 & 0.06 \\
CBCS & 0.34 & 0.35 & 0.29 & 0.07 & 0.06 & 0.12 \\
PCB & 0.22 & 0.22 & 0.39 & 0.23 & 0.17 & 0.11 \\
PCS & 0.39 & 0.54 & 0.51 & 0.28 & 0.29 & 0.21 \\
PCBCS & 0.5 & 0.62 & 0.55 & 0.3 & 0.29 & 0.23 \\
\hline
\end{tabular}



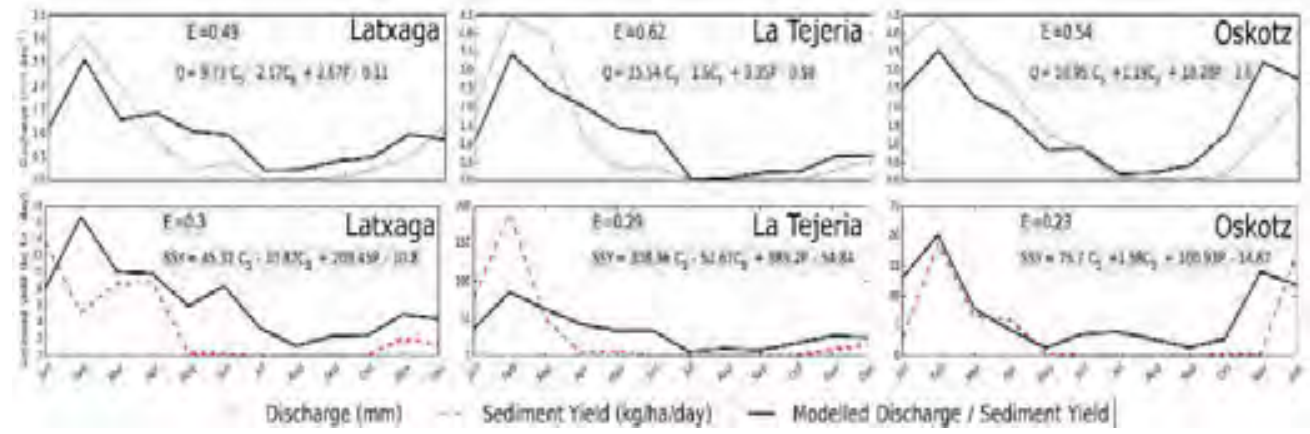

Figure 4.5 Average daily values for measured discharge and modelled discharge (top) and measured sediment yield and modelled sediment yield (bottom). Associated Nash-Sutcliffe coefficients (E) are given per catchment, per model.

The modelled daily discharge values have higher similarity to the measured values when there has been little antecedent precipitation (Figure 4.6, top left). The similarity decreases when there is a sequence of events and when precipitation amounts are high (Figure 4.6, top right). Similarly, for sediment yield, better results are obtained after a period with relatively little rain, whereas a consecutive number of events cause for larger differences in modelled and measured sediment yield (Figure 4.6, bottom).

Results of model validation (Figure 4.7) show a similar pattern to model calibration, where the discharge models have higher Nash-Sutcliffe efficiency coefficients than the sediment-yield models. All Nash-Sutcliffe efficiency coefficients for the discharge model are positive $(0.4$ to 0.47$)$ while for the sediment-yield models the coefficients are all negative $(-5.77$ to -0.54$)$. For the discharge models there is an underestimation of discharge, which is especially noticeable for Latxaga. For the sediment-yield models there is for all catchments an overestimation of sediment yield.

Results for the leave-one-out cross-validation show that, on average, the models for La Tejeria have the highest Nash-Sutcliffe model efficiency for predicting discharge, while the models for Oskotz have the highest efficiency for suspended sediment prediction (Table 4.4). Results show, furthermore, that the discharge models are less sensitive than the SSY models which is illustrated by the lower differences between the minimum and maximum values and a smaller standard deviation. The high standard deviation for the validation of discharge of La Tejeria is caused by a single year with an extremely low Nash-Sutcliffe coefficient (2012: -997.15) which is caused by many days with measured discharges close to zero, while precipitation amounts were high $(\mathrm{P}>10 \mathrm{~mm})$, causing an overestimation of modelled discharge. 

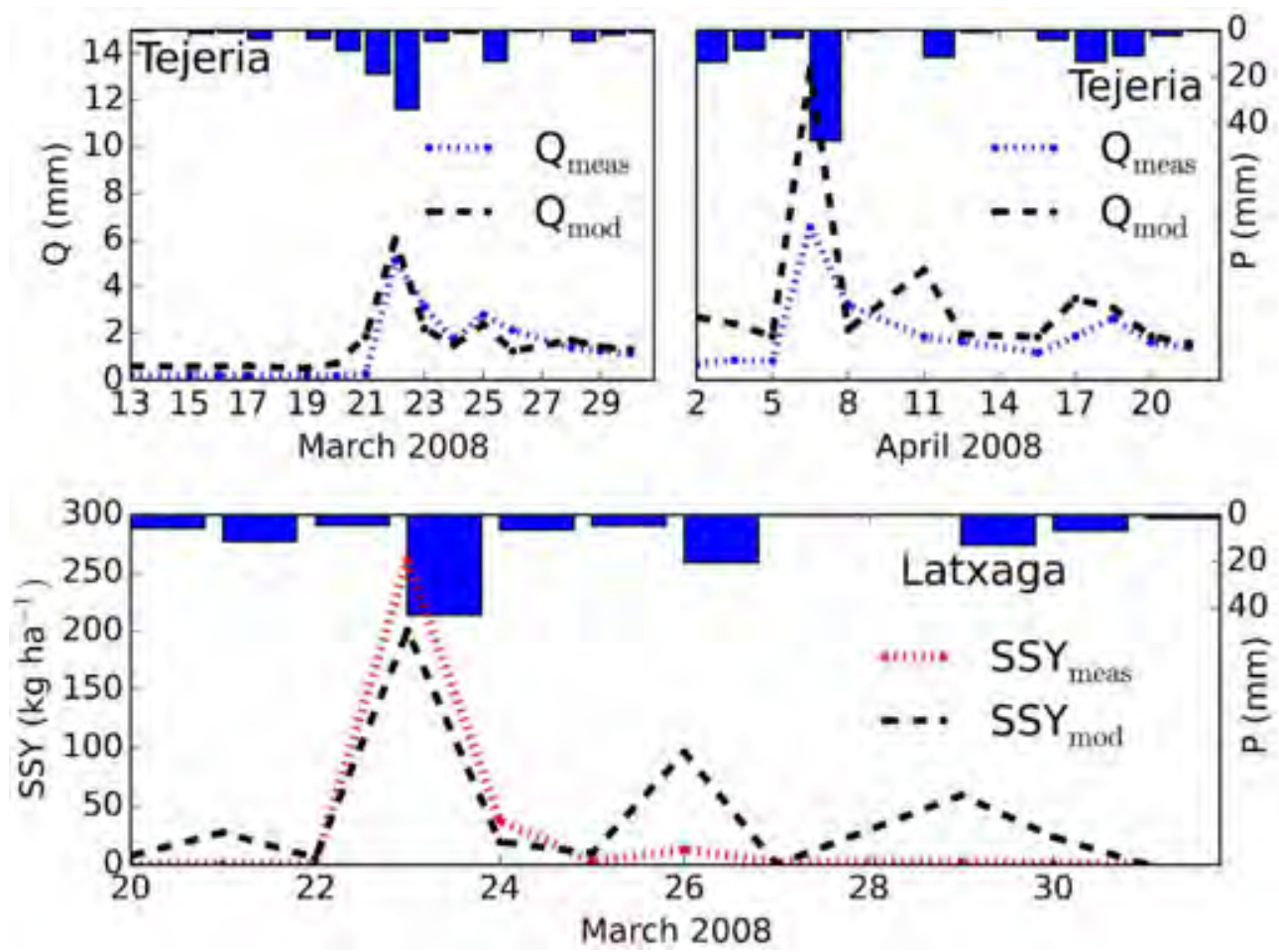

Figure 4.6 Examples of daily measured $\left(\mathrm{Q}_{\text {meas }}\right)$ and modelled $\left(\mathrm{Q}_{\mathrm{mod}}\right)$ dscharge La Tejeria (top) and measured (SSY ${ }_{\text {meas }}$ ) and modelled (SSY ${ }_{\text {mod }}$ ) sediment yield for Latxaga (bottom). 


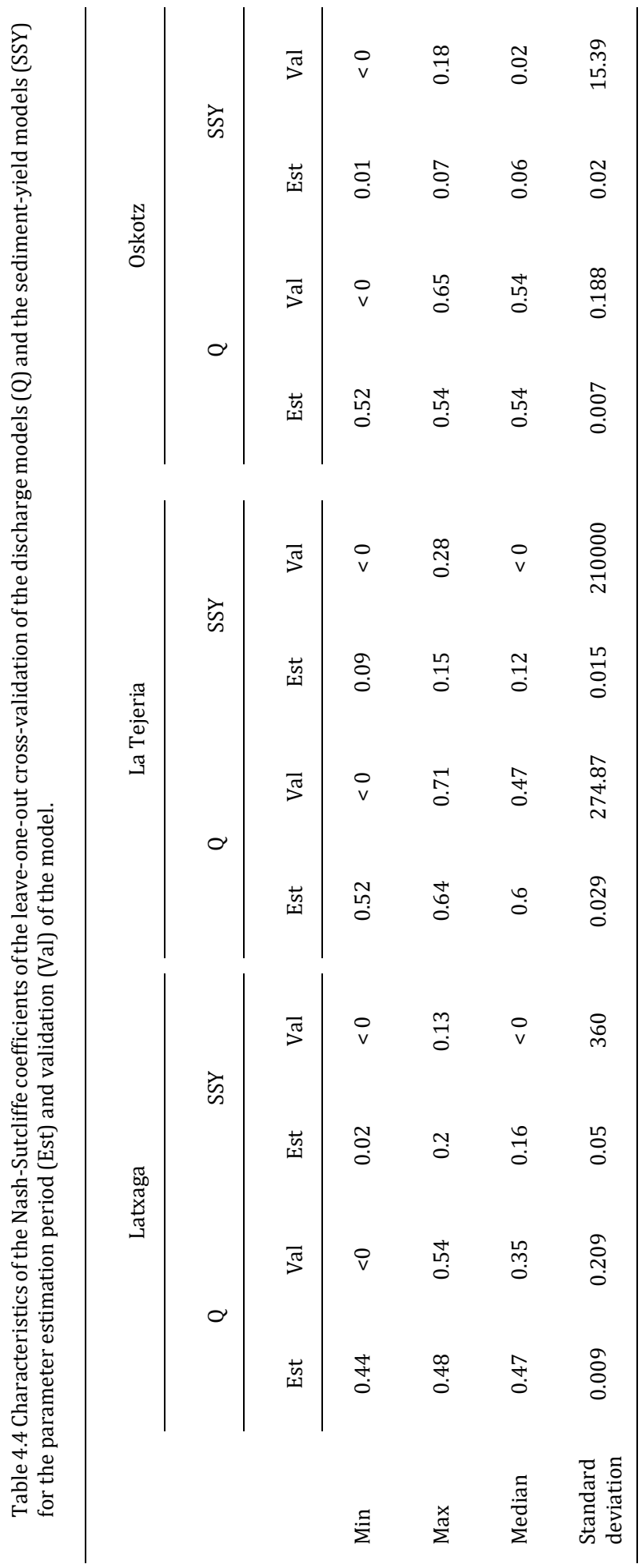



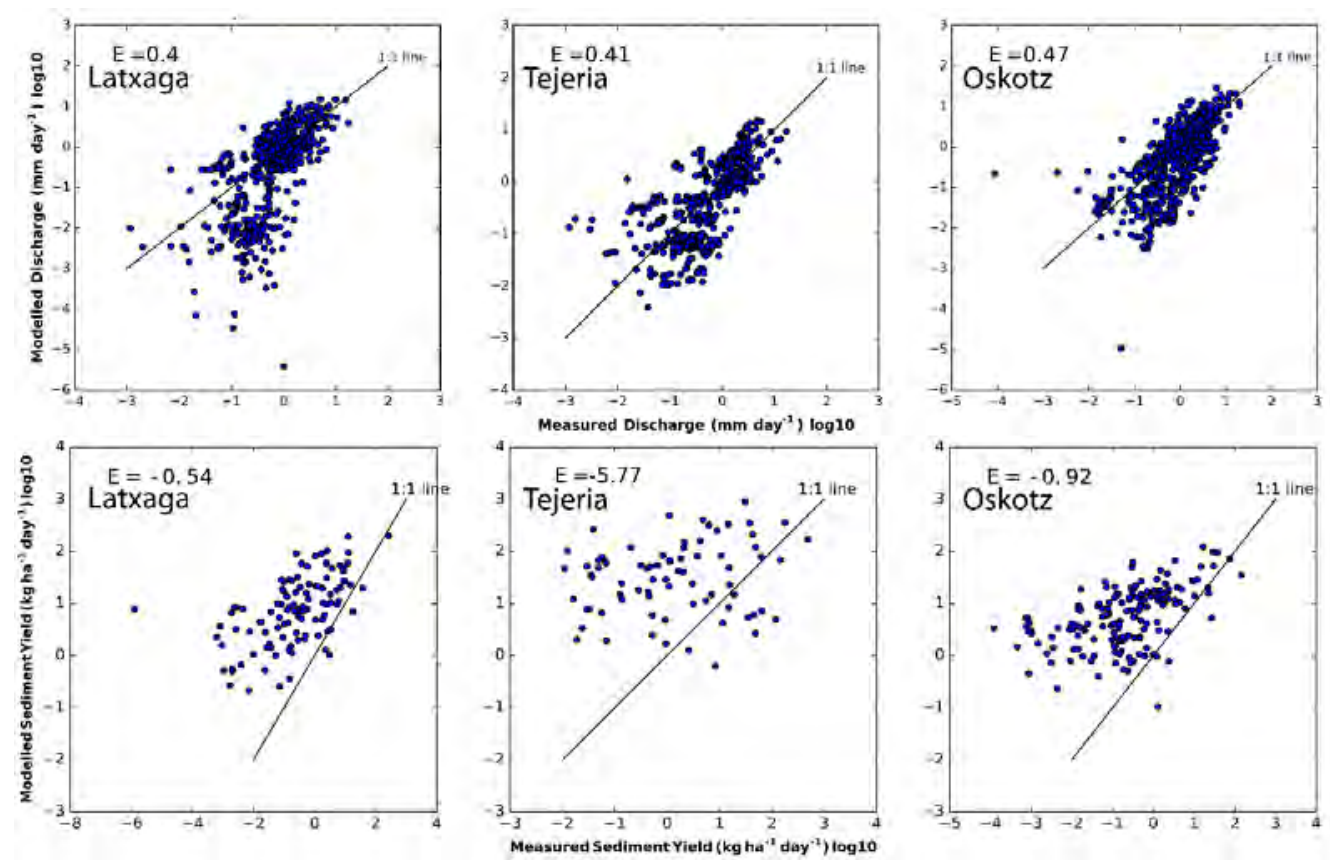

Figure 4.7 Model validation results for discharge (top) and sediment yield (bottom), using two years which were omitted from model calibration.

\subsection{Discussion}

The results of the linear models showed that the discharge models perform well, for both the model calibration period (Figure 4.5) as well as the validation period (Figure 4.7). This shows that with relatively little information about the catchment and little to no information about the spatial arrangement of geomorphological features a good estimate of discharge on a daily basis can be made. Predicted discharge values were higher than measured values for low flow periods, while they were underestimated for periods with high discharge. This difference between predicted values and measured values is most likely due to the usage of a linear model, which cannot deal well with high or low extremes, because these have less weight in the regression when compared to (many) values near the average. For the sediment-yield models the results were less satisfactory, especially for the validation of the model. In our specific case the model performs worse than the mean of the data $(\mathrm{E}<0)$. Erosion/sediment-yield models more often have difficulties, leading to an underestimation of large events or overestimation of small events (Nearing, 1998), which is also the case with our model. Chahor et al. (2014) experienced similar difficulties predicting sediment yield on a daily basis for the Latxaga catchment with a distributed, physically-based model. Improvement might be possible with a non-linear model that expresses the non-linear nature of the response of erosion to overland flow, such as used in more mechanistic landscape models (Lesschen et al., 2009; Coulthard et al., 2012). 
The daily model results show that the model is sensitive to precipitation input and antecedent wetness (Figure 4.6). High precipitation amounts result for both the discharge models and the SSY models in an overestimation of discharge and SSY. An exception is the SSY on March 23, 2008 (Figure 4.6, bottom), which shows a higher measured SSY than modelled SSY. This might be caused by a 'washing' out of sediments from the stream bed as also suggested by Bracken et al. (2015). Small events deliver sediments to the stream, but with those events there is not enough stream power to deliver the sediments to the outlet of the catchment. A large event then washes out all the sediment from the bed and the system 'resets'.

In this study, we used discharge and SSY as proxies for connectivity, while these might not fully represent the connectivity in the area. Because the catchments are relatively small and homogeneous, we assumed that the whole of the catchments contributed equally to the sediment delivered to the outlet (Parsons et al., 2006). However, even in small catchments sediment yield at the outlet is not only dependent on the detachment of sediment, but also on the deposition, and the spatial distribution of the sediment deposition. It would therefore be beneficial to our understanding of sediment connectivity to have a combination of data gathered at relatively small scales (plot/hillslope) at multiple locations and at large scales (catchment).

The connectivity components were assessed separately for predicting discharge and suspended-sediment yield, which showed that precipitation is the most important factor influencing the sediment yield, followed by the soil connectivity component. Similarly, Giménez et al. (2012b) showed this in a principal component analysis of factors influencing sediment output in Latxaga. They found that runoff and discharge are the most important factors, followed by antecedent conditions, where the antecedent discharge had a higher loading in the principal component analysis than the antecedent precipitation. In a connectivity perspective these results show that changes in vegetation throughout the year have little influence on the connectivity of the entire catchment, although it may contribute in complex ways. The results show that antecedent soil moisture plays a large role for connectivity, which previous studies also concluded (Western et al., 2001; Meyles et al., 2003; Seeger et al., 2004; Butzen et al., 2011).

The multiple linear regression method for discharge performed best for La Tejeria (E = $0.62)$, followed by Oskotz ( $E=0.54$ ) and Latxaga ( $E=0.49$; Figure 4.5). For the validation period, however, the Oskotz model performed better than the Latxaga model and the La Tejeria model (Figure 4.7). This is also shown in the leave-one-out cross-validation method, where Oskotz has a higher mean for the validations. Oskotz also has a lower standard deviation for both the discharge model and the SSY model, which shows that the inter-annual changes in connectivity in Oskotz are smaller than in Latxaga and La Tejeria and is also an indication that during the course of a year the connectivity in Oskotz changes less than in Latxaga and La Tejeria.

Similar problems with predicting sediment yields at the basin scale, due to not including all processes involved in sediment erosion and transport were shown in other studies 
(de Vente and Poesen, 2005; de Vente et al., 2006). The same data were used to show that the sediment yield in La Tejeria is higher than in Latxaga, although climate, catchment size and soils are similar for both catchments (Giménez et al., 2012a). Differences in suspended-sediment yield might be caused by e.g. the shape of the catchment and bank failures/erosion but also unrecorded human activity such as tillage and construction of (sub-) surface drains and roads. These human influences especially play a large role in the two agricultural catchments, perhaps differently so in Latxaga than in La Tejeria. Additionally, there is barely any riparian vegetation or channel vegetation in La Tejeria. This lack of vegetation promotes bank failure and erosion, creating more sources of sediment than in Latxaga and Oskotz, as previously mentioned by Casalí et al. (2008).

The antecedent precipitation index (API; Kohler \& Linsley, 1951) as a surrogate for measured soil moisture is a relatively easy and straightforward method to assess antecedent moisture conditions. There are, however, also some limitations of the method. The decay factor $\mathrm{k}$ is catchment and season specific and it would, therefore, be preferable to have a different $\mathrm{k}$ value for every catchment for every season. No soil moisture data were available for all seasons for each catchment, therefore, it was impossible to calibrate the $\mathrm{k}$ and $\mathrm{t}$ values. This lack of data might have led to errors in the estimation of the antecedent moisture, especially for Oskotz and La Tejeria. For future work other methods for estimating antecedent moisture may be used (Anctil et al., 2004; Brocca et al., 2008). Antecedent soil moisture can be modelled spatially using statistical models (Western et al., 2001) or by using novel methods with indices like the topographic wetness index (Beven and Kirkby, 1979; Yong et al., 2012).

The biological component of connectivity was negatively correlated to discharge and sediment yield for Latxaga and La Tejeria. This is due to land use of the catchments; Latxaga and La Tejeria are almost completely covered with cereal crops, while Oskotz is mainly forest. The growing stages of the cereals (see 2.3.3) are reflected in the Normalised Difference Vegetation Index (NDVI). The usage of NDVI to determine the biological connectivity has high potential, because so much satellite imagery is available and the process is easily automated. Results may improve, however, when the spatial distribution of the NDVI or vegetation in general is also taken into account (Bergkamp et al., 1996; Bracken and Croke, 2007). One method to add the spatial distribution is to combine NDVI values per grid cell with their distance to the nearest river. High density vegetation close to a river might have larger influence on connectivity than vegetation far away from a river (Correll, 2005). Using the NDVI in this way opens up the possibility of separating the growth of e.g. riparian vegetation and agricultural crops and assessing the influence these types of vegetation have on connectivity separately. Another option to include the influence of vegetation on connectivity is to include it in a way similar to (Vigiak et al., 2012), who used an index of connectivity to determine hillslope sediment delivery ratios, where vegetation is represented as the Revised Universal Soil Loss Equation (RUSLE) C-factor. Instead of using the RUSLE C factor, actual biomass data or e.g. remote sensing data could be used to serve as input for an index of connectivity (Foerster et al., 2014). 
The topographical component was not assessed in this study, but for future work, data from unmanned aerial vehicles or laser altimetry may provide a useful source of data. These data in combination with techniques like object based image analysis might provide an effective way to describe the topography, patterns and presence of landforms and so describing the topographical connectivity component. Much exploratory work on geomorphological mapping using OBIA has already been done (e.g. Anders et al., 2013). These high resolution geomorphological maps can then be used as input to determine homogeneous areas and the connections between these areas. These homogeneous areas could then function as nodes within a network, with connections (edges) to adjacent areas. The edges can represent e.g. buffering areas like natural shrub vegetation in agricultural plots, riparian vegetation or floodplains. This way many data are necessary to estimate model parameters for all areas as well as for calibration of the edge (buffering) parameters. Likewise, we assume that there is no residence time for sediments, because we a look at connectivity on a daily basis. This might not pose a large problem for the catchments in this study because of their size, but for larger catchments this might be resolved by subdividing the catchment into a network of nodes and edges.

This study assessed the value of existing data to understand connectivity changes at the catchment scale. It can conceivably be improved by adding spatially explicit data and assessing the connectivity within the catchments. The ultimate goal of understanding sediment pathways, sinks and residence times could benefit greatly from new methods for measuring landscape parameters and sediment transport distances.

\subsection{Conclusion}

The topographical, biological and soil components were evaluated to assess the connectivity of water and sediments. The components were used as input in a linear model for discharge and suspended sediment for three catchments using existing data. The results of the application of the components show that good prediction results could be obtained for discharge at the outlet, but less so for sediment yield. Future work using this approach needs to incorporate the spatial arrangement of the different features within the catchment and include non-linear responses to achieve better prediction results for sediment transport. 


\section{Acknowledgements}

This project was funded by the Dutch Organisation for Scientific Research (NWO) grant number 822.01.004 and by the Spanish Ministry of Science and Innovation, project number CGL2011-24336. We would like to thank Jokin del Valle de Lersundi of the Department of Rural Development and Environment of the Government of Navarre (Spain) for help in obtaining the sediment data. 


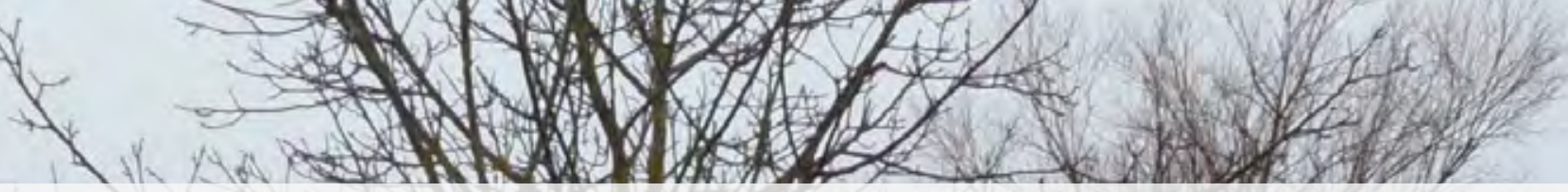

\section{Chapter 5}

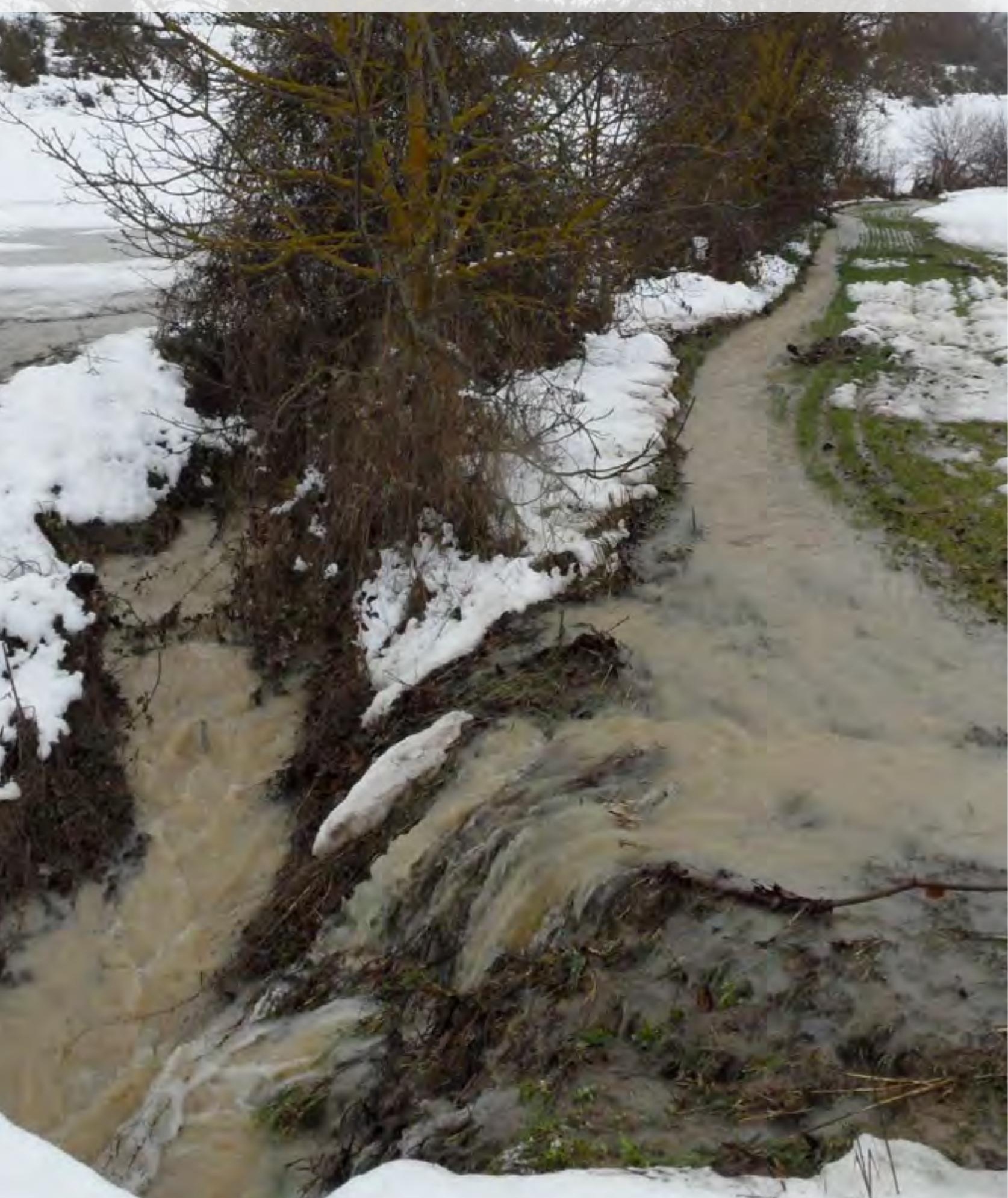




\begin{abstract}
To mitigate negative effects associated with soil erosion when implementing management strategies, it is important to understand catchment sediment dynamics. One way of improving our understanding of catchment sediment dynamics is by using models. Models are often only calibrated and validated at the outlet, with which water and sediment sources remain unconstrained and may be wrongly modelled. To correctly model sediment dynamics there is a need for models that can incorporate measured data at multiple spatial scales. The objective of this study, therefore, was to better understand the implications of model calibration at different spatial scales on the simulation of hydrology and sediment dynamics of an agricultural catchment. We applied the LAPSUS-D model to the agricultural catchment of Latxaga $\left(2.07 \mathrm{~km}^{2}\right)$ in NSpain. The model was calibrated and validated (4 years: 2011-2015) using three datasets at varying spatial scales: hillslope, catchment and the combined dataset (combined-calibrated model). The hillslope-calibrated model showed mainly infiltration-excess overland flow, the catchment-calibrated mainly saturation-excess overland flow at the footslopes and the combined-calibrated model showed saturationexcess overland flow from the midslopes to the footslopes. For hydrology, the combined-calibrated model simulated the large discharge peaks best, while at the hillslope scale, the hillslope-calibrated model performed best. Hillslope-calibrated model had the highest model efficiencies for sediments, for calibration (0.618) and validation (0.269). Hillslope-calibrated model was the only model that showed observed gully erosion on a high-resolution DEM and displayed channel sediment dynamics. However, absolute quantities of erosion and deposition within the catchment were too high. The results show that modellers need to be aware of problems associated with automatic calibration, over-calibration and not incorporating measured data at multiple spatial scales. We advocate incorporating runoff and sediment tracing data at multiple scales whenever this is possible and to carry out specific measuring campaigns towards this end.
\end{abstract}

Submitted as: Masselink RJH, Temme AJAM, Keesstra SD, Casalí, J, Campo-Bescós, MA, Nunes, JP. Scale matters: contrasting hillslope and catchment scale calibration results for hydrology and sediment fluxes using the LAPSUS-D model. Earth Surface Processes and Landforms. 


\section{Chapter 5. Scale matters: contrasting hillslope and catchment scale calibration results for hydrology and sediment fluxes using the LAPSUS-D model}

\subsection{Introduction}

Soil erosion is associated with many negative onsite and offsite effects, e.g. the loss of fertile topsoil, siltation of waterways and reservoirs and eutrophication of surface waters (Morgan, 2009). To mitigate these negative effects when implementing management strategies, it is important to understand the catchment sediment dynamics (Walling and Collins, 2008; Mekonnen et al., 2015a). One way to better understand these dynamics is through the connectivity concept.

Connectivity describes how water and associated matter such as sediments and nutrients moves from a source through the catchment, to a (semi-) permanent sink (Wainwright et al., 2011; Bracken et al., 2015; Poeppl et al., 2016). Connectivity helps to better understand how transport processes interact at various spatial and temporal scales. In return, this improved understanding of the system can lead to better management practices by e.g. catchment managers and farmers (García-Orenes et al., 2009; Jones et al., 2014; Keesstra et al., 2016).

One way of getting a better understanding of the functioning of a catchment and predicting water and sediment discharge is by using models. Models have been applied to simulate water and sediment transport at the plot scale (Nord and Esteves, 2005), hillslope scale (Brazier et al., 2001), and catchment scale (de Roo et al., 1996; Coulthard et al., 2012; Temme et al., 2013; Keesstra et al., 2014b).

On the one hand, connectivity has been explicitly applied as an input variable to models: as a connectivity function for describing sub-grid surface roughness (Antoine et al., 2009; Peñuela et al., 2012), by using a connectivity index as model input (Cavalli et al., 2013) or using connectivity components to describe various factors influencing connectivity as model variables (Masselink et al., 2016). On the other hand, connectivity on hillslopes and catchments has been an output of models using changing (artificial) topography (Baartman et al., 2013) or differences in vegetation (Coulthard and Van De Wiel, 2016). These models are normally calibrated and validated using data obtained at one support level: catchment, hillslope or plot level (Merritt et al., 2003).

When only using data at the outlet for model calibration and validation, the prediction of the sources of water and sediment remains unconstrained and may be wrong. Many different combinations model parameters could lead to similar results at the outlet, which is known as equifinality (Brazier et al., 2000). In reality, water discharge during events could be dominated by overland flow or by fast throughflow of water already stored in soil before the event (Sayama and McDonnell, 2009). Sediments, similarly, can originate from the channel bed or banks close to the outlet, or from the hillslopes further away. Sediment sources can even change during events, which has been demonstrated using hysteretic loops (Seeger et al., 2004; Giménez et al., 2012b; Sherriff 
et al., 2016). Moreover, it has been shown that models calibrated at the outlet often underestimate large soil losses and overestimate small soil losses (Nearing, 1998).

To determine where sources of water and sediment are, and how they travel through the catchment, there is a need for models that can incorporate measured data at multiple spatial scales or from different sources (Nunes et al., 2009; Finger et al., 2015). If these models can reproduce the measured data over this range of scales, more confidence can be placed in their simulation of actual water and sediment source areas, transfer paths and sinks throughout a whole catchment. Consequently, better management decisions can then be taken at the appropriate locations.

The objective of this study, therefore, was to better understand the implications of model calibration at different spatial scales on the simulation of hydrological and sediment connectivity of an agricultural catchment. Sub-objectives were to:

- Calibrate and validate a model for runoff at multiple scales and calibrate these models for sediment yield;

- Assess which effects these multi-scale calibrations have on predicting runoff and sediment connectivity by comparing modelled runoff and sediment yield to measured quantities and observed spatial patterns of erosion and deposition.

\subsection{Methods}

\subsubsection{LAPSUS-D model}

For this modelling study, we chose to use the LAPSUS-D model. The model allows for spatially-explicit modelling of soil erosion processes, while having low data needs. LAPSUS (LAndscape ProcesS modelling at mUlti-dimensions and scaleS) is a reduced complexity model, originally developed for modelling landscape evolution due to soil erosion at long ( $>100$ years) timescales (Schoorl et al., 2000). Later, the model was adapted to model landscape evolution due to e.g. tillage (Baartman et al., 2012c), creep, solifluction (Temme and Veldkamp, 2009) and landslides (Claessens et al., 2009). More recently, LAPSUS was rewritten to work on a daily timescale, (Keesstra et al., 2014b). It is this model version, LAPSUS-D (Figure 5.1), which we use here in adapted form. A detailed description of the LAPSUS and LAPSUS-D model can be found in Baartman et al. (2012b), Keesstra et al. (2014) and Schoorl et al. (2000). Below we describe the adaptations we have made to the model.

In previous versions of LAPSUS and LAPSUS-D, spatially uniform evaporation was used, which was simply subtracted from the precipitation and/or the storage for each day. For better modelling of soil moisture evolution and soil moisture variations we included spatially distributed evaporation with the double crop coefficient method (Allen et al., 1998), which calculates the actual crop adjusted potential evaporation based on the calculated reference evapotranspiration using the Penman-Monteith method (Allen et al., 1998). The crop coefficients, crop cover coefficients and soil moisture availability then determine the actual evaporated amount of water. 
To realistically model flow in the (un)saturated zone, the flow in the soil layer was calculated on the basis of soil moisture above field capacity. Whenever soil moisture reaches below field capacity, ground water flow is set to 0 and storage is only further reduced by evaporation.
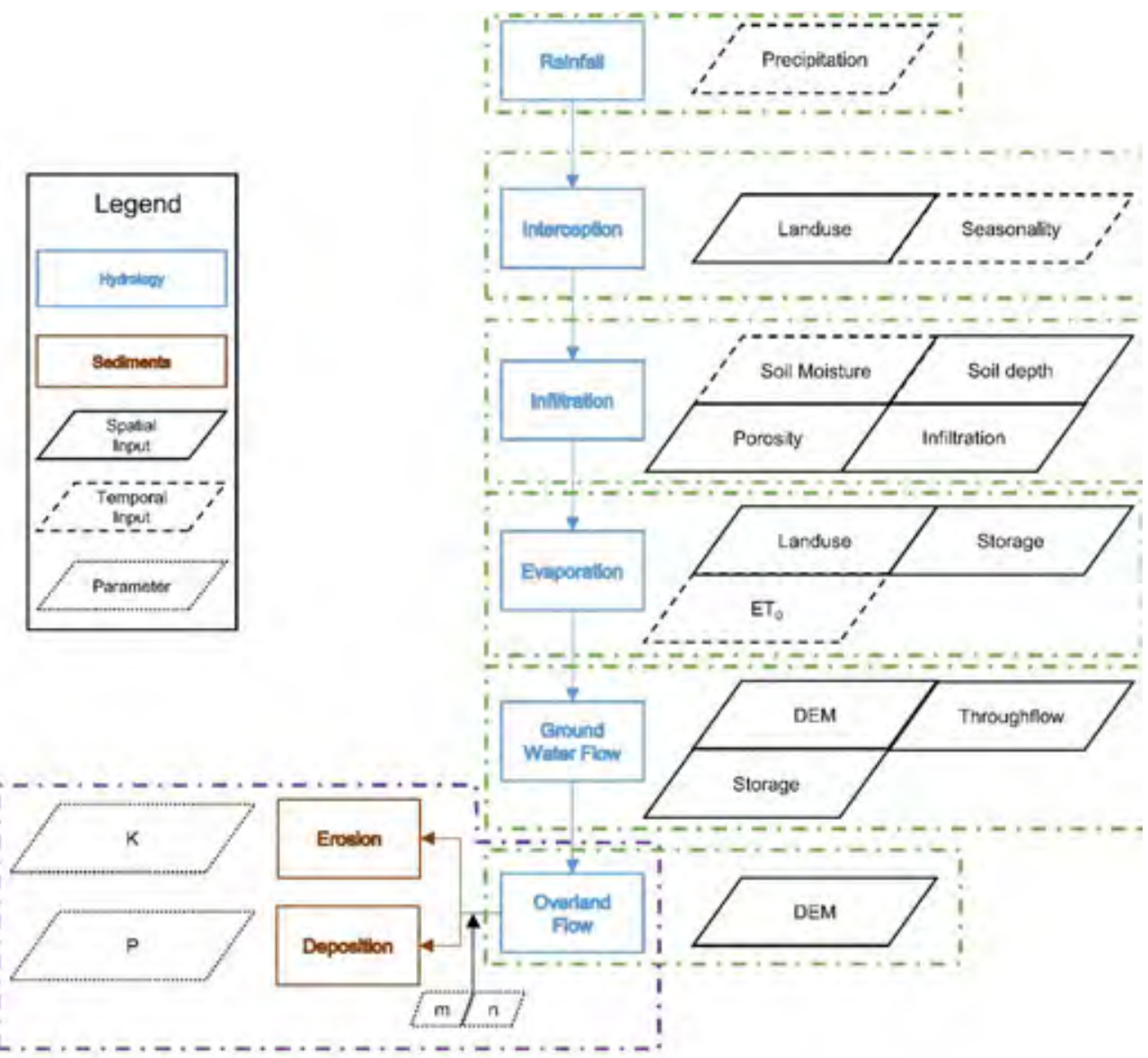

Figure 5.1 Flow chart of the steps of the LAPSUS-D model. The individual steps in the flowchart are described in e.g. (Schoorl et al., 2000; Keesstra et al., 2014b). Parameters K, P, $m$ and $\mathrm{n}$ in the erosion section of the figure can also be varied spatially for e.g. different land use types. Similarly, spatial parameters like infiltration, throughflow, soil depth, land use, storage and porosity can be made spatially uniform where appropriate. 


\subsubsection{Study area and available data}

The study area of Latxaga (207 ha) is located in Northern Spain in the autonomous region of Navarre (Figure 5.2). Bedrock in the catchment is predominantly marl (Gobierno de Navarra, 1997) which after weathering results in soils with high clay and silt content. Climate is humid Sub Mediterranean with average annual precipitation of $835 \mathrm{~mm}$ (Gobierno de Navarra, 2001). Most of the precipitation falls in winter, as low intensity rainfall and snowfall. In summer, convective storms can occur with high precipitation intensities.

The catchment is predominantly used for agriculture $(\sim 90 \%)$ and the remainder consists of semi-natural shrub vegetation and some roads and built-up area. The majority of the fields are sown with winter wheat (Triticum spp.) and barley (Hordeum vulgare L.), whereas some farmers use crop rotation and may plant e.g. sunflower (Helianthus) in spring. The streams in the study area are covered by large amounts of riparian vegetation (grasses, shrubs and trees) (Figure 5.2b).

a

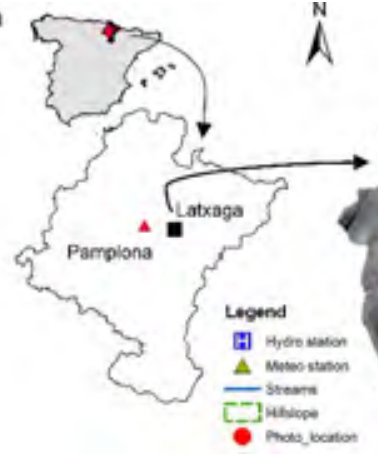

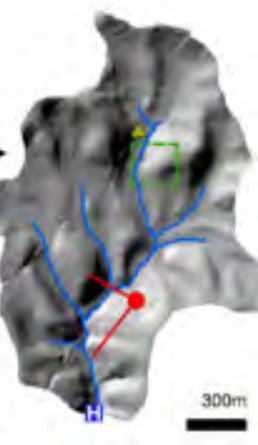

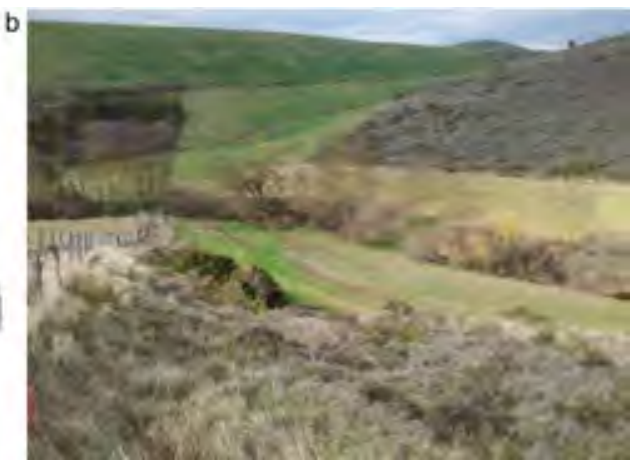

Figure 5.2 a) The Latxaga study area) (N-Spain), showing the subcatchment and the hillslope where overland flow measurements were taken. Red lines next to photo location indicate the viewing direction of the photo.

b) Photo of the Latxaga catchment, showing the mixture of agricultural fields, semi-natural shrub areas and riparian vegetation with trees along the stream.

\section{Data}

For topography, a digital elevation model with a resolution of 5 meters, obtained by means of aerial laser altimetry data (Gobierno De Navarra, 2014) was available for input into the LAPSUS-D model (Figure 5.3). Furthermore, three digital elevation models obtained with an unmanned aerial vehicle with resolutions of $10 \mathrm{~cm}$ were available for the assessment of modelled erosion and deposition patterns (Masselink et al., 2017). Land use (Figure 5.3) was extracted from the land use maps of the government of Navarre (Gobierno de Navarra, 2001). Meteorological data have been collected in $10 \mathrm{~min}$ intervals in the catchment since 2002 (Chahor et al., 2014). A hydrological station is located at the outlet of the catchment, where level measurements are taken every 10 minutes using a UNIDATA model 6531 sampler (UNIDATA, Willetton, Australia).

Overland flow data was available for a hillslope within the Latxaga catchment (Figure 5.2a) for the winters of 2012-2013 and 2014-2015 (for details see Masselink et al., 
2016a). Overland flow detection sensors were placed on the hillslope and the connectivity of the hillslope (fraction between $0-1$ ) was calculated for every 10 minutes using networks. Overland flow quantities (in $\mathrm{mm}$ ) were determined by multiplying the mean daily overland flow connectivity fraction by daily precipitation. The hillslope represents about 0.85 ha or $\sim 0.41 \%$ of the entire catchment.
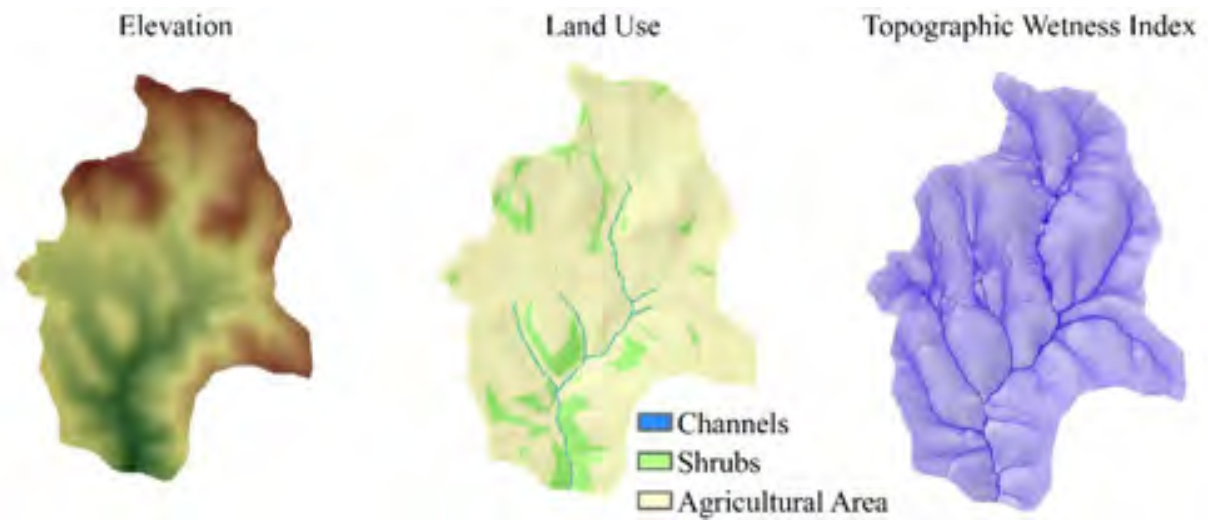

Figure 5.3 Catchment characteristics of the Latxaga catchment, showing the elevation, Land use and the calculated Topographic Wetness Index.

At the hydrological station, 4 water samples per day were taken using an automatic sampler (ISCO 6712, Teledyne Isco, Lincoln, NE, USA). The samples were mixed to obtain daily average sediment concentrations. Average daily sediment concentrations were multiplied by daily total discharge to obtain total sediment discharge.

Because the data availability at the hillslope scale is limited to the period 2012-2015, those four years were used as a dataset for the modelling experiments (Table 5.1). Annual precipitation sums range from 709 to $1696 \mathrm{~mm}$ (Table 5.1).

Table 5.1 Maximum daily, weekly, monthly and yearly cumulative precipitation (in $\mathrm{mm}$ ) for four hydrological years. 30 year mean annual precipitation is $876 \mathrm{~mm}$. Hydrological data availability (shaded cells) for the various hydrological years at the different spatial scales are also given.

\begin{tabular}{lcccccc}
\hline \multirow{2}{*}{ Hydrological Year } & \multirow{2}{*}{ Daily } & Weekly & Monthly & Yearly & \multicolumn{2}{c}{ Data availability } \\
\cline { 6 - 7 } & & & & & Hillslope & Catchment \\
\hline $2011-2012$ & 43.3 & 88.5 & 192.2 & 709.0 & \\
$2012-2013$ & 93.1 & 215.0 & 371.5 & 1695.7 & \\
$2013-2014$ & 40.6 & 118.1 & 167.4 & 1161.6 & \\
$2014-2015$ & 54.8 & 115.4 & 233.1 & 895.3 & \\
\hline
\end{tabular}

\subsubsection{Model parametrization}

Interception values were set for two parts of the year: summer (ordinal day numbers 109-272) and winter. Interception values for winter wheat were taken from Kang et al. (2005), who found that maximum interception is around $1 \mathrm{~mm}$, while in the beginning 
of the growing season it is around $0.2 \mathrm{~mm}$. Interception for the semi-natural shrub areas was set to $3 \mathrm{~mm}$ for summer and $1.5 \mathrm{~mm}$ in winter. Both values fall within the range of Mediterranean shrubs (Garcia-Estringana et al., 2010) and were chosen to reflect the mixture of grasses, shrubs and trees in these areas.

Crop transpiration and bare soil evaporation were calculated using the double cropcoefficient method developed by the United Nations Food and Agriculture Organisation (FAO) (Allen et al., 1998). Reference evapotranspiration was calculated using the Penman-Monteith equation by using the data of the meteorological station in the catchment. The station did not include an air pressure sensor. Air pressure data was used from Pamplona airport at $\sim 12 \mathrm{~km}$ distance from the catchment. The difference in air pressure due to altitude difference ( $125 \mathrm{~m}$ lower for the airport) was corrected using the ideal gas law:

$P_{h}=P_{0} e^{(-m g h) /(R T)}$

where $\mathrm{P}_{\mathrm{h}}$ is the pressure at point $\mathrm{h}(\mathrm{kPa}), \mathrm{P}_{0}$ the pressure at the reference point, $\mathrm{m}$ the molecular mass of air $\left(0.0289644 \mathrm{~kg} \mathrm{~mol}^{-1}\right)$, g the gravitational constant, $\mathrm{h}$ the difference in altitude $(\mathrm{m}), \mathrm{R}$ the gas constant $\left(8.314462 \mathrm{~J} \mathrm{~K}^{-1} \mathrm{~mol}^{-1}\right)$ and $\mathrm{T}$ the absolute temperature (in K).

The parameters necessary for the calculation of the evaporation are i) wilting point and field capacity, ii) readily and total available water for evaporation, iii) rooting depth and iv) crop properties and cover. Wilting point was set at a volumetric water content of $0.357 \mathrm{~m}^{3} / \mathrm{m}^{3}$, which is the average reported by Chahor et al. (2014) for the catchment. Readily available water was set at $10 \mathrm{~mm}$ and total available water at $25 \mathrm{~mm}$, which corresponds to average values for silty clay loam (Allen et al., 1998). Rooting depth for winter wheat was set at $1.4 \mathrm{~m}$ (Allen et al., 1998), or to the soil depth where this was less than $1.4 \mathrm{~m}$. The vegetation characteristics were taken from provided FAO table (Allen et al., 1998; Figure C1).

\section{Sediment transport parameters}

Sediment transport in the LAPSUS-D model is controlled by 4 parameters: erodibility K, sedimentability $\mathrm{P}$ and streamflow parameters $\mathrm{m}$ and $\mathrm{n}$. Only $\mathrm{K}, \mathrm{P}$ and $\mathrm{m}$ were used for calibration and $\mathrm{n}$ was set to 2 , which is characteristic for overland flow and concentrated flow (Kirkby, 1971).

\section{Model calibration and validation}

The entire dataset (2011-2015) was split into a calibration period and a validation period. The hydrological years 2011-2012 and 2012-2013 were used for calibration, and 2013-2014 and 2014-2015 were used for validation. Precipitation and evaporation data for one previous year (2010-2011) were used as spin-up time for the model. Calibration and validation were performed sequentially for hydrology and sediment. This was possible because the amount of sediment transported does not affect the hydrology. 
During calibration, hydrological model outputs were compared to observations at two scales: hillslope and catchment. The parameters that were calibrated against hydrological data were soil depth, infiltration rate and throughflow rate. These parameters were chosen for calibration because no catchment wide data on these was available for the parametrisation of the model.

Soil depths in the catchments are shallower for the slopes than for the valley bottoms (Giménez et al., 2012b). To reflect this variation, we predicted soil depth using the Topographic Wetness Index (TWI)(Beven and Kirkby, 1979). TWI was calculated for the entire catchment and was multiplied by a calibration parameter. The range of TWI for the Latxaga catchment is between 3 and 15 (not including channels; Figure 5.3). Values for the calibration parameter were chosen between 0.05 and 0.20 to obtain a range of realistically possible soil depths (between 15 and $75 \mathrm{~cm}$ for factor 0.05 and 60 and $300 \mathrm{~cm}$ for calibration factor 0.20 ). A total of 4 soil depth calibration factors were used in calibration: $0.05,0.1,0.15$ and 0.2 . (Table 5.2)

For each soil depth calibration factor a Monte Carlo type calibration was used; for both the infiltration and the throughflow parameter 200 values were randomly sampled and model performance using these parameter values was then evaluated. The values were sampled between 0-1 m day-1 for infiltration. In a previous study it was recognised that the throughflow parameter can be up to an order of magnitude higher than the infiltration parameter (Keesstra et al., 2014b). We verified that the throughflow parameter above $4 \mathrm{~m}$ day $^{-1}$ did not change the model efficiency values, and the range for value sampling for throughflow was, therefore, set at 0-4 m day-1. The model then ran with these random parameter settings and the model efficiency of each of the parameter combinations was assessed at hillslope scale, at catchment scale and for the combined dataset.

Model efficiency was calculated using Nash-Sutcliffe efficiency (Nash and Sutcliffe, 1970):

$E_{f}=1-\frac{\sum_{t=1}^{T}\left(Y_{O}^{t}-Y_{M}^{t}\right)^{2}}{\sum_{t=1}^{T}\left(Y_{O}^{t}-\overline{Y_{O}}\right)^{2}}$

where $E_{f}$ is the Nash-Sutcliffe model efficiency (-), $Y_{O}^{t}$ the observed discharge at time step $t, Y_{M}^{t}$ the modelled discharge at time step $\mathrm{t}$ and $\overline{Y_{O}}$ the mean measured discharge.

The parameter combinations were plotted and interpolated to obtain a full parameter space for the selected calibration ranges. These parameter spaces show the sensitivity of the infiltration and throughflow parameters throughout their ranges. The highest scoring parameter combinations for the hillslope scale, catchment scale and the combined dataset were subsequently used for the model calibration for sediments.

The model calibration for sediments was done using measured values of sediment export at the catchment outlet. The parameters that were calibrated in the model are the exponent $\mathrm{m}$, the erodibility factor $\mathrm{K}$ and the sedimentability factor $\mathrm{P}$ (Table 5.2). These calibration factors were chosen because the modelled sediment discharge is most 
sensitive to these parameters, and the effect of changing these parameters has not been previously evaluated for the LAPSUS-D model (Baartman et al., 2013).

Table 5.2 Range of calibration parameters used for calibration of hydrological and sediment part of the LAPSUS-D model.

\begin{tabular}{lcc}
\hline & Hydrology & Sediments \\
\hline Soil depth factor $(\overline{S D} \mathrm{a})$ & $0.05-0.2(0.38-1.52 \mathrm{~m})$ & \\
Infiltration & $0-1 \mathrm{~m}$ & \\
Throughflow & $0-4 \mathrm{~m}$ & \\
& & \\
$\mathrm{~m}$ & & $1-2$ \\
Kagr & $0-1$ \\
Pagr & $0-1$ \\
Kshrub & $0-$ Kagr \\
Pshrub & Pagr-1 \\
\hline
\end{tabular}

a Mean catchment soil depth

The m parameter was sampled between 1 and 2 with increments of 0.1 . The agricultural areas and the semi-natural shrub areas have different properties in respect to erodibility and sedimentability. Due to higher vegetation cover in shrub areas, sediments will not be detached easily and due to higher roughness overland flow velocities drop and sediments settle. To reflect this in the model, during the calibration procedure first $K$ and $P$ for the agricultural areas $\left(K_{a g r}\right.$ and $\left.P_{a g r}\right)$ were randomly sampled, after which $K$ and $P$ for the shrubs area $\left(K_{\text {shrub }}\right.$ and $\left.P_{\text {shrub }}\right)$ were randomly sampled. To ensure the differences in erodibility the $\mathrm{K}$ factor for shrubs was randomly sampled between 0 and the $\mathrm{K}$ factor for agriculture, while the $\mathrm{P}$ factor for shrubs was randomly sampled between the $P$ factor of agriculture and 1 . For every m parameter 400 random parameter combinations of $\mathrm{K}_{\mathrm{agr}}, \mathrm{K}_{\text {shrub}}, \mathrm{P}_{\mathrm{agr}}$ and $\mathrm{P}_{\text {shrub }}$ were sampled.

To determine the effect of calibration of a sediment transport model at the catchment scale, the model was calibrated three times for sediments: with optimum hillslope discharge parameter settings, with optimum catchment discharge parameter settings and with optimum combined (hillslope + catchment) parameter settings. Model efficiency for sediments was also determined using the Nash-Sutcliffe model efficiency.

\section{Determining differences in sediment connectivity due to calibration scales}

The hillslope-calibrated, catchment-calibrated and combined-calibrated models were used to compare the measured and modelled values of sediment discharge. We compared the models for their sediment output at the outlet of the catchment and compared the spatial patterns of erosion and deposition to measured and observed erosion and deposition patterns from the high-resolution digital elevation models. Furthermore, we compared the amount of sediments in the pixels defined as channels to assess the sediment dynamics within the channel for each model. 


\subsection{Results}

\section{Hydrology}

Results for model calibration for hydrology show that the optimum Nash-Sutcliffe model efficiencies (MEF) are obtained with different parameter combinations for the hillslope and the catchment scale (Figure 5.4). At shallow soil depths, the catchmentcalibrated model does not produce results with MEF higher than 0, while for the hillslope scale nearly all combinations have an MEF of more than 0.3 . The highest MEF can be found for both the hillslope scale and the catchment scale at a mean catchment soil depth of $1.14 \mathrm{~m}$ (soil depth calibration factor $=0.15$ ). For all soil depths, the catchment scale MEFs are mirrored to the hillslope scale MEFs. This mirrored effect is especially noticeable in the mean soil depths of $1.14 \mathrm{~m}$ and $1.52 \mathrm{~m}$. Apparently, good simulation results at the catchment scale to some extent prevent good results at the hillslope scale and vice versa.
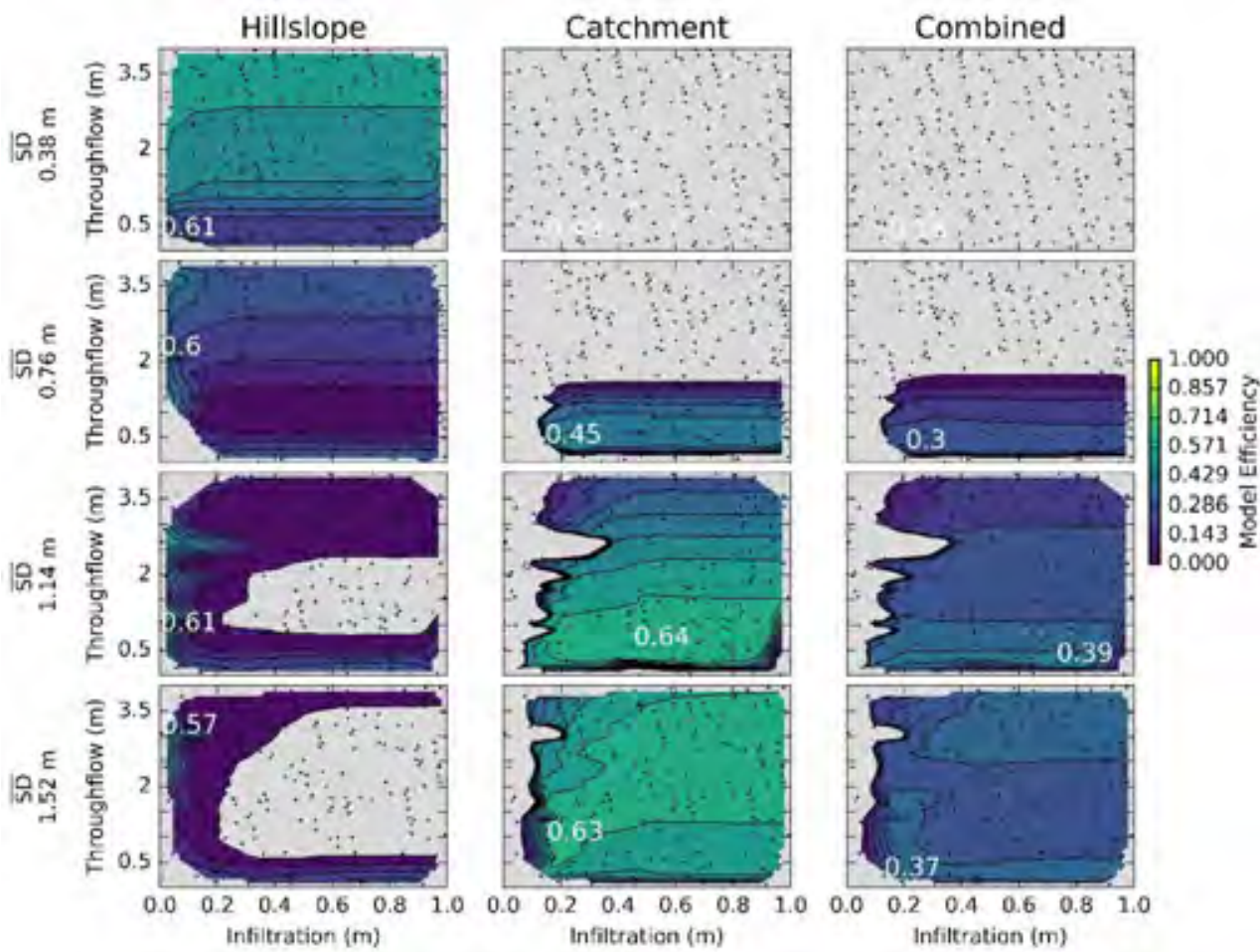

Figure 5.4 Nash-Sutcliffe model efficiency for different calibration parameter sets for the Latxaga catchment at two spatial scales for the calibration period 2012-2013. Rows in the figure depict different calibration factors for soil depth, with $\overline{S D}$ depicting mean catchment soil depth (m). Dots indicate modelled parameter combinations; colours represent interpolated values between modelled values. White numbers are highest model efficiencies, located at the respective parameter combination. Non-coloured areas equal model efficiencies lower than 0 .

The best infiltration parameter is $0.001 \mathrm{~m}$ for the hillslope scale, while it is $0.45 \mathrm{~m}$ for the catchment scale (Table 5.3). Throughflow parameters are not substantially different, although the throughflow parameter at the hillslope scale has little influence 
on the actual discharge; very little water infiltrates and is therefore not available for subsurface flow. Model efficiency remains largely equal when the throughflow parameter is increased while the infiltration parameter is low (Figure 5.4). The mean MEF for the calibration period is similar for both the hillslope scale and the catchment scale. The mean MEF for the validation period at the hillslope scale is larger than for the calibration period $(0.701$ vs 0.614$)$, while at the catchment scale it is smaller than for the calibration period ( 0.404 vs 0.639$)$. When the different model calibrations are validated at other scale levels (Table 5.4) the combined-calibrated model performs best at the catchment scale $(\mathrm{MEF}=0.464)$.

Table 5.3 Optimum parameter settings for water discharge for calibration period (2011-2013) and individual model efficiencies for the parameter settings for each individual hydrological year and for the two-year period for the model calibration and validation.

\begin{tabular}{lccccccccc}
\hline & \multicolumn{3}{c}{ Calibrated parameter value } & \multicolumn{3}{c}{ Calibration } & \multicolumn{3}{c}{ Validation } \\
\hline Calibrated & & Infiltration & Throughfl & '11- & '12- & '11- & '13- & '14- & '13- \\
for & $\overline{S D}(\mathrm{~m})$ & $(\mathrm{m})$ & ow $(\mathrm{m})$ & $' 12$ & $' 13$ & $' 13$ & $' 14$ & $' 15$ & $' 15$ \\
\cline { 2 - 11 } Hillslope & 1.14 & 0.001 & 0.891 & & 0.614 & 0.614 & & 0.701 & 0.701 \\
Catchment & 1.14 & 0.454 & 0.604 & 0.169 & 0.607 & 0.639 & 0.321 & 0.507 & 0.404 \\
Combined & 1.14 & 0.648 & 0.231 & & & 0.380 & & & 0.250 \\
\hline
\end{tabular}

The hillslope-calibrated model shows a good agreement with the measured hillslope discharge, especially for normal events (Figure 5.5). For large events $(>20 \mathrm{~mm})$, the peaks are underestimated. This underestimation of peaks is also visible for the hydrographs at the catchment scale of the catchment-calibrated model, where similarly, large events are more underestimated than small events. The hydrographs, furthermore, show that the hillslope-calibrated model highly overestimates catchment discharge and the catchment-calibrated model highly underestimates hillslope discharge. The combined-calibrated model performs best at the catchment scale, even for the large event peaks, but it overestimates the small peaks of catchment discharge more than the catchment-calibrated model. The better performance of the combinedcalibrated model was also evidenced by the higher MEF for the combined-calibrated model validated at the catchment scale (Table 5.4).

Table 5.4 Nash Sutcliffe Efficiencies for the LAPSUS-D model calibrated using three different datasets, validated for three different spatial datasets.

\begin{tabular}{llll}
\hline & Hillslope & Catchment & Combined \\
Calibration & & & \\
\hline Hillslope & 0.701 & -7.33 & -3.69 \\
Catchment & 0.04 & 0.404 & 0.263 \\
Combined & 0.08 & 0.464 & 0.250 \\
\hline
\end{tabular}


The hydrological behaviour of the three calibrated models is distinct because of the different parameter combinations. The hillslope-calibrated model has very low infiltration rates, causing mainly infiltration excess overland flow (Hortonian Overland Flow; HOF). This HOF also has no space to infiltrate further downslope, because the soil is so impermeable throughout the catchment. The catchment-calibrated model has higher infiltration rates and lower throughflow rates than the hillslope-calibrated model. The additional infiltration in the catchment-calibrated model causes less infiltration excess runoff, but will cause more throughflow in the soil, resulting in saturated soils downslope and, as a consequence, more exfiltration, leading to Saturation Excess Overland Flow (SOF). The combined-calibrated model has the highest infiltration rates, which saturates the soil quickly over the entire range of the hillslopes. The throughflow parameter of the combined-calibrated model is the lowest of all three models, causing less lateral water movement than in the catchment-calibrated model. This decrease in lateral movement causes the soils to stay saturated longer, causing SOF flow higher up the hillslope than for the catchment-calibrated model.
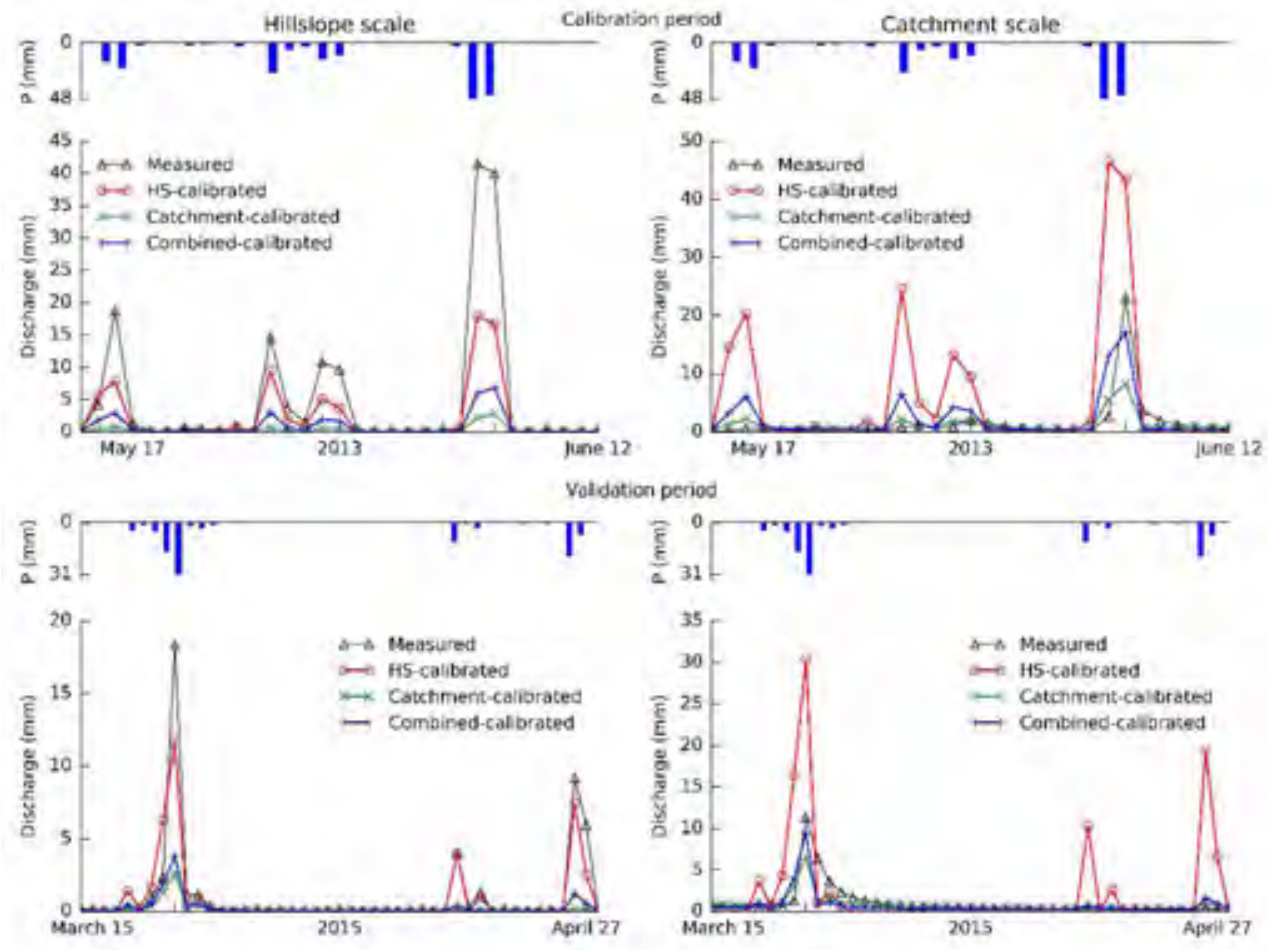

Figure 5.5 Examples of hydrographs of measured discharge, HS (Hillslope)-calibrated, Catchment-calibrated and combined-calibrated model calibrations for the calibration period (top row) and validation period (bottom row) for the hillslope scale (left column) and the catchment scale (right column). 


\section{Sediments}

Both the hillslope-calibrated model and the catchment-calibrated model produce satisfactory results when looking at the model efficiencies for the calibration period (MEF > 0.5; Moriasi et al., 2007)) (Table 5.5). However, the MEF for sediments for the hillslope-calibrated model for 2011-2012 is notably low (MEF < 0), This is most likely due to fewer datapoints, with very low sediment discharge during that year. Because there are only few points, this large negative MEF is not reflected in the MEF for the entire calibration period. The combined-calibrated model is not satisfactory for the calibration period $(\mathrm{MEF}=0.374)$. For the validation period none of the models produces

Table 5.5 Optimum parameter settings for sediment yield and individual model efficiencies for the parameter settings for each individual year and the mean calibration and validation efficiencies.

\begin{tabular}{|c|c|c|c|c|c|c|c|c|c|c|c|}
\hline & \multicolumn{5}{|c|}{ Parameters } & \multicolumn{3}{|c|}{ Calibration } & \multicolumn{3}{|c|}{ Validation } \\
\hline & $\mathrm{m}$ & $\mathrm{K}_{\text {agr }}$ & $\mathrm{K}_{\text {shrub }}$ & $\mathrm{P}_{\text {agr }}$ & $\mathrm{P}_{\text {shrub }}$ & '11-'12 & '12-'13 & '11-'13 & '13-'14 & '14-'15 & '13-'15 \\
\hline Hillslope & 1.5 & 0.300 & 0.136 & 0.521 & 0.786 & -54.089 & 0.660 & 0.618 & 0.131 & 0.549 & 0.269 \\
\hline Catchment & 1.6 & 0.684 & 0.460 & 0.379 & 0.409 & -0.213 & 0.492 & 0.502 & -0.004 & 0.074 & 0.022 \\
\hline Combined & 1.6 & 0.690 & 0.437 & 0.246 & 0.494 & -0.224 & 0.441 & 0.374 & -0.018 & 0.037 & 0.000 \\
\hline
\end{tabular}

satisfactory results. Especially the catchment-calibrated model and the combinedcalibrated model do not perform well with MEFs close to zero. This shows the lack of robustness in the hydrological models, where similarly only the hillslope-calibrated model was the only model that did not decrease its model efficiency for the validation period.

The sedigraphs (Figure 5.6), similarly show that the hillslope-calibrated model produces the best agreement with the peaks of sediment discharge at the outlet. On average, they still underestimate the actual sediment discharge peaks, but for some periods with low measured sediment discharge, for example in 2013 (Figure 5.6), the sediment discharge is overestimated. The sediment dynamics in the channel (Figure 5.6) of the catchment-calibrated model and combined-calibrated model are very small. The sediment in the channel increases initially in 2013 and erodes only slowly (e.g. around February 24, 2015). The channel sediment dynamics of the hillslope-calibrated model are more variable, showing both sediment accumulation as well as erosion in the channel, which are nevertheless small when compared with the initial increase.

The simulated maps of erosion and deposition (Figure 5.7) show that the amounts of erosion and deposition in the hillslope-calibrated model are higher than the erosion and deposition in the catchment-calibrated model and combined-calibrated model. Erosion in the hillslope-calibrated model occurs largely midslope, with the sediment mainly being deposited again on the valley floors and in the channel. For the catchment and combined-calibrated models, the erosion occurs lower on the slope than for the hillslope-calibrated model, whereas deposition occurs in the valley bottoms and the channels. 

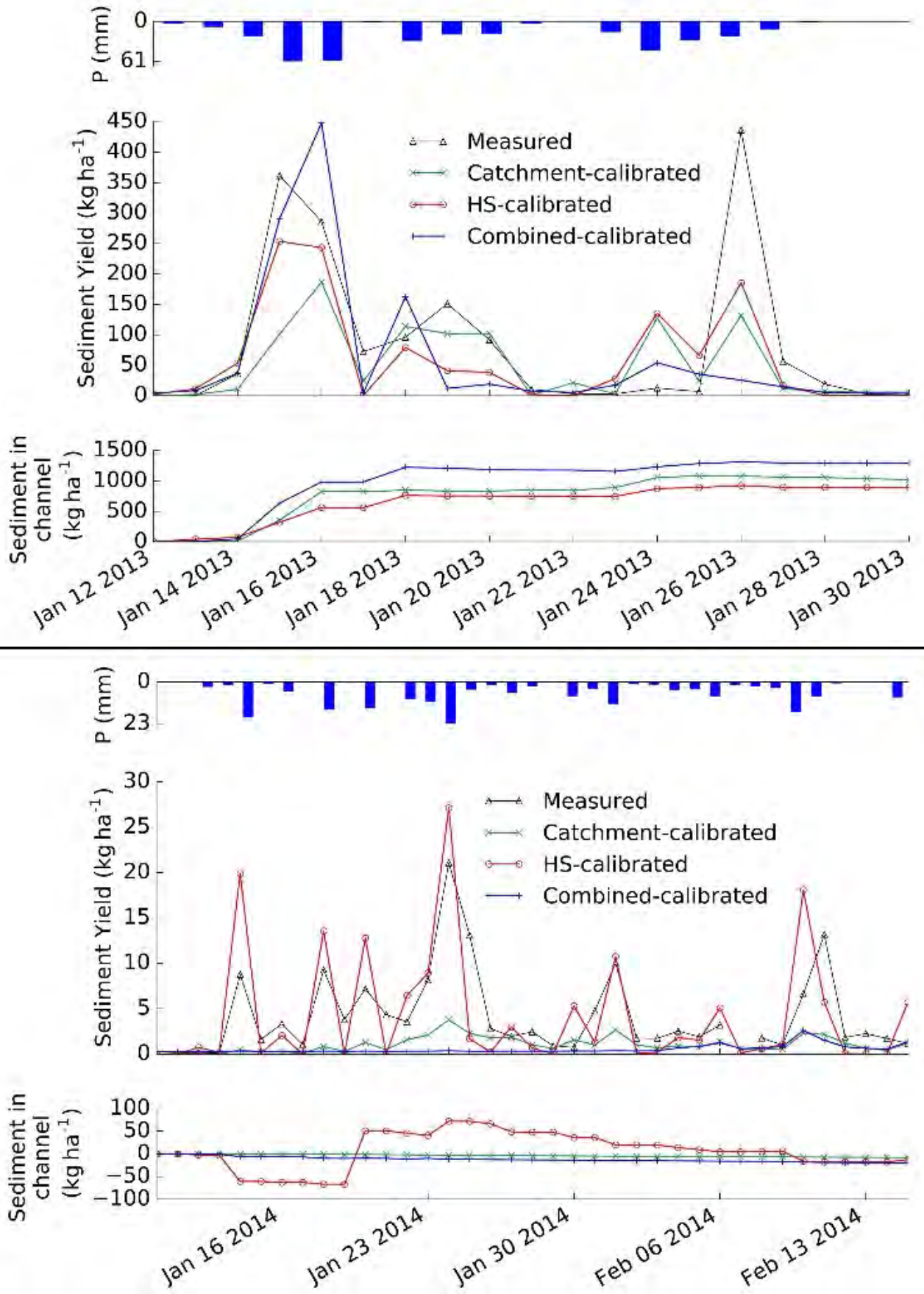

Figure 5.6. Sedigraphs of sediment output at the outlet of the catchment for two periods in 2013 and 2014 for the modelled sediment yield using the hillslope(HS)-calibrated, catchment-calibrated and combinedcalibrated model. The lower figures for both years show the amount of sediment accumulated or eroded from the channel, calculated from the start of the figure. 


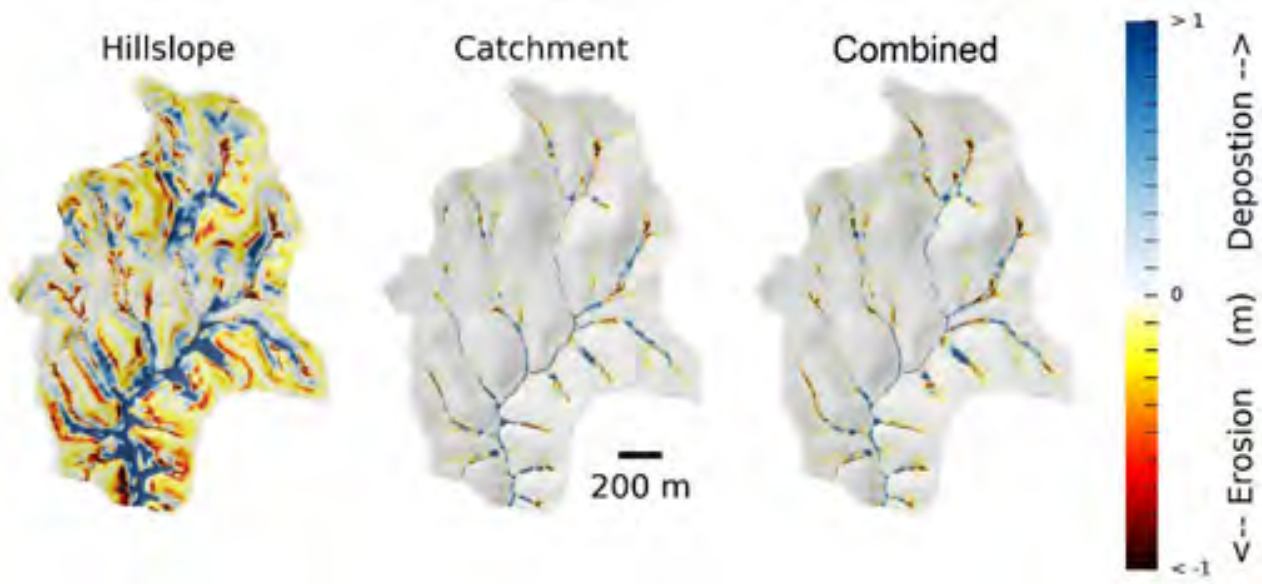

Figure 5.7 Modelled erosion and deposition using the hillslope-calibrated, catchment-calibrated and combined-calibrated models for the entire model run (4 years).

When comparing spatial erosion and deposition patterns to observed erosion features (Figure 5.8), all models show large amounts of erosion on the slopes, with much of the sediment depositing again in the valley bottoms. The observed gully is only partly modelled by the hillslope-calibrated model. The catchment-calibrated model and the combined-calibrated model do not show the gully development. The influence of the vegetation can also be seen from the spatial patterns. The vegetated areas do not erode as heavily as the agricultural areas.

The different hydrological behaviour of the three models, resulting in distinct patterns of HOF and SOF can be seen as well in the erosion and deposition patterns (Figure 5.8). These behaviours are especially visible for the year 2013-2014. The hillslope in the northeast of the area is eroded entirely in the hillslope-calibrated model (HOF along the entire slope), while for the catchment-calibrated model there is only a 1-3-pixel wide band of erosion before the deposition zone ( $\mathrm{SOF}$ at the bottom of the slope). The combined-calibrated model shows erosion higher up the slope than for the catchmentcalibrated model, which is caused by SOF already occurring mid-slope. 


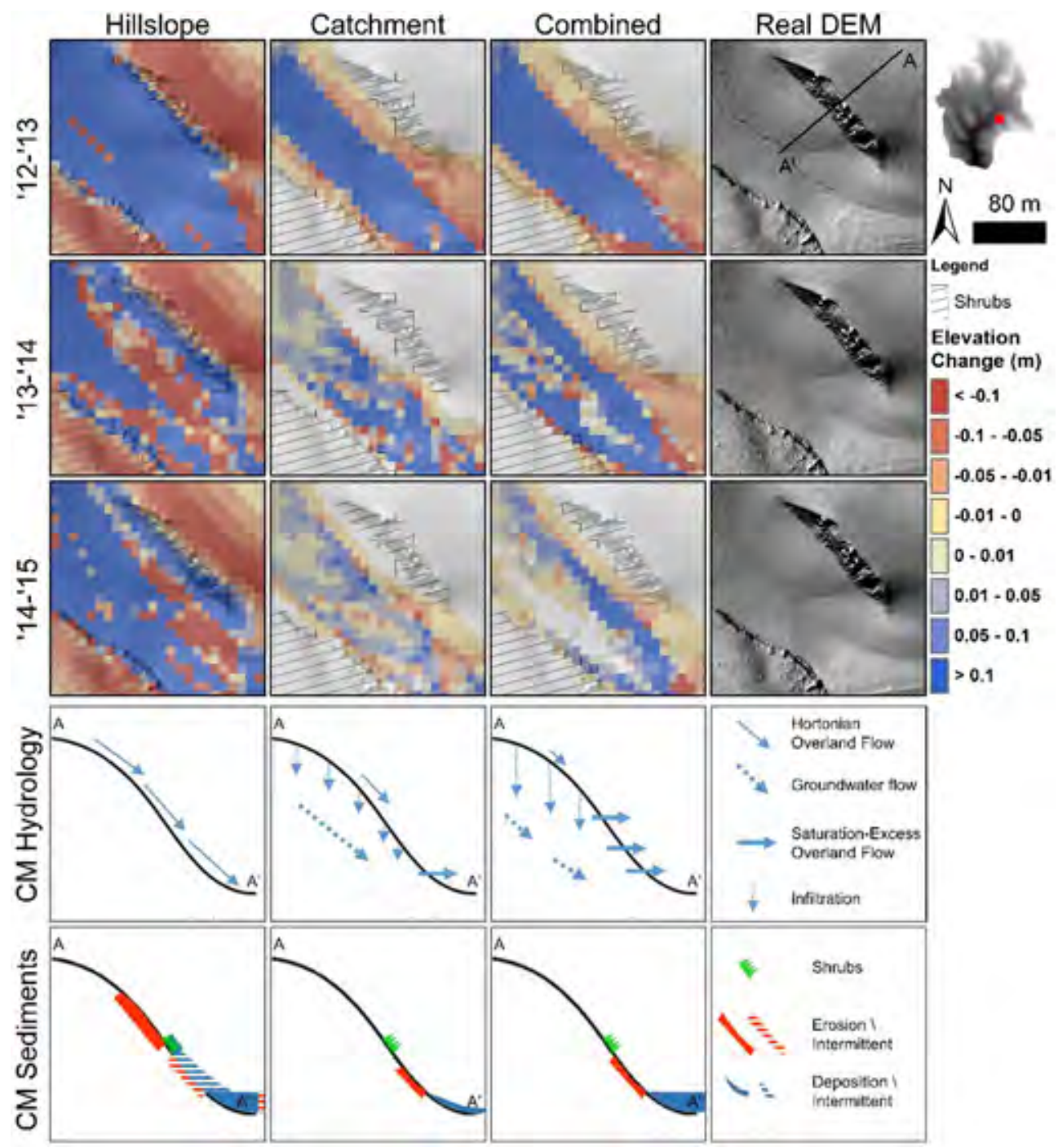

Figure 5.8 Modelled erosion and sedimentation using the hillslope-calibrated, catchment-calibrated and combined-calibrated models for three consecutive years. For all three years the real DEMs (resolution: $12 \mathrm{~cm}$ ) from stereo photogrammetry of February for each year are depicted in the last column. Two bottom rows of the figure show conceptual models (CM) of the different model processes for hydrology and erosion/deposition on a hillslope segment $A-A^{\prime}$, of which the location is indicated in the top right image. The hillslope-calibrated model is dominated by infiltration excess (Hortonian) overland flow (HOF). The catchment model is dominated by saturation-excess overland flow (SOF), mainly at the lower part of the slopes, while the SOF in the combined-calibrated model already starts mid-slope. For details on obtaining the high-resolution digital elevation models see chapter 2). 


\subsection{Discussion}

\subsubsection{Modelling water discharge}

Results of the hydrology calibration (Figure 5.4) show that optimum parameter combinations for hillslope scale and catchment scale are very different. Calibrating only with data from the hillslope scale, infiltration excess overland flow becomes the dominantly simulated mode of flow creation and this is evidenced in the hillslope data. This can be seen from the high model efficiencies at low infiltration rates. The model predictions are less sensitive to change for the throughflow parameter in these low infiltration settings, due to already limited soil water. Soil depth is important for the catchment scale model, but less so for the hillslope scale model. A shallow soil depth at hillslope scale reduces the sensitivity of the infiltration and throughflow parameters, because soil depth is already limiting infiltration, making changes in infiltration and thus, throughflow only minor. Furthermore, the hillslope-calibrated model better performs at the hillslope than the catchment-calibrated model at the catchment.

Low flow peaks were overestimated and the large peaks were underestimated (Figure 5.5). This is especially the case for the catchment-calibrated model and the combinedcalibrated model. This means that all model versions have problems with simulating the catchment groundwater storage depletion and renewal at some stage, which was also the case in the study of Keesstra et al. (2014). Furthermore, models generally tend to have problems modelling Mediterranean systems that are characterised by strong wet and dry contrasts (Serpa et al., 2015; Tavares Wahren et al., 2016). The lack of proper groundwater storage representation in the model could be improved by also simulating e.g. deep percolation to improve the model's base flow predictions. However, parameters for this deep percolation need to be determined. The deep percolation could be added using values from literature or as a calibration parameter, because it is otherwise not easily measured in a catchment - especially spatially explicit.

The validation model efficiencies (MEFs) for hydrology show that the hillslopecalibrated model is robust, with an increase in model efficiency relative to calibration, whereas the MEFs obtained with predictions from the catchment-calibrated model and combined-calibrated model decrease by about a third. The combined-calibrated model performs the best at the catchment scale for the large discharge peaks and even for the extreme events, which is also visible in the validation MEFs (Table 5.4). Modelling the peak discharges correctly is in most places the most important objective of modelling, because of the amount of sediments transported during this peak (Seeger et al., 2004; Keesstra et al., 2009b). These results show that combining measurements of runoff at two spatial scales improves the simulation of certain aspects of the hydrograph and, more importantly, could better simulate the hydrological behaviour within the catchment.

MEFs for hydrology at the catchment scale for calibration are higher than those obtained for the same catchment using a linear regression model (0.639 vs. 0.50; chapter 4; Masselink et al., 2016b), while they are both $\sim 0.4$ for the validation. The LAPSUS-D model, however, allows for looking at water and sediment movement 
spatially, something the linear regression model does not allow. Chahor et al., (2014) used the AnnAGNPS model to model hydrology and sediment yield within the same catchment on a monthly, seasonal and yearly basis, obtaining Nash-Sutcliffe model efficiencies between 0.35 and 0.79 for hydrology, which are partly higher than obtained here on a daily timestep using the LAPSUS-D model. The LAPSUS-D model, however, allows for better spatial and temporal representation of overland flow and groundwater flow.

\subsubsection{Modelling sediment discharge}

The reported MEF values for both the calibration and validation period for all three models are higher than those reported for the same catchment by Masselink et al., (2016a) using a leave-one-out cross validation for a linear regression model. They obtained a mean MEF of 0.16 for calibration and a mean MEF lower than 0 for validation. Chahor et al. (2014) reported good calibration results for the same catchment using the AnnaGNPS model at the annual scale, but unsatisfactory model results at the monthly and seasonal scales $\left(\mathrm{R}^{2} \quad 0.2-0.3\right)$. The linear regression model does not allow for analysing spatial patterns of erosion and deposition, while the AnnaGNPS model only discriminates spatially between sub-catchments.

The results of the sedigraphs show that the hillslope-calibrated model is the best out of the three models at capturing the sediment peaks, which is probably because the hillslope-calibrated model is able to properly predict overland flow at the hillslope. This then results in sufficient water availability in the hillslope-calibrated model for sediment transport, while the water availability is the limiting factor in the other two calibrated models. The hillslope-calibrated model is, furthermore, the only model that shows sediment erosion and deposition dynamics in the channel, while the other two models show mainly aggradation in the channel. In reality, the channel in the Latxaga catchment is in many places eroded down to the bedrock and channel aggradation and subsequent erosion are common. There is, however, a need to quantify these processes within the channel for the improvement of our understanding and the subsequent implementation of these processes in the model.

The initial increase of sediments in the channel (Figure 5.6; 2013) could be due to the initial conditions of the model, with no sediments present in the channel. The spin-up time of 1 year could have not been enough to fill up the channel sufficiently to reach equilibrium conditions. This spin-up time could also be one of the reasons why model efficiencies for 2012-2013 for all models are all below zero (Table 5.5). Until the channel is sufficiently filled with material there is not enough sediment discharge out of the catchment.

The overall amount of erosion and deposition in all models (Figure 5.7) is higher than observed erosion and deposition values. Especially the deposition in the valley bottoms (up to several meters for the hillslope-calibrated model) far exceeds the actual observed deposition, i.e. up to $\sim 25 \mathrm{~cm}$ at sinks at valley floors in a single year (pers. obs.). The inability of correctly representing erosion and deposition patterns in the models is most likely due to the influence of slope in the transport capacity equations. 
The changes in slope from mid-slope to foot-slope cause a sudden drop in transport capacity, which in turn causes deposition. These issues with sudden large deposition patterns can even more clearly be seen in Figure 5.8. Here, again, the hillslopecalibrated model is the only model that shows dynamics on the hillslope that resemble observed dynamics, i.e. sediments originating from most of the hillslope and gully formation such as that observed for the winter of 2012-2013. The magnitude of the dynamics is, however, severely exaggerated. Correctly modelled patterns of erosion, pathways and sinks are a first step for management purposes and, therefore, important (e.g. Hunink et al., 2012).

To counteract the large influence of slope in the transport capacity calculation, both erodibility and sedimentability in this study are up to 5 orders of magnitude higher than in other LAPSUS studies (Schoorl et al., 2002; Baartman et al., 2012a, 2013). These values in other studies, however, were used for long-term landscape evolution modelling with a yearly time step, which makes it difficult to compare them with the ones used in this study. The large changes in transport capacity due to large influence of slope could be partly resolved by changing the $n$ parameter in the transport capacity equation, or by separating hillslope sediment transport from channel sediment transport. This has been applied previously in the original LAPSUS model (Baartman et al., 2012a) and is common in other models, like CAESAR (Coulthard et al., 2002). By separating hillslope and channel sediment transport processes, the actual processes can be more accurately modelled for both hillslope and the channels. This means that for e.g. the hillslope-calibrated model the overall erosion and sedimentation will decrease, while erosion and deposition patterns will stay similar.

\subsubsection{Hydrological and Sediment Connectivity}

The implication of the mirrored image in the calibration space, and the large differences in the hydrographs for the models that were calibrated against data from different scales is that we cannot make predictions about water flow at hillslope scale when only the catchment scale data are used and vice versa. The better the calibration is at the catchment scale, the worse it will reproduce runoff at the hillslope scale for a number of calibration parameters. Optimising the model for one scale draws it away from the optimum at a different scale.

The three differently calibrated models show distinct patterns of overland flow generation and, therefore, distinct patterns of erosion and deposition (Figure 5.8). The actual hydrological behaviour within the catchment is complex and very dependent on antecedent moisture (Masselink et al., 2016). In winter, soils are saturated and even small, low intensity events produce overland flow. In summer, soils can be extremely dry and large, high intensity events do not always produce overland flow (Giménez et al., 2012b).

Both hydrograph response and hydrological connectivity could be modelled better using an approach where first hillslope-scale parameters are determined by e.g. measuring infiltration rates, and then adjusting catchment-scale parameters related to soil water saturation and channel flow (Nunes et al., 2009). For future studies, models 
such as LAPSUS-D should take these differences in hydrological behaviour into account. This can be done by introducing e.g. soil water repellency or crusting into the model, as is currently done in for example the LandSoil model (Ciampalini et al., 2012) and making unsaturated groundwater flow dependent on local soil moisture patterns (Western et al., 2001). Furthermore, a better prediction of soil depth distribution can lead to better hydrograph simulations (Tavares Wahren et al., 2016)

Almost all sediment arriving at the outlet for the catchment-calibrated model and the combined-calibrated model is sediment that is eroded and exported out of the catchment within the same day. This immediate export of sediments out of the catchment becomes visible by looking at the channel sediment dynamics (Figure 5.6), which are almost non-existent for the catchment-calibrated model and combinedcalibrated model. The hillslope-calibrated model does show channel sediment dynamics, which could represent natural patterns of bank and channel erosion and inchannel deposition (Thompson et al., 2016). In this study no data was available to verify to which extent these processes were present in the Latxaga catchment.

In order to keep connectivity in models an emerging property of the system (cf. Okin et al., 2015), processes like bank erosion need to be monitored, and proper relationships for e.g. the buffering capacity of riparian vegetation need to be established and used in the model (Keesstra et al., 2012; Poeppl et al., 2012).

\subsubsection{Towards incorporating multiple scale measurements in model calibration}

In this study, the combined-calibrated model, using the combination of the two different spatial scale datasets, performed better at the catchment scale than the catchmentcalibrated model. Furthermore, the hillslope-calibrated model showed the highest MEF for sediment discharge. These results show the importance of including measured data at multiple scales to properly model hydrological connectivity. Results from this study show that having only data at the outlet for model calibration do not give the best model results in terms of spatial patterns of erosion and deposition. Adding measured data at multiple scales decreases the chance of misrepresenting hydrological and sediment processes. In this sense, we confirm results from other studies that showed that by using data from multiple sources, the model efficiency can be improved (McMillan et al., 2011; Finger et al., 2015). The desired scale at which to calibrate the model is dependent on the objective of the study. If the goal is to assess locations for soil and water conservation measures on a hillslope, the model ideally, should be calibrated at both hillslope and catchment scale. If the objective is to model influx into a downstream reservoir, just calibrating the model at catchment scale could suffice.

Measurements of hydrology and sediment transport at multiple scales can greatly improve model predictions, even if the data are semi-quantitative, or show high variability, as long as this variability is taken into account (Nearing, 2000). We showed this here by using overland flow connectivity data. The measured hillslope runoff data are not quantities of runoff, because it is a measure for connectivity (Masselink et al., 2017) multiplied by the daily precipitation amount. However, even using these semiquantitative data, the combined-calibrated model performs better than the catchment- 
calibrated model alone. The model efficiency, the spatial patterns of erosion and deposition and the qualitative comparison of the sedigraphs were all better for the hillslope-calibrated model than for the catchment-calibrated model. The catchmentcalibrated model performed better only in terms of absolute elevation changes within the catchment. These results show that by using an erosion model of which the hydrology is calibrated by just mimicking the hydrograph, one might not end up representing the relevant processes within the model. Different combinations of parameter settings for hydrology possibly lead to similar hydrograph simulations (i.e. equifinality). These different parameter settings, in turn, affect the ability of the model to properly simulate erosion and sediment transport processes (Brazier et al., 2000; Beven, 2011).

For future modelling studies, we recommend to always use data at multiple scales when available. For the improvement of models, it is necessary that measurement campaigns are done with a combination of measurements at different scales. These measurements preferably start at small scale measurements of e.g. infiltration and erosion using rainfall simulators (Nunes et al., 2009; Rodrigo Comino et al., 2016), to (un)bounded plot erosion measurements (Cerdan et al., 2010), hillslope-channel tracer experiments (Guzmán et al., 2013) and measuring channel erosion and deposition along large parts of the reach (Milan et al., 2007). The low cost and high accuracy of digital elevation models (DEMs) from unmanned aerial vehicles and (terrestrial) laser scanning offer the possibility of using DEMs of Difference to obtain erosion and deposition quantities on hillslopes (Smith and Vericat, 2015), gullies (Giménez et al., 2009) or for bank erosion. These techniques, however, are often not suitable for agricultural areas due to the large elevation differences throughout the year due to e.g. tillage and compaction.

\subsection{Conclusions}

In this study, we applied a reduced-complexity hydrological and sediment transport model, the LAPSUS-D model, to an agricultural catchment in Northern Spain. The model was calibrated and validated for a 4-year period from 2011-2015 using three datasets at varying spatial scales: hillslope, catchment and the combined dataset (combinedcalibrated model). The three model versions calibrated against the three datasets show distinct hydrological behaviour. The hillslope-calibrated model was dominated by infiltration-excess overland flow, the catchment-calibrated model by saturation-excess overland flow at the bottom of the hillslopes and the combined-calibrated model was dominated by saturation-excess overland flow from the middle to the bottom of the slope. For hydrology, the combined-calibrated model simulated the large discharge peaks best, while at the hillslope scale, the hillslope-calibrated model performed best. The hillslope-calibrated model had the highest model efficiencies for sediments, both for calibration (0.618) and validation (0.269). The hillslope-calibrated model was also the only model that shows observed gully erosion on a high-resolution DEM and displayed channel sediment dynamics. However, absolute quantities of erosion and deposition within the catchment were too high. The results show that modellers need to be aware of problems associated with automatic calibration, over-calibration and not incorporating measured data at multiple spatial scales. We advocate incorporating 
runoff and sediment tracing data at multiple scales whenever this is possible and to carry out specific measuring campaigns towards this end. 


\section{Chapter 6}

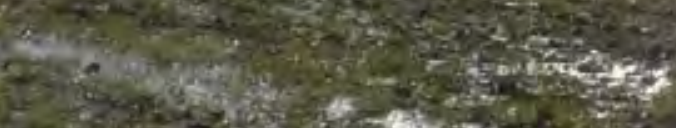

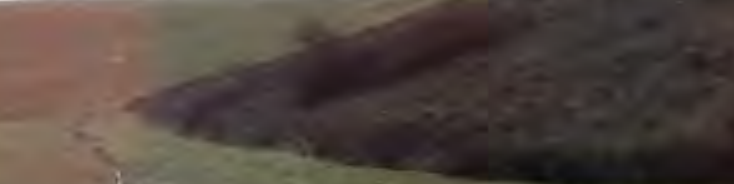

2.

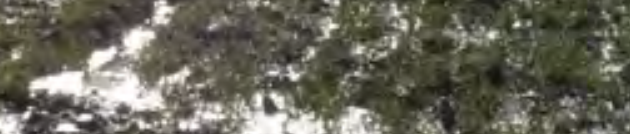

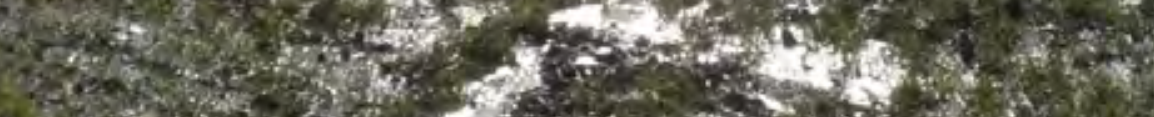

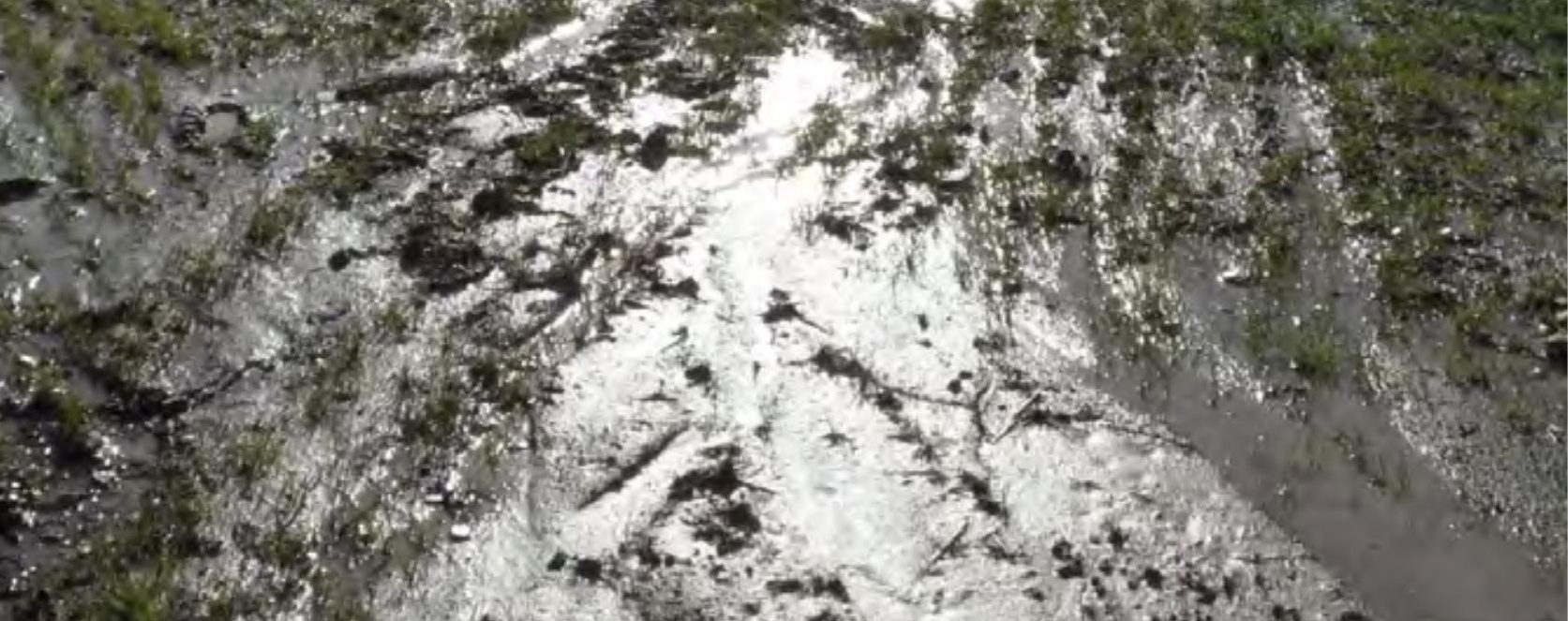




\section{Chapter 6. Synthesis}

The connectivity concept has evolved within hydrology and geomorphology over the past years for obtaining better process understanding about water, sediment and nutrient fluxes. Connectivity refers to how well a landscape facilitates or impedes the movement of water and associated substances (Pringle, 2003). Connectivity can refer to many processes, acting at multiple spatial and temporal scales. Therefore, many terms using connectivity have been mentioned in this thesis. Some examples are: hydrological connectivity, sediment connectivity, hillslope-channel connectivity, structural connectivity, functional connectivity, soil moisture connectivity and connectivity thresholds. Hydrological connectivity and sediment connectivity are overarching connectivity terms relating to water and sediment transport. Structural connectivity derives from the system's (landscape) anatomy, while functional connectivity is used to explain how water and sediment actually move over the structure. Specific terms like hillslope-channel connectivity and soil moisture connectivity refer to the connectivity of sediment and water between different compartments within the landscape or the soil.

In this thesis, hydrological and sediment connectivity were studied in humid Mediterranean systems by measuring landscape structure, water and sediment fluxes and by doing sediment tracing. Furthermore, these fluxes and consecutive changes to the structure were modelled, both spatially explicit and lumped.

\subsection{Research Questions}

To improve our understanding of hydrological and sediment transport processes, the topics of hydrological and sediment connectivity were addressed in this thesis. Efforts to investigate connectivity were done from the perspectives of measurements and models and by combining measured data at different scales with each other using, for example, networks (graph theory). The previous chapters are discussed here, in light of the posed research questions.

\section{Can networks aid in obtaining a better understanding of hydrological connectivity at the hillslope scale?}

Networks have only been used sporadically in connectivity studies (Heckmann et al., 2015), but the research community is now beginning to see the value of networks for analysing measured data and for their use in models. In chapter 2 it was shown that networks are a powerful tool for connecting data on structural connectivity with data on functional connectivity.

Networks in geomorphology are constructed using information about structure from either Digital Elevation Models (Masselink et al., 2017) or from satellite imagery (Marra et al., 2014). Network topology by itself can reveal many details about the structure and functioning of the system. If measured data of actual fluxes within the network are added, networks can become an even more powerful tool to study geomorphological processes within the connectivity concept. 
By using networks to combine high-resolution spatial data and overland flow measurements at high temporal resolution it was possible to demonstrate differences in overland flow connectivity for agricultural areas and shrub areas. Additionally, a negative correlation between connectivity and soil moisture was found. This negative correlation is counter-intuitive in normal situations, but may be caused by soil water repellency (Doerr and Moody, 2004), although this was not confirmed for this study area. Without the use of networks, it would have been more difficult to distinguish the different hydrological behaviours for the different areas, as well as to indicate important factors for overland flow connectivity.

Networks provide powerful data structures for the assessment of static representations of landscapes, but they can be even more powerful when combining measurements of structural and functional connectivity as shown in chapter 2. Networks can act as data structures for models (Heckmann and Schwanghart, 2013), and can make it easier to discern complex patterns related to water and sediment transport. It is, therefore, worth the effort to further explore the use of networks for studying connectivity.

\section{How are event characteristics linked to hillslope-channel connectivity in terms of water and sediments?}

Many studies towards the assessment of overland flow, soil erosion and event characteristics have focussed on multiple spatial and temporal levels (e.g. Boix-Fayos et al., 2006). By increasing the spatial and temporal resolution of overland flow measurements at the hillslope scale we showed that for overland flow connectivity in the Latxaga catchment, mainly antecedent precipitation and precipitation intensity are important (chapter 2). During the winter of 2014-2015, no sediment transport from the hillslope to the channel occurred in the Latxaga catchment (chapter 3). We found that absolute precipitation quantities were not lower for that particular time period, but precipitation intensity was lower than in other years. This means that for the events during that winter the hillslope was not connected to the channel in terms of sediments and that this was mainly due to low precipitation intensities.

The importance of precipitation intensity for hydrological and sediment connectivity is also shown in other studies in Mediterranean catchments (Baartman et al., 2012b; Giménez et al., 2012b). Total precipitation seems to have low influence on sediment detachment. Overland flow is generated also during lower intensity events (Masselink et al., 2017), but precipitation intensity and flow velocity are not high enough to detach sediments from the surface. For other areas, where soils are more erodible, the minimum thresholds for hillslope-channel connectivity will be lower. In other areas, where the soil is less erodible, or topography is less pronounced, the threshold for hillslope-channel connectivity will be higher (Figure 6.1).

Other than soil erodibility and precipitation intensity, hillslope configuration in terms of topography and vegetation is important for hillslope-channel connectivity. The hillslope can be disconnected from the channel through floodplains and/or riparian vegetation as in the Latxaga catchment, or the hillslopes can be directly coupled to the 
channel. These effects of different hillslope configurations were not tested in this thesis, but is something that needs further measurement and modelling efforts, besides the studies already done towards that end (Michaelides and Wainwright, 2002).

Linking event characteristics to hillslope-channel connectivity in terms of water and sediment is a complex matter and is difficult to answer with only a few studies within a single landscape. To fully answer this research question, more research is necessary to get a more complete image of how and when hillslopes are connected to adjacent channels, preferably using detailed and continuous monitoring techniques within multiple types of environments.

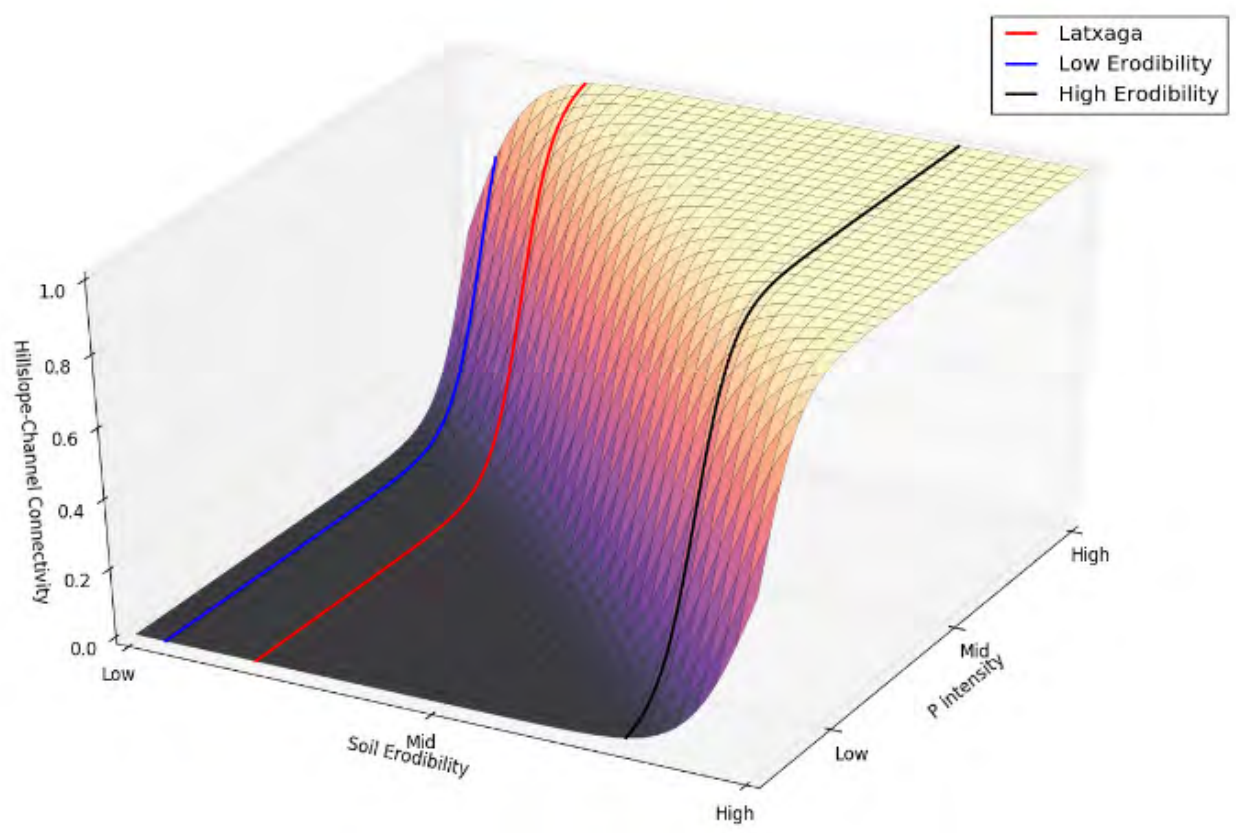

Figure 6.1 Conceptual figure for hillslope-channel sediment connectivity, based on soil erodibility and precipitation intensity. The Latxaga catchment has relatively low erodibility and needs relatively high precipitation intensity before a connection between the hillslope and the channel is established.

\section{How are water and sediment discharge of a catchment influenced by temporal changes in connectivity within a Mediterranean catchment?}

In chapter 4 the temporal changes of connectivity within three catchments were assessed by looking at changes in soil moisture and vegetation over time. Changes in soil moisture were seen as most important for the changes in hydrological and sediment 
response of the catchment. How temporal changes in patterns of saturated areas and vegetation affect the connectivity and the hydrological and sediment response of an area is a topic that needs further development as these spatially varying patterns over time were not studied in this thesis. The connections of saturated areas to the channel is something that can be explored using connectivity, using for example, networks and percolation theory (Kirkby, 2014). As with thresholds for hillslope-channel connectivity depending on topography and soil erodibility, similar thresholds for vegetation and vegetation development can be explored. Effects of vegetation and vegetation growth on hydrological and sediment connectivity can be further assessed using connectivity indices that focus on vegetation (Foerster et al., 2014). These connectivity indices, however, have been mainly derived as static indices from topography and soil surface roughness (Cavalli et al., 2013).

Linking temporal changes in connectivity to changes in catchment water and sediment discharge is a complex issue, similar to research question III. In this thesis, it was shown that soil moisture primarily controls the amount of water and sediment reaching the outlet of the catchment, which is partly in agreement with the findings about hillslopechannel connectivity (research question II; chapter 3). In chapter 3 it was also shown that antecedent precipitation was an important factor, although precipitation intensity was shown to be more important. More research on the link between temporal changes and hillslope-channel connectivity needs to be done to obtain a clearer view of the effect of temporal changes on catchment discharges.

\section{How do measured data at multiple temporal and spatial scales improve our understanding of hydrological and sediment connectivity and can these data help to improve hydrological and sediment transport models?}

In chapter 5, the LAPSUS model, a hydrological and sediment transport model, was calibrated and validated for two types of measured data: i) using water and sediment discharge data at the outlet of the Latxaga catchment, and ii) overland flow data and subsequent hydrological connectivity analyses from chapter 2 . The connectivity measurements at the hillslope scale were assumed to be a proxy for overland flow quantities. The model was calibrated at the hillslope scale (overland flow connectivity data), the catchment sale (water and sediment discharge at the outlet) and using the combined datasets. The modelling results from these three different calibrations showed large differences in system behaviour. This behaviour ranged from strongly infiltration-excess dominated runoff and erosion to strongly saturation-excess dominated. These different model behaviours show the importance of having knowledge or measurements at smaller scales than at the catchment scale. When measurements at multiple spatial scales are taken into account the modeller can be more certain of having correct process representation across all scales. By just using measured data at the outlet, the modeller risks equifinality in the model. This in turn may lead to wrong choices regarding parameter calibration.

Consequently, sediment connectivity on a hillslope, or sediment transport from the hillslope to the channel or within a catchment, can only be properly assessed from 
models by including measured data of water and sediment fluxes at multiple scales. These measurements can be done for water using traditional methods within the channel using weirs. For sediments, these methods range from taking water samples for measuring suspended sediment to sediment traps on hillslopes. Alternatively, semiquantitative data like used in this thesis, like the occurrence/absence of overland flow (chapter 2) or the connection/disconnection of sediments to the channels (chapter 3) is already valuable information that aids towards a better understanding of catchment behaviour in terms of water and sediment.

\subsection{General Discussion}

\subsubsection{The connectivity behaviour of the Latxaga catchment}

Some previous conceptual models of connectivity, for example the conceptual model of (Bracken et al., 2015) is based on sediments gradually accumulating near and in the channel, thereby increasing sediment connectivity. High sediment connectivity, in combination with a high-magnitude, low-frequency event cause large amounts of sediment to be exported out of the catchment and flush out all of the sediments, after which the cycle is repeated. From chapter 3 and 4 in this thesis it becomes clear that the Latxaga catchment is also governed by this type of sediment erosion and accumulation near the channels. However, this behaviour is notably different than suggested by Bracken et al. (2015). The behaviour of the Latxaga catchment works as follows:

I. During large events, there is a full connection from (parts of) the hillslope to the channel. Sediments are exported out of the catchment, but large quantities of sediments are deposited near and within the channels.

II. During small events, there is no connection of sediments from the hillslope to the channel and the sediments exported out of the catchment all originate from within or near the channel. These are either the sediments that were deposited during a previous large event or sediments from an occasional bank collapse.

III. Sediments are gradually removed from the channel bed during small precipitation events that follow the large event. This causes sediment availability in the channel to gradually decrease. Due to this decrease in available sediments, later small events export less sediment than events immediately after the large event. After another large event, sediments are again available in the channel and the cycle repeats.

This behaviour shows that there are large differences between the catchment sediment dynamics of the Latxaga catchment and the conceptual model of Bracken et al. (2015). Catchment sediment dynamics for other catchments, however, might behave more like the conceptual model of Bracken et al., (2015). However, most catchments likely exhibit a combination of both behaviours, with one of them being dominant, depending on e.g. morphology, vegetation and climatic conditions. For humid environments the behaviour as found in the Latxaga catchment is probably most dominant, while in semiarid environments the conceptual model of Bracken et al. (2015) might better explain catchment sediment dynamics. These large differences in behaviours highlight the 
difficulty of having a single, simple conceptual model for connectivity which is valid for all environments. Both these conceptual models could, instead, be part of a larger conceptual framework that encompasses the majority of environments and climatic settings.

\subsubsection{Connection or no Connection?}

The main objective of this thesis was, to assess and quantify hydrological and sediment connectivity in a meaningful way, which can advance our understanding of hydrological and sediment transport processes and catchment system dynamics. With the chapters in this thesis I have made a contribution to the understanding of the way humid Mediterranean systems work in terms of hydrological and sediment connectivity. Various concepts of connectivity were tested and water and sediment transport was modelled by incorporating analysed connectivity data.

Up to today, connectivity has mainly been presented in hydrology and geomorphology as concept and not as a workable model. The main reason being that connectivity cannot easily be quantified as a single number for all purposes that works across all spatial and temporal scales. Connectivity cannot be expressed that way because it can be represented by many variables. Depending on the specific research question and study area, connectivity can be expressed as e.g: 1) a volume-to breakthrough, 2) a function or 3) a probability (Hairsine et al., 2002; Croke and Hairsine, 2006; Bracken et al., 2015). Furthermore, connectivity is generally seen as an emergent property of the system (Turnbull et al., In prep). This means that connectivity emerges from multiple small-scale processes.

In chapter 3 I demonstrated that the conceptual model of Bracken et al. (2015) cannot simply be applied to the Latxaga catchment, because of different catchment sediment dynamics. The other agricultural catchment presented in this thesis (La Tejeria) has very similar properties as the Latxaga catchment in regards of climate, soil and land use characteristics, but the connectivity behaviour of the catchment is different than that of the Latxaga catchment due to differences in topography and vegetation. Hillslopes are directly connected to the channel, which has little to no riparian vegetation. The threshold for reaching hillslope-channel connections is in this case very low, causing different responses of runoff and sediment transport to the channels than observed in the Latxaga catchment for similar events (Casalí et al., 2008, 2010). Therefore, a complete overview of the functioning of a system, including connectivity, is required to increase our understanding of sediment transport dynamics.

In hydrology, geomorphology and agronomy, management practices are aimed at reducing the amount of water transported as overland flow by reducing overall lateral connectivity within landscapes. The decrease in connectivity increases soil water availability and reduces the amount of sediments transported. In ecology, however, high connectivity between different resource patches is desirable for the movement of fauna between these patches. The combination of the chapters in this thesis show that, depending of the aim of the study, various concepts of connectivity are useful. Different geologic and climatic settings cause large differences in catchment (sediment) 
dynamics. It might, therefore, not be necessary, or even possible, to strive for a single, unifying conceptual framework for connectivity. Instead, a collection of frameworks for different settings would be desirable. These frameworks could help to clarify which measurements and type of models and indices would be preferred for a particular setting.

Most stakeholders already have a natural sense for what connectivity actually is (Smetanová et al., In review), which can be used for a dialogue between research and society. From that standpoint, connectivity is useful for communication purposes between scientists, but also for communicating science to society. Using the concept of connectivity partly removes the necessity to go into the physics of sediment transport processes or other technical jargon, and at the same time facilitate better understanding of complex flow and transport processes across watersheds.

It is my honest opinion that connectivity is definitely a useful concept to advance our knowledge on water and sediment transport processes further. However, careful consideration is also required as this particular concept will not necessary provide the ultimate explanation and insights in dynamic behaviour within watersheds around the world. The gap between the different spatial and temporal scales is too complex to be bridged with a single concept like connectivity. However, the many studies about connectivity that will be published in the near future will be able to advance knowledge on water and sediment transport processes.

\subsubsection{Future Connectivity Studies}

One of the largest obstacles in current soil erosion research is moving from measurements done at a specific scale to other scales. This change in scale implies changes in dominant processes and a change in relative quantities of sediments moved within the area of interest. Connectivity as a concept was developed to be able to serve as a link to connect measurements done at different spatial and temporal scales. As of today, however, the connectivity concept has not been able to bridge this gap adequately. The conceptual frameworks that have been developed so far give no guidance on how to quantify connectivity, nor how to measure variables related to connectivity, nor how to use connectivity in models or model results. This does not mean that the connectivity concept is not useful, but it merely needs a firm step in the direction on how connectivity can be utilised to increase our process understanding for soil erosion research.

For a successful implementation of management strategies, sources, sinks and pathways need to be known spatially, and their occurrence (appearance, disappearance) needs to be known temporally (i.e. under which conditions). Several ways to assess connectivity exist, all of which can be further explored in the future. Connectivity can be described in the form of thresholds, boolean or nominal variables or as probabilities.

Connectivity thresholds can be assessed in the form of a volume-to-breakthrough (Bracken and Croke, 2007), which can, for example, be a volume of precipitation 
necessary for overland flow to begin, or a minimum precipitation intensity necessary for sufficient sediment detachment. Other possibilities for threshold descriptors can be Boolean variables (on/off), in systems where clear connected/not-connected behaviour is present. The development of these connectivity thresholds can help understand mechanisms of overland flow generation, sediment detachment and sediment transport from the hillslopes towards the channels. If certain connectivity thresholds are observed in a system, effects of management practices can be measured using these thresholds.

Other ways to describe connectivity are probabilities of connections depending on antecedent conditions and actual conditions, or a nominal value of connectivity, describing how much of the study area is connected at a given time (e.g. chapter 2; (Masselink et al., 2017). This type of approach can also be applied to other types of measurements, for example stream discharge and sediment yield. Important water and sediment contributing sub-catchments under varying conditions can be identified using this approach.

The work done in this thesis was a step towards utilising connectivity in hydrology and soil erosion research, but much work needs to be undertaken in the near future to get a better grip on hydrological and sediment connectivity. Much of these efforts need to be aimed at better frameworks, measurement strategies and analyses of indices and model results.

\section{Definition of Connectivity}

Many definitions for connectivity already exists and the large majority agrees on a definition along the line of that of an ecologist: “... connectivity is the water-mediated transport of matter, energy and organisms within or between elements of the hydrologic cycle" (Pringle, 2003). This definition alone, however, does not provide sufficient basis to effectively use it for measurements, modelling, indices and how to translate these scientific results into a tool for landscape managers. These difficulties are further increased by the fact that connectivity is an emergent property of the system, which makes explicit measuring and modelling of connectivity difficult, or even impossible (Brazier et al., In prep.; Turnbull et al., In review).

Recent conceptual frameworks that were developed also struggle with the translation of the concept and the definition into something which is readily measurable and/or usable. For future research, it is imperative that not only a clear definition of connectivity exists, but that there are also clear methods for measuring and modelling related processes and variables, translating these into connectivity, and finally, translate these connectivity results into a better process understanding or better management tools (Keesstra et al., In review).

For the coming years, theory and concept development for connectivity needs to be focused at developing methods that can improve our understanding of water and sediment fluxes through a system. In my opinion these advancements can only be done 
when the power of measured data, indices and models are combined in a meaningful way.

\section{Measuring Connectivity}

Connectivity cannot be measured directly, but has to be inferred from measurements from structure, water and sediment tracking/tracing and water and sediment fluxes (Brazier et al., In prep.; Keesstra et al., In review). All of these measurements have been used in many previous studies looking at connectivity, although no study has combined all three aspects.

Structure can be obtained from (high-resolution) digital elevation models that are created using (terrestrial) laser scanning or unmanned aerial vehicles (Westoby et al., 2012; Nadal-Romero et al., 2015). Large advances for orthophotos and digital elevation models (DEMs) have been made over the past couple of years using those techniques. DEMs of relatively large areas $\left(>1 \mathrm{~km}^{2}\right)$ can now be obtained with $\mathrm{cm}$ resolution and accuracy. With these high-resolution photographs, features that increase or decrease connectivity are more easily detected. Furthermore, repeat surveys of these kind can accurately give erosion and deposition rates over large areas. Due to the accuracy of the current DEMs, for now, these DEMs of Difference (DoDs), are limited to areas where large changes (i.e. $>\sim 5 \mathrm{~cm}$ ) have taken place or where the landscape is not affected by agricultural practices like tillage. For the coming years, however, spatial resolution will further increase, while measurement errors will decrease, making DoD methods available in more areas and for more purposes.

Water and sediment tracing and tracking has been increasingly applied for the past years. For water tracing, often the goal is to determine the various sources of the discharge of a catchment, using a variety of isotopes (Kendall and McDonnell, 2012). For sediments, a whole array of tracers exist, both naturally occurring in the soil, as well as those actively applied to the soil (Guzmán et al., 2013). These water and sediment tracer experiments have not often been combined with high-resolution structural measurements, which is where large advances can be made over the coming years. Multi-year tracking of sediments, combined with measured elevation changes from high-resolution topography can greatly increase both our understanding of catchment sediment dynamics and of connectivity. Multi-year tracking allows for determining "virtual" velocities for sediments, depending on e.g. hillslope position and antecedent conditions (Slaymaker, 2003; Wainwright et al., 2008).

Measurements of structure, in combination with water and sediment tracing can be complemented with measurements of water and sediment fluxes at multiple locations within a study area. Using these data from these three methods, sediment sources, pathways and sinks can be properly determined (Figure 6.2). A first step towards this combination was already shown for overland flow in chapter 2 , but similar efforts for sediments have yet to be undertaken.

The key to improving our understanding of sediment pathways and (temporary) sinks is to combine these three measurement types in a meaningful way. Both the structure 
and the processes acting on the structure (function) can be determined by combining these measurements. To even further increase the knowledge on water and sediment sources, pathways and sinks, these measurements need to be combined with connectivity indices and sediment transport model results. An example of how this could be done is given further below (page 102, Figure 6.2).

\section{Indices of Connectivity}

Indices of connectivity, like the Index of Connectivity (IC;(Borselli et al., 2008; Cavalli et al., 2013), have so far only been used to describe catchments, and in an occasional case to model sediment transport (López-Vicente et al., 2013b). Indices have not been used in this thesis, but for future research topics they could be helpful to couple measurements with models, to analyse model results or to allow models to run at a relatively low spatial and/or temporal resolution by using indices of connectivity as sub-grid or sub-timestep information. Because the usage of indices as model input or for analysing model results has been limited, it remains unsure whether indices can actually fulfil this role.

Measurements of structure using modern methods like (terrestrial) laser scanning and photogrammetry are often of too high resolution to be used in present day erosion models. Indices can form a step between the measurements and the model input. This can be through representing sub-grid information as a connectivity index like for example the Relative Surface Connection Function (Peñuela et al., 2015). These indices should be further expanded to not only include topography, but also measurements of vegetation, soil structure and soil moisture.

Connectivity indices are more readily applicable than spatially-explicit, deterministic models and they should be included into management tools. Indices are easily interpretable and are less data demanding than models. Indices can be combined with model results to see which areas connect to the outlet of the catchment under precipitation events with varying return periods (Figure 6.2).

\section{Modelling Connectivity}

Hydrological and sediment transport models have been used for prediction, but also for understanding catchment system dynamics. Modelling efforts should take into account that connectivity is seen as an emerging property of the system and, therefore, no connectivity should be explicitly added to the model. Instead, models should be utilised to model connections between hillslopes and streams in terms of sediment, making use of the data collected at various spatial and temporal scales, similar to what was done in chapter 5 of this thesis, but including sediment transport data at various scales as well. The only exception to not including connectivity explicitly into models could be the expression of sub-pixel variability, by using for instance a connectivity function for roughness, vegetation or an index of connectivity (Antoine et al., 2009; Peñuela et al., 2015).

The calibration of these models should not be focused at obtaining the highest possible model efficiencies for the measured data at the outlet, but instead should be calibrated 
for spatial patterns of activity of overland flow, erosion and deposition using measured spatial data like DEMs of Difference and tracers (Figure 6.2). In my view, modellers should stop altogether with calibration of models for obtaining a single parameter set, using data obtained at the outlet only. With these calibrations, there is absolutely no certainty that the model is properly representing the processes that act at the various scales. Instead, as suggested by e.g. (Beven, 2006), a range of models with varying model parameters should be accepted as possible truths.

\section{Tools for combining measurements, models and indices}

Tools are necessary to combine measurements, indices and model results of connectivity. These tools can include "traditional" analyses, using for example GIS, but also techniques that have not been used so often in geomorphology like graph theory (networks) and related theories like percolation theory (Figure 6.2).

The "traditional", but still useful, analyses that can be employed to combine measurements, model results and indices are for example GIS analyses. With these analyses, spatially explicit model results can be compared with sensor data or tracer data to compare active areas during or after precipitation events. Similarly, the connectivity between different land use areas or between hillslopes and channels can be compared using these methods, although graph theory (networks) could greatly improve these analyses.

Graph theory has not been used often in geomorphology, especially when looking at hydrological studies and erosion studies (Heckmann et al., 2015). Graph theory allows for the analysis of large amounts of data and can be particularly useful for looking at connectivity related problems (Heckmann and Schwanghart, 2013; Masselink et al., 2017). Graphs have the advantage over standard GIS techniques that connections between areas can be easily discerned and analysed. Many of the techniques and algorithms that are used in graph theory, can be used in studies looking at connectivity. Some of these techniques and algorithms include weighted network sizes (chapter 2; (Masselink et al., 2017), node contractions, number of pathways and average path lengths. Graphs can, furthermore, be used to develop hydrological models or sediment transport models, because all the necessary information on soils, vegetation, slopes, etc. can be all stored in a single graph, increasing the efficiency of model calculations. Furthermore, the resulting graphs can easily be analysed for pathways and would allow for individual water or sediment particle tracking. Other techniques involving graph theory that have already been successfully applied in other fields like social network science and ecology are for example graph motifs (Kininmonth et al., 2015), "landscape graphs" (Cantwell and Forman, 1993) and percolation theory (Hunt et al., 2014). Percolation theory deals with probability of transfer of some unit (e.g. water) from one side of a system to another, which is very relevant to what sediment transport and especially, connectivity is all about. 

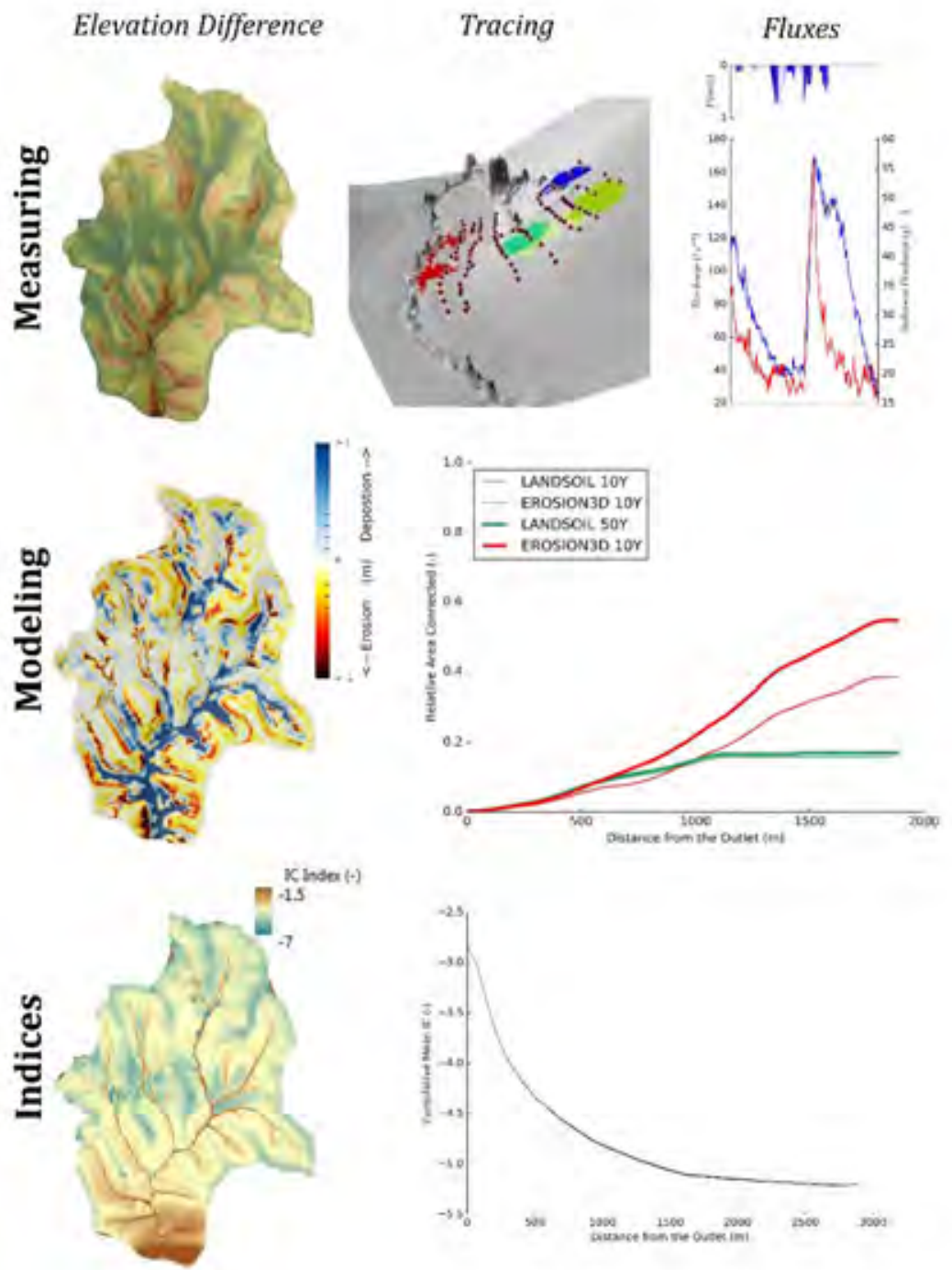

Figure 6.2. Conceptual figure on how to combine measurements, models and indices into useful connectivity results. Left page: Measuring, modelling and indices results on connectivity can be analysed first separately to look at sediment sources, sinks and pathways. Nb. The numbers and maps mentioned in the figure are largely purely hypothetical and do not represent actual measurements or other results. Figure 6.2 continues on right page. 


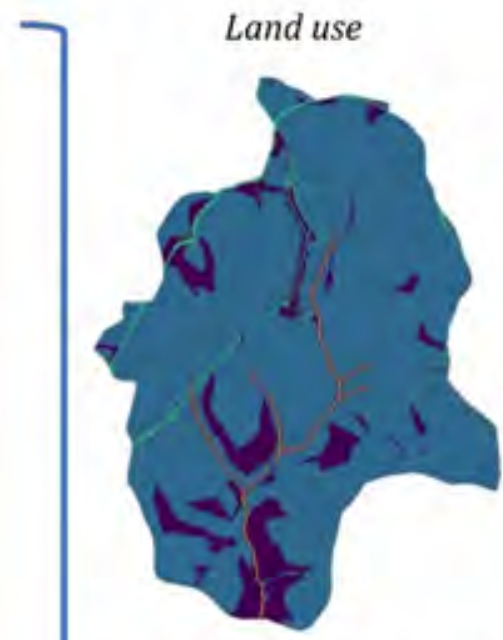

\section{Sediment transport between land use units}
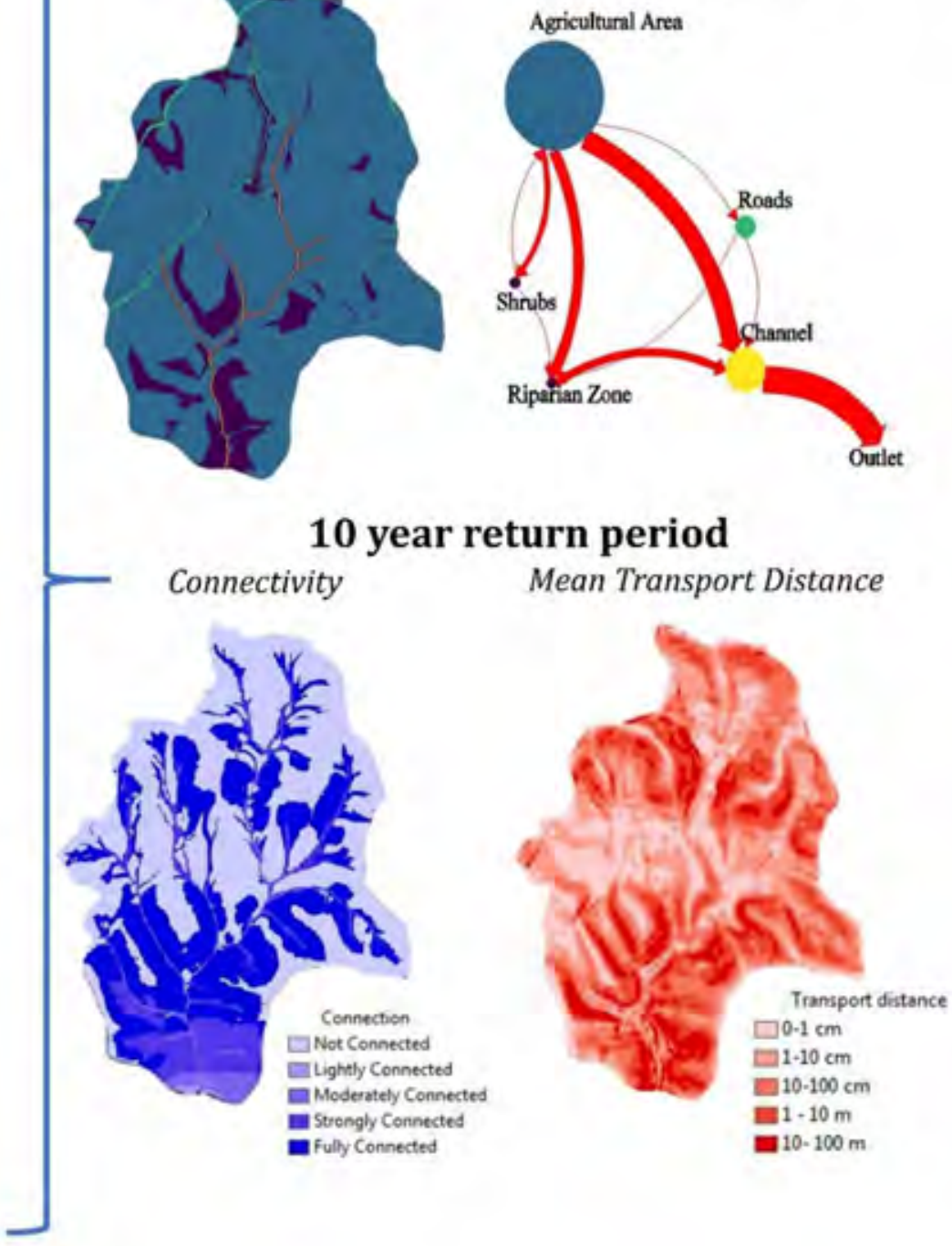

Figure 6.2 Continued. In a later stage, these results can be combined to look at sediments moving from land use units into other land use units. The size of the graph nodes indicates the relative gross erosion within each unit and the width of the graph edges indicates the amount of sediment transported to another unit (top). The results can also be analysed to look at how the different parts of the catchment are connected to the outlet during a storm with a certain return period, and what the average transport distance of detached sediment particles is (bottom). The latter two can then be combined to calculate probabilities for sediments reaching the outlet for the whole catchment. 


\section{The near and not-so-near future}

This thesis has made a considerable step towards better understanding how connectivity can be useful for i) a better understanding of hydrological and sediment transport processes at different scales, ii) the utility of the connectivity concept, and iii) setting a future research agenda on connectivity.

Whether the connectivity concept will be the magic bridge that will span the gap of scales remains to be seen. In this thesis, I already presented some insights into system functioning by looking at sediment transport issues with the connectivity concept. But even now, with the COST action on connectivity entering its fourth and final year, the research community is still partly divided on what exactly connectivity is, and how it can be used to understand processes related to hydrology, soil erosion and sediment transport. At the same time, advances in the understanding of hydrology, soil erosion and sediment transport processes are made without the use of the connectivity concept.

In order to advance our understanding of hydrological processes and sediment transport processes we need to move away from thinking how different parts in the landscape are connected, but try to see the landscape as a continuum (Bracken et al., 2015). When the landscape is seen as a continuum, we can try to approach sediment transport problems not as purely physically-based problems, but also partly stochastic, especially when using models (Aksoy and Kavvas, 2005). This view partly contradicts with the earlier mentioning of e.g. graph theory, which explicitly deals with compartments (nodes/vertices) and the connections between these compartments (edges). However, obtaining a complete view of the sediment transport continuum will take considerable effort and time, which means that as an intermediate step, the landscape still needs to be compartmentalised and graph theory will be a useful tool for this compartmentalisation.

For obtaining a complete view of the sediment transport continuum, ideally, measurements of sediment transport distances by tracking many individual particles over many years should be used to determine empirically under which conditions sediment is detached and transported. The ideal outcome would be to have for each location and setting within a watershed or hillslope, a probability of sediment detachment under certain conditions, and a probability distribution for sediment transport distance under those conditions. (Figure 6.3). These probabilities for detachment and sediment transport distance can be added to (semi-) physically based models to better deal with non-linear processes. Alternatively, completely stochastic models can be used to obtain probabilities of sediment transport distances and their confidence intervals (Lisle et al., 1998).

Obtaining these full probability distributions by using measurements, however, would need a considerable effort from many researchers for a substantial amount of years. In order to obtain sufficient data for creating probability distributions for sediment detachment and sediment transport, individual particles need to be traced and tracked for long periods of time for many types of events and antecedent conditions. This is a time-consuming process, and the large number of combinations of process variables 
make it even more challenging to test. However, lab and small plot experiments could serve as a first starting point, to explore this field further, while the gaps can be filled using models, and in the near future, Difference of Dems as well.

Ultimately, with ever increasing resolution of measurements, both spatially and temporary, and increasingly longer timeseries we are getting closer to fully understanding how sediments move within a landscape. With this increased understanding, we will be better able to provide adequate management solutions to prevent and reduce sediment transport within agricultural areas, and related substances like nutrients and pesticides. 

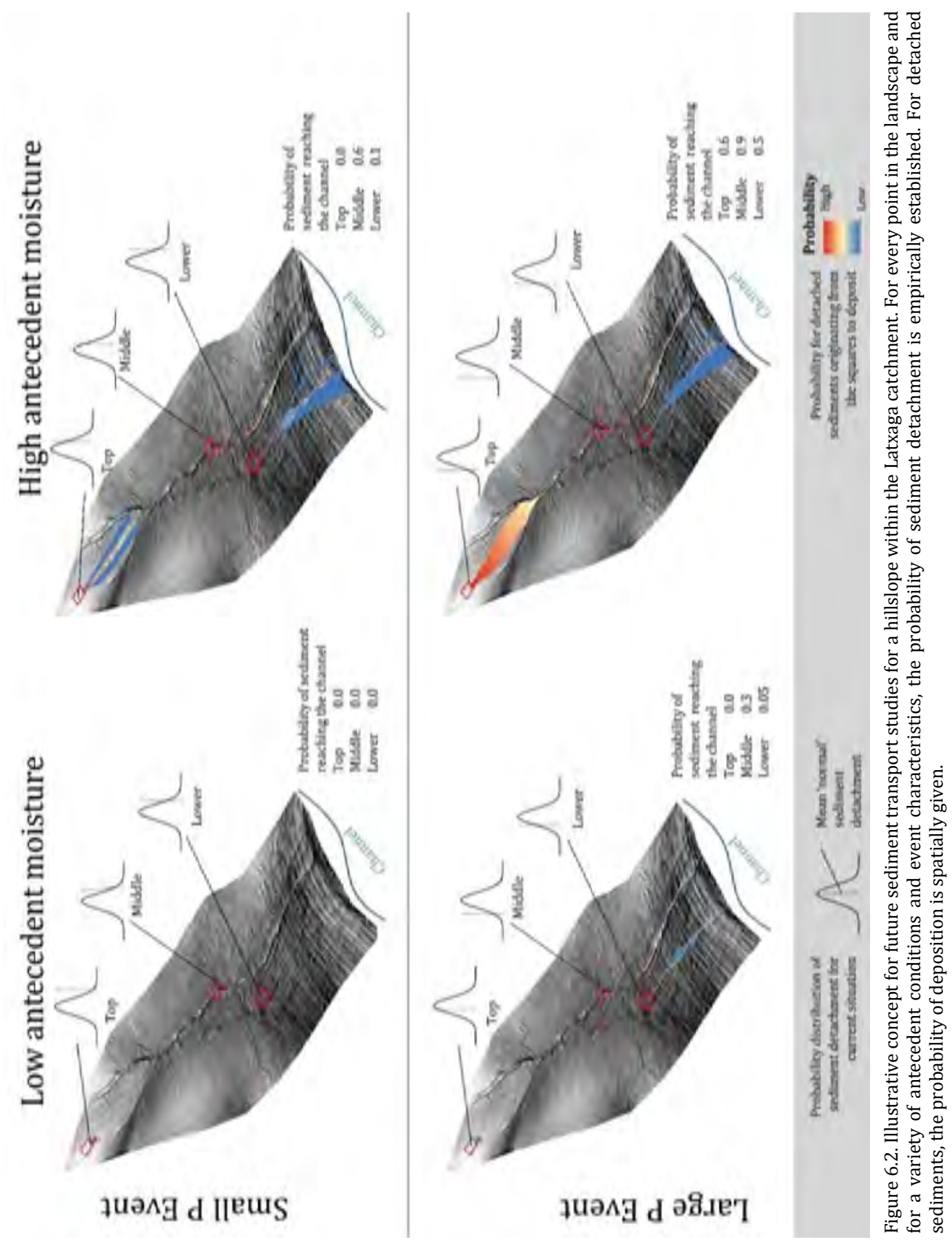


\section{Appendix A}

Supplement to chapter 2 .

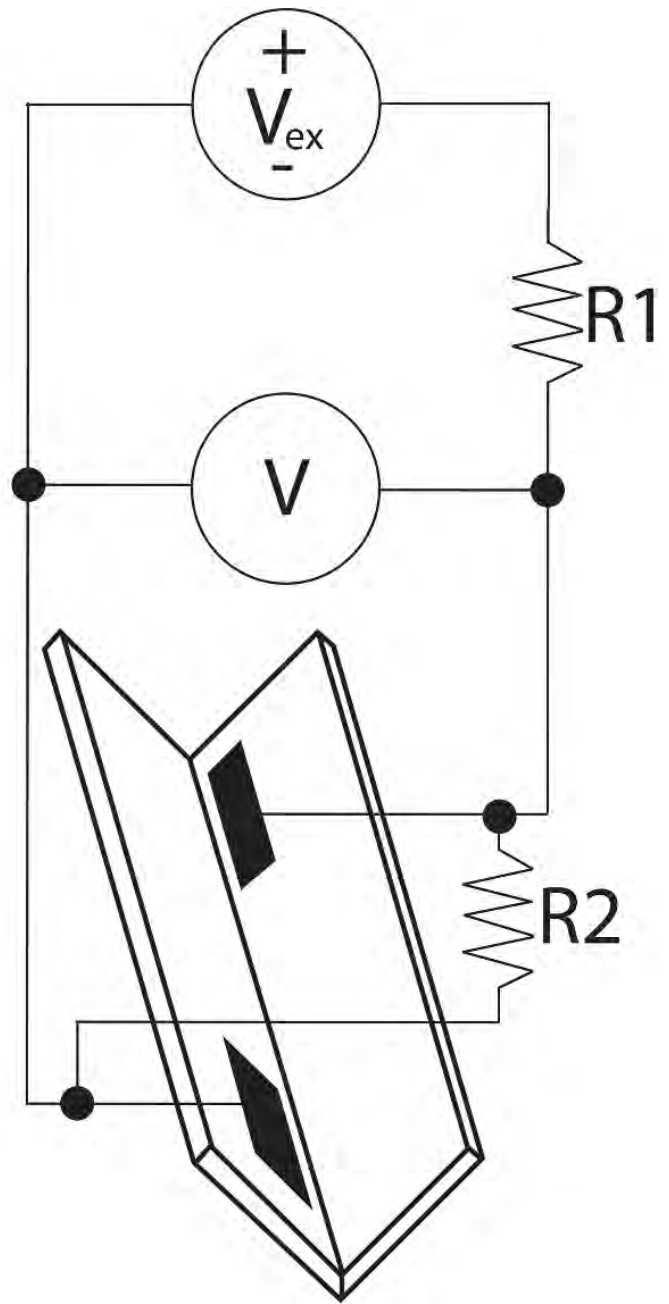

Figure A1 Electrical scheme of an overland flow sensor. $V_{\mathrm{ex}}=2.5 \mathrm{~V}, \mathrm{R} 1=100 \mathrm{k} \Omega, \mathrm{R} 2=219.2 \mathrm{k} \Omega$ 


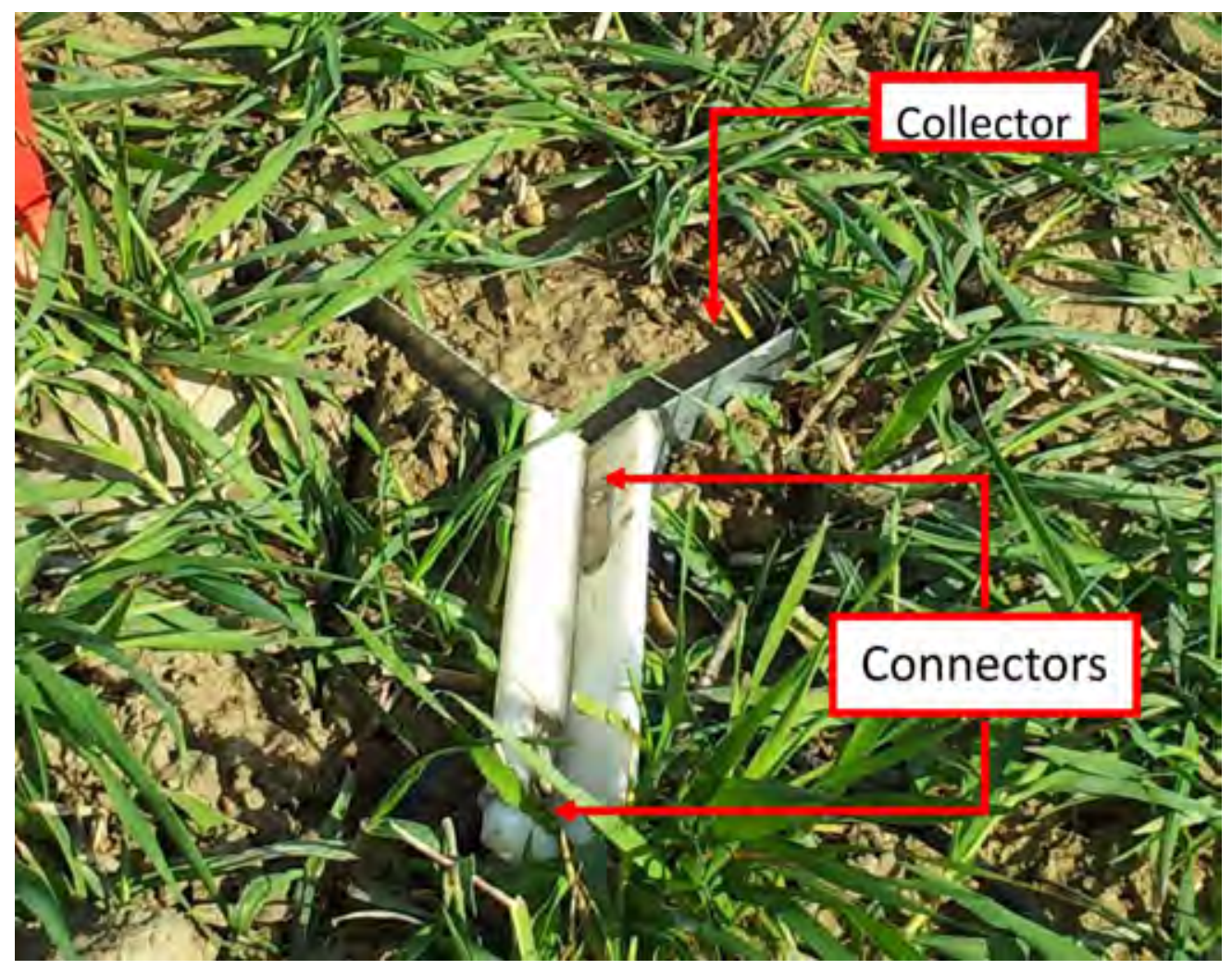

Figure A2. Photo of an overland flow sensor in the field. The roof that prevents direct precipitation entering the sensor is not depicted. 


\section{Appendix B}

Supplement to chapter 4 .

Table B1. Data, corresponding intervals and time periods for all catchments.

\begin{tabular}{lll}
\hline & Interval & Time Period \\
\hline Precipitation & $10 \mathrm{~min}$ & $1998-2014$ \\
Discharge & $10 \mathrm{~min}$ & $2002-2014$ \\
Suspended Sediment & Daily & $2002-2014$ \\
Turbidity & $10 \mathrm{~min}$ & $2002-2014$ \\
Soil moisture & $5 \mathrm{~min}$ & $03 / 13-06 / 13$ \\
Satellite Imagery (Landsat) & 16 days & $1984-2014$ \\
\hline
\end{tabular}




\section{Appendix C}

\section{Supplement to chapter 5 .}
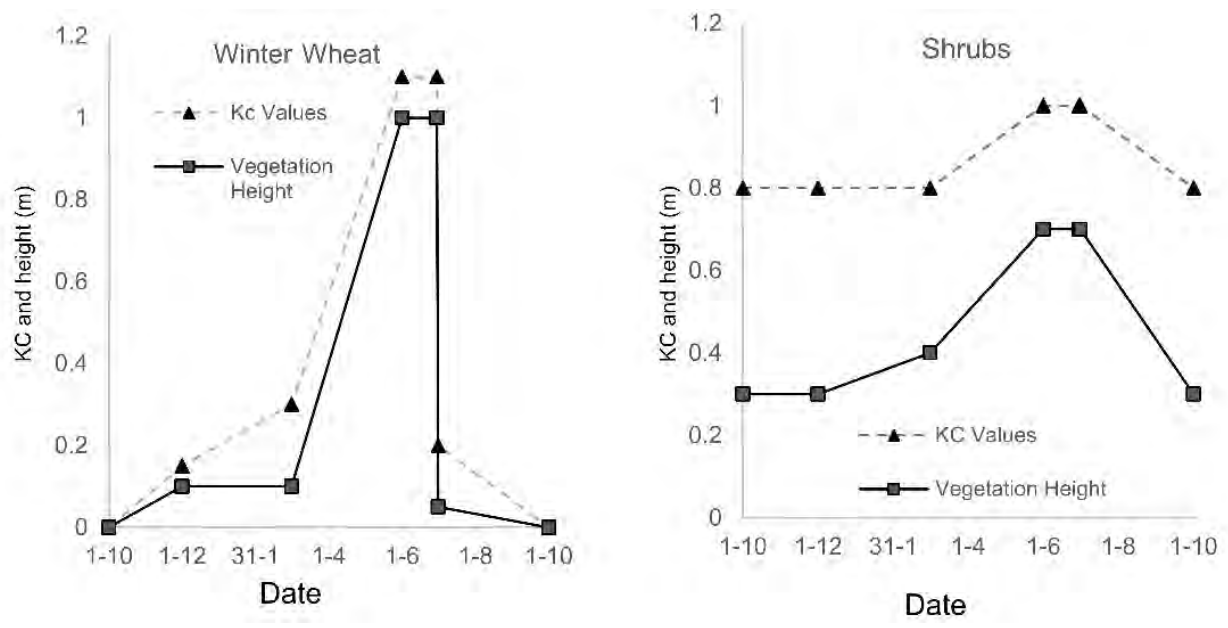

Figure C1. Used values of crop coefficients and vegetation height for winter wheat and semi-natural shrub areas for the Latxaga study area. 


\section{Bibliography}

Abrahams AD, Parsons AJ, Luk S-H. 1986. Field measurement of the velocity of overland flow using dye tracing. Earth Surface Processes and Landforms 11 (6): 653-657 DOI: 10.1002/esp.3290110608

Abrahams AD, Parsons AJ, Luk S. 1988. Hydrologic and sediment responses to simulated rainfall on desert hillslopes in southern Arizona. CATENA 15 (2): 103-117 DOI: 10.1016/03418162(88)90022-7

Aksoy H, Kavvas ML. 2005. A review of hillslope and watershed scale erosion and sediment transport models. CATENA 64 (2-3): 247-271 DOI: 10.1016/j.catena.2005.08.008

Ali GA, Roy AG. 2009. Revisiting Hydrologic Sampling Strategies for an Accurate Assessment of Hydrologic Connectivity in Humid Temperate Systems. Geography Compass 3 (1): 350-374 DOI: $10.1111 / \mathrm{j} .1749-8198.2008 .00180 . \mathrm{x}$

Ali GA, Roy AG. 2010. Shopping for hydrologically representative connectivity metrics in a humid temperate forested catchment. Water Resources Research 46 (12) DOI: 10.1029/2010WR009442

Allen RG, Pereira LS, Raes D, Smith M, others. 1998. Crop evapotranspiration-Guidelines for computing crop water requirements-FAO Irrigation and drainage paper 56. FAO, Rome 300 (9): D05109

Ambroise B. 2004. Variable'active' versus'contributing' areas or periods: a necessary distinction. Hydrological Processes 18 (6): 1149-1155 DOI: 10.1002/hyp.5536

Anctil F, Michel C, Perrin C, Andréassian V. 2004. A soil moisture index as an auxiliary ANN input for stream flow forecasting. Journal of Hydrology 286 (1-4): 155-167 DOI: 10.1016/j.jhydrol.2003.09.006

Anders NS, Masselink RJH, Seijmonsbergen AC, Keesstra SD. In prep. Ground point filtering of UAVbased photogrammetric point clouds. International Journal of Remote Sensing

Anders NS, Seijmonsbergen AC, Bouten W. 2011. Segmentation optimization and stratified objectbased analysis for semi-automated geomorphological mapping. Remote Sensing of Environment 115: 2976-2985 DOI: 10.1016/j.rse.2011.05.007

Anders NS, Seijmonsbergen AC, Bouten W. 2013. Geomorphological Change Detection Using ObjectBased Feature Extraction From Multi-Temporal LiDAR Data. IEEE Geoscience and Remote Sensing Letters 10 (6): 1587-1591 DOI: 10.1109/LGRS.2013.2262317

Antoine M, Javaux M, Bielders C. 2009. What indicators can capture runoff-relevant connectivity properties of the micro-topography at the plot scale? Advances in Water Resources 32 (8): 1297-1310 DOI: 10.1016/j.advwatres.2009.05.006

Arnold JG, Williams JR, Maidment DR. 1995. Continuous-Time Water and Sediment-Routing Model for Large Basins. Journal of Hydraulic Engineering 121 (2): 171-183 DOI: 10.1061/(ASCE)07339429(1995)121:2(171)

Baartman JEM, Gorp W, Temme AJAM, Schoorl JM. 2012a. Modelling sediment dynamics due to hillslope-river interactions: incorporating fluvial behaviour in landscape evolution model LAPSUS. Earth Surface Processes and Landforms 37 (9): 923-935 DOI: 10.1002/esp.3208

Baartman JEM, Jetten VG, Ritsema CJ, Vente J. 2012b. Exploring effects of rainfall intensity and duration on soil erosion at the catchment scale using openLISEM: Prado catchment, SE Spain. Hydrological Processes 26 (7): 1034-1049 DOI: 10.1002/hyp.8196

Baartman JEM, Masselink R, Keesstra SD, Temme AJAM. 2013. Linking landscape morphological complexity and sediment connectivity. Earth Surface Processes and Landforms 38 (12): 1457-1471 DOI: $10.1002 /$ esp.3434

Baartman JEM, Temme AJAM, Schoorl JM, Braakhekke MHA, Veldkamp TA. 2012c. Did tillage erosion play a role in millennial scale landscape development? Earth Surface Processes and Landforms 37 (15): 1615-1626 DOI: 10.1002/esp.3262 
Basher L., Ross C. 2001. Role of wheel tracks in runoff generation and erosion under vegetable production on a clay loam soil at Pukekohe, New Zealand. Soil and Tillage Research 62 (3-4): 117-130 DOI: 10.1016/S0167-1987(01)00220-3

Bautista S, Mayor ÁG, Bourakhouadar J, Bellot J. 2007. Plant Spatial Pattern Predicts Hillslope Runoff and Erosion in a Semiarid Mediterranean Landscape. Ecosystems 10 (6): 987-998 DOI: 10.1007/s10021-007-9074-3

Ben Slimane A, Raclot D, Evrard O, Sanaa M, Lefevre I, Le Bissonnais Y. 2016. Relative Contribution of Rill/Interrill and Gully/Channel Erosion to Small Reservoir Siltation in Mediterranean Environments: Catchment Erosion Processes in Mediterranean Environments. Land Degradation \& Development 27 (3): 785-797 DOI: 10.1002/ldr.2387

Bergkamp G, Cammeraat LH, Martinez-Fernandez J. 1996. Water movement and vegetation patterns on shrubland and an abandoned field in two desertification-threatened areas in Spain. Earth Surface Processes and Landforms 21 (12): 1073-1090 DOI: 10.1002/(SICI)10969837(199612)21:12<1073::AID-ESP640>3.0.C0;2-8

Beven K. 1996. 12 Equifinality and Uncertainty in Geomorphological Modelling. In The Scientific Nature of Geomorphology: Proceedings of the 27th Binghamton Symposium in Geomorphology, Held 27-29 September, 1996John Wiley \& Sons; 289.

Beven K. 2006. A manifesto for the equifinality thesis. Journal of Hydrology 320 (1-2): 18-36 DOI: 10.1016/j.jhydrol.2005.07.007

Beven K, Germann P. 1982. Macropores and water flow in soils. Water Resources Research 18 (5): 1311-1325 DOI: 10.1029/WR018i005p01311

Beven KJ. 2011. Rainfall-runoff modelling: the primer. John Wiley \& Sons.

Beven KJ, Kirkby MJ. 1979. A physically based, variable contributing area model of basin hydrology / Un modèle à base physique de zone d'appel variable de l'hydrologie du bassin versant. Hydrological Sciences Bulletin 24 (1): 43-69 DOI: 10.1080/02626667909491834

Bochet E. 2015. The fate of seeds in the soil: a review of the influence of overland flow on seed removal and its consequences for the vegetation of arid and semiarid patchy ecosystems. SOIL 1 (1): 131-146 DOI: 10.5194/soil-1-131-2015

Boix-Fayos C, Martínez-Mena M, Arnau-Rosalén E, Calvo-Cases A, Castillo V, Albaladejo J. 2006. Measuring soil erosion by field plots: Understanding the sources of variation. Earth-Science Reviews 78 (3-4): 267-285 DOI: 10.1016/j.earscirev.2006.05.005

Borselli L, Cassi P, Torri D. 2008. Prolegomena to sediment and flow connectivity in the landscape: A GIS and field numerical assessment. Catena 75: 268-277 DOI: 10.1016/j.catena.2008.07.006

Bracken LJ, Croke J. 2007. The concept of hydrological connectivity and its contribution to understanding runoff-dominated geomorphic systems. Hydrological Processes 21 (13): 1749-1763

Bracken LJ, Turnbull L, Wainwright J, Bogaart P. 2015. Sediment connectivity: a framework for understanding sediment transfer at multiple scales: Earth Surface Processes and Landforms 40 (2): 177-188 DOI: 10.1002/esp.3635

Bracken LJ, Wainwright J, Ali GA, Tetzlaff D, Smith MW, Reaney SM, Roy AG. 2013. Concepts of hydrological connectivity: Research approaches, pathways and future agendas. Earth-Science Reviews 119: 17-34 DOI: 10.1016/j.earscirev.2013.02.001

Brazier RE, Beven KJ, Anthony SG, Rowan JS. 2001. Implications of model uncertainty for the mapping of hillslope-scale soil erosion predictions. Earth Surface Processes and Landforms 26 (12): 1333-1352 DOI: 10.1002 /esp.266

Brazier RE, Beven KJ, Freer J, Rowan JS. 2000. Equifinality and uncertainty in physically based soil erosion models: application of the GLUE methodology to WEPP-the Water Erosion Prediction Project-for sites in the UK and USA. Earth Surface Processes and Landforms 25 (8): 825-845 DOI: 10.1002/1096-9837(200008)25:8<825::AID-ESP101>3.0.CO;2-3 
Brazier RE, Vericat D, Keesstra SD, Masselink RJH, Cerdà A, Blake WH, Brardinoni F, Wittenberg L, Estrany J, Nadal-Romero E, et al. In prep. Can we measure connectivity?

Breiman L. 2001. Random forests. Machine learning 45 (1): 5-32

Bren L, Turner A. 1979. Overland flow on a steep, forested infiltrating slope. Australian Journal of Soil Research 17 (1): 43 DOI: 10.1071/SR9790043

Brocca L, Melone F, Moramarco T. 2008. On the estimation of antecedent wetness conditions in rainfallrunoff modelling. Hydrological Processes 22 (5): 629-642 DOI: 10.1002/hyp.6629

Brunsden D. 1993. The persistence of landforms. Zeitschrift für Geomorphologie Supplementband 93: 13-28

Bryan RB, Poesen J. 1989. Laboratory experiments on the influence of slope length on runoff, percolation and rill development. Earth Surface Processes and Landforms 14 (3): 211-231 DOI: $10.1002 /$ esp.3290140304

Buchanan B, Easton ZM, Schneider R, Walter MT. 2012. Incorporating Variable Source Area Hydrology into a Spatially Distributed Direct Runoff Model1. JAWRA Journal of the American Water Resources Association 48 (1): 43-60 DOI: 10.1111/j.1752-1688.2011.00594.x

Bull L., Kirkby M., Shannon J, Hooke J. 2000. The impact of rainstorms on floods in ephemeral channels in southeast Spain. CATENA 38 (3): 191-209 DOI: 10.1016/S0341-8162(99)00071-5

Bunn A., Urban D., Keitt T. 2000. Landscape connectivity: A conservation application of graph theory. Journal of Environmental Management 59 (4): 265-278 DOI: 10.1006/jema.2000.0373

Bunte K, Poesen J. 1993. Effects of rock fragment covers on erosion and transport of noncohesive sediment by shallow overland flow. Water Resources Research 29 (5): 1415-1424 DOI: 10.1029/92WR02706

Butzen V, Seeger M, Casper M. 2011. Spatial pattern and temporal variability of runoff processes in Mediterranean Mountain environments - a case study of the Central Spanish Pyrenees. Zeitschrift für Geomorphologie, Supplementary Issues 55 (3): 25-48 DOI: 10.1127/03728854/2011/0055S3-0050

Cammeraat ELH. 2004. Scale dependent thresholds in hydrological and erosion response of a semi-arid catchment in southeast Spain. Agriculture, Ecosystems \& Environment 104 (2): 317-332 DOI: 10.1016/j.agee.2004.01.032

Cammeraat LH. 2002. A review of two strongly contrasting geomorphological systems within the context of scale. Earth Surface Processes and Landforms 27 (11): 1201-1222 DOI: $10.1002 /$ esp. 421

Cantón Y, Domingo F, Solé-Benet A, Puigdefábregas J. 2001. Hydrological and erosion response of a badlands system in semiarid SE Spain. Journal of Hydrology 252 (1-4): 65-84 DOI: 10.1016/S0022-1694(01)00450-4

Cantwell MD, Forman RTT. 1993. Landscape graphs: Ecological modeling with graph theory to detect configurations common to diverse landscapes. Landscape Ecology 8 (4): 239-255 DOI: $10.1007 / B F 00125131$

Casalí J, Gastesi R, Álvarez-Mozos J, De Santisteban LM, Del Valle de Lersundi J, Giménez R, Larrañaga A, Goñi M, Agirre U, Campo MA, et al. 2008. Runoff, erosion and water quality of agricultural watersheds in central Navarre (Spain). Agricultural Water Management 95: 1111-1128 DOI: 10.1016/j.agwat.2008.06.013

Casalí J, Giménez R, Díez J, Álvarez-Mozos J, Del Valle de Lersundi J, Goñi M, Campo MA, Chahor Y, Gastesi R, López J. 2010. Sediment production and water quality of watersheds with contrasting land use in Navarre (Spain). Agricultural Water Management 97 (10): 16831694 DOI: $10.1016 /$ j.agwat.2010.05.024

Cavalli M, Trevisani S, Comiti F, Marchi L. 2013. Geomorphometric assessment of spatial sediment connectivity in small Alpine catchments. Geomorphology 188: 31-41 DOI: 10.1016/j.geomorph.2012.05.007 
Cerdà A. 1997. The effect of patchy distribution of Stipa tenacissima L. on runoff and erosion. Journal of Arid Environments 36 (1): 37-51 DOI: 10.1006/jare.1995.0198

Cerdá A. 2001. Effects of rock fragment cover on soil infiltration, interrill runoff and erosion. European Journal of Soil Science 52 (1): 59-68 DOI: 10.1046/j.1365-2389.2001.00354.x

Cerdà A, Doerr SH. 2010. The effect of ant mounds on overland flow and soil erodibility following a wildfire in eastern Spain. Ecohydrology 3 (4): 392-401 DOI: 10.1002/eco.147

Cerdà A, García-Fayos P. 1997. The influence of slope angle on sediment, water and seed losses on badland landscapes. Geomorphology 18 (2): 77-90 DOI: 10.1016/S0169-555X(96)00019-0

Cerdà A, Flanagan DC, le Bissonnais Y, Boardman J. 2009. Soil erosion and agriculture. Soil and Tillage Research 106 (1): 107-108 D0I: 10.1016/j.still.2009.10.006

Cerdà A, González-Pelayo 0, Giménez-Morera A, Jordán A, Pereira P, Novara A, Brevik EC, Prosdocimi M, Mahmoodabadi M, Keesstra S, et al. 2016. The use of barley straw residues to avoid high erosion and runoff rates on persimmon plantations in Eastern Spain under low frequencyhigh magnitude simulated rainfall events. Soil Res. 54 (2) DOI: 10.1071/SR15092

Cerdan 0, Govers G, Le Bissonnais Y, Van Oost K, Poesen J, Saby N, Gobin A, Vacca A, Quinton J, Auerswald K, et al. 2010. Rates and spatial variations of soil erosion in Europe: A study based on erosion plot data. Geomorphology 122 (1-2): 167-177 DOI: 10.1016/j.geomorph.2010.06.011

Chahor Y, Casalí J, Giménez R, Bingner RL, Campo MA, Goñi M. 2014. Evaluation of the AnnAGNPS model for predicting runoff and sediment yield in a small Mediterranean agricultural watershed in Navarre (Spain). Agricultural Water Management 134: 24-37 DOI: 10.1016/j.agwat.2013.11.014

Ciampalini R, Follain S, Le Bissonnais Y. 2012. LandSoil: A model for analysing the impact of erosion on agricultural landscape evolution. Geomorphology 175-176: 25-37 DOI: 10.1016/j.geomorph.2012.06.014

Claessens L, Schoorl JM, Verburg PH, Geraedts L, Veldkamp A. 2009. Modelling interactions and feedback mechanisms between land use change and landscape processes. Agriculture, Ecosystems \& Environment 129 (1-3): 157-170 DOI: 10.1016/j.agee.2008.08.008

Clary WP, Leininger WC. 2000. Stubble Height as a Tool for Management of Riparian Areas. Journal of Range Management 53 (6): 562-573 DOI: $10.2307 / 4003148$

Cohen J. 1992. A power primer. Psychological Bulletin 112 (1): 155-159 DOI: 10.1037/00332909.112.1.155

Collins AL, Davison PS. 2009. Mitigating sediment delivery to watercourses during the salmonid spawning season: Potential effects of delayed wheelings and cover crops in a chalk catchment, southern England. International Journal of River Basin Management 7 (3): 209220 DOI: $10.1080 / 15715124.2009 .9635384$

Correll DL. 2005. Principles of planning and establishment of buffer zones. Ecological Engineering 24 (5): 433-439 DOI: 10.1016/j.ecoleng.2005.01.007

Coulthard TJ, Van De Wiel MJ. 2016. Modelling long term basin scale sediment connectivity, driven by spatial land use changes. Geomorphology DOI: 10.1016/j.geomorph.2016.05.027

Coulthard TJ, Hancock GR, Lowry JBC. 2012. Modelling soil erosion with a downscaled landscape evolution model: Earth Surface Processes and Landforms 37 (10): 1046-1055 DOI: 10.1002/esp.3226

Coulthard TJ, Macklin MG, Kirkby MJ. 2002. A cellular model of Holocene upland river basin and alluvial fan evolution. Earth Surface Processes and Landforms 27 (3): 269-288 DOI: 10.1002/esp.318

Creed I, Band L. 1998. Exploring functional similarity in the export of nitrate- $\mathrm{N}$ from forested catchments: a mechanistic modeling approach. Water Resources Research 34 (11): 30793093 
Croke J, Mockler S, Fogarty P, Takken I. 2005. Sediment concentration changes in runoff pathways from a forest road network and the resultant spatial pattern of catchment connectivity. Geomorphology 68 (3-4): 257-268 DOI: 10.1016/j.geomorph.2004.11.020

Croke JC, Hairsine PB. 2006. Sediment delivery in managed forests: a review. Environmental Reviews 14 (1): 59-87 DOI: $10.1139 / \mathrm{a} 05-016$

Czuba JA, Foufoula-Georgiou E. 2014. A network-based framework for identifying potential synchronizations and amplifications of sediment delivery in river basins. Water Resources Research 50 (5): 3826-3851 DOI: 10.1002/2013WR014227

Darboux F, Davy P, Gascuel-Odoux C, Huang C. 2002. Evolution of soil surface roughness and flowpath connectivity in overland flow experiments. CATENA 46 (2-3): 125-139 DOI: 10.1016/S0341-8162(01)00162-X

Deasy C, Quinton JN. 2010. Use of rare earth oxides as tracers to identify sediment source areas for agricultural hillslopes. Solid Earth 1 (1): 111-118 DOI: 10.5194/se-1-111-2010

Doerr SH, Moody JA. 2004. Hydrological effects of soil water repellency: on spatial and temporal uncertainties. Hydrological Processes 18 (4): 829-832 DOI: 10.1002/hyp.5518

Dunne T, Black RD. 1970. Partial Area Contributions to Storm Runoff in a Small New England Watershed. Water Resources Research 6 (5): 1296-1311 DOI: 10.1029/WR006i005p01296

Ellison W. 1944. Studies of raindrop erosion. Agricultural Engineering 25 (4): 131-136

van den Elsen HGM, Ritsema CJ, Seeger M, Keesstra SD. 2014. Averaging Performance of Capacitance and Time Domain Reflectometry Sensors in Nonuniform Wetted Sand Profiles. Vadose Zone Journal 13 (12) DOI: 10.2136/vzj2014.03.0025

Endreny TA, Wood EF. 2003. Maximizing spatial congruence of observed and DEM-delineated overland flow networks. International Journal of Geographical Information Science 17 (7): 699-713 DOI: $10.1080 / 1365881031000135483$

Esteves M, Faucher X, Galle S, Vauclin M. 2000. Overland flow and infiltration modelling for small plots during unsteady rain: numerical results versus observed values. Journal of Hydrology 228 (3-4): 265-282 DOI: 10.1016/S0022-1694(00)00155-4

Estrany J, López-Tarazón JA, Smith HG. 2015. Wildfire effects on suspended sediment delivery quantified using fallout radionuclide tracers in a Mediterranean catchment: downstream extent of fine sediment delivery from burnt headwaters. Land Degradation \& Development DOI: $10.1002 / \mathrm{ddr} .2462$

Feller W. 1968. An introduction to probability theory and its applications: volume I. John Wiley \& Sons London-New York-Sydney-Toronto.

Finger D, Vis M, Huss M, Seibert J. 2015. The value of multiple data set calibration versus model complexity for improving the performance of hydrological models in mountain catchments. Water Resources Research 51 (4): 1939-1958 DOI: 10.1002/2014WR015712

Foerster S, Wilczok C, Brosinsky A, Segl K. 2014. Assessment of sediment connectivity from vegetation cover and topography using remotely sensed data in a dryland catchment in the Spanish Pyrenees. Journal of Soils and Sediments 14 (12): 1982-2000 DOI: 10.1007/s11368-0140992-3

Foster IDL, Rowntree KM, Boardman J, Mighall TM. 2012. Changing sediment yield and sediment dynamics in the Karoo Uplands, South Africa; post-european impacts: changing sediment yield and sediment dynamics. Land Degradation \& Development 23 (6): 508-522 DOI: $10.1002 / \mathrm{ldr} .2180$

Francke T, López-Tarazón JA, Schröder B. 2008. Estimation of suspended sediment concentration and yield using linear models, random forests and quantile regression forests. Hydrological Processes 22 (25): 4892-4904 DOI: 10.1002/hyp.7110

Freeman TG. 1991. Calculating catchment area with divergent flow based on a regular grid. Computers \& Geosciences 17 (3): 413-422 DOI: 10.1016/0098-3004(91)90048-I 
Fryirs K. 2012. (Dis)Connectivity in catchment sediment cascades: a fresh look at the sediment delivery problem. Earth Surface Processes and Landforms 38 (1): 30-46 DOI: 10.1002/esp.3242

Garcia-Estringana P, Alonso-Blázquez N, Alegre J. 2010. Water storage capacity, stemflow and water funneling in Mediterranean shrubs. Journal of Hydrology 389 (3-4): 363-372 DOI: 10.1016/j.jhydrol.2010.06.017

García-Orenes F, Cerdà A, Mataix-Solera J, Guerrero C, Bodí MB, Arcenegui V, Zornoza R, Sempere JG. 2009. Effects of agricultural management on surface soil properties and soil-water losses in eastern Spain. Soil and Tillage Research 106 (1): 117-123 DOI: 10.1016/j.still.2009.06.002

García-Ruiz JM, Arnáez J, Beguería S, Seeger M, Martí-Bono C, Regüés D, Lana-Renault N, White S. 2005. Runoff generation in an intensively disturbed, abandoned farmland catchment, Central Spanish Pyrenees. CATENA 59 (1): 79-92 DOI: 10.1016/j.catena.2004.05.006

Gascuel-Odoux C, Aurousseau P, Doray T, Squividant H, Macary F, Uny D, Grimaldi C. 2011. Incorporating landscape features to obtain an object-oriented landscape drainage network representing the connectivity of surface flow pathways over rural catchments. Hydrological Processes 25 (23): 3625-3636 DOI: 10.1002/hyp.8089

Gibbs HK, Salmon JM. 2015. Mapping the world's degraded lands. Applied Geography 57: 12-21 DOI: 10.1016/j.apgeog.2014.11.024

Giménez R, Casalí J, Díez J. 2012a. Evaluación de la Producción de sedimentos y calidad de las aguas en cuencas agrarias de Navarra. Cuadernos de Investigación Geográfica 38 (2): 107-121

Giménez R, Casalí J, Grande I, Díez J, Campo MA, Álvarez-Mozos J, Goñi M. 2012b. Factors controlling sediment export in a small agricultural watershed in Navarre (Spain). Agricultural Water Management 110: 1-8 DOI: 10.1016/j.agwat.2012.03.007

Giménez R, Marzolff I, Campo MA, Seeger M, Ries JB, Casalí J, Álvarez-Mozos J. 2009. Accuracy of highresolution photogrammetric measurements of gullies with contrasting morphology. Earth Surface Processes and Landforms 34 (14): 1915-1926 DOI: 10.1002/esp.1868

Glymph L. 1954. Studies of sediment yields from watersheds. Int. Union of Geod. and Geophys., Int. Assoc. Hydrol. 10th Gen. Assem., Rome, Italy, Part 1: 178-191

Gobierno de Navarra. 1997. Mapa geológico de Navarra

Gobierno de Navarra. 2001. Estudio Agroclimático de Navarra (CD). Gobierno de Navarra, Departamento de Agricultura, Ganadería y Alimentación. Servicio de Estructuras Agrarias. Pamplona, España

Gobierno De Navarra. 2014. Digital Elevation model of Navarra Available at: http://sitna.navarra.es

Godsey S, Elsenbeer H, Stallard R. 2004. Overland flow generation in two lithologically distinct rainforest catchments. Journal of Hydrology 295 (1-4): 276-290 DOI: 10.1016/j.jhydrol.2004.03.014

Gómez-Plaza A, Martínez-Mena M, Albaladejo J, Castillo V. 2001. Factors regulating spatial distribution of soil water content in small semiarid catchments. Journal of Hydrology 253 (1-4): 211-226 DOI: 10.1016/S0022-1694(01)00483-8

Goulsbra CS, Lindsay JB, Evans MG. 2009. A new approach to the application of electrical resistance sensors to measuring the onset of ephemeral streamflow in wetland environments: Technical note. Water Resources Research 45 (9) DOI: 10.1029/2009WR007789

Gumiere SJ, Le-Bissonais Y, Raclot D, Cheviron B. 2011. Vegetated filter effects on sedimentological connectivity of agricultural catchments in erosion modelling: A review. Earth Surface Processes and Landforms 36: 3-19 DOI: 10.1002/esp.2042

Guzmán G, Quinton JN, Nearing MA, Mabit L, Gómez JA. 2013. Sediment tracers in water erosion studies: current approaches and challenges. Journal of Soils and Sediments 13 (4): 816-833 DOI: 10.1007/s11368-013-0659-5

Hairsine PB, Croke JC, Mathews H, Fogarty P, Mockler SP. 2002. Modelling plumes of overland flow from logging tracks. Hydrological Processes 16 (12): 2311-2327 DOI: 10.1002/hyp.1002 
Harvey AM. 2001. Coupling between hillslopes and channels in upland fluvial systems: implications for landscape sensitivity, illustrated from the Howgill Fells, northwest England. CATENA 42 (24): 225-250 DOI: 10.1016/S0341-8162(00)00139-9

Heathwaite AL, Quinn PF, Hewett CJM. 2005. Modelling and managing critical source areas of diffuse pollution from agricultural land using flow connectivity simulation. Journal of Hydrology $\mathbf{3 0 4}$ (1-4): 446-461 DOI: 10.1016/j.jhydrol.2004.07.043

Heckmann T, Schwanghart W. 2013. Geomorphic coupling and sediment connectivity in an alpine catchment — Exploring sediment cascades using graph theory. Geomorphology 182: 89-103 DOI: $10.1016 /$ j.geomorph.2012.10.033

Heckmann T, Schwanghart W, Phillips JD. 2015. Graph theory - recent developments of its application in geomorphology. Geomorphology 243: 130-146 DOI: 10.1016/j.geomorph.2014.12.024

Hengl T, Heuvelink GBM, Kempen B, Leenaars JGB, Walsh MG, Shepherd KD, Sila A, MacMillan RA, Mendes de Jesus J, Tamene L, et al. 2015. Mapping Soil Properties of Africa at $250 \mathrm{~m}$ Resolution: Random Forests Significantly Improve Current Predictions. PLOS ONE 10 (6): e0125814 DOI: 10.1371/journal.pone.0125814

Hewlett JD, Hibbert AR. 1967. Factors affecting the response of small watersheds to precipitation in humid areas. Forest hydrology: 275-290 DOI: 10.1177/0309133309338118

Hook PB. 2003. Sediment Retention in Rangeland Riparian Buffers. Journal of Environment Quality 32 (3): 1130 DOI: $10.2134 /$ jeq2003.1130

Hooke J. 2003. Coarse sediment connectivity in river channel systems: a conceptual framework and methodology. Geomorphology 56 (1-2): 79-94 D0I: 10.1016/S0169-555X(03)00047-3

Hornberger GM, Bencala KE, McKnight DM. 1994. Hydrological controls on dissolved organic carbon during snowmelt in the Snake River near Montezuma, Colorado. Biogeochemistry 25 (3): 147-165 DOI: $10.1007 /$ BF00024390

Howard AD, Keetch ME, Vincent CL. 1970. Topological and Geometrical Properties of Braided Streams. Water Resources Research 6 (6): 1674-1688 DOI: 10.1029/WR006i006p01674

Huang C, Gascuel-Odoux C, Cros-Cayot S. 2002. Hillslope topographic and hydrologic effects on overland flow and erosion. CATENA 46 (2-3): 177-188 DOI: 10.1016/S03418162(01)00165-5

Hummon NP, Dereian P. 1989. Connectivity in a citation network: The development of DNA theory. Social Networks 11 (1): 39-63 DOI: 10.1016/0378-8733(89)90017-8

Hunink JE, Droogers P, Kauffman S, Mwaniki BM, Bouma J. 2012. Quantitative simulation tools to analyze up- and downstream interactions of soil and water conservation measures: Supporting policy making in the Green Water Credits program of Kenya. Journal of Environmental Management 111: 187-194 DOI: 10.1016/j.jenvman.2012.07.022

Hunt A, Ewing R, Ghanbarian B. 2014. Percolation Theory for Flow in Porous Media. Springer International Publishing: Cham. DOI: 10.1007/978-3-319-03771-4

Irish RR. 2000. Landsat 7 automatic cloud cover assessment. In AeroSense 2000International Society for Optics and Photonics; 348-355. DOI: 10.1117/12.410358

Iserloh T, Ries JB, Arnáez J, Boix-Fayos C, Butzen V, Cerdà A, Echeverría MT, Fernández-Gálvez J, Fister W, Geißler C, et al. 2013. European small portable rainfall simulators: A comparison of rainfall characteristics. CATENA 110: 100-112 DOI: 10.1016/j.catena.2013.05.013

Jaeger KL, Olden JD. 2012. Electrical resistance sensor arrays as a means to quantify longitudinal connectivity of rivers. River Research and Applications 28 (10): 1843-1852 DOI: 10.1002/rra.1554

James AL, Roulet NT. 2007. Investigating hydrologic connectivity and its association with threshold change in runoff response in a temperate forested watershed. Hydrological Processes 21 (25): 3391-3408 D0I: 10.1002/hyp.6554 
Jasiewicz J, Stepinski TF. 2013. Geomorphons - a pattern recognition approach to classification and mapping of landforms. Geomorphology 182: 147-156 DOI: 10.1016/j.geomorph.2012.11.005

Javernick L, Brasington J, Caruso B. 2014. Modeling the topography of shallow braided rivers using Structure-from-Motion photogrammetry. Geomorphology 213: 166-182 DOI: 10.1016/j.geomorph.2014.01.006

Jones N, de Graaff J, Duarte F, Rodrigo I, Poortinga A. 2014. Farming systems in two less favoured areas in Portugal: their development from 1989 to 2009 and the implications for sustainable land management. Land Degradation \& Development 25 (1): 29-44 DOI: 10.1002/ldr.2257

Jordán F, Scheuring I. 2004. Network ecology: topological constraints on ecosystem dynamics. Physics of Life Reviews 1 (3): 139-172 DOI: 10.1016/j.plrev.2004.08.001

Kaiser A, Neugirg F, Haas F, Schmidt J, Becht M, Schindewolf M. 2015. Determination of hydrological roughness by means of close range remote sensing. SOIL 1 (2): 613-620 DOI: 10.5194/soil$1-613-2015$

Kalantari Z, Lyon SW, Jansson P-E, Stolte J, French HK, Folkeson L, Sassner M. 2015. Modeller subjectivity and calibration impacts on hydrological model applications: An event-based comparison for a road-adjacent catchment in south-east Norway. Science of The Total Environment 502: 315-329 DOI: 10.1016/j.scitotenv.2014.09.030

Kang Y, Wang Q-G, Liu H-J. 2005. Winter wheat canopy interception and its influence factors under sprinkler irrigation. Agricultural Water Management $\mathbf{7 4}$ (3): 189-199 DOI: 10.1016/j.agwat.2004.11.004

Kaspar TC, Radke JK, Laflen JM. 2001a. Small grain cover crops and wheel traffic effects on infiltration, runoff, and erosion. Journal of Soil and Water Conservation 56 (2): 160-164 DOI: $10.1177 / 0309133309338118$

Kaspar TC, Radke JK, Laflen JM. 2001b. Small grain cover crops and wheel traffic effects on infiltration, runoff, and erosion. Journal of Soil and Water Conservation 56 (2): 160-164

Keesstra S, Maroulis J, Argaman E, Voogt A, Wittenberg L. 2014a. Effects of controlled fire on hydrology and erosion under simulated rainfall. Cuadernos de Investigación Geográfica 40 (2): 269-294

Keesstra S, Pereira P, Novara A, Brevik EC, Azorin-Molina C, Parras-Alcántara L, Jordán A, Cerdà A. 2016. Effects of soil management techniques on soil water erosion in apricot orchards. Science of The Total Environment 551-552: 357-366 DOI: 10.1016/j.scitotenv.2016.01.182

Keesstra SD. 2007. Impact of natural reforestation on floodplain sedimentation in the Dragonja basin, SW Slovenia. Earth Surface Processes and Landforms 32 (1): 49-65 DOI: 10.1002/esp.1360

Keesstra SD, Bruijnzeel LA, van Huissteden J. 2009a. Meso-scale catchment sediment budgets: combining field surveys and modeling in the Dragonja catchment, southwest Slovenia. Earth Surface Processes and Landforms 34 (11): 1547-1561 DOI: 10.1002/esp.1846

Keesstra SD, van Dam 0, Verstraeten G, van Huissteden J. 2009b. Changing sediment dynamics due to natural reforestation in the Dragonja catchment, SW Slovenia. CATENA 78 (1): 60-71 DOI: 10.1016/j.catena.2009.02.021

Keesstra SD, Kondrlova E, Czajka A, Seeger M, Maroulis J. 2012. Assessing riparian zone impacts on water and sediment movement: a new approach. Netherlands Journal of Geosciences 91 (12): 245-255 DOI: $10.1017 /$ S0016774600001633

Keesstra SD, Nunes JP, Saco PM, Parsons AJ, Poeppl RE, Pereira P, Novara A, Rodrigo Comino J, Masselink R, Cerdà A. In review. The way forward: can connectivity be used for better measuring and modelling schemes for water and sediment dynamics? Earth-Science Reviews

Keesstra SD, Temme AJAM, Schoorl JM, Visser SM. 2014b. Evaluating the hydrological component of the new catchment-scale sediment delivery model LAPSUS-D. Geomorphology 212: 97-107 DOI: 10.1016/j.geomorph.2013.04.021 
Keesstra SD, van Dam 0, Verstraeten G, van Huissteden J. 2009c. Changing sediment dynamics due to natural reforestation in the Dragonja catchment, SW Slovenia. CATENA 78 (1): 60-71 DOI: 10.1016/j.catena.2009.02.021

Kendall C, McDonnell JJ. 2012. Isotope tracers in catchment hydrology. Elsevier.

Khanbilvardi RM, Rogowski AS. 1984. Quantitative Evaluation of sediment delivery ratios. Journal of the American Water Resources Association 20 (6): 865-874 DOI: 10.1111/j.17521688.1984.tb04794.x

Kimoto A, Nearing MA, Shipitalo MJ, Polyakov V0. 2006. Multi-year tracking of sediment sources in a small agricultural watershed using rare earth elements. Earth Surface Processes and Landforms 31 (14): 1763-1774 DOI: 10.1002/esp.1355

Kininmonth S, Bergsten A, Bodin Ö. 2015. Closing the collaborative gap: Aligning social and ecological connectivity for better management of interconnected wetlands. AMBIO 44 (S1): 138-148 DOI: $10.1007 / \mathrm{s} 13280-014-0605-9$

Kirkby M. 1971. Hillslope process-response models based on the continuity equation. Institute of British Geographers Special Publication 3: 15-30

Kirkby M, Bracken L, Reaney S. 2002. The influence of land use, soils and topography on the delivery of hillslope runoff to channels in SE Spain. Earth Surface Processes and Landforms 27 (13): 1459-1473

Kirkby MJ. 2014. Do not only connect: a model of infiltration-excess overland flow based on simulation. Earth Surface Processes and Landforms 39 (7): 952-963 DOI: 10.1002/esp.3556

Knudby C, Carrera J. 2005. On the relationship between indicators of geostatistical, flow and transport connectivity. Advances in Water Resources 28 (4): 405-421 DOI: 10.1016/j.advwatres.2004.09.001

Kohler MA, Linsley RK. 1951. Predicting the runoff from storm rainfall. U.S. Weather Bureau, Washington Research paper no. 34

Kraus K, Pfeifer N. 1998. Determination of terrain models in wooded areas with airborne laser scanner data. ISPRS Journal of Photogrammetry and Remote Sensing 53 (4): 193-203 DOI: 10.1016/S0924-2716(98)00009-4

Lane SN, Brookes CJ, Kirkby MJ, Holden J. 2004. A network-index-based version of TOPMODEL for use with high-resolution digital topographic data. Hydrological Processes 18 (1): 191-201 DOI: 10.1002/hyp.5208

Lane SN, Reaney SM, Heathwaite AL. 2009. Representation of landscape hydrological connectivity using a topographically driven surface flow index. Water Resources Research 45 (8) DOI: 10.1029/2008WR007336

Le Bissonnais Y, Renaux B, Delouche H. 1995. Interactions between soil properties and moisture content in crust formation, runoff and interrill erosion from tilled loess soils. CATENA 25 (14): 33-46 DOI: 10.1016/0341-8162(94)00040-L

Lesschen JP, Schoorl JM, Cammeraat LH. 2009. Modelling runoff and erosion for a semi-arid catchment using a multi-scale approach based on hydrological connectivity. Geomorphology 109 (3-4): 174-183 DOI: 10.1016/j.geomorph.2009.02.030

Leuchter AF, Newton TF, Cook IA, Walter DO, Rosenberg-Thompson S, Lachenbruch PA. 1992. Changes in brain functional connectivity in alzheimer-type and multi-infarct dementia. Brain 115 (5): 1543-1561 DOI: 10.1093/brain/115.5.1543

Lexartza-Artza I, Wainwright J. 2009. Hydrological connectivity: Linking concepts with practical implications. Catena 79: 146-152 DOI: 10.1016/j.catena.2009.07.001

Lillesand T, Kiefer RW, Chipman J. 2014. Remote sensing and image interpretation. John Wiley \& Sons.

Lisle IG, Rose CW, Hogarth WL, Hairsine PB, Sander GC, Parlange J-Y. 1998. Stochastic sediment transport in soil erosion. Journal of Hydrology 204 (1-4): 217-230 DOI: 10.1016/S00221694(97)00123-6 
Loch RJ. 2000. Effects of vegetation cover on runoff and erosion under simulated rain and overland flow on a rehabilitated site on the Meandu Mine, Tarong, Queensland. Soil Research 38 (2): 299-312

López-Vicente M, Nadal-Romero E, Cammeraat ELH. 2016. Hydrological connectivity does change over 70 years of abandonment and afforestation in the spanish pyrenees. Land Degradation \& Development DOI: $10.1002 /$ ldr.2531

López-Vicente M, Navas A, Gaspar L, Machín J. 2013a. Advanced modelling of runoff and soil redistribution for agricultural systems: The SERT model. Agricultural Water Management 125: 1-12 DOI: $10.1016 /$ j.agwat.2013.04.002

López-Vicente M, Poesen J, Navas A, Gaspar L. 2013b. Predicting runoff and sediment connectivity and soil erosion by water for different land use scenarios in the Spanish Pre-Pyrenees. CATENA 102: 62-73 DOI: 10.1016/j.catena.2011.01.001

Lucieer A, Robinson SA, Turner D. 2011. Unmanned aerial vehicle (UAV) remote sensing for hyperspatial terrain mapping of Antarctic moss beds based on structure from motion (SfM) point clouds

Maner SB. 1958. Factors affecting sediment delivery rates in the red hills physiographic area. Transactions, American Geophysical Union 39 (4): 669 DOI: 10.1029/TR039i004p00669

Marchamalo M, Hooke JM, Sandercock PJ. 2015. Flow and Sediment Connectivity in Semi-Arid Landscapes in SE Spain: Patterns and Controls. Land Degradation \& Development DOI: 10.1002/ldr.2352

Marra WA, Kleinhans MG, Addink EA. 2014. Network concepts to describe channel importance and change in multichannel systems: test results for the Jamuna River, Bangladesh. Earth Surface Processes and Landforms 39 (6): 766-778 DOI: 10.1002/esp.3482

Masselink RJH, Heckmann T, Temme AJAM, Anders NS, Gooren HPA, Keesstra SD. 2017. A network theory approach for a better understanding of overland flow connectivity. Hydrological Processes 31 (1): 207-220 DOI: 10.1002/hyp.10993

Masselink RJH, Keesstra SD, Temme AJAM, Seeger M, Giménez R, Casalí J. 2016. Modelling Discharge and Sediment Yield at Catchment Scale Using Connectivity Components. Land Degradation \& Development 27 (4): 933-945 DOI: 10.1002/ldr.2512

Mayor AG, Bautista S, Small EE, Dixon M, J B. 2008. Measurement of the connectivity of runoff source areas as determined by vegetation pattern and topography: A tool for assessing potential water and soil losses in drylands. Water Resources Research 44 (W10423) DOI: 10.1029/2007WR006367

McMillan HK, Clark MP, Bowden WB, Duncan M, Woods RA. 2011. Hydrological field data from a modeller's perspective: Part 1. Diagnostic tests for model structure. Hydrological Processes 25 (4): 511-522 DOI: 10.1002/hyp.7841

McRae BH, Dickson BG, Keitt TH, Shah VB. 2008. Using circuit theory to model connectivity in ecology, evolution, and conservation. Ecology 89 (10): 2712-2724 DOI: 10.1890/07-1861.1

Meerkerk AL, van Wesemael B, Bellin N. 2009. Application of connectivity theory to model the impact of terrace failure on runoff in semi-arid catchments. Hydrological Processes 23 (19): 27922803 DOI: 10.1002/hyp.7376

Mekonnen M, Keesstra SD, Baartman JE, Ritsema CJ, Melesse AM. 2015a. Evaluating sediment storage dams: structural off-site sediment trapping measures in northwest Ethiopia. Cuadernos de Investigación Geográfica 41 (1): 7 DOI: 10.18172/cig.2643

Mekonnen M, Keesstra SD, Baartman JEM, Stroosnijder L, Maroulis J. 2016. Reducing Sediment Connectivity Through man-Made and Natural Sediment Sinks in the Minizr Catchment, Northwest Ethiopia: Sediment Trapping Approach Lack Integration. Land Degradation \& Development DOI: $10.1002 / \mathrm{ldr} .2629$ 
Mekonnen M, Keesstra SD, Stroosnijder L, Baartman JEM, Maroulis J. 2015b. Soil Conservation Through Sediment Trapping: A Review. Land Degradation \& Development 26 (6): 544-556 DOI: 10.1002/ldr.2308

Merritt WS, Letcher RA, Jakeman AJ. 2003. A review of erosion and sediment transport models. Environmental Modelling \& Software 18 (8-9): 761-799 DOI: 10.1016/S13648152(03)00078-1

Meyles E, Williams A, Ternan L, Dowd J. 2003. Runoff generation in relation to soil moisture patterns in a small Dartmoor catchment, Southwest England. Hydrological Processes 17 (2): 251-264 DOI: $10.1002 /$ hyp. 1122

Michaelides K, Wainwright J. 2002. Modelling the effects of hillslope-channel coupling on catchment hydrological response. Earth Surface Processes and Landforms 27 (13): 1441-1457 DOI: 10.1002 /esp. 440

Milan DJ, Heritage GL, Hetherington D. 2007. Application of a 3D laser scanner in the assessment of erosion and deposition volumes and channel change in a proglacial river. Earth Surface Processes and Landforms 32 (11): 1657-1674 DOI: 10.1002/esp.1592

Minor ES, Urban DL. 2007. Graph theory as a proxy for spatially explicit population models in conservation planning. Ecological Applications 17 (6): 1771-1782 DOI: 10.1890/06-1073.1

Moody JA, Martin RG. 2015. Measurements of the initiation of post-wildfire runoff during rainstorms using in situ overland flow detectors. Earth Surface Processes and Landforms 40 (8): 10431056 DOI: $10.1002 /$ esp.3704

Moreno-de las Heras M, Saco PM, Willgoose GR, Tongway DJ. 2012. Variations in hydrological connectivity of Australian semiarid landscapes indicate abrupt changes in rainfall-use efficiency of vegetation. Journal of Geophysical Research 117 (G3) DOI: 10.1029/2011JG001839

Morgan RPC. 2009. Soil erosion and conservation. John Wiley \& Sons.

Moriasi DN, Arnold JG, Van Liew MW, Bingner RL, Harmel RD, Veith TL. 2007. Model evaluation guidelines for systematic quantification of accuracy in watershed simulations. Trans. Asabe 50 (3): 885-900

Mueller EN, Wainwright J, Parsons AJ. 2007. Impact of connectivity on the modeling of overland flow within semiarid shrubland environments. Water Resources Research 43 (9) DOI: 10.1029/2006WR005006

Nadal-Romero E, Revuelto J, Errea P, López-Moreno J. 2015. The application of terrestrial laser scanner and SfM photogrammetry in measuring erosion and deposition processes in two opposite slopes in a humid badlands area (central Spanish Pyrenees). SOIL 1 (2): 561-573

Nash JE, Sutcliffe JV. 1970. River flow forecasting through conceptual models part I - A discussion of principles. Journal of Hydrology 10 (3): 282-290 DOI: 10.1016/0022-1694(70)90255-6

Navarra. 2001. Estudio Agroclimaticó de Navarra (CD). Gobierno de Navarra, Departamento de Agricultura, Ganadería y Alimentación. Servicio de Estructuras Agrarias. Pamplona, España

Nearing MA. 1998. Why soil erosion models over-predict small soil losses and under-predict large soil losses. CATENA 32 (1): 15-22 DOI: 10.1016/S0341-8162(97)00052-0

Nearing MA. 2000. Evaluating soil erosion models using measured plot data: accounting for variability in the data. Earth Surface Processes and Landforms 25 (9): 1035-1043 DOI: 10.1002/10969837(200008)25:9<1035::AID-ESP121>3.0.CO;2-B

Newman M. 2010. Networks: an introduction. Oxford University Press.

Nord G, Esteves M. 2005. PSEM_2D: A physically based model of erosion processes at the plot scale. Water Resources Research 41 (8) DOI: 10.1029/2004WR003690

Nunes JP, Seixas J, Keizer JJ, Ferreira AJD. 2009. Sensitivity of runoff and soil erosion to climate change in two Mediterranean watersheds. Part I: model parameterization and evaluation. Hydrological Processes 23 (8): 1202-1211 DOI: 10.1002/hyp.7247 
Ocampo CJ, Sivapalan M, Oldham C. 2006. Hydrological connectivity of upland-riparian zones in agricultural catchments: Implications for runoff generation and nitrate transport. Journal of Hydrology 331 (3-4): 643-658 DOI: 10.1016/j.jhydrol.2006.06.010

Okin GS, Heras MM las, Saco PM, Throop HL, Vivoni ER, Parsons AJ, Wainwright J, Peters DP. 2015. Connectivity in dryland landscapes: shifting concepts of spatial interactions. Frontiers in Ecology and the Environment 13 (1): 20-27 DOI: 10.1890/140163

Ola A, Dodd IC, Quinton JN. 2015. Can we manipulate root system architecture to control soil erosion? SOIL 1 (2): 603-612 DOI: 10.5194/soil-1-603-2015

Park SW, Mitchell JK, Bubenzer GD. 1982. splash Erosion Modeling: Physical Analyses. Transactions of the ASAE 25 (2): 0357-0361 DOI: 10.13031/2013.33535

Parsons AJ, Bracken L, Poeppl RE, Wainwright J, Keesstra SD. 2015. Introduction to special issue on connectivity in water and sediment dynamics. Earth Surface Processes and Landforms 40 (9): 1275-1277 DOI: $10.1002 /$ esp.3714

Parsons AJ, Brazier RE, Wainwright J, Powell DM. 2006. Scale relationships in hillslope runoff and erosion. Earth Surface Processes and Landforms 31 (11): 1384-1393 DOI: 10.1002/esp.1345

Peñuela A, Darboux F, Javaux M, Bielders CL. 2016. Evolution of overland flow connectivity in bare agricultural plots. Earth Surface Processes and Landforms DOI: 10.1002/esp.3938

Peñuela A, Javaux M, Bielders CL. 2012. Scale effect on overland flow connectivity at the plot scale. Hydrology and Earth System Sciences Discussions 9 (6): 7877-7918 DOI: 10.5194/hessd-97877-2012

Peñuela A, Javaux M, Bielders CL. 2015. How do slope and surface roughness affect plot-scale overland flow connectivity? Journal of Hydrology 528: 192-205 DOI: 10.1016/j.jhydrol.2015.06.031

Phillips JD, Schwanghart W, Heckmann T. 2015. Graph theory in the geosciences. Earth-Science Reviews 143: 147-160 DOI: 10.1016/j.earscirev.2015.02.002

Phillips RW, Spence C, Pomeroy JW. 2011. Connectivity and runoff dynamics in heterogeneous basins. Hydrological Processes: 3061-3075 DOI: 10.1002/hyp.8123

Poeppl RE, Keesstra SD, Maroulis J. 2016. A conceptual connectivity framework for understanding geomorphic change in human-impacted fluvial systems. Geomorphology DOI: 10.1016/j.geomorph.2016.07.033

Poeppl RE, Keiler M, Von Elverfeldt K, Zweimueller I, Glade T. 2012. The influence of riparian vegetation cover on diffuse lateral sediment connectivity and biogeomorphic processes in a mediumsized agricultural catchment, Austria. Geografiska Annaler: Series A, Physical Geography 94 (4): 511-529 DOI: 10.1111/j.1468-0459.2012.00476.x

Poesen JWA, Hooke JM. 1997. Erosion, flooding and channel management in Mediterranean environments of southern Europe. Progress in Physical Geography 21 (2): 157-199 DOI: $10.1177 / 030913339702100201$

Polyakov VO, Nearing MA, Shipitalo MJ. 2004. Tracking sediment redistribution in a small watershed: implications for agro-landscape evolution. Earth Surface Processes and Landforms 29 (10): 1275-1291 DOI: 10.1002/esp.1094

Poole GC. 2002. Fluvial landscape ecology: addressing uniqueness within the river discontinuum. Freshwater Biology 47 (4): 641-660 DOI: 10.1046/j.1365-2427.2002.00922.x

Pringle C. 2003. The need for a more predictive understanding of hydrologic connectivity. Aquatic Conservation: Marine and Freshwater Ecosystems 13 (6): 467-471 DOI: 10.1002/aqc.603

Prosdocimi M, Cerdà A, Tarolli P. 2016a. Soil water erosion on Mediterranean vineyards: A review. CATENA 141: 1-21

Prosdocimi M, Jordán A, Tarolli P, Keesstra S, Novara A, Cerdà A. 2016b. The immediate effectiveness of barley straw mulch in reducing soil erodibility and surface runoff generation in Mediterranean vineyards. Science of The Total Environment 547: 323-330 
Riedel T, Brunner U. 1994. Traffic control using graph theory. Control Engineering Practice 2 (3): 397404 DOI: $10.1016 / 0967-0661(94) 90776-5$

Rodrigo Comino J, Iserloh T, Morvan X, Malam Issa 0, Naisse C, Keesstra S, Cerdà A, Prosdocimi M, Arnáez J, Lasanta T, et al. 2016. Soil Erosion Processes in European Vineyards: A Qualitative Comparison of Rainfall Simulation Measurements in Germany, Spain and France. Hydrology 3 (1): 6 DOI: 10.3390/hydrology3010006

Roehl JW. 1962. Sediment source areas, delivery ratios and influencing morphological factors. International Association of Scientific Hydrology 59: 202-213

Romero-Diaz A, Belmonte-Serrato F, Ruiz-Sinoga JD. 2010. The geomorphic impact of afforestations on soil erosion in Southeast Spain. Land Degradation \& Development 21 (2): 188-195 DOI: $10.1002 / \mathrm{ldr} .946$

de Roo APJ, Wesseling CG, Ritsema CJ. 1996. LISEM: a single-event physically based hydrological and soil erosion model for drainage basins. i: theory, input and output. Hydrological Processes 10 (8): $\quad 1107-1117 \quad$ DOI: $\quad 10.1002 /(S I C I) 1099-1085(199608) 10: 8<1107:: A I D-$ HYP415>3.0.CO;2-4

Sadeghi SHR, Mizuyama T, Miyata S, Gomi T, Kosugi K, Fukushima T, Mizugaki S, Onda Y. 2008. Determinant factors of sediment graphs and rating loops in a reforested watershed. Journal of Hydrology 356 (3-4): 271-282 DOI: 10.1016/j.jhydrol.2008.04.005

Sayama T, McDonnell JJ. 2009. A new time-space accounting scheme to predict stream water residence time and hydrograph source components at the watershed scale. Water Resources Research 45 (7) DOI: 10.1029/2008WR007549

Scherrer S, Naef F, Faeh A0, Cordery I. 2007. Formation of runoff at the hillslope scale during intense precipitation. Hydrology and Earth System Sciences 11 (2): 907-922 DOI: 10.5194/hess-11907-2007

Schmitz C, van Meijl H, Kyle P, Nelson GC, Fujimori S, Gurgel A, Havlik P, Heyhoe E, d'Croz DM, Popp A, et al. 2014. Land-use change trajectories up to 2050: insights from a global agro-economic model comparison. Agricultural Economics 45 (1): 69-84 DOI: 10.1111/agec.12090

Schoorl JM, Sonneveld MPW, Veldkamp A. 2000. Three-dimensional landscape process modelling: the effect of DEM resolution. Earth Surface Processes and Landforms 25 (9): 1025-1034 DOI: 10.1002/1096-9837(200008)25:9<1025::AID-ESP116>3.0.CO;2-Z

Schoorl JM, Veldkamp A, Bouma J. 2002. Modeling Water and Soil Redistribution in a Dynamic Landscape Context. Soil Science Society of America Journal 66 (5): 1610 DOI: $10.2136 /$ sssaj2002.1610

Schwanghart W, Heckmann T. 2012. Fuzzy delineation of drainage basins through probabilistic interpretation of diverging flow algorithms. Environmental Modelling \& Software 33: 106113 DOI: $10.1016 /$ j.envsoft.2012.01.016

Schwanghart W, Kuhn NJ. 2010. TopoToolbox: A set of Matlab functions for topographic analysis. Environmental Modelling \& Software 25 (6): 770-781 DOI: 10.1016/j.envsoft.2009.12.002

Seeger M, Errea M-P, Beguería S, Arnáez J, Martí C, García-Ruiz J. 2004. Catchment soil moisture and rainfall characteristics as determinant factors for discharge/suspended sediment hysteretic loops in a small headwater catchment in the Spanish pyrenees. Journal of Hydrology 288 (34): 299-311 DOI: 10.1016/j.jhydrol.2003.10.012

Selman P, Doar N. 1992. An investigation of the potential for landscape ecology to act as a basis for rural land use plans. Journal of Environmental Management 35 (4): 281-299 DOI: 10.1016/S0301-4797(11)80011-3

Serpa D, Nunes JP, Santos J, Sampaio E, Jacinto R, Veiga S, Lima JC, Moreira M, Corte-Real J, Keizer JJ, et al. 2015. Impacts of climate and land use changes on the hydrological and erosion processes of two contrasting Mediterranean catchments. Science of The Total Environment 538: 64-77 DOI: $10.1016 /$ j.scitotenv.2015.08.033 
Sherriff SC, Rowan JS, Fenton O, Jordan P, Melland AR, Mellander P-E, hUallacháin DÓ. 2016. Storm Event Suspended Sediment-Discharge Hysteresis and Controls in Agricultural Watersheds: Implications for Watershed Scale Sediment Management. Environmental Science \& Technology 50 (4): 1769-1778 DOI: 10.1021/acs.est.5b04573

Shreve RL. 1974. Variation of mainstream length with basin area in river networks. Water Resources Research 10 (6): 1167-1177 DOI: 10.1029/WR010i006p01167

Slaymaker 0. 2003. The sediment budget as conceptual framework and management tool. In The Interactions between Sediments and WaterSpringer; 71-82.

Smetanová A, Paton EN, Maynard C, Tindale S, Fernández-Getino AP, Marqués Pérez MJ, Bracken LJ, Le Bissonnais Y, Keesstra SD. In review. Stakeholders' Perception On The Relevance Of Water And Sediment Connectivity In Water And Land Management. Land Degradation \& Development

Smith MW, Vericat D. 2015. From experimental plots to experimental landscapes: topography, erosion and deposition in sub-humid badlands from Structure-from-Motion photogrammetry: MultiScale Validation of Structure from Motion in a Badland Setting. Earth Surface Processes and Landforms 40 (12): 1656-1671 DOI: 10.1002/esp.3747

Spence C. 2010. A Paradigm Shift in Hydrology: Storage Thresholds Across Scales Influence Catchment Runoff Generation: Paradigm shift in hydrology. Geography Compass 4 (7): 819-833 DOI: 10.1111/j.1749-8198.2010.00341.x

Srinivasan MS, Gburek WJ, Hamlett JM. 2002. Dynamics of stormflow generation-A hillslope-scale field study in east-central Pennsylvania, USA. Hydrological Processes 16 (3): 649-665 DOI: 10.1002/hyp.311

Srinivasan MS, Wittman MA, Hamlett JM, Gburek WJ. 2001. Surface and subsurface sensors to record variable runoff generation areas. Transactions of the American Society of Agricultural Engineers 43 (3): 651-660

Stieglitz M. 2003. An approach to understanding hydrologic connectivity on the hillslope and the implications for nutrient transport. Global Biogeochemical Cycles 17 (4) DOI: 10.1029/2003GB002041

Stieglitz M, Shaman J, McNamara J, Engel V, Shanley J, Kling GW. 2003. An approach to understanding hydrologic connectivity on the hillslope and the implications for nutrient transport. Global Biogeochemical Cycles 17 (4) DOI: 10.1029/2003GB002041

Stoof CR, Moore D, Ritsema CJ, Dekker LW. 2011. Natural and Fire-Induced Soil Water Repellency in a Portuguese Shrubland. Soil Science Society of America Journal 75 (6): 2283 DOI: 10.2136/sssaj2011.0046

Strahler AN. 1957. Quantitative analysis of watershed geomorphology. Transactions, American Geophysical Union 38 (6): 913 DOI: 10.1029/TR038i006p00913

Stubbs DF. 1978. Connectivity and the Brain. Kybernetes 7 (2): 93-98 DOI: 10.1108/eb005473

Tarboton DG. 1997. A new method for the determination of flow directions and upslope areas in grid digital elevation models. Water Resources Research 33 (2): 309-319 DOI: 10.1029/96WR03137

Tavares Wahren F, Julich S, Nunes JP, Gonzalez-Pelayo O, Hawtree D, Feger K-H, Keizer JJ. 2016. Combining digital soil mapping and hydrological modeling in a data scarce watershed in north-central Portugal. Geoderma 264: 350-362 DOI: 10.1016/j.geoderma.2015.08.023

Temme A, Schoorl J, Claessens L, Veldkamp A, Shroder F. 2013. Quantitative Modeling of Landscape Evolution, Treatise on Geomorphology. In Quantitative Modeling of GeomorphologyAcademic Press; $180-200$.

Temme AJAM, Veldkamp A. 2009. Multi-process Late Quaternary landscape evolution modelling reveals lags in climate response over small spatial scales. Earth Surface Processes and Landforms 34 (4): 573-589 DOI: 10.1002/esp.1758 
Tetzlaff D, Soulsby C, Bacon PJ, Youngson AF, Gibbins C, Malcolm IA. 2007. Connectivity between landscapes and riverscapes-a unifying theme in integrating hydrology and ecology in catchment science? Hydrological Processes 21 (10): 1385-1389 D0I: 10.1002/hyp.6701

Thompson CJ, Fryirs K, Croke J. 2016. The Disconnected Sediment Conveyor Belt: Patterns of Longitudinal and Lateral Erosion and Deposition During a Catastrophic Flood in the Lockyer Valley, South East Queensland, Australia: The Disconnected Sediment Conveyor Belt. River Research and Applications 32 (4): 540-551 DOI: 10.1002/rra.2897

Treml EA, Halpin PN, Urban DL, Pratson LF. 2008. Modeling population connectivity by ocean currents, a graph-theoretic approach for marine conservation. Landscape Ecology 23 (S1): 19-36 DOI: 10.1007/s10980-007-9138-y

Trevisani S, Cavalli M. 2016. Topography-based flow-directional roughness: potential and challenges. Earth Surface Dynamics 4 (2): 343-358 DOI: 10.5194/esurf-4-343-2016

Tucker GE, Hancock GR. 2010. Modelling landscape evolution. Earth Surface Processes and Landforms 35 (1): 28-50 DOI: 10.1002/esp.1952

Turnbull L, Hùtt M., Ioannides A, Kininmonth S, Poeppl R, Tockner C, Bracken LJ, Keesstra SD, Liu L, Masselink RJH, et al. In prep. Connectivity and complex systems: learning from a multidisciplinary perspective. Journal of the Royal Society Interface

Turnbull L, Wainwright J, Brazier RE. 2008. A conceptual framework for understanding semi-arid land degradation: ecohydrological interactions across multiple-space and time scales. Ecohydrology 1: 23-34 DOI: 10.1002/eco.4

United States Geological Survey U. 2015. SLC-off Products: Background. Landsat 7 SLC-OFF Available at: http://landsat.usgs.gov/products_slcoffbackground.php [Accessed 3 February 2016]

Urban D, Keitt T. 2001. Landscape connectivity: a graph-theoretic perspective. Ecology 82 (5): 12051218 DOI: 10.1890/0012-9658(2001)082[1205:LCAGTP]2.0.CO;2

Vanacker V, Molina A, Govers G, Poesen J, Dercon G, Deckers S. 2005. River channel response to shortterm human-induced change in landscape connectivity in Andean ecosystems. Geomorphology 72 (1-4): 340-353 DOI: 10.1016/j.geomorph.2005.05.013

de Vente J, Poesen J. 2005. Predicting soil erosion and sediment yield at the basin scale: Scale issues and semi-quantitative models. Earth-Science Reviews 71 (1-2): 95-125 DOI: 10.1016/j.earscirev.2005.02.002

de Vente J, Poesen J, Arabkhedri M, Verstraeten G. 2007. The sediment delivery problem revisited. Progress in Physical Geography 31 (2): 155-178 DOI: 10.1177/0309133307076485

de Vente J, Poesen J, Bazzoffi P, Rompaey AV, Verstraeten G. 2006. Predicting catchment sediment yield in Mediterranean environments: the importance of sediment sources and connectivity in Italian drainage basins. Earth Surface Processes and Landforms 31 (8): 1017-1034 DOI: 10.1002/esp.1305

Verstraeten G, Poesen J, Gillijns K, Govers G. 2006. The use of riparian vegetated filter strips to reduce river sediment loads: an overestimated control measure? Hydrological Processes 20 (20): 4259-4267 DOI: 10.1002/hyp.6155

Vidon PGF, Hill AR. 2004. Landscape controls on nitrate removal in stream riparian zones. Water Resources Research 40 (3) DOI: 10.1029/2003WR002473

Vigiak O, Borselli L, Newham LTH, McInnes J, Roberts AM. 2012. Comparison of conceptual landscape metrics to define hillslope-scale sediment delivery ratio. Geomorphology 138 (1): 74-88 DOI: 10.1016/j.geomorph.2011.08.026

Wainwright J, Parsons AJ, Abrahams AD. 2000. Plot-scale studies of vegetation, overland flow and erosion interactions: case studies from Arizona and New Mexico. Hydrological Processes 14 (16-17): 2921-2943 DOI: 10.1002/1099-1085(200011/12)14:16/17<2921::AIDHYP127>3.0.CO;2-7 
Wainwright J, Parsons AJ, Müller EN, Brazier RE, Powell DM, Fenti B. 2008. A transport-distance approach to scaling erosion rates: 1. Background and model development. Earth Surface Processes and Landforms 33 (5): 813-826 DOI: 10.1002/esp.1624

Wainwright J, Turnbull L, Ibrahim TG, Lexartza-Artza I, Thornton SF, Brazier RE. 2011. Linking environmental régimes, space and time: Interpretations of structural and functional connectivity. Gemorphology 126: 387-404 DOI: 10.1016/j.geomorph.2010.07.027

Walling DE. 1983. The sediment delivery problem. Journal of Hydrology 65 (1-3): 209-237 DOI: 10.1016/0022-1694(83)90217-2

Walling DE, Collins AL. 2008. The catchment sediment budget as a management tool. Environmental Science \& Policy 11 (2): 136-143 DOI: 10.1016/j.envsci.2007.10.004

Ward JV. 1989. The Four-Dimensional Nature of Lotic Ecosystems. Journal of the North American Benthological Society 8 (1): 2-8 DOI: 10.2307/1467397

Werner C. 1994. Explorations into the formal structure of drainage basins. Earth Surface Processes and Landforms 19 (8): 747-762 DOI: 10.1002/esp.3290190807

Western AW, Blöschl G, Grayson RB. 2001. Toward capturing hydrologically significant connectivity in spatial patterns. Water Resources Research 37 (1): 83-97

Westoby MJ, Brasington J, Glasser NF, Hambrey MJ, Reynolds JM. 2012. 'Structure-from-Motion' photogrammetry: A low-cost, effective tool for geoscience applications. Geomorphology 179: 300-314 DOI: 10.1016/j.geomorph.2012.08.021

Wickama J, Masselink R, Sterk G. 2015. The effectiveness of soil conservation measures at a landscape scale in the West Usambara highlands, Tanzania. Geoderma 241-242: 168-179 DOI: 10.1016/j.geoderma.2014.11.020

van Wijk BCM, Stam CJ, Daffertshofer A. 2010. Comparing Brain Networks of Different Size and Connectivity Density Using Graph Theory (O Sporns, ed.). PLoS ONE 5 (10): e13701 DOI: 10.1371/journal.pone.0013701

Wischmeier W., Smith DD. 1978. Predicting rainfall erosion losses-A guide to conservation planning.

Yong B, Ren L-L, Hong Y, Gourley JJ, Chen X, Zhang Y-J, Yang X-L, Zhang Z-X, Wang W-G. 2012. A novel multiple flow direction algorithm for computing the topographic wetness index. Hydrology Research 43 (1-2): 135 DOI: 10.2166/nh.2011.115

Yuan Y, Jiang Y, Taguas EV, Mbonimpa EG, Hu W. 2015. Sediment loss and its cause in Puerto Rico watersheds. SOIL 1 (2): 595-602 DOI: 10.5194/soil-1-595-2015

Zabaleta A, Martínez M, Uriarte JA, Antigüedad I. 2007. Factors controlling suspended sediment yield during runoff events in small headwater catchments of the Basque Country. CATENA 71 (1): 179-190 DOI: 10.1016/j.catena.2006.06.007

Zhang XC, Friedrich JM, Nearing MA, Norton LD. 2001. Potential use of Rare Earth Oxides as Tracers for Soil Erosion and Aggregation Studies. Soil Science Society of America Journal 65 (5): 1508 DOI: $10.2136 /$ sssaj2001.6551508x

Ziegler AD, Giambelluca TW, Tran LT, Vana TT, Nullet MA, Fox J, Vien TD, Pinthong J, Maxwell J., Evett S. 2004. Hydrological consequences of landscape fragmentation in mountainous northern Vietnam: evidence of accelerated overland flow generation. Journal of Hydrology 287 (1-4): 124-146 DOI: 10.1016/j.jhydrol.2003.09.027

Zilles K, Schleicher A, Palomero-Gallagher N, Amunts K. 2002. Quantitative Analysis of Cyto- and Receptor Architecture of the Human Brain. In Brain Mapping: The MethodsElsevier; 573-602. 


\section{Summary}

Land degradation is a large problem worldwide, especially in agricultural areas. Between 1-6 billion ha of land worldwide is affected by land degradation. With an increasing world population, more food production is needed and, therefore, more land is converted into agricultural areas. This conversion of land to agricultural areas, in turn, leads to more land degradation. Some common forms of land degradation are desertification, salinization and soil erosion by water. The negative effects of soil erosion have been recognized for a long time. Since the early $20^{\text {th }}$ century, researchers have tried to quantify soil displaced due to water, and to measure and model the efficiency of management strategies.

The implications of problems with upscaling, wrong process representation and equifinality include the difficulty to properly predict sediment sources, pathways and sinks within catchments. These problems then can translate into the implementation of sub-optimal management strategies. To deal with these non-linear processes and the lack of proper representation of water and sediment sources, pathways and sinks, the concept of connectivity was developed. Currently, many definitions of connectivity have been proposed, although the definition most used is that of hydrological connectivity by Pringle (2003): 'Hydrologic connectivity is the water-mediated transport of matter, energy and organisms within or between elements of the hydrologic cycle'.

A unified theory on what constitutes connectivity and how connectivity should be measured or inferred remains one of the biggest challenges within catchment science. In addition, it is unclear whether connectivity should be an output or an input of a model and if an input, whether this should be added explicitly or implicitly. The main objective of this thesis was, therefore, to assess and quantify hydrological and sediment connectivity in a meaningful way, which can further our understanding of hydrological and sediment transport processes and catchment system dynamics.

The study was carried out in three catchments in Navarre, northern Spain. Two catchments, 'Latxaga' and 'La Tejeria', are agricultural catchments with sizes of 2.07 $\mathrm{km}^{2}$ and $1.69 \mathrm{~km}^{2}$, respectively. The 'Oskotz Forestal' catchment is a (semi-)natural catchment, with a size of $5.05 \mathrm{~km}^{2}$. Land cover in the agricultural catchments is mainly winter wheat and barley, while in the Oskotz catchment it is grassland and forest. Latxaga and La Tejeria are mainly underlain by marls and within La Tejeria some sandstone is also present. The geology in Oskotz is characterised by an alternation of marls and sandy limestone.

In chapter 2, I used networks (graph theory) to characterise and quantify overland flow connectivity dynamics on hillslopes in a humid sub-Mediterranean environment by using a combination of high-resolution digital-terrain models, overland flow sensors and a network approach. Results showed that there are significant differences between overland flow connectivity on agricultural areas and semi-natural shrubs areas. Significant positive correlations between connectivity and precipitation characteristics were found. Significant negative correlations between 
connectivity and soil moisture were found, most likely due to soil water repellency and/or soil surface crusting. The combination of structural networks and dynamic networks for determining potential connectivity and actual connectivity proved a powerful tool for analysing overland flow connectivity.

In chapter 3, I determined the functioning of hillslope-channel connectivity and the continuation of transport of these sediments in the channel. To determine this functioning, I obtained data on sediment transport from the hillslopes to the channels while simultaneously looking at factors that influence sediment export out of the catchment. For measuring hillslope-channel sediment connectivity, Rare-Earth Oxide (REO) tracers were applied to a hillslope in the Latxaga catchment preceding the winter of 2014-2015. The results showed that during the winter there have been no sediments transported from the hillslope into the channel. Analysis of precipitation data showed that although total precipitation quantities did not differ much from the mean, the precipitation intensities were low. Using a Random Forest (RF) machine learning method, I showed that hillslope-channel connectivity in Latxaga is dominated by sediment mobilisation during large (high intensity) precipitation events. Sediments are for a large part exported during those events. Large events also leave behind large amounts of sediments in and near the channel, which is gradually removed by small events.

In chapter 4 I demonstrated that existing data can be used to assess governing factors of connectivity, and how these factors change over time. Data from three catchments in Navarre, Northern Spain, were used to assess factors that influence hydrologic and sediment connectivity. These factors were used as components in a spatially-lumped linear model for discharge and suspended-sediment yield. Three components of connectivity were distinguished: topographical, biological and soil. Changes in the topographical component for the studied periods were considered relatively small, and, therefore, kept constant. Changes in the biological component were determined using the Normalised Difference Vegetation Index. Changes in the soil component were assessed using an Antecedent Precipitation Index. Nash-Sutcliffe model efficiency coefficients were between 0.49 through 0.62 for the discharge models and between 0.23 through 0.3 for the sediment-yield models. I recommended applying the model at smaller spatial scales than catchment scale to minimize the lumping of spatial variability in the components.

In chapter 5 , the objective was to better understand the implications of model calibration at different spatial scales on the simulation of hydrology and sediment dynamics of an agricultural catchment. I applied the LAPSUS-D model to the Latxaga catchment. The model was calibrated and validated (4 years: 2011-2015) using three datasets at varying spatial scales: hillslope, catchment and the combined dataset (combined-calibrated model). The hillslope-calibrated model showed mainly infiltration-excess overland flow, the catchment-calibrated mainly saturation-excess overland flow at the footslopes and the combined-calibrated model showed saturationexcess overland flow from the midslopes to the footslopes. For hydrology, the 
combined-calibrated model simulated the large discharge peaks best, while at the hillslope scale, the hillslope-calibrated model performed best. The hillslope-calibrated model produced the highest model efficiencies for sediments, for calibration (0.618) and validation (0.269). The hillslope-calibrated model was the only model that showed observed gully erosion on a high-resolution DEM and displayed channel sediment dynamics. However, absolute quantities of erosion and deposition within the catchment were too high. The results show that modellers need to be aware of problems associated with automatic calibration, over-calibration and not incorporating measured data at multiple spatial scales. We advocate incorporating runoff and sediment tracing data at multiple scales whenever this is possible and to, furthermore, carry out specific measuring campaigns towards this end, ultimately to get a more comprehensive view on hydrological and sediment connectivity within a catchment.

The combination of chapters in this thesis showed that the connectivity concept is useful for a wide range of studies, from hillslope scale to catchment scale. Using the concept, I was able to determine sediment dynamics for a humid-Mediterranean catchment and show that this behaviour is different than previously thought.

Depending of the aim of the study, various concepts of connectivity are useful. Different geologic and climatic settings cause large differences in catchment (sediment) dynamics. It might, therefore, not be necessary, or even possible, to strive for a single, unifying conceptual framework for connectivity. Instead, a collection of frameworks for different settings should be developed. These frameworks should, however, always aim at helping to understand which measurements need to be taken and which type of models and indices should be used for that particular setting.

It is my honest opinion that connectivity is definitely a useful concept to advance our knowledge on water and sediment transport processes further. However, careful consideration is also required as this particular concept will not necessary provide the ultimate explanation and insights in dynamic behaviour within watersheds around the world. The gap between the different spatial and temporal scales is too complex to be bridged with a single concept like connectivity. However, the many studies about connectivity that will be published in the near future will be able to advance knowledge on water and sediment transport processes. 


\section{Samenvatting}

Landdegradatie is een groot wereldwijd probleem, vooral in landbouwgebieden. Een gebied ter grootte van 1-6 miljard ha wordt wereldwijd aangetast door landdegradatie. Een groeiende wereldbevolking zorgt voor een hogere vraag naar voedsel, wat ervoor zorgt dat meer land wordt omgezet naar landbouwgrond, wat weer leidt tot meer landdegradatie. De meest voorkomende vormen van landdegradatie zijn verwoestijning, verzilting en bodemerosie door water. De negatieve effecten van erosie worden al lang herkend. Al vanaf het begin van de $20^{\text {ste }}$ eeuw hebben onderzoekers getracht te kwantificeren hoeveel bodemerosie plaatsvindt en om de effectiviteit van beheerstrategieën te meten en te modelleren.

Het blijft moeilijk om met voldoende nauwkeurigheid sedimentbronnen, paden en depositiegebieden te bepalen, o.a. door problemen met opschalen, verkeerde procesweergave en equifinaliteit. Deze problemen vertalen zich naar implementatie van sub-optimale beheerstrategieën. Om beter met deze problemen om te gaan is het concept 'connectivity' (Engels: mate van verbinding) bedacht. Er zijn tot op heden vele definities voor connectivity voorgesteld, maar de definitie die het meest gebruikt wordt is de definitie van hydrologische connectivity van Pringle (2003): "Hydrologische connectivity is transport door water van deeltjes, energie en organismes, in of tussen verschillende elementen in de hydrologische cyclus".

Een compleet overzicht over wat connectivity precies is en hoe het gemeten of afgeleid zou moeten worden, blijft echter ontbreken binnen het wetenschapsgebied van stroomgebiedanalyse. Daarnaast blijft het onduidelijk of connectivity een uitvoer of een invoer van een model zou moeten zijn en in het geval van een invoer, of het expliciet of impliciet moet worden toegevoegd aan het model. Het hoofddoel van dit proefschrift was daarom om hydrologische en sediment connectivity op een betekenisvolle manier te bestuderen en kwantificeren, waarmee onze kennis over hydrologische en sediment transportprocessen en stroomgebieddynamiek verbeterd wordt.

Dit onderzoek is uitgevoerd in drie stroomgebieden in Navarra, Noord Spanje. Twee stroomgebieden, "Latxaga" (2,07 km²) en "La Tejeria" (1,69 km²) zijn landbouwgebieden. Het "Oskotz Forestal" stroomgebied $\left(5,05 \mathrm{~km}^{2}\right.$ ) is een (semi-) natuurlijk stroomgebied. Gewassen in de landbouwgebieden zijn voornamelijk wintertarwe en rogge, terwijl in Oskotz voornamelijk grasland en bos aanwezig is. De geologie in Latxaga en La Tejeria wordt gekarakteriseerd door mergel met in La Tejeria een aantal zandsteenbanken. In Oskotz is een afwisseling tussen mergel en zandige kalksteen aanwezig in de ondergrond.

In hoofdstuk 2 heb ik netwerken (grafentheorie) gebruikt om de dynamiek van connectivity van opppervlakkige afstroming op hellingen in een sub-Mediterraan klimaat te karakteriseren door gebruik te maken van hoge-resolutie digitale terreinmodellen, sensoren voor oppervlakkige afstroming en netwerkanalyse. Resultaten lieten zien dat er significante verschillen zijn voor connectivity van 
oppervlakkige afstroming op landbouwgebieden en semi-natuurlijke struikgebieden. Er was een significant positieve relatie aan te tonen tussen neerslageigenschappen en connectivity, terwijl er een negatieve correlatie was tussen bodemvocht en connectivity. Deze laatste negatieve correlatie wordt hoogstwaarschijnlijk veroorzaakt door waterafstotendheid van de bodem in droge periodes, of door verslemping van de bodem. De combinatie van structurele netwerken en dynamische netwerken is een krachtig instrument gebleken voor het vaststellen van potentiele connectivity en daadwerkelijke connectivity, en het analyseren van connectivity van oppervlakkige afstroming.

In hoofdstuk 3 heb ik het functioneren van sediment connectivity tussen een helling en een geul bepaald en het transport van deze sedimenten in de geul bekeken. Om de sediment connectivity te bekijken heb ik gebruik gemaakt van zeldzame aardmetalen die sterk binden aan de bodem, zodat ze kunnen functioneren als tracer (Engels: volger). Deze tracers zijn opgebracht aan het begin van de herfst in 2014 en de de bemonstering vond plaats aan het begin van de zomer van 2015. Resultaten lieten zien dat er gedurende de meetperiode geen bodemdeeltjes van de helling naar de geul zijn getransporteerd. Analyses van neerslagdata toonden aan dat hoewel de totale neerslaghoeveelheid niet veel afweek van het gemiddelde, de neerslagintensiteit wel lager was. Door gebruik te maken van een machinaal leren algoritme genaamd Random Forest, kon ik laten zien dat de connectivity tussen de helling en de geul gedomineerd wordt door de mobilisatie van sedimenten gedurende grote (hoge intensiteit) regenbuien. Het gemobiliseerde sediment wordt voor het grootste gedeelte het stroomgebied uit getransporteerd, maar een ander gedeelte blijft aan het eind van de bui achter in de geul. Deze sedimenten worden later door kleinere buien het stroomgebied uit getransporteerd.

In hoofdstuk 4 heb ik laten zien dat bestaande data gebruikt kunnen worden om de bepalende factoren voor connectivity te determineren en te bekijken hoe deze factoren veranderen door de tijd. Data uit drie stroomgebieden in Navarra, Noord Spanje zijn gebruikt om de factoren voor hydrologische en sediment connectivity te bepalen. Deze factoren werden gebruikt als componenten in een niet-ruimtelijk model voor debiet en sedimentafvoer. Drie componenten werden onderscheiden: topografische, biologische en bodem. Veranderingen in de topografische component werden verondersteld gelijk gebleven te zijn en werden daarom constant gehouden. Veranderingen in de biologische component werden bepaald door het gebruiken van de Normalised Difference Vegetation Index (Engels: genormaliseerd verschil vegetatie index). Veranderingen in de bodem component werden bepaald door het gebruik van een antecedente neerslag index. Nash-Sutcliffe modelefficiëntie coëfficiënten waren tussen 0.49 en 0.62 voor de debietmodellen en tussen de 0.23 en 0.3 voor de sedimentuitvoer modellen. Ik heb aanbevolen om de modellen op kleinere schaal dan stroomgebiedsschaal te gebruiken om zo de ruimtelijke resolutie van de componenten te vergroten. 
In hoofdstuk 5 was het doel om een betere grip te krijgen op de implicaties van modelcalibratie op verschillende ruimtelijke schalen van simulaties van hydrologie en sedimentdynamiek in een agrarisch stroomgebied. Het LAPSUS-D model heb ik toegepast in het Latxaga stroomgebied. Het model is gekalibreerd en gevalideerd (4 jaar data: 2011-2015) met drie verschillende datasets op verschillende ruimtelijke schalen: helling, stroomgebied en helling+stroomgebied (gecombineerd). Het hellinggekalibreerde model liet voornamelijk oppervlakkige afstroming als gevolg van te lage infiltratie zien, het stroomgebied-gekalibreerde model liet voornamelijk oppervlakkige afstroming als gevolg van bodemverzadiging zien en het gecombineerd-gekalibreerde model liet oppervlakkige afstroming zien als gevolg van bodemverzadiging, maar alleen op de lagergelegen gedeeltes van de hellingen. Het gecombineerd-gekalibreerde model simuleerde de grote debietpieken van het gehele stroomgebied het beste, terwijl op de hellingschaal het helling-gekalibreerde model het beste presteerde. Voor sedimenten presteerde het helling-gekalibreerde model het beste met coëfficiënten van 0.618 voor kalibratie en 0.269 voor validatie. Daarnaast was dit het enige model dat de gemeten erosie en depositiepatronen juist wist weer te geven. De totale hoeveelheden erosie en depositie waren echter wel te hoog. De resultaten laten zien dat modelleurs er zich bewust van moeten zijn dat er vele problemen zijn met automatische kalibratie en overkalibratie wanneer er geen data van meerdere ruimtelijke niveaus worden meegenomen in de kalibratie. Het is daarom nodig om erosie en sedimenttransportmodellen te kalibreren en valideren met gemeten data zoals sediment tracing data en metingen van oppervlakkige afstroming op verschillende schalen en meetcampagnes daar specifiek voor in te richten.

De combinatie van hoofdstukken in dit proefschrift laat zien dat het concept connectivity een bruikbaar concept is voor een groot aantal studies, variërend van de hellingschaal tot stroomgebiedschaal. Met het gebruiken van het concept was ik in staat om de sedimentdynamiek in een sub-Mediterraan agrarisch stroomgebied te bepalen en te laten zien dat deze dynamiek anders is dan voorheen gedacht.

Afhankelijk van het doel van de studie, kunnen verschillende concepten van connectivity bruikbaar zijn. Verschillen in geologie en klimaat tussen stroomgebieden zorgen ervoor dat er grote verschillen zijn tussen de sedimentdynamiek. Daarom zou het misschien niet nodig zijn, of zelfs niet mogelijk zijn, om één enkel conceptueel kader te ontwikkelen voor connectivity. In plaats daarvan zou een verzameling van kaders voor verschillende omstandigheden moeten worden ontwikkeld. Deze kaders zouden echter altijd moeten helpen met het beslissen van welke metingen genomen moeten worden en welk type modellen en indices gebruikt zouden moeten worden voor een bepaalde omgeving.

Mijns inziens is connectivity zeker een bruikbaar concept om onze kennis over wateren sedimenttransportprocessen verder te ontwikkelen, hoewel het concept niet de uiteindelijke brug zal vormen die nodig is om het complete dynamische gedrag van processen binnen stroomgebieden over de gehele wereld te begrijpen. Het gat tussen de verschillende ruimtelijke en temporele schalen is te groot om met een enkel concept 
zoals connectivity te overbruggen. Echter, in de nabije toekomst zullen er vele studies gepubliceerd worden over connectivity die onze kennis over water- en sedimenttransportprocessen verder helpen. 


\section{Resumen}

La degradación del suelo es un grave problema en todo el mundo, en especial en las zonas agrícolas. Entre 1 y 6 mil millones de hectáreas de suelo de todo el mundo se ven afectadas por la degradación. Con una población mundial creciente, es necesaria una mayor producción de alimentos y, por tanto, una creciente superficie de tierras se dedica al uso agrícola. A su vez, esta transformación de terrenos trae consigo una mayor degradación del suelo. Algunas de las formas más comunes de degradación de tierras son la desertificación, la salinización y la erosión hídrica del suelo. Los efectos negativos de la erosión del suelo han sido documentados desde hace mucho tiempo. Desde principios del siglo XX, se ha intentado cuantificar el desplazamiento del suelo por el agua, así como medir y modelar la eficiencia de las estrategias de manejo del suelo.

Entre las consecuencias de problemas como el aumento de escala, la representación incorrecta de los procesos y la equifinalidad, destaca la dificultad de predecir adecuadamente las fuentes de sedimento y sus rutas y sumideros dentro de las cuencas hidrológicas. Estos problemas pueden dar lugar a la implementación de estrategias de manejo poco adecuadas. Así, con el fin de tratar estos procesos no lineales y también la carencia de una representación adecuada de las fuentes de agua y sedimentos, caminos y sumideros, se desarrolló el concepto de conectividad. Muchas han sido las definiciones de conectividad propuestas hasta el presente, aunque la más utilizada es la de la conectividad hidrológica de Pringle (2003): "La conectividad hidrológica es el transporte por el agua de la materia, la energía y los organismos, dentro o entre elementos del ciclo hidrológico".

Hoy día, dentro de las ciencias dedicadas al estudio de las cuencas hidrológicas, sigue siendo una prioridad el establecer una teoría unificada sobre lo que constituye la conectividad y sobre cómo debe medirse o inferirse. Asimismo, no está claro si la conectividad debe ser un resultado (output) o un elemento de entrada (input) de un modelo $y$, en caso de considerarse un input, si debe agregarse explícita o implícitamente. El objetivo principal de esta tesis fue, por tanto, evaluar y cuantificar la conectividad hidrológica y de sedimentos de una manera significativa, que pueda contribuir a una mayor comprensión de los procesos de hidrológicos y de transporte de sedimentos, así como de la dinámica de las cuencas hidrológicas como sistema.

El estudio se realizó en tres cuencas experimentales de Navarra, en el norte de España. Dos de las cuencas, 'Latxaga' y 'La Tejeria', son cuencas agrícolas, con una superficie de 2,07 y 1,69 km², respectivamente. La cuenca de 'Oskotz Forestal' es una cuenca seminatural, y ocupa $5,05 \mathrm{~km}^{2}$. El uso del suelo en las cuencas agrícolas está destinado principalmente al cultivo de trigo de invierno y cebada, mientras que en la cuenca de Oskotz predomina el pastizal y el bosque. Latxaga y La Tejeria tienen a las margas como principal material geológico subyacente, y dentro de La Tejeria también se encuentran areniscas. La geología de Oskotz se caracteriza por una alternancia de margas y calizas arenosas. 
En el capítulo 2 hice uso de redes (teoría de gráficos) para caracterizar y cuantificar la dinámica de la conectividad del flujo superficial en laderas en un ambiente submediterráneo húmedo utilizando una combinación de modelos de terreno digital de alta resolución, sensores de flujo superficial y un enfoque de red. Los resultados mostraron la existencia de diferencias significativas entre la conectividad del flujo en áreas cultivadas y la de áreas arbustivas seminaturales. Se obtuvieron correlaciones positivas significativas entre la conectividad y las características de la precipitación. Por otro lado, se encontraron correlaciones significativas negativas entre conectividad y humedad del suelo, muy probablemente debidas a repelencia y/o a la presencia de costra superficial. La combinación de redes estructurales y redes dinámicas para determinar la conectividad potencial y la conectividad real demostró ser una herramienta poderosa para analizar la conectividad de flujo superficial.

En el capítulo 3 determiné el funcionamiento de la conectividad entre la ladera y el canal, así como la continuidad del transporte de estos sedimentos en el canal. Para determinar este funcionamiento, obtuve datos del transporte de sedimentos desde las laderas hasta los canales, al mismo tiempo que examinaba los factores que influyen en la exportación de sedimentos de la cuenca. Para medir la conectividad ladera-canal de los sedimentos, se aplicaron trazadores de óxido de "tierras raras" (REO) en una ladera en la cuenca de Latxaga antes del comienzo del invierno de 2014-2015. Los resultados mostraron que durante el invierno no se transportaron sedimentos desde la ladera hasta el canal. El análisis de los datos de precipitación demostró que, aunque las cantidades totales de precipitación no difirieron mucho de la media, las intensidades de precipitación fueron bajas. Usando "Random Forest" (RF), un método de aprendizaje automático, comprobé que la conectividad ladera-canal en Latxaga está controlada por la movilización de sedimentos durante eventos de lluvia importantes y de alta intensidad. Los sedimentos son en gran parte exportados durante este tipo de eventos. Los eventos importantes también dejan grandes cantidades de sedimentos en el canal y en su entorno, los cuales son eliminados gradualmente por pequeños eventos.

En el capítulo 4 expliqué cómo los datos existentes pueden utilizarse para evaluar los factores que controlan la conectividad y cómo estos factores cambian con el tiempo. Se utilizaron datos de tres cuencas de Navarra, en el norte de España, para evaluar factores que influyen en la conectividad hidrológica y del sedimento. Estos factores se utilizaron como componentes en un modelo lineal agregado, para la descarga por un lado y para la exportación de sedimentos suspendidos por otro. Se distinguieron tres componentes de conectividad: topográficos, biológicos y edáficos. Los cambios en el componente topográfico durante los períodos estudiados se consideraron relativamente pequeños, de modo que dicho componente se mantuvo constante. Los cambios en el componente biológico se determinaron usando el Índice de Diferencia de Vegetación Normalizada. Finalmente, los cambios en el componente del suelo se evaluaron utilizando un Índice de Precipitación Antecedente. Los coeficientes de eficiencia del modelo (Nash-Sutcliffe) se encontraban entre 0,49 y 0,62 para los modelos de descarga y entre 0,23 y 0,3 para los modelos de exportación de sedimentos. Recomendé aplicar el modelo a escalas espaciales más pequeñas que la 
escala de cuenca con el fin de minimizar el efecto de la agregación de la variabilidad espacial de los componentes.

En el capítulo 5, el objetivo fue mejorar nuestro conocimiento de las implicaciones de la calibración de modelos en diferentes escalas espaciales sobre la simulación de la hidrología y la dinámica de sedimentos en una cuenca agrícola. Utilicé el modelo LAPSUS-D en la cuenca de Latxaga. El modelo fue calibrado y validado (4 años: 2011-2015) utilizando tres bases de datos a diferentes escalas espaciales: ladera, cuenca y de bases de datos combinadas (modelo combinado-calibrado). El modelo calibrado para la ladera mostró principalmente una escorrentía generada por exceso de lluvia; por otro lado, el modelo calibrado a nivel de cuenca mostró principalmente una escorrentía generada por saturación en las zonas situadas entre las partes inferiores y medias de las laderas. En referencia a la hidrología, el modelo combinadocalibrado fue el que mejor simuló los mayores picos de caudal, mientras que a escala de ladera, el modelo calibrado para ladera fue el que mostró los mejores resultados. Este último modelo fue el que dio lugar a las mayores eficiencias para sedimentos en calibración (0.618) y validación (0.269). El modelo calibrado para laderas fue el único modelo que mostró erosión por cárcavas sobre un Modelo Digital de Elevaciones (DEM) de alta resolución, y mostró también la dinámica del sedimento en el canal. Sin embargo, las cantidades simuladas absolutas de erosión y deposición dentro de la cuenca resultaron demasiado altas. A partir de estos resultados puede decirse que es conveniente que los modeladores estén al corriente de los problemas asociados a la calibración automática, a la sobre-calibración y a la no incorporación de datos, medidos a múltiples escalas espaciales. Recomendamos encarecidamente la incorporación de datos de escorrentía y de trazadores de sedimento a múltiples escalas siempre que sea posible y, asimismo, la realización de campañas de medición específicas con este fin, para obtener una visión más completa de la conectividad hidrológica y del sedimento dentro de una cuenca.

La combinación de los capítulos de esta tesis demuestra que el concepto de conectividad es útil en una amplia gama de estudios, desde la escala de ladera hasta la escala de cuenca. Utilizando el concepto de conectividad, pude determinar la dinámica de los sedimentos de una cuenca mediterránea húmeda y demostrar que este comportamiento es diferente de lo que se pensaba anteriormente.

Dependiendo del objetivo del estudio, numerosos conceptos de conectividad son de utilidad. La diversidad de entornos geológicos y climáticos genera grandes diferencias en la dinámica (del sedimento) en las cuencas. Por tanto, no sería necesario, ni siquiera posible, buscar un marco conceptual único y unificador para la conectividad. Sin embargo, sería ventajoso desarrollar una compilación de marcos para los diferentes contextos. No obstante, estos marcos deben tener siempre como objetivo ayudar a entender qué medidas deben ser tomadas y qué tipo de modelos e índices han de usarse para ese entorno en particular.

Opino honestamente que la conectividad es sin duda un concepto útil para incrementar nuestro conocimiento sobre los procesos de transporte de agua y sedimentos. Sin 
embargo, también se requiere de una cuidadosa reflexión, ya que este concepto en particular no necesariamente proporcionará la explicación concluyente del comportamiento dinámico en las cuencas hidrográficas de todo el mundo. La brecha entre las diferentes escalas espaciales y temporales es demasiado compleja como para ser zanjada con un solo concepto como la conectividad. Sin embargo, los numerosos estudios sobre conectividad que se publicarán en un futuro próximo podrán contribuir a mejorar el conocimiento de los procesos de transporte de agua y sedimentos. 


\section{Dankwoord / Agradecimientos / Acknowledgements}

Al met al ben ik meer dan vijf jaar bezig geweest met dit proefschrift en gedurende deze tijd hebben veel mensen mij direct en indirect geholpen bij het bereiken van deze mijlpaal in mijn leven en deze mensen wil ik natuurlijk graag bedanken!

Allereerst wil ik graag mijn promotoren en co-promotoren bedanken. Coen bedankt dat je na mijn stage bij Alterra genoeg vertrouwen in me had om me als kandidaat voor te stellen aan Saskia. En Saskia bedankt dat je dat advies van Coen destijds aan hebt genomen en daarmee ook mij had aangenomen! Je wist me vaak terug te zetten op het juiste pad, wanneer ik weer eens afgedwaald was in een jungle van nog meer nieuwe dingen proberen. Daarnaast het stellen van de af en toe zo nodige harde deadlines was zo af en toe ook zeker nuttig. Arnaud, ook al ben je pas later aangesloten, je bent erg belangrijk geweest bij alle discussies, vooral bij al het modelleren. En je hebt me zelfs nog geholpen om sensoren de grond in te hameren op een warme oktoberdag. Sjoerd bedankt dat je de rol van promotor op je wilde nemen, zodat mijn verdediging gewoon door kon gaan.

Ik heb veel gehad aan alle afleiding van alle vrienden in Utrecht en in Gramsbergen. Er zijn te veel namen om allemaal op te noemen dus doen we het maar in groepjes: de FG boys voor de avondjes Derrick/Stathe, de mannen van het Sciencemedia zaalvoetbalteam voor de eerste twee helften van matige kwaliteit, maar derde helften van wereldklasse, alle dames en heren uit Gramsbergen voor het ouwehoeren en de weekendjes weg.

Dan mijn paranimfen; Tim, aan het begin van mijn promotie waren het voornamelijk de filmavonden op dinsdag en de biertjes op de trap voor het huis en later werden het weekendjes naar Londen. Dat alles zorgde in ieder geval voor genoeg vertier! Niels bedankt voor alle leuke momenten tijdens en buiten het werk om. We zijn 3 keer samen naar Pamplona gegaan, in principe om DEMs te maken, maar die pintxos en wijntjes waren een leuke bijkomstigheid!

Pap \& mam bedankt dat jullie me altijd ondersteunden en me helemaal vrij lieten in mijn keuzes. Zeker toen ik bedacht had dat ik na een jaar Industrieel Ontwerpen toch Aardwetenschappen wilde studeren, anders was ik nu niet hier geweest. Irmi, grote zus, vele momenten tijdens mijn promoveren wilde ik wel dat ik wat meer georganiseerd was zoals jij. Ik kijk er erg naar uit om mijn toekomstige neefje/nichtje van alles bij te brengen over de aarde, maar natuurlijk over bodemerosie het meest!

Thanks to all the people from SLM and the Atlas-gang, for all the nice work-distracting lunches and coffee breaks! I'd especially like to thank my (former) office mates Ate, Joep, Hong-Ming, Esperanza, YueLing and Ricardo for the necessary serious and less serious discussions. Same goes for the lab meeting group; receiving feedback and having to give feedback every week stimulated me a lot this last year. Thanks also to Piet, Hennie and Harm for helping with many technical and lab-related issues! For all financial and logistical matters, I'd like to thank Marnella, Esther, Anita and Siawash. 
Big thanks also to all the people that are involved in the COST Action Connecteur. We had a lot of very interesting discussions during our meetings and I do think we came a long way in those four years. I definitely learned a lot which was partly applied in this thesis.

There were other people involved with some of the articles in this thesis who I'd like to thank. Thanks Manuel for the discussions and help, especially at the beginning of my $\mathrm{PhD}$. Tobias, I went to visit you twice in Eichstätt and I had a great time there trying to figure out all the issues we ran into while trying to create the networks on the high-res DSMs, so thanks for a great experience and collaboration Another big thanks to Joao for helping me with the modelling part and for sharing beers all over Europe.

Micaela thanks a lot for offering a very nice 3-week holiday distraction from the PhD by getting married in Argentina and thanks a lot for the help with the cover design!

Gracias a toda la gente de España que me ha ayudado en esta etapa tan importante de mi vida. Primero a mi familia de España: José Luis, Isabel y Pablo, siempre me hacéis sentir como en casa cuando estoy en Burgos o en Somo, La comida de Isabel y los billares con Pablo y José me han dado mucha energía para sobrellevar el doctorado.

En Pamplona, gracias a toda la gente de la Universidad que también me ha ayudado mucho. He conocido a mucha gente en los descansos y las comidas, y allí aprendí un mogollón de castellano y un pelín de euskera. Sobre todo, gracias a Alex, Paul, Youssef y Javi, me habéis ayudado mucho desde echarme una mano en el trabajo de campo hasta enseñarme todos los rincones de Pamplona donde comer los mejores pintxos y la mejor croqueta. Javier y Rafa, vosotros habéis sido una ayuda indispensable, muchas horas hablando sobre conectividad, incluso más sobre los manuscritos de los artículos y también habéis estado ahí para cosas logísticas como comunicarme con agricultores cuando yo no sabía ni papa de Español.

Y por último, pero no menos importante: Lieve Laura, thanks for being there for me during my journey towards this PhD. It made the way a whole lot more comfortable knowing that you were there to help me out with any problem or stressful moment I had. I am happy to have had you as a support and even happier to keep on having it in the many days that we still have ahead of us together. 


\section{Publications}

\section{Published peer reviewed articles}

Masselink, R.J.H., Heckmann, T., Temme, A.J.A.M., Anders, N.S., Gooren, H.P.A., Keesstra, S.D., 2016. A network theory approach for a better understanding of overland flow connectivity: Networks for a better understanding of overland flow connectivity. Hydrol. Process. doi:10.1002/hyp.10993

Masselink, R.J.H., Keesstra, S.D., Temme, A.J.A.M., Seeger, M., Giménez, R., Casalí, J., 2016. Modelling Discharge and Sediment Yield at Catchment Scale Using Connectivity Components. Land Degrad. Dev. 27, 933-945. doi:10.1002/ldr.2512

Masselink R.J.H, Temme AJAM, Giménez R, Casalí J, Keesstra SD. 2017. Assessing hillslope-channel connectivity in an agricultural catchment using rare-earth oxide tracers and random forests models. Cuadernos de Investigación Geográfica

Wickama, J., Masselink, R.J.H, Sterk, G., 2015. The effectiveness of soil conservation measures at a landscape scale in the West Usambara highlands, Tanzania. Geoderma 241-242, 168-179. doi:10.1016/j.geoderma.2014.11.020

Baartman, J.E.M., Masselink, R.J.H., Keesstra, S.D., Temme, A.J.A.M., 2013. Linking landscape morphological complexity and sediment connectivity. Earth Surf. Process. Landf. 38, 1457-1471. doi:10.1002/esp.343

Anders, N.S., Masselink, R.J.H., Keesstra, S.D., Suomalainen, J., 2013. High-Res Digital Surface Modeling using Fixed-Wing UAV-based Photogrammetry, in: Proceedings of Geomorphometry 2013. Presented at the Geomorphometry 2013, Nanjing, China, pp. 16-20

\section{Selection of first author conference abstracts}

Masselink, R.J.H., Heckmann, T., Temme, A.J.A.M, Anders, N.S., Keesstra, S.D. 2016. A network theory approach for a better understanding of overland flow connectivity. Geophysical Research Abstracts Vol. 18, EGU2016-244, 2016 EGU General Assembly 2016

Masselink, R.J.H., Keesstra, S.D., Temme, A.J.A.M., Giménez, R., Casalí, J., Seeger, M. Using a new conceptual framework to assess sediment connectivity. Geophysical Research Abstracts Vol. 17, EGU2015-15824, 2015 EGU General Assembly 2015

Masselink, R.J.H., Anders, N.S., Keesstra, S.D, Seeger, M., 2014. Mapping temporal changes in connectivity using high-resolution aerial data and object based image analysis. In EGU General Assembly Conference Abstracts, Vol. 16, EGU2014-14479

Masselink, R.J.H., Keesstra, S.D., Seeger, M., 2014. Assessment of catchment scale connectivity in different catchments using measured suspended sediment output. In EGU General Assembly Conference Abstracts, Vol. 16, EGU2014-14637

Masselink, R.J.H., Keesstra, S.D., Gimenez, R., Casali, J., Seeger, M., 2013. Determining catchment-scale connectivity using hysteresis analysis of discharge and suspended sediment. In: Proceedings of the 8th IAG International Conference on Geomorphology, Paris, France, 27 - 31 august, 2013

Masselink, R.J.H., Keesstra, S.D., Seeger, K.M., 2013. Mapping connectivity for modelling catchment scale runoff and sediment yield. EGU2013-3233, 2013 EGU General Assembly 


\section{About the author}

Rens Jan Hein Masselink was born on 14 March 1986 in Hardenberg and grew up in Gramsbergen, The Netherlands. He attended high school at the Nieuwe Veste in Coevorden. He finished a BSc and MSc "Physical Geography" at the University of Utrecht in 2008 and 2011, respectively. Especially during his masters, he developed an interest for earth observation and natural hazards like land degradation. He completed an MSc thesis on automatic

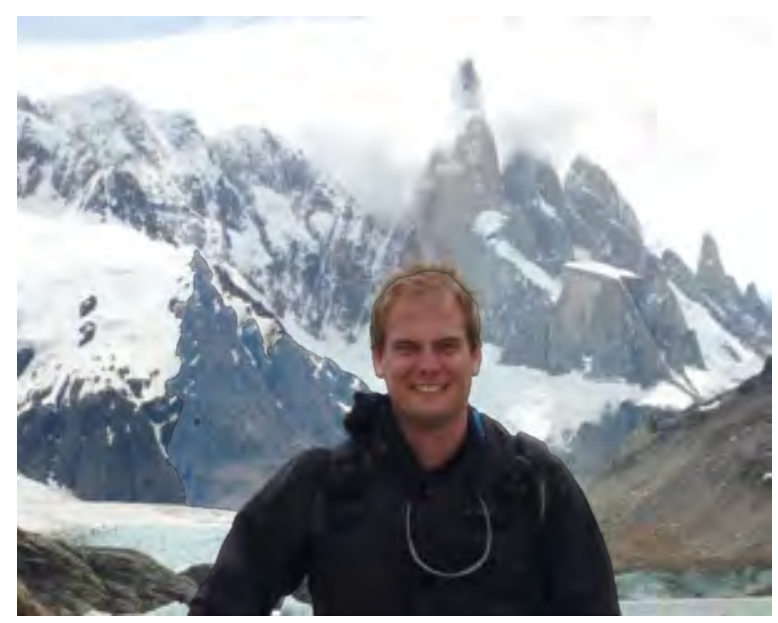
classification of soil and water conservation measures from satellite imagery and erosion risk modelling in the Usambara highlands in Tanzania. He worked for some months at Hydrologic Research, a consultancy/research company involved in projects involving hydrological modelling.

Rens started his PhD at the Soil Physics and Land Management Group at the University of Wageningen in 2012 and did all of the fieldwork for his PhD in Navarre, Spain. He worked together with academics from the Public University of Navarre and the Universities of Trier and Eichstätt-Ingolstadt in Germany. Next to his PhD research he was working as a grant holder administrator for an EU funded COST action called "Connecting European Connectivity Research" in which he also actively participated. These collaborations are still ongoing at the time of the publication of this dissertation. In 2017, he started a Postdoctoral project which is aimed at assessing natural resources through the use of Unmanned Aerial Vehicles (drones). 


\section{SENSE}

Netherlonds Research School for the

Sacia-Economic and Natural Sciences of the Environment

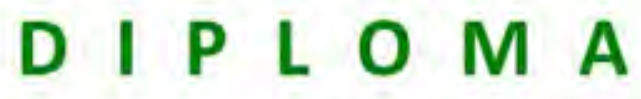

For specialised PhD training

The Netherlands Research School for the Socio-Economic and Natural Sciences of the Environment

(SENSE) declares that

\section{Rens Jan Hein Masselink}

born on 14 March 1986 in Hardenberg, The Netherlands

has successfully fulfilled all requirements of the Educational Programme of SENSE.

Wageningen, 7 September 2017

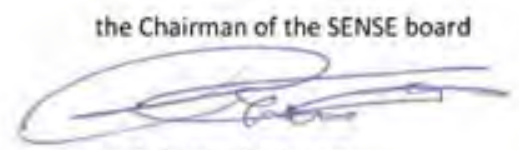

Prof. dr. Huub Rijnaarts the SENSE Director of Education

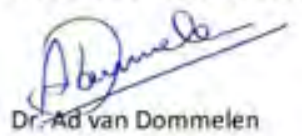

The SENSE Firsearch School has bern occredited by the Aloyol Netherlands Acoudemy of Ants onef Scienews (XNAW)

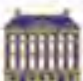

KO N I N K L I J K E N E D E R L A N D S E

A K A D E M I E V A N W E T E N S C H A P P E N 


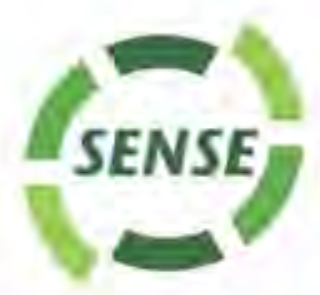

The SENSE Research School declares that Mr Rens Masselink has sutcessfilly fulfilied all requirements of the Equcabional PhD Programme of SENSE with a work load of $39 \mathrm{EC}$, inclueing the following activitiac:

\section{SENSE PhDC Courses}

a) Environmental research in context (2012)

a. Research in context activity, 'Creating an overview of communicating susgestions for presenting 'Science in the Classroom', as well as applving these insights for a press release and a general audience publication' (2017)

\section{Other PhD and Advanced MSc Courses}

- Training Afstudeervak organiseren en begeleiden, Wageningen Urviversity (2013)

- (ecturing. Wageningen university (2013)

- Workshop Presentation skills, Wageringen University (2013)

- Geostatistics, Wageningen University (2015)

- Techniques for writing and presenting a scientific paper, Wageningen University (2015)

- Writing grant proposals, Wogeningen University (2016)

\section{External training at o forelen research institute}

1. Couperation with expert in graph theory, Eichstart University, Gemany (2015)

\section{Management and Didactic Skills Training}

a. Supervising three MSc student with thesis entitled 'Explaining hydrolokical connectivity using sol moisture characteristics on hellslope lever' (2014), "Otject-Based Classification of Gullies using UAV-Derived Digital Elevation Models (2015) and 'Using semi-automated hysteresis analyses for determining catchment cannectivity (2017)

a Teaching in the M5c courses 'Fundamentals of Land Management' (2013-2016). 'Soil erosian' (2016) and 'Soil ercsion Modelling' (2017)

a Assisting in practical training of the MSc courses 'Geohydrology' (2012-2016) and 'Fundamentats of Land Management' (2013, 2016)

Q. Co-organising workshop 'Connecting European Connectivity Research' 24-26 August 2014; Wageningen

\section{Oral Presentations}

- Assessment of catchment scale connectivity using measured suspended sediment output. European Geosciences Union General Assembly 2014, 27 April - 2 May 2014, Vienna, Austria

- A network theory approoch for a better understonding of overland flow connectivity. European Geosciences Union General Assembly 2016, 17-22 April 2016, Vienna, Austria

- Scale matters. contrasting hillslope and catchment scale calibration results for sediment fluxes using the LAPSUS-O model. Working Group meeting Cost Action 'Connecting European Connectivity Research (Connecteur)',21-23 September 2016, Prague, Crech Republic

SENSE Coordinator PhD Education

Dr. ing. Monique Gulickx 

This project was financially supported by the Dutch Organisation for Scientific Research (NWO) grant number 822.01.004, by the Spanish Ministry of Science and Innovation, project number CGL2011-24336 and by COST Action ES1306: "Connecting European Connectivity Research".

Cover photo: Rens Masselink

Cover Design: Micaela Tettamanti 